\title{
Update and challenges in organo-mediated polymerization reactions
}

Winnie Nzahou Ottou, ${ }^{[a]}$ Haritz Sardon, ${ }^{[b]}$ David Mecerreyes, ${ }^{[b]}$ Joan Vignolle, ${ }^{[a]}$ and Daniel Taton*[a]

[a] CNRS/Univ. Bordeaux, LCPO, UMR 5629, 16 av. Pey-Berland, F-33607 Pessac Cedex (France)

[b] POLYMAT Institute for Polymer Materials, University of the Basque Country UPV/EHU, Joxe Mari Korta Center, Avda. Tolosa 72, 20018 Donostia-San Sebastian (Spain)

${ }^{*}$ Corresponding author. Tel.: +33 5400084 82; fax: +33 540008487 . E-mail address: taton@enscbp.fr (D. Taton).

Keywords: Organic catalyst - Ring-opening polymerization - Step-growth polymerization - Group transfer polymerization - Acid catalysis - Basic catalysis • Bifunctional activation • Direct initiation •

\section{Table of contents}

Introduction. 3

1. Scope of organic activators, monomer candidates and general polymerization mechanisms. . .4

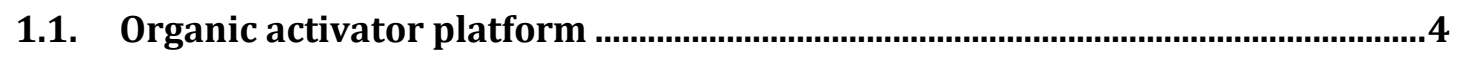

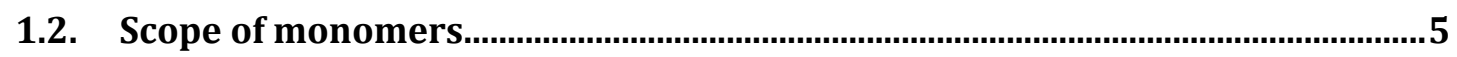

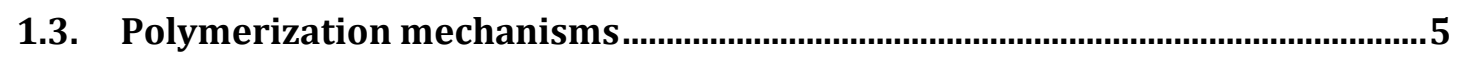

2. Polymerization catalyzed by Brønsted and Lewis acids .......................................... 11

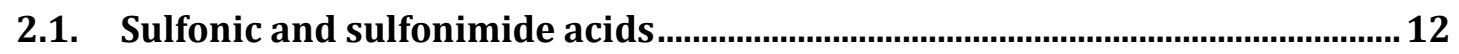

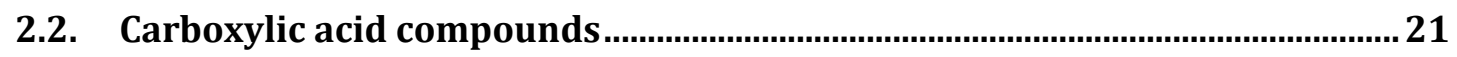

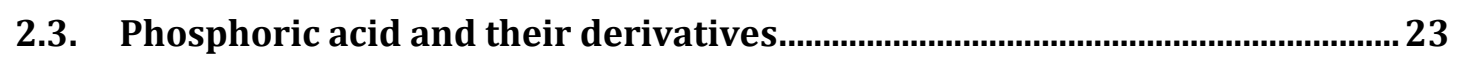

3. Polymerization catalyzed by phosphorous-containing Brønsted and Lewis bases:

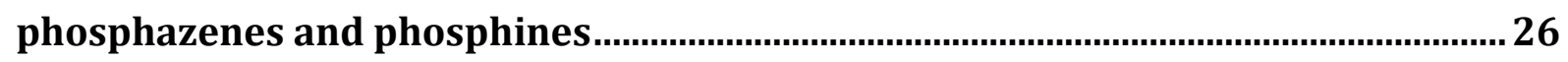

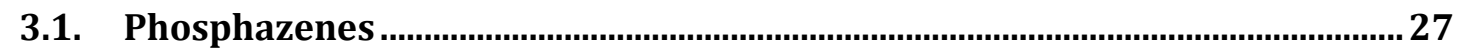


4. Polymerization catalyzed by free and latent $N$-heterocyclic carbenes (NHCs).... 34

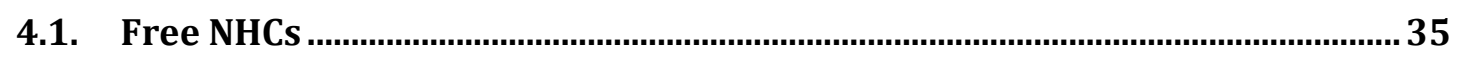

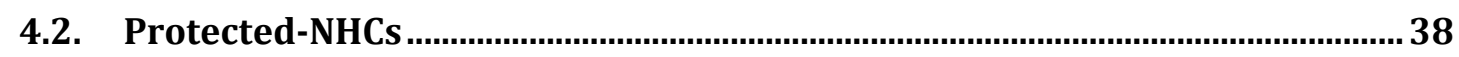

5. Polymerization catalyzed by nitrogen-containing Brønsted and Lewis bases: alkyl amines, amidines and guanidines...................................................................... 43

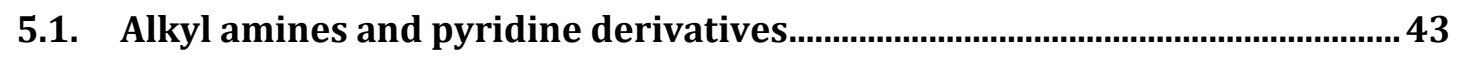

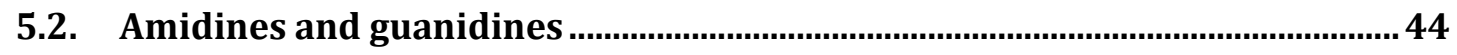

6. Mono- or bicomponent dual catalytic systems ....................................................... 54

7. Polymerizations directly induced by organic initiators ..........................................64

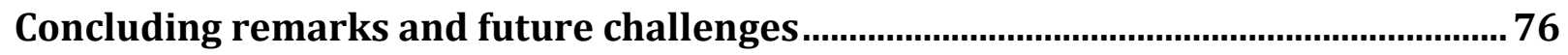

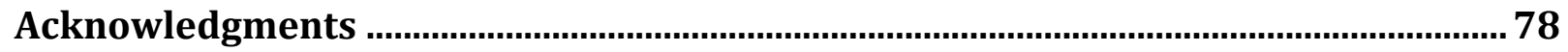

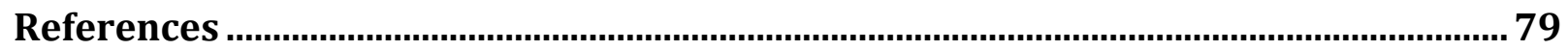

\begin{abstract}
Organocatalysis has become a very powerful tool for precision macromolecular chemistry, as judged by the number of articles published in this field in the past decade. A variety of small organic molecules, including Brønsted/Lewis bases and acids, based on amines, phosphines or carbenes, but also on bi-component systems, have been employed as a means to catalyze the polymerization of miscellaneous monomers. Not only can organocatalysts be employed to promote the ring-opening polymerization of various heterocyclics (e.g. lactones, lactide, cyclic carbonates, epoxides, lactams, cyclocarbosiloxanes), but some of them also allow activating vinylic monomers such as (meth)acrylics, or triggering the step-growth polymerization of monomers such as diisocyanates and diols for polyurethane synthesis. The least toxicity of organocatalysts in comparison to their metallic counterparts is also driving their development in some sensitive applications, such as biomedical or microelectronics. Overall, organocatalysts display specific monomer activation modes, thereby providing a unique opportunity to control the polymerization of various functional monomers, under mild conditions. This review article focuses on advances of the past 4 years (> 150 publications) in polymerization reactions utilizing small organic molecules either as direct initiators or as true catalysts, with a special emphasis on monomer activation modes, as well as polymerization mechanism aspects.
\end{abstract}




\section{Introduction}

Organocatalysis, i.e. the use of small organic molecules to catalyze chemical reactions, has gained increased attention over the past 15 years, with an emphasis on asymmetric variants, allowing a variety of biologically active compounds to be accessed.[1] For a long time, highly stereoselective transformations have been mainly achieved using enzymes and transition metal catalysts. However, organocatalysis has become the third branch of catalysis, providing various advantages, including: (i) environmentally more friendly and inherently lower toxicity of organic small molecules, (ii) higher availability of organic reagents, (iii) lower sensitivity toward oxygen and moisture and (iv) large chiral pool.[1]

The scope of organocatalytic systems, and their role in various elementary reactions of molecular chemistry have been discussed in detailed reviews.[2-5] They have also been introduced in macromolecular synthesis, where organic reagents can promote polymerization reactions, either as catalysts or as direct initiators, producing polymeric materials exempted of any metallic residues. Related metal-free polymers are thus expected to be employed in highvalue and sensitive domains, such as biomedical and personal beauty care applications, microelectronic devices or food packaging.

Several classes of organic activators (catalysts or initiators), including Brønsted/Lewis acids or bases, and mono- or bicomponent bifunctional catalytic systems have been utilized, not only for step-growth and chain-growth polymerizations, but also for depolymerization reactions in a context of recycling polymeric materials.

In recent years, organic activators have also been applied in biorefining processes for biomass conversion and upgrading into sustainable chemicals, materials, and biofuels as alternatives to petroleum-based compounds.[6] Metal-free polymerizations of bio-based monomers offer a new strategy for the development of high-performance bioplastics with enhanced thermal stability and solvent resistance.

A general review on organo-catalyzed polymerizations has been published in 2010 by Hedrick, Waymouth et al.,[7] and related specific topics, such as, ring-opening,[8, 9] grouptransfer,[10] anionic,[11] zwitterionic[12] polymerizations, and polymerizations induced by $\mathrm{H}$ bond catalysts[13] have also been reported. Our group has also reviewed the general field of organo-catalyzed polymerizations at the end of 2011[14] and, more specifically, in 2013, that regarding the use of $\mathrm{N}$-heterocyclic carbenes (NHCs) as organic catalysts for metal-free polymer synthesis.[15] While preparing this manuscript, Naumann and Dove reported recent trends of polymerizations utilizing NHCs either as organic catalysts or as direct initiators.[16]

From the end of 2011 to early 2015 , roughly 150 papers describing the use of organic catalysts in polymerization reactions have appeared (Figure 1), demonstrating the ever increasing interest of organocatalysis as a new tool for macromolecular engineering. 


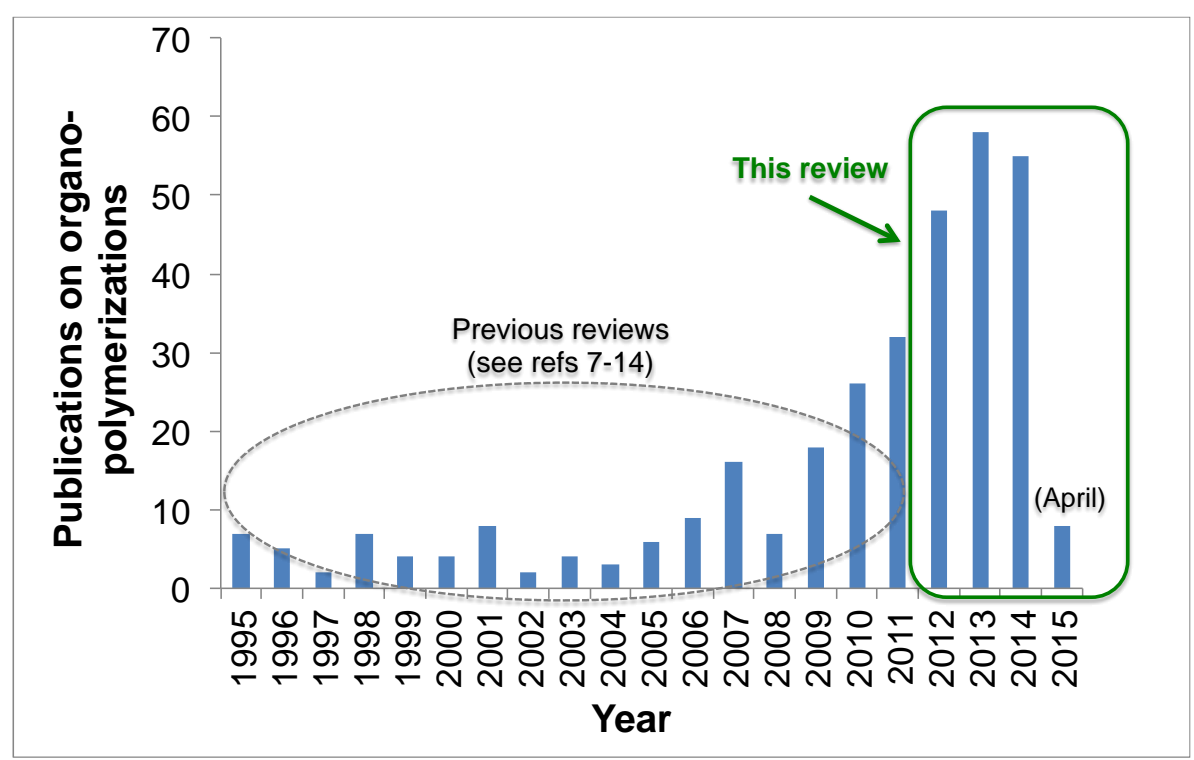

Figure 1. Evolution of publications on the topic of metal-free polymerizations since 1995. Data were obtained by a search in SciFinder and Web of science using the keywords "organocatalyzed polymerization", "metal-free polymerization" and derivatives (April 2015). Fig updated

The present review focuses on these recent developments and on applications of related metal-free polymers. The structure of main organic activators (catalysts or initiators) employed in polymerization reactions is first presented, and classified according to the nature of their functional group (i.e. acid or basic compounds, or activators featuring hydrogen-bonds with a donating or an accepting capability). Main monomer substrates and general polymerizations mechanisms are also briefly described. A focus on each category of activators is then given in the context of organo-catalyzed polymerizations although, in some cases, the catalyst is not employed in substoichiometric amounts relative to the initiator. The last section of this review deals with organo-initiated (non-catalyzed) polymerizations utilizing specific organic initiators, i.e. in absence of any other co-activator.

\section{Scope of organic activators, monomer candidates and general polymerization mechanisms}

\subsection{Organic activator platform}

A variety of metal-free compounds have been employed as catalysts or initiators in polymerization reactions (Figure 2), and most of them have been discussed in previous reviews.[7, 8, 14] They include Brønsted acids (e.g. sulfonic, phosphoric and carboxylic derivatives), Lewis acid (e.g. trimethylsilyltrifluoromethanesulfonimide: $\mathrm{Me}_{3} \mathrm{SiNTf}_{2}$ ), Brønsted or Lewis bases (e.g. phosphazenes, $\mathrm{N}$-heterocyclic carbenes, amines, phosphines, amidines or guanidines). Combination of antagonist properties, within the same molecule or not, leads 
to bifunctional ambiphilic systems. Generally, such a dual activation is based on hydrogenbond interactions (e.g. thioureas and phenols derivatives).[13]

\subsection{Scope of monomers}

Figure 3 illustrates representative monomer families recently polymerized using organic activators. Cyclic esters (lactides and lactones) have been the most studied in the context of organo-catalyzed ring-opening polymerization (OROP). $[7,8,14]$ This is not only due to the significant importance of corresponding biodegradable, non-toxic and in vivo bioresorbable polyesters, but also to the relative easiness for polymerizing these polar cyclic monomers. Cyclic carbonates have received a special attention, because related polycarbonates exhibit a lower degradation rate in water and allow for an easier introduction of reactive functionalities compared to other aliphatic polyesters, such as polylactide (PLA) or polylactones.[9] Other cyclic monomers, such as cyclic ethers, cyclosiloxanes, $\mathrm{N}$-carboxyanhydrides (NCAs), $\mathrm{O}$ carboxyanhydrides (OCAs) and phosphoesters, have also been reported to undergo metalfree ROP.

Besides ROP, (meth)acrylic monomers have been subjected to the group transfer polymerization (GTP), in the presence of silyl ketene acetal (SKA) initiators;[10] they were also evidenced to undergo a direct 1,4-conjugate addition polymerization, using a specific nucleophile (e.g. NHC).[17-19] A few examples of step-growth polymerizations leading to polyurethanes or polyaldols have also been reported.[20-22]

\subsection{Polymerization mechanisms}

The mechanism involved in organo-catalyzed polymerization reactions obviously depends on both the nature of the catalyst/chain starter and the monomer polymerizability. Polymerization can occur through an activated monomer mechanism (AMM), following either an electrophilic or a nucleophilic pathway. Polymerization operating by activation of a purposely-added initiator (or via activation of the polymer chain-ends) refers to as the activated chain-end mechanism (ACEM). A cooperative dual activation of both the monomer and the initiator can also take place with specific mono- or bicomponent hetero-bifunctional organic catalysts.

\subsubsection{Activated monomer mechanisms (AMM)}

Electrophilic reagents, such as Brønsted organic acids ( $E$ in Scheme 1), alkylating or acylating agents, can activate the monomer, e.g. heterocycles such as lactide (LA) or ethylene oxide (EO), by interaction with the heteroatom, e.g. the oxygen atom of the carbonyl group $(C=O)$ of cyclic esters and carbonates, the phosphoryl group $(P=O)$ of phosphoesters, or the oxygen atom of epoxides. 


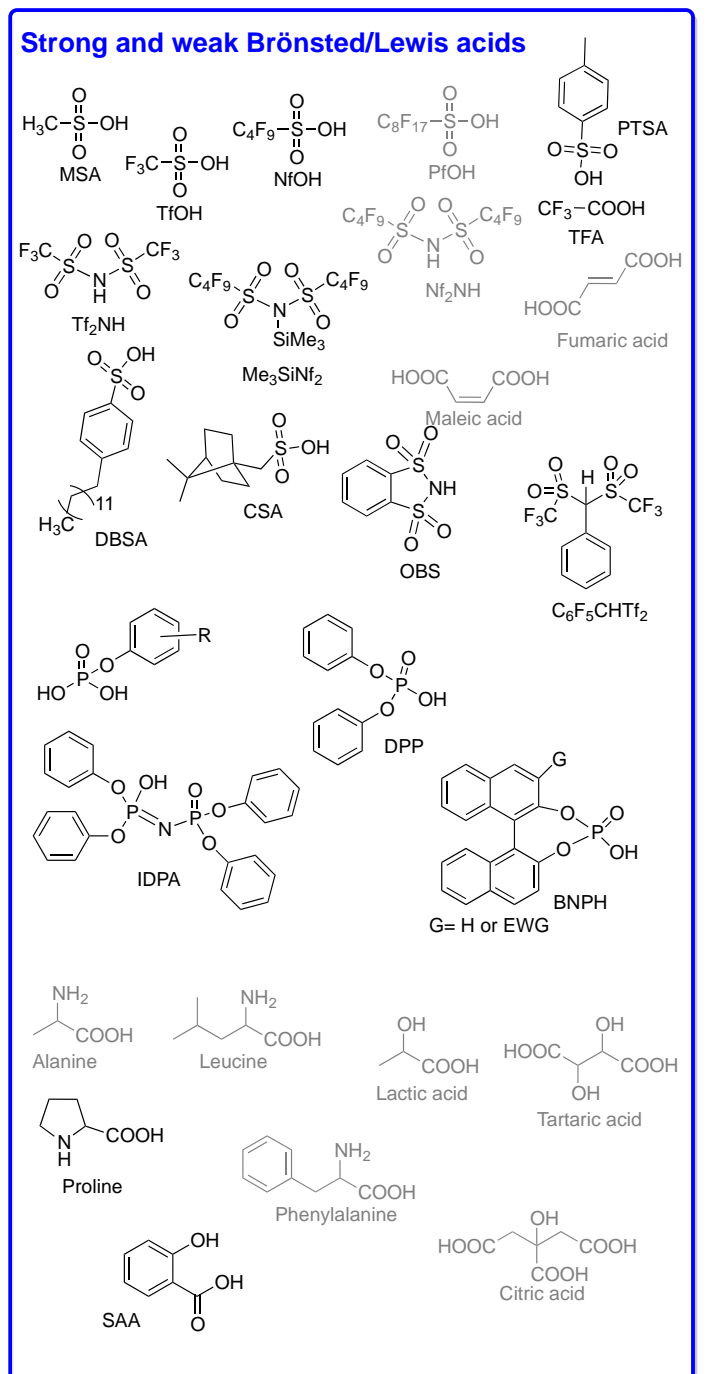

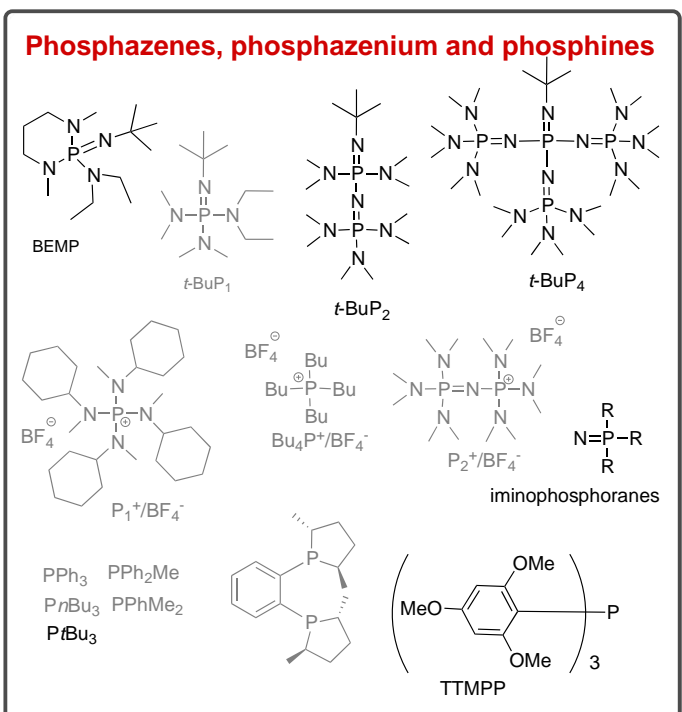

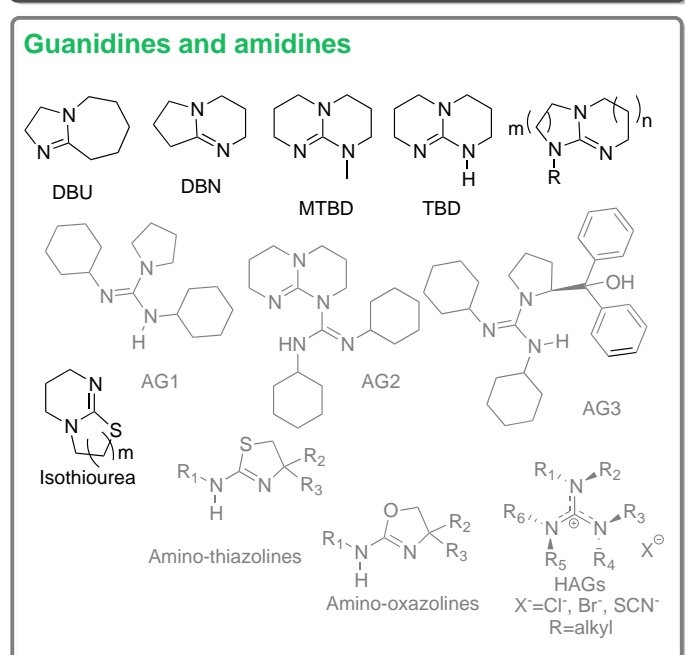

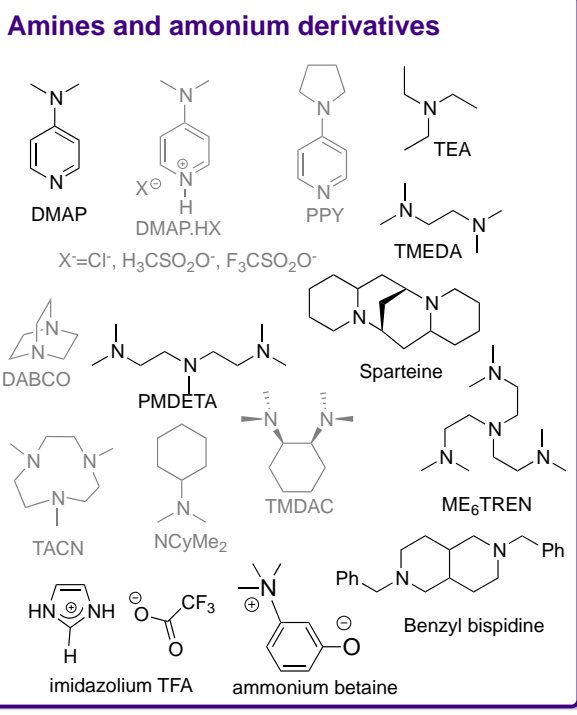

Thiourea-amino and phenolic derivatives

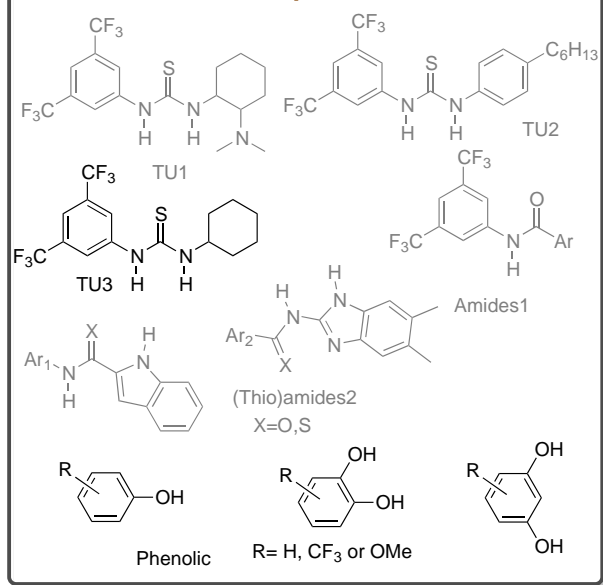

$\mathrm{N}$-Heterocyclic carbenes

Imidazol-type

$$
\mathrm{R}_{1}-\overbrace{}^{-N} \gamma^{N_{-} R_{2}}
$$

$\mathrm{NHC1} \quad \mathrm{R}_{1}=\mathrm{R}_{2}=\mathrm{Me}$

$N H C 2 \quad R_{1}=R_{2}=\mathrm{PPr}$

$\begin{array}{ll}\mathrm{NHC3} & \mathrm{R}_{1}=\mathrm{R}_{2}= \pm \mathrm{Bu} \\ \mathrm{NHC4} & \mathrm{R}_{1}=\mathrm{R}_{2}=\mathrm{C}_{12} \mathrm{H}_{25} \text { (Doc) }\end{array}$

$\begin{array}{ll}\mathrm{NHC5} & \mathrm{R}_{1}=\mathrm{R}_{2}=\mathrm{C} \\ \mathrm{NHC6} & \mathrm{R}_{1}=\mathrm{R}_{2}=\mathrm{A}\end{array}$

NHC7 $\mathrm{R}_{1}=\mathrm{R}_{2}=2,4,6-\mathrm{Me}_{3} \mathrm{C}_{6} \mathrm{H}_{2}$ (Mes)

$\mathrm{NHC8} \quad \mathrm{R}_{1}=\mathrm{R}_{2}=2,6-\mathrm{Pr}_{2} \mathrm{C}_{6} \mathrm{H}_{3}$

$\begin{array}{lll}\mathrm{NHC10} & \mathrm{R}_{1}=\mathrm{Me} ; \mathrm{R}_{2}=\mathrm{Et}\end{array}$

NHC12 $\mathrm{R}_{1}=\mathrm{CH}_{2} \mathrm{Ph}_{2}=\mathrm{Bu}$ (BMIM)

$\mathrm{NHC13} \quad \mathrm{R}_{1}=\mathrm{CH}_{2} \mathrm{Ph} ; \mathrm{R}_{2}=\mathrm{Et}$

$\mathrm{NHC14} \quad \mathrm{R}_{1}=\mathrm{CH}_{2} \mathrm{Ph} ; \mathrm{R}_{2}=\mathrm{PPr}$

$\begin{array}{ll}\mathrm{NHC15} & \mathrm{R}_{1}=\mathrm{PPr} ; \mathrm{R}_{2}=\mathrm{PhOCH}_{3} \\ \mathrm{NHC16} & \mathrm{R}_{1}=\mathrm{Me} ; \mathrm{R}_{2}=\mathrm{CH}_{2} \mathrm{OC}_{10} \mathrm{H}\end{array}$

$\mathrm{NHC16} \mathrm{R}_{1}=\mathrm{Me} ; \mathrm{R}_{2}=\mathrm{CH}_{2} \mathrm{OC}_{10} \mathrm{H}$

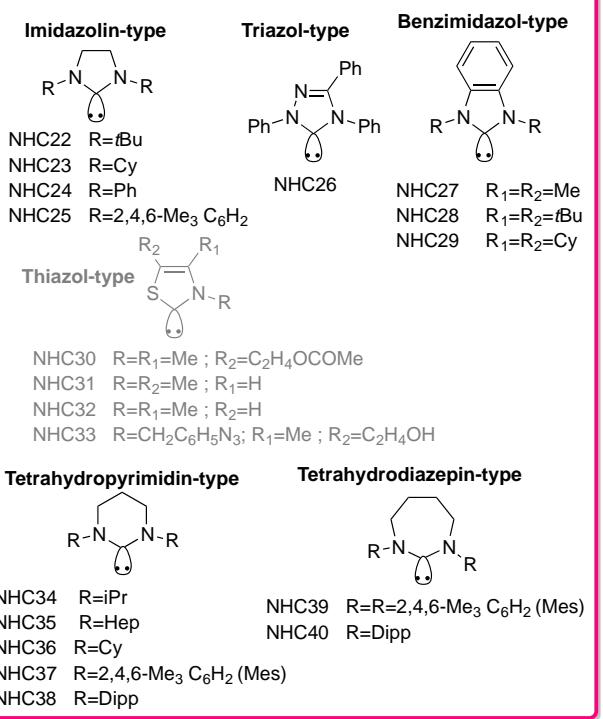

Figure 2. Representative families of organic molecules used as catalysts or initiators for organopolymerization (in gray: compounds already discussed in ref [14]; in black: more recently employed organocatalysts). 

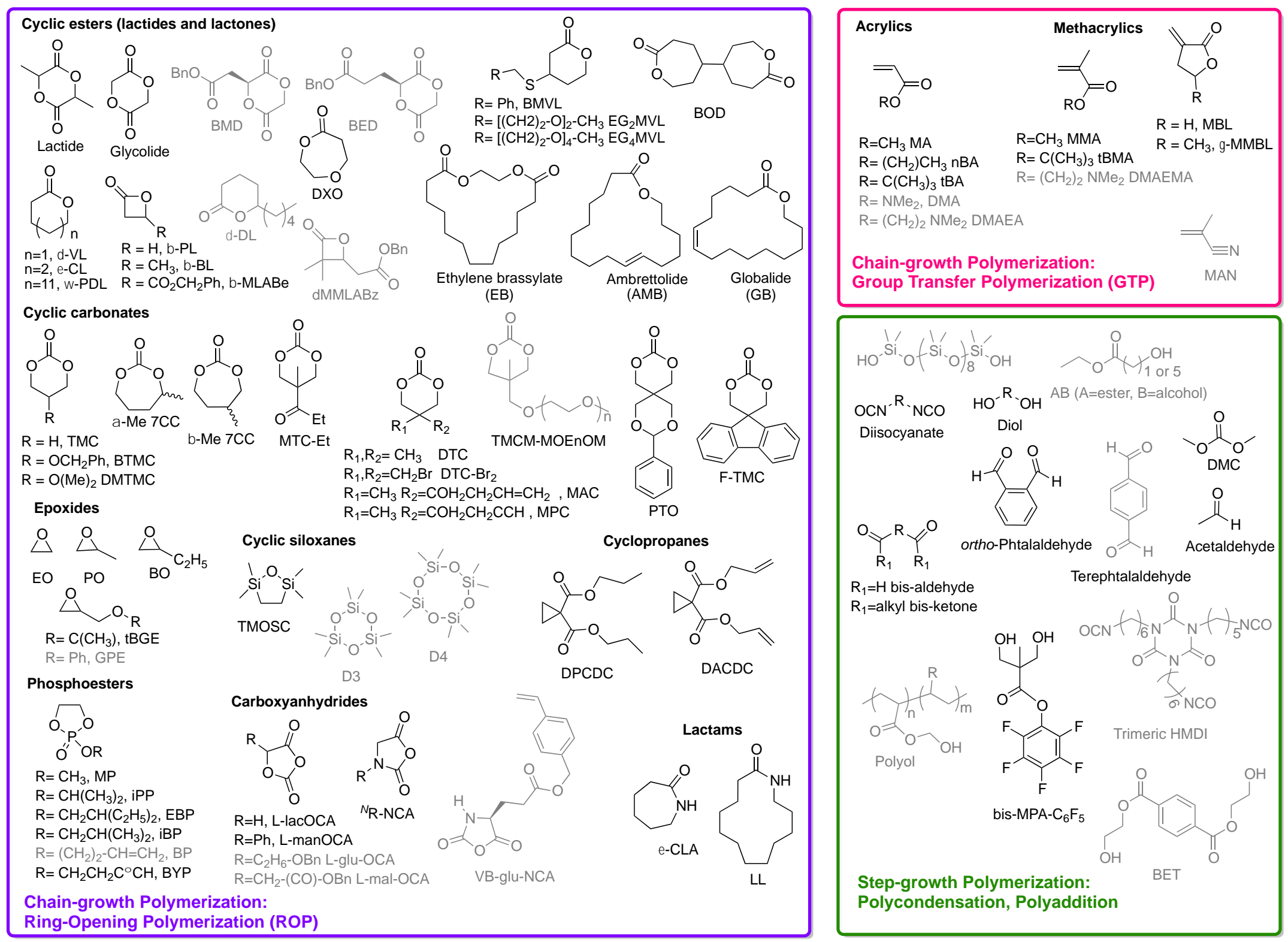

Figure 3. Representative monomers that were subjected to an organopolymerization pathway (in gray: monomers already discussed in ref [14]; in black: monomers polymerized more recently by an organocatalytic pathway). Fig updated 
The resulting activated monomer (1 or $\mathbf{3}$, Scheme $1 \mathrm{a}-\mathrm{b}$ ) being more electrophilic than the monomer itself, it can undergo a nucleophilic addition of the initiator/propagating chain-end (2 or 4 in Scheme 1a-b), resulting in the ring-opening of the monomer while regenerating the catalyst.

In a Lewis-acid-catalyzed polymerization of alkyl (meth)acrylates (e.g. methyl methacrylate, MMA), a Lewis acid, $\mathrm{E}^{*}$ in Scheme 1c (e.g. trimethylsilyltrifluoromethanesulfonimide, $\mathrm{Me}_{3} \mathrm{SiNTf}_{2}$ ) activates the monomer by coordination onto the carbonyl group, forming the intermediate 5 (Scheme 1c). Subsequent 1,4-nucleophlic addition of the initiator (e.g. a trialkylsilyl ketene acetal, SKA) onto 5 leads to a propagating trialkylsilyl enolate 6 (Scheme 1c), where the silyl group has been transferred from the SKA initiator to the growing chain end.

a)<smiles>CC(C)CC1OC(=O)C(C)OC1=O</smiles>

b)

(LA)

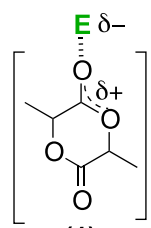

(1)

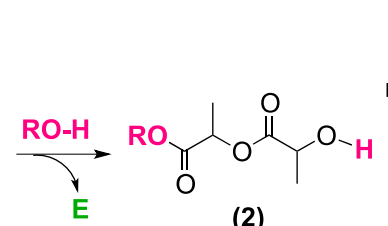

(2)
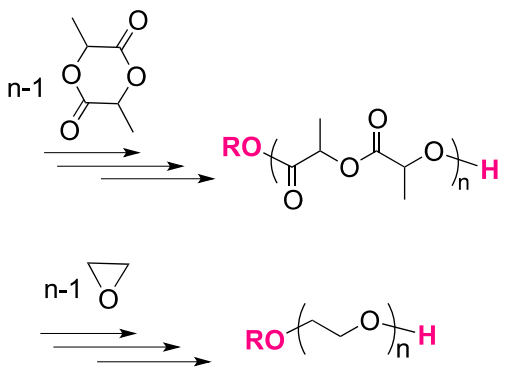

(4)<smiles>[R5]OC(OC)=C(C)C</smiles>

c) thylene oxide (EO) $\underbrace{\stackrel{E}{E} \delta-}_{(3)}$<smiles>O=C(O)C(=O)O</smiles><smiles>CC=C(C)C(=O)OCC</smiles>
methyl methacrylate (MMA)

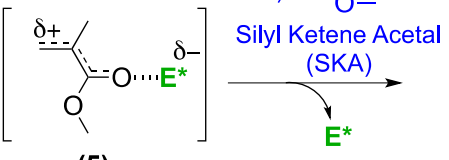

(5)

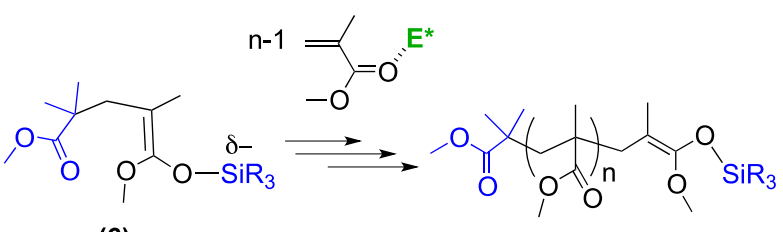

(6)

Scheme 1. Electrophilic AMM mechanism for: a) the ROP of cyclic esters (e.g. LA); b) the ROP of cyclic ethers (e.g. EO); and c) the GTP of (meth)acrylates (e.g. MMA); E and $\mathrm{E}^{*}$ represent a Brønsted and a Lewis acid, respectively.

Nucleophiles $(\mathrm{Nu}) \mathrm{s}$, such as amines, phosphines or NHCs can directly add onto some heterocyclic monomers (e.g. LA or EO), yielding an activated monomer in the form of a zwitterionic alkoxide-type intermediate upon ring-opening (7, Scheme 2). Protonation of $\mathbf{7}$ by an alcohol initiator $(\mathrm{ROH})$ leads to 8 , which undergoes a displacement of $\mathrm{Nu}$ in $\alpha$-position by the alkoxide counter-anion. The ensuing dormant mono-addition product 2 can then propagate the polymerization by reacting with 7, upon proton transfer from 2 to 7.

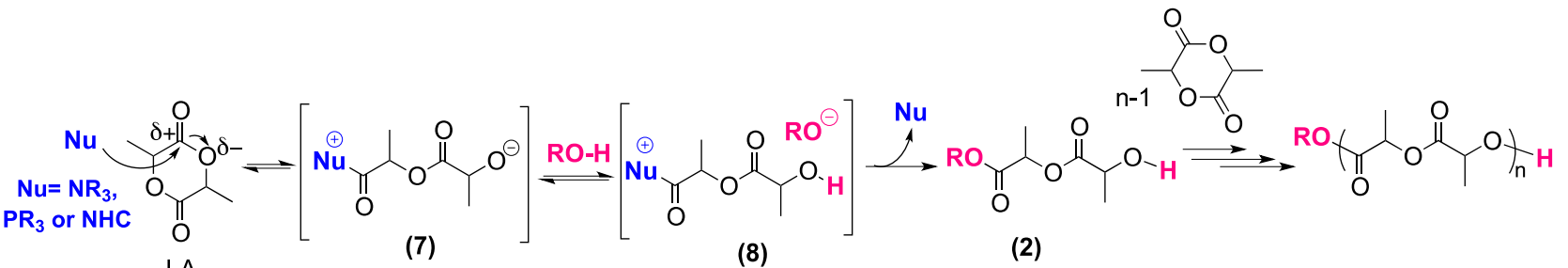

LA

(2)

Scheme 2. Nucleophilic AMM for the ROP of cyclic monomers (e.g. LA) in presence of $\mathrm{ROH}$ as initiators. 
In absence of the alcohol (see section 7), the nucleophile can remain bound to the polymer chain (e.g. in the case of $\beta$-butyrolactone $\beta$-BL), leading to a non-catalytic process (Scheme 3a). In the same way, (meth)acrylate monomers, such as MMA, can directly be polymerized by some Lewis bases (e.g. NHCs), in absence of any other external initiating species. The reaction proceeds via successive and selective 1,4-conjugate additions (via the Michael addition reaction) of the Lewis base (e.g. NHC) onto the monomer, forming a zwitterionic enolate intermediate 10 that can further propagate (Scheme $3 \mathrm{~b}$ ). In the latter case also, the process is not catalytic, the Lewis base acting as a direct organic initiator.

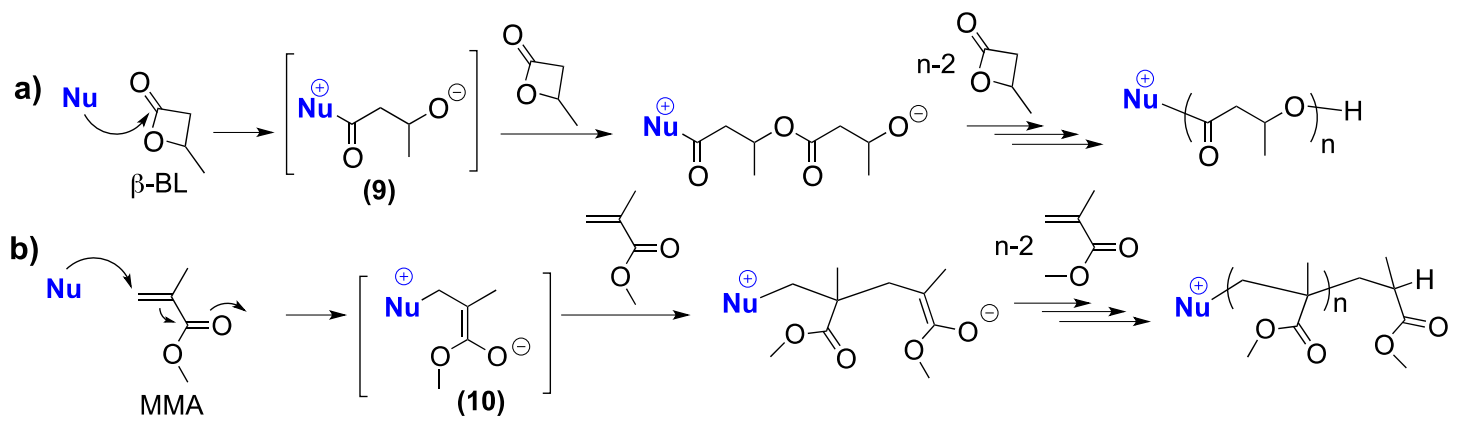

Scheme 3. Nucleophilic and non-catalytic AMM mechanism (see also section 7): a) in zwitterionic ROP (ZROP) of cyclic monomers (e.g. $\beta-\mathrm{BL})$; b) in the zwitterionic polymerization (ZP) of (meth)acrylates (e.g. MMA).

\subsubsection{Initiator/chain-end activated mechanism (ACEM)}

In the ACEM, interaction of an initiating alcohol or of polymer chain-ends with a catalyst (Brønsted basic or H-bonding catalysts) leads to an activated initiating species 11 (Scheme 4a), which is nucleophilic enough to add and ring-open the heterocyclic monomer. The ensuing hydroxyl-terminated mono-adduct 12 can then propagate the polymerization by subsequent ring-openings of the monomer upon activation with the catalyst. The structure of the initiating and propagating species, 11 and $\mathbf{1 2}$, can be described by two limit forms, that are, an activated alcohol-Brønsted base complex and an alkoxide (with a bulky and soft counter cation), depending on the pKa difference between the alcohol and the base (Scheme 4a). Chain growth can then occur by repeated nucleophilic additions of the propagating chain end onto incoming monomers.

In the context of the GTP of (meth)acrylics, organic catalysts such as NHCs or phosphazenes have been reported to promote the polymerization in presence of a SKA initiator.[10] Activation of the SKA would rely on the coordination of the Lewis base to the silicon center. The ensuing hypervalent SKA-based intermediate being more nucleophilic, it would allow the 1,4-addition to proceed. Depending on the nature of the Lewis base, GTP may either proceed by an associative mechanism (via formation of hexacoordinate siliconate species, 14a) or through a dissociative mechanism (via formation of true enolates anions, 14b).[10] 
a)
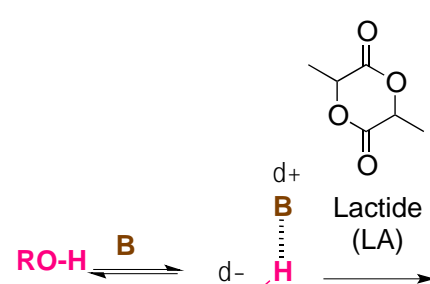

(12)

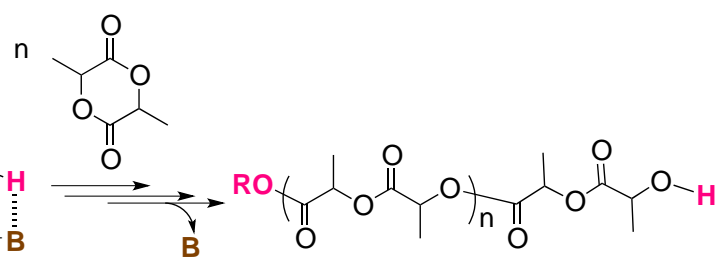

b)

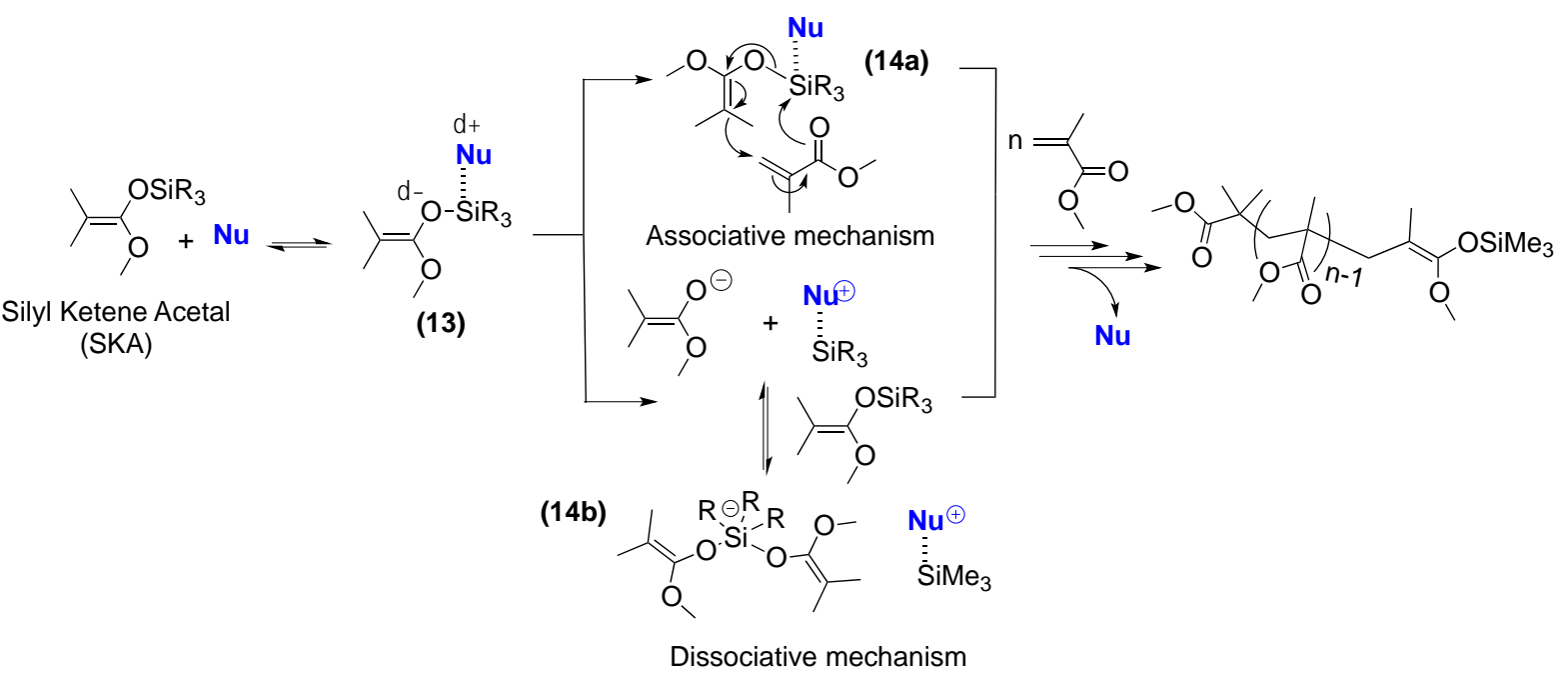

Scheme 4. Basic actived initiator/chain-end mechanism (ACEM) in: a) the ROP of cyclic monomers and b) the associative or dissociative GTP of (meth)acrylates; $B$ is a Brønsted base and $\mathrm{Nu}$ is a nucleophile (Lewis base).

\subsubsection{Bifunctional activation}

Cooperative dual activation of both the monomer and the initiator/chain-end can be achieved with specific organic catalysts, possessing Brønsted acidic/basic properties and/or a $\mathrm{H}$-bonding capability (e.g. thiourea). For instance, a combination of a weak Brønsted acid (E), such as diphenyl phosphate (DPP), and a Brønsted base (B), such as dimethylaminopyridine (DMAP), can be employed in a bicomponent catalytic system to activate a cyclic monomer (Scheme 5a). Such a bifunctional activation is generally triggered using molecules capable of developing hydrogen bonding (e.g. DMAP, thiourea-amino derivatives or the guanidine named 1,5,7-triazabicyclo[4.4.0]dec-5-ene, TBD).[7, 14] A synergy between the two components is expected, providing that the pKa difference between $E$ and $B$ entities is not too high. Very recently, this dual bifunctional activation has been extended to Lewis acid/Lewis base systems. In particular, boranes such as $\mathrm{B}\left(\mathrm{C}_{6} \mathrm{~F}_{5}\right)_{3}$ combined with phosphines such as $\mathrm{P}(t \mathrm{Bu})_{3}$ have been shown to efficiently initiate the polymerization of some methacrylate monomers, via an electrophilic activation of the monomer by the borane and subsequent 1,4-addition of the Lewis base (Scheme 5b).[23] 
a)

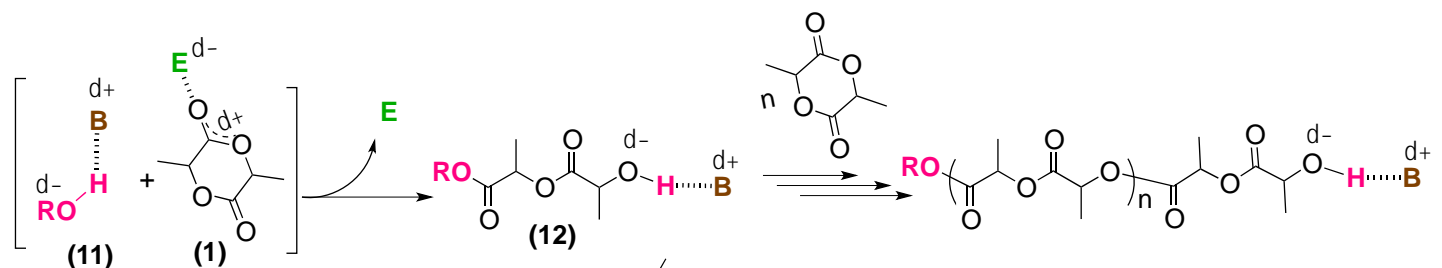

(1)

b)

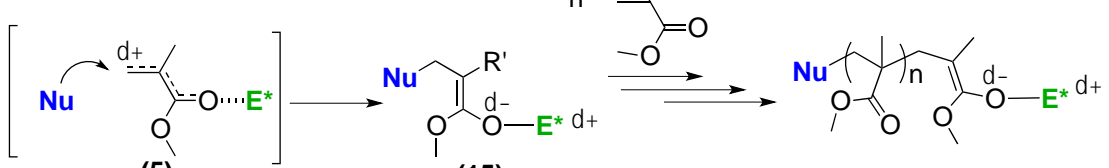

(5)

(15)

Scheme 5. Bifunctional activation mechanism AM/ACEM in: a) the ROP of cyclic monomers and b) the GTP of MMA; E and E* represent a Brønsted and a Lewis acid, respectively; $\mathrm{B}$ is a Brønsted base and $\mathrm{Nu}$ is a nucleophile (Lewis base).

The following sections outline the use of each family of organic catalysts for polymerization reactions, in the presence of an external molecule playing the role of the initiator (= organocatalyzed polymerizations).

\section{Polymerization catalyzed by Brønsted and Lewis acids}

As mentioned above, acid-promoted polymerizations mainly proceed through an electrophilic activation of the monomer, i.e. by a pseudo-cationic mechanism, using protic compounds (typically alcohols) as initiators. It is important to note that some of these acids have been reported to behave as dual catalysts, and this specific activation mode will be detailed in section 6. The ring-opening polymerization (ROP) of cyclic monomers, including esters, carbonates, siloxanes and epoxides, has been the most investigated, using a wide range of sulfonic or sulfonimide Brønsted acids (see Figure 4). Lately, phosphoric acid derivatives have also emerged as efficient promoters of these polymerizations. In contrast to ROP, only a few examples of acid-catalyzed group transfer polymerization (GTP) of (meth)acrylates and stepgrowth polymerizations have been reported. In the following lines, polymerization reactions will be described according to the nature of the functional group of these acid organocatalysts (i.e. sulfonic, sulfonimide, phosphonic, carboxylic-derivatives, etc.), that in fine governs their relative acidity. 


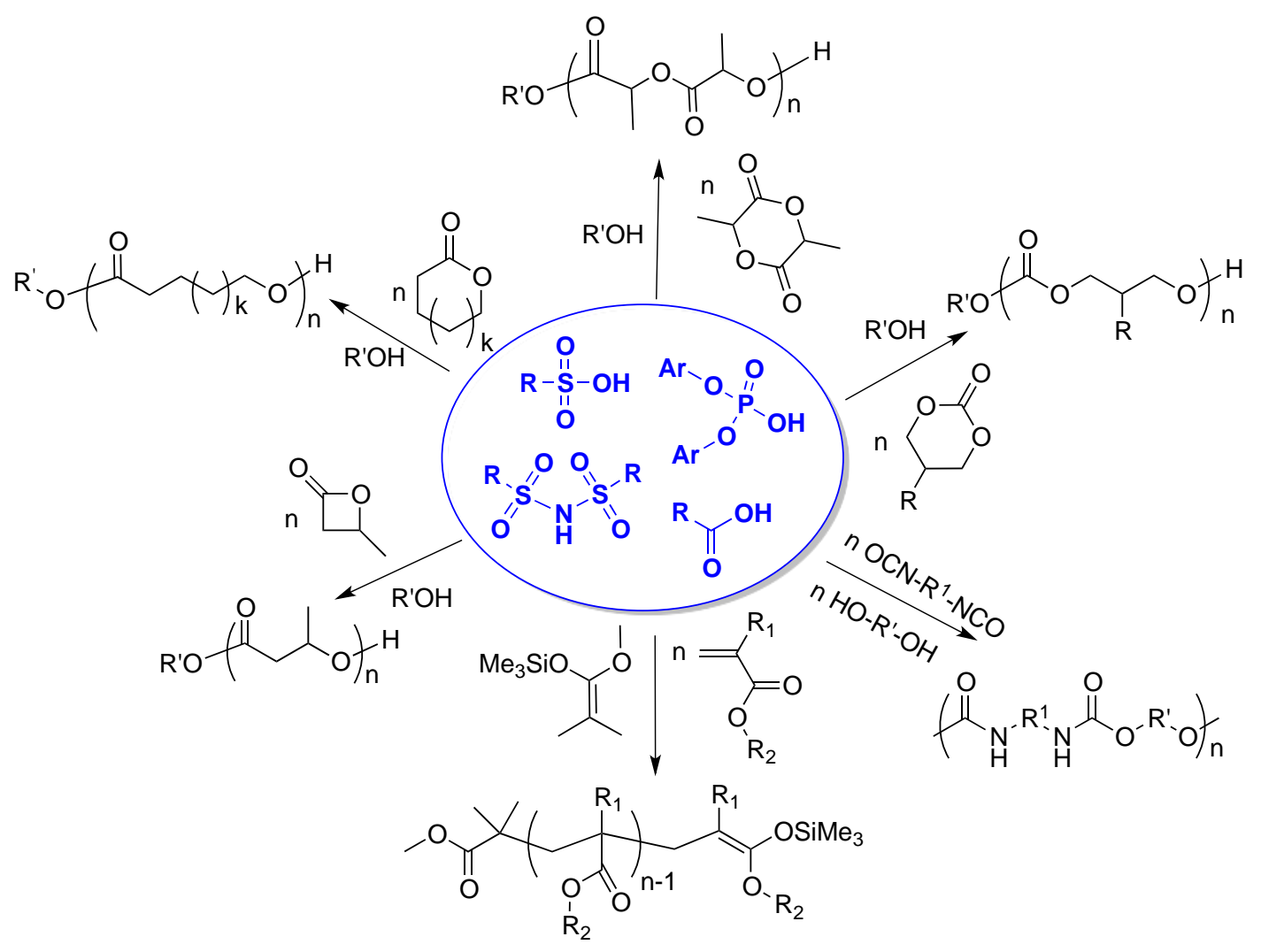

Figure 4. Overview of polymers synthesized by an organic acid catalysis.

\subsection{Sulfonic and sulfonimide acids}

Strong and "super strong" sulfonic acids have been widely investigated to promote the ROP of cyclic esters and carbonates, e.g. lactide, $\varepsilon$-caprolactone $(\varepsilon-C L), \delta$-valerolactone $(\delta-V L)$, and trimethylene carbonate (TMC). Synthesis of polyesters and polyethers by polycondensation reactions in sulfonic acid-tagged imidazolium ionic liquids (referred to as Brønsted acid ionic liquids, BAILS) has also been described. In this case, the BAIL plays both the role of the solvent and the catalyst.[24, 25] An overview of main acidic catalysts employed and related monomers that have been polymerized in this way is shown in Figure 5.

Sulfonic and sulfonimide derivatives

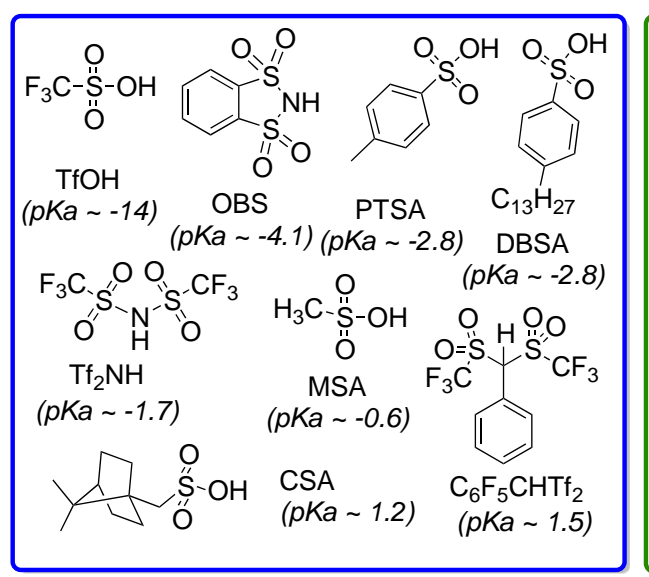

\section{Monomers polymerized}

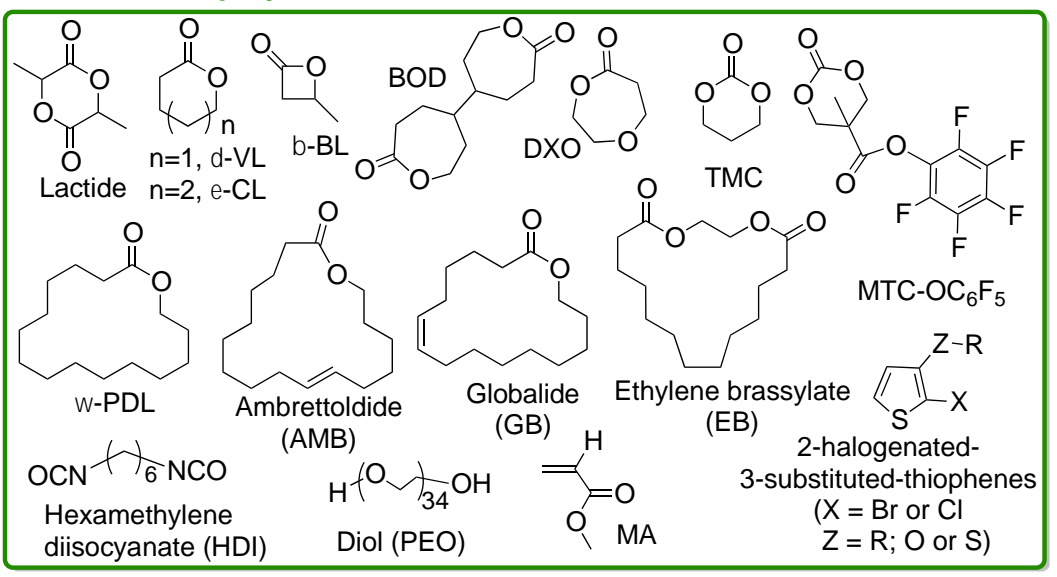

Figure 5. Representative examples of sulfonic and sulfonimide acids derivatives, as well 
as monomers investigated. Note that the strength of these acids is given as an indication (pKa values are given in $\mathrm{H}_{2} \mathrm{O}$ and have been determined using the Hammett acidity function $\left(H_{0}\right)$ )[26], keeping in mind that these values may vary dramatically as a function of the solvent.[27]

Methanesulfonic acid (MSA; pKa - 0.6 in $\mathrm{H}_{2} \mathrm{O}$ )[26] was demonstrated to catalyze the ring-opening copolymerization (ROcP) of both $\varepsilon$ - $C L$ and [4,4'-bioxepane]-7,7'-dione (BOD) in a one pot-fashion, with benzyl alcohol $(\mathrm{BnOH})$ or propargyl alcohol $(\mathrm{PgOH})$ as initiator, in dichloromethane, at r.t (Scheme 6).[28] In this case, BOD played the role of a crosslinking comonomer. Alternatively, the ROP of BOD was also induced by a pre-synthesized hydroxylated poly $(\varepsilon-C L)(P C L-O H)$ as macroinitiator. PCL-based stars consisting of a cross-linked core (nanogel), with a total number average molar masses $\left(M_{n}\right)$ ranging from 9,900 to 36,200 g.mol ${ }^{-1}$ and relatively low dispersity $(\Theta \leq 1.3)$ were thus successfully obtained, from an initial ratio $[\mathrm{BnOH}]_{0} /[\varepsilon-\mathrm{CL}]_{0} /[\mathrm{BOD}]_{0} /[\mathrm{MSA}]_{0}=1: 100: 30: 3$. Although, the polymerization mechanism was not discussed, it was previously postulated by Bourissou, Martín-Vaca et al.[29] that the ROP of $\varepsilon$ CL likely proceeded by an AMM, in presence of $\mathrm{ROH}$ and MSA. With the stronger triflic acid ( $\mathrm{TfOH}$; pKa -14 in $\mathrm{H}_{2} \mathrm{O}$ )[26], however, deactivation of the initiating/propagating alcohol was shown to compete with the activation of the monomer. In other words, the catalytic activity of these organic acids did not simply correlate to their relative acidity.[29]

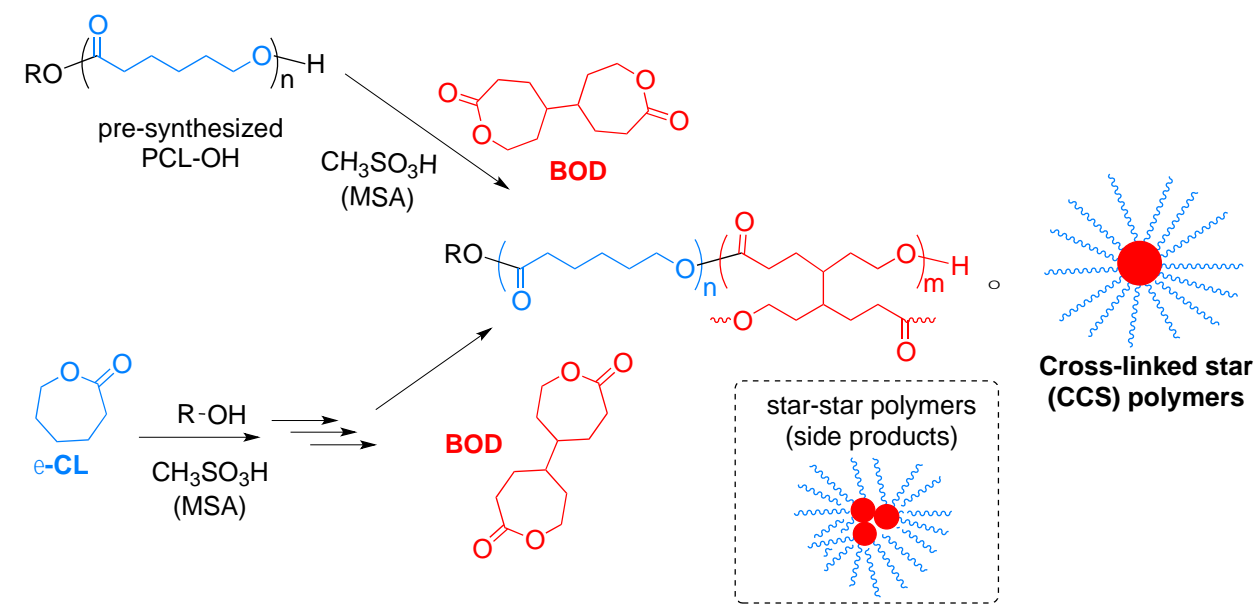

Scheme 6. Synthesis of PCL-based stars via ROP and the " arm-first" approach.[28]

In a more recent study, Zinck et al. investigated the catalytic efficiency of MSA, p-toluenesulfonic acid (PTSA, pKa -2.8 in $\mathrm{H}_{2} \mathrm{O}$ ) and (1R)-(2)-10-camphorsulfonic (CSA, pKa 1.2 in $\mathrm{H}_{2} \mathrm{O}$ ) as organocatalysts ( 1 mol\% relative to the monomer) for the ROP of $\varepsilon$-CL in water (Scheme 7a).[30] The reaction was quantitative at $100{ }^{\circ} \mathrm{C}$, leading to $\mathrm{PCL}$ 's of $\mathrm{M}_{n}$ up to $5,000 \mathrm{~g} \cdot \mathrm{mol}^{-1}(\Theta=1.7-1.9)$. The same reaction conducted at lower temperatures $\left(25\right.$ or $\left.50{ }^{\circ} \mathrm{C}\right)$, using polysaccharides such as dextran or methylcellulose as macroinitiators and PTSA as catalyst, afforded water-soluble polysaccharide-graft-PCL copolymers of $M_{n}=6,200-8,700 \mathrm{~g} \cdot \mathrm{mol}^{-1}$ (Scheme $7 \mathrm{~b}$ ).[30] The authors proposed that the polymerization was 
initiated by 6 -hydroxyhexanoic acid formed upon hydrolysis of the lactone, leading to either a ROP of $\varepsilon-C L$ or to a polycondensation mechanism (Scheme 7).

a)

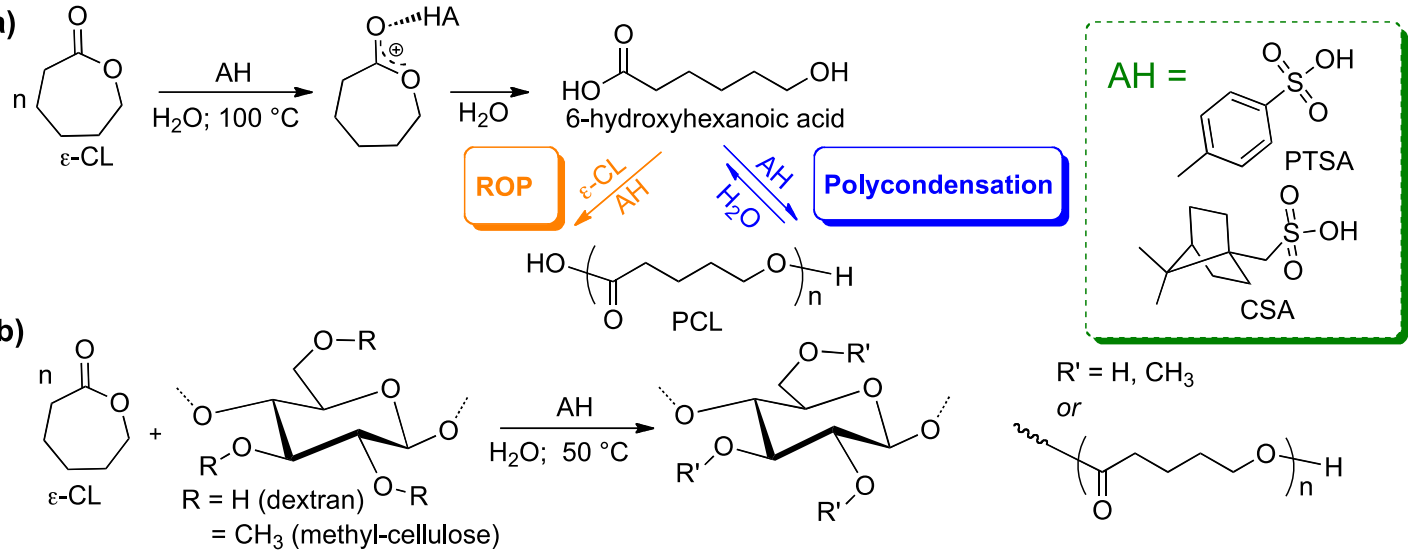

Scheme 7. Acid-catalyzed polymerization of $\varepsilon$-CL in water.[30]

The ROP of $\beta$-butyrolactone ( $\beta-\mathrm{BL}$ ) initiated by iso-propanol (iPrOH) was shown to be catalyzed by $\mathrm{TfOH}(20-40 \mathrm{~mol} \%$ relative to $\mathrm{iPrOH})$ in dichloromethane at r.t.[31] The polymerization was controlled for a degree of polymerization (DP) lower than 30 , producing poly( $\beta$-butyrolactone)s (PBL's) of $\mathrm{M}_{\mathrm{n}}$ equal to $2,400 \mathrm{~g} \cdot \mathrm{mol}^{-1}(\Theta=1.3)$. The observed deviation from linearity at higher monomer to initiator ratios was attributed to the formation of cyclic oligomers by end-to-end ring closure. The same catalytic system was further used for the direct and one-pot ROcP of $\beta-B L$ and L-lactide (LLA). Interestingly, this led to gradient copolymers of molar masses $M_{n}=1,400-3,500 \mathrm{~g} \cdot \mathrm{mol}^{-1}(\Theta=1.3-1.7)$, owing to the different reactivity of the two monomers, PBL being formed during the first stage.

Catalytic activities of MSA and TfOH were compared for the ROP of $\beta$-BL in presence of n-pentanol $(\mathrm{nPenOH})$ as initiator, in benzene at $30^{\circ} \mathrm{C}$.[32] With an initial ratio of acid/ROH $=3 / 1$, $\mathrm{TfOH}$ proved significantly more active than MSA, in relation with its higher acidity $\left(\mathrm{pKa}^{\mathrm{TfOH}} \sim-14\right.$ vs. pKaMSA $\sim$-0.6). Indeed, 20 eq. of $\beta$-BL could be converted within only 15 min with $\mathrm{TfOH}$, whereas 60 min were required with MSA, leading to PBL of controlled molar masses $\left(M_{\mathrm{n}}\right.$ up to 8,200 g.mol $\left.{ }^{-1}\right)$ and narrow distributions $(\Theta<1.25)$. A variety of well-defined di-, tri- and pentablock copolymers were also prepared using dihydroxylated initiators (i.e. butan-1,4-diol) or macroinitiators (i.e. dihydroxylated poly(ethylene oxide) HO-PEO-OH or $\alpha, \omega$-dihydroxylated poly(butadiene) HO-PB-OH), upon successive addition of $\beta$-BL and $\varepsilon$-CL.

The ROP of $\varepsilon$-CL, 1,5-dioxepan-2-one (DXO) and racemic (rac-LA) was also achieved in dichloromethane at r.t., using 3-phenyl-1-propanol (PPA) as initiator and bis(trifluoromethane)sulfonimide $\left(\mathrm{Tf}_{2} \mathrm{NH}, \mathrm{pKa} \sim-1.7\right.$ in $\left.\mathrm{H}_{2} \mathrm{O}\right)$ [33] as catalyst (0.1-3 eq.)[34] Under these conditions, PCL's, poly(1,5-dioxepan-2-one)s (PDXO's), and PLA's of molar masses $\left(M_{n}\right)$ up to $56,500 \mathrm{~g} \cdot \mathrm{mol}^{-1}(\Theta=1.14-1.19)$ were synthesized.

In contrast to MSA, TfOH and $\mathrm{Tf}_{2} \mathrm{NH}$, o-benzenedisulfonimide (OBS; pKa -4.1 in $\left.\mathrm{H}_{2} \mathrm{O}\right)[35]$ was presented as a non-toxic, non-volatile and non-corrosive strong acid. Its catalytic performance in the ROP of $\delta-\mathrm{VL}$ and $\varepsilon-C L$ was studied, using $\mathrm{BnOH}$ as initiator and toluene as 
solvent.[36] The polymerization proceeded at $30^{\circ} \mathrm{C}$ in a controlled fashion, affording PVL and PCL homopolymers, as well as PVL- $b$-PCL diblock copolymers with $M_{n}=3,000-6,000$ g.mol ${ }^{-1}$ and $\oslash$ values in the range of 1.09-1.2. Overall, the catalytic efficiency of OBS was found equivalent to that of $\mathrm{Tf}_{2} \mathrm{NH}$, making OBS a more sustainable alternative than other strong acids.

While the acid-catalyzed ROP of small and medium ring sized lactones has been widely studied, only a few examples describing the ROP of macrolactones have been reported. Three of such macrolactones, namely, $\omega$-pentadecalactone ( $\omega$-PDL), globalide (GB) and ambrettolide $(\mathrm{AMB})$, were polymerized at $80^{\circ} \mathrm{C}$ in bulk or miniemulsion conditions, using dodecylbenzenesulfonic acid (DBSA, pKa -2,8 in $\mathrm{H}_{2} \mathrm{O}$ ) and $\mathrm{TfOH}$ as catalysts $(0.5-10 \mathrm{~mol} \%$ relative to the monomer) (Scheme 8), as reported by Mecerreyes et al.[37] In bulk, although both catalysts induced relatively fast polymerizations ( $>98 \%$ of conversion after $24 \mathrm{~h}$ ), $\mathrm{TfOH}$ was found the most efficient, yielding polyesters of $M_{n}$ around $10,000 \mathrm{~g} \cdot \mathrm{mol}^{-1}$. However, the high dispersity $(\Theta=2-3)$ observed suggested the occurrence of significant transesterification side reactions. In contrast, only DBSA was active in miniemulsion conditions. Based on ${ }^{1} \mathrm{H}$ NMR analyses, a mechanism involving the polycondensation of the hydroxyl-carboxylic ring-opened macrolactone was actually proposed, only oligoesters $\left(M_{n}=2,000 \mathrm{~g} \cdot \mathrm{mol}^{-1}\right)$ being produced under these conditions. The inactivity of $\mathrm{TfOH}$ in waterborne miniemulsion was attributed to the strong solvation of the proton by water molecules, restricting monomer activation.

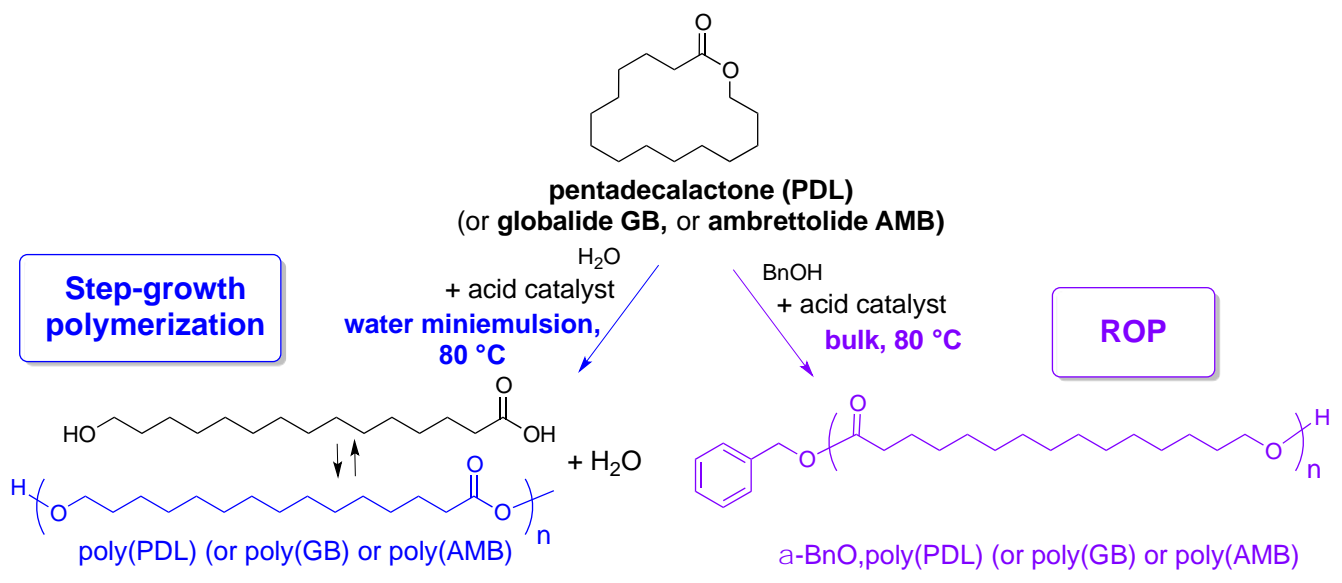

Scheme 8. Acid-catalyzed polymerization of macrolactones in bulk and miniemulsion. [37]

Later on, the same group reported the acid-catalyzed ROP of the renewable macrolactone ethylene brassylate (EB, see Figure 5), both in bulk and in toluene solution, using $\mathrm{BnOH}$ (1 eq.) as initiator.[38] Poly(ethylene brassylate)s of molar masses $M_{n}=5,900 \mathrm{~g} \cdot \mathrm{mol}^{-1}(\Theta=1.9)$ and $M_{n}=2,000 \mathrm{~g} \cdot \mathrm{mol}^{-1}(\Theta=2.7)$ were obtained at $80^{\circ} \mathrm{C}$, in presence of DBSA and PTSA catalysts (1 eq.), respectively. The use of a solvent allowed the viscosity to be decreased, but at the expense of the polymerization rate, $58 \%$ and $73 \%$ of monomer conversion being reached in toluene (96 h) and in bulk (44 h), respectively, for a DP $=100$.

As observed with other macrolactones, although the analysis of chain-ends by MALDIToF mass spectrometry indicated an initiation by $\mathrm{BnOH}$, the presence of species generated by 
inter- and intra-molecular transesterifications were also detected, in agreement with the broad dispersity observed.

Besides cyclic esters, cyclic carbonates have also been subjected to the ROP by an acid catalysis. In 2010, Bourissou et al. first demonstrated the ability of TfOH and MSA to efficiently catalyze the ROP of trimethylene carbonate (TMC), in presence of $n-\mathrm{PenOH}$ as initiator.[39] MSA was found to be a better catalyst than $\mathrm{TfOH}$, avoiding side decarboxylation reactions, presumably due to its lower acidity. However, despite its higher selectivity, two distinct polymer populations (denoted as $\mathbf{A}$ and $\mathbf{B}$ in Scheme 9) were observed by size exclusion chromatography (SEC) with MSA. This was attributed to the competition between two mechanisms consisting in either a ring-opening of the acid-activated monomer by the initiating alcohol (Scheme 9a for population $\mathbf{A}$ ), or a ring-opening of the acid-activated monomer by a non-activated monomer (Scheme 9b for population B).

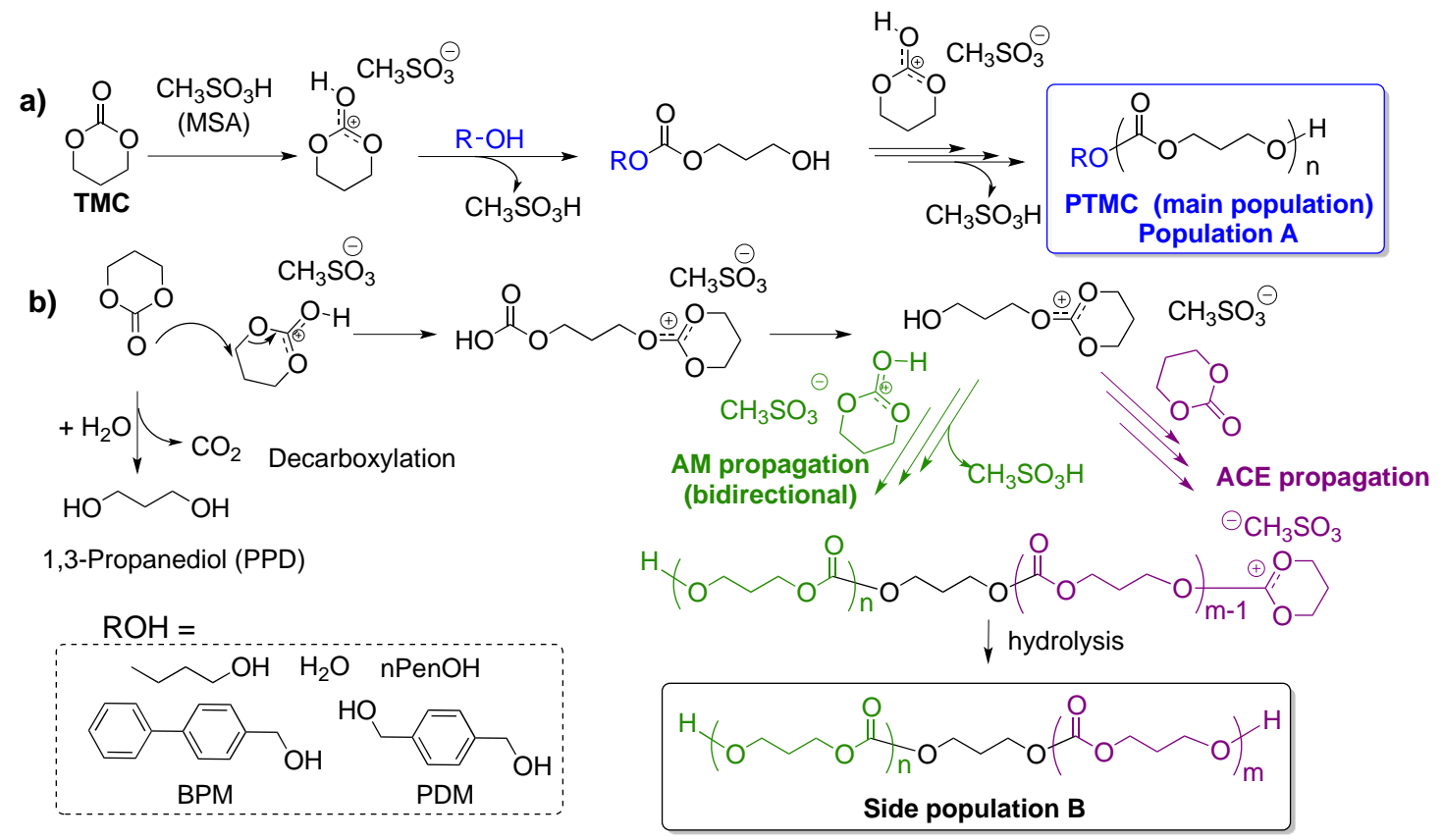

Scheme 9. ROP of trimethylene carbonate catalyzed by methanesulfonic acid: a) AMM with mono-alcohol; b) AMM/ACEM combined bidirectional mechanism.[39, 40]

The MSA-catalyzed ROP of TMC was next investigated by Ribeiro, Peruch et al., using a mono-alcohol (biphenyl-4-methanol, BPM) and a diol (1,4-phenylene-dimethanol, PDM) as initiators, in toluene at $30^{\circ} \mathrm{C}$.[40] With BPM, PTMC's with molar masses ranging from 3,000 to $15,000 \mathrm{~g} \cdot \mathrm{mol}^{-1}(\Theta=1.15-1.25)$ were obtained. The authors also confirmed the occurrence of both the AMM and the ACEM as initially proposed by Bourissou et al.[39] Additionally, they demonstrated that the use of PDM as diol initiating system (a situation where all chain propagations are bidirectional) enabled the preparation of PTMC's exhibiting a unimodal and narrow dispersity $(\Theta=1.07-1.10)$ with $M_{n}$ up to 16,800 g.mol ${ }^{-1}$.

The MSA-catalyzed sequential copolymerization of $\varepsilon$-CL and TMC was then successfully achieved, producing PCL-b-PTMC diblock copolymers of $M_{n}=5,900-10,600 \mathrm{~g} \cdot \mathrm{mol}^{-1}(\bigoplus<1.2)$ 
and PCL- $b$-PTMC- $b$-PCL (ROP of TMC first using $\mathrm{H}_{2} \mathrm{O}$ as initiator) triblock copolymers of $M_{n}=9,100-29,400 \mathrm{~g} \cdot \mathrm{mol}^{-1} \quad(\Theta<1.2) \cdot[41,42]$ Simultaneous polymerization of the two monomers gave rise to PCL-gradient-PTMC copolymers, with a preferential insertion of $\varepsilon-C L$.

To gain a better mechanistic insight into the acid catalyzed ROP of TMC, computational calculations by density functional theory (DFT), including a continuum dielectric representation of dichloromethane, with MSA, TFA or TfOH as catalysts, and methanol as model initiator, were realized by Coady, Hedrick et al..[43] Theoretically, in the case of MSA, only the AMM was predicted to be operative instead of the two distinct and competitive mechanisms observed experimentally by Bourissou et al.[39] and Peruch et al.[40]. Indeed, an activation barrier of $17 \mathrm{kcal}_{\text {. mol}}{ }^{-1}$ and $45 \mathrm{kcal}_{\mathrm{mol}}{ }^{-1}$ were calculated for the ring-opening of the monomer, in the case of the AMM and ACEM, respectively. Thus, the presence of adventitious water was most likely the cause of the two populations observed in those previous studies, although calculations involving a "real initiator" may decrease the difference of energetic barrier between the two mechanisms. Interestingly, DFT calculation also demonstrated that, instead of promoting the AMM, sulfonic acids could eventually display a bifunctional activity, resulting not only in the activation of the carbonyl moiety of the monomer by protonation, but also in the activation of the propagating hydroxyl group through $\mathrm{H}$-bonding with the sulfonate counter anion (Scheme 10). A similar mechanism was previously reported by Bourissou et al. regarding the $\mathrm{ROH}$-initiated ROP of $\varepsilon-C L$ catalyzed by MSA and TfOH[29, 44] and, more recently, by Mecerreyes et al. for PTSA-catalyzed ROP of the ethylene brassylate macrolactone.[38]

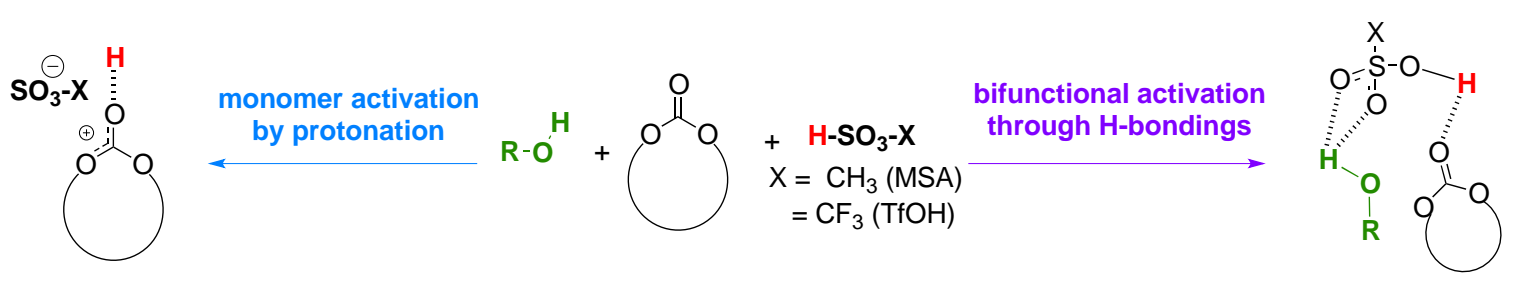

Scheme 10. Acid-catalyzed mechanism for the ROP of cyclic carbonates. Activation by protonation vs. bifunctional activation through $\mathrm{H}$-bondings proposed by Coady, Hedrick et al.[43] and Bourissou et al. [29, 44]

The acid-catalyzed ROP of TMC was also extended to functional carbonates. For instance, the ROP of an activated pentafluorophenyl carbonate, denoted as $\mathrm{MTC}-\mathrm{OC}_{6} \mathrm{~F}_{5}$, using PTSA (pKa -2.8 in water) and TfOH as catalysts (10 eq.) was reported.[45] Alcohol initiators such $\mathrm{BnOH}, \mathrm{PyBuOH}$, as well as a methoxy-terminated poly(ethylene oxide) (MeOPEO-OH) were employed to this end (Scheme 11). Successful ROP of MTC-OC $6 \mathrm{~F}_{5}$ was achieved in dichloromethane at r.t., leading to poly $\left(\mathrm{MTC}-\mathrm{OC}_{6} \mathrm{~F}_{5}\right)$ with $\mathrm{M}_{\mathrm{n}}$ ranging from 5,000 to 37,200 g. $\mathrm{mol}^{-}$ ${ }^{1}(\Xi<1.3)$. Interestingly, no reaction between $\mathrm{ROH}$ and the activated ester was observed under these conditions. When using PTSA, resulting polymers exhibited significantly lower $M_{n}$ values than those expected, indicating a loss of control of the polymerization. These results highlighted 
the unexpected selectivity of those catalysts as a function of their strength and contrasted with those previously reported for the ROP of TMC, where the relatively weak MSA allowed a better control over the polymerization than the stronger $\mathrm{TfOH}$ acid [39]. Poly $\left(\mathrm{MTC}-\mathrm{OC}_{6} \mathrm{~F}_{5}\right)$ could be subsequently post-functionalized, under mild reaction conditions, with a variety of primary and secondary amines, including macromolecular amines such as $\mathrm{PEO}-\mathrm{NH}_{2}$, providing rapid access to a wide range of functional polycarbonates.

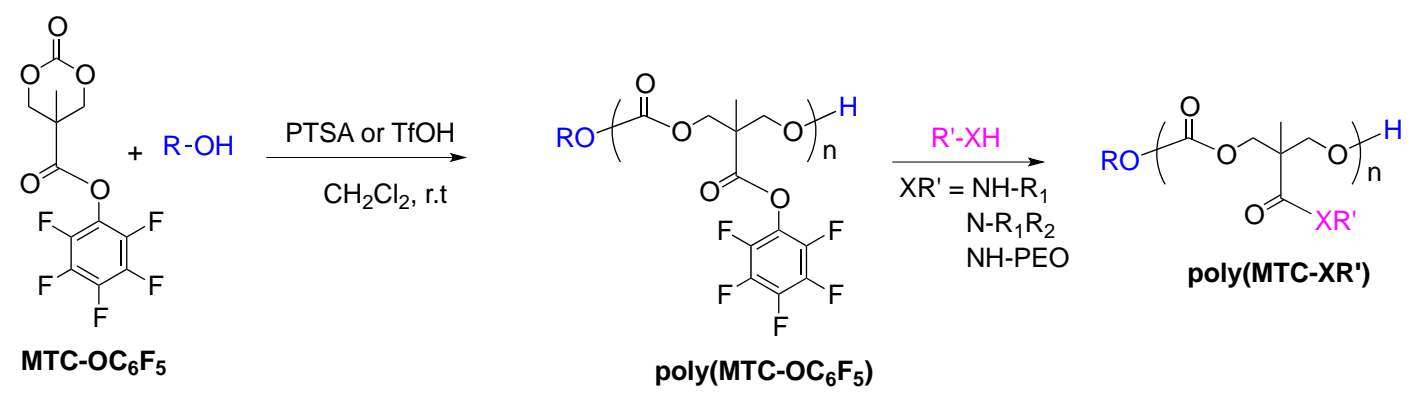

Scheme 11. ROP of MTC-OC $6 \mathrm{~F}_{5}$ catalyzed by $p$-toluenesulfonic acid (PTSA) or triflic acid $(\mathrm{TfOH})$ in presence of alcohol initiator and further post-chemical modification.[45]

Besides ROP, organic acid-catalyzed step-growth polymerization reactions were also implemented. For instance, Hedrick et al. in collaboration with Mecerreyes et al. performed the polyaddition between a bis-hydroxylated PEO and hexamethylene diisocyanate (HDI) in dichloromethane at $20^{\circ} \mathrm{C}$, using MSA and TfOH as catalysts (Scheme 12).[46] These sulfonic acids were highly effective, yielding ( $98 \%$ of monomer conversion) polyurethanes (PUs) of molar masses $\left(M_{w}\right)$ in the range of $18,300-28,200$ g.mol ${ }^{-1}$ and $\Theta=1.3-1.7$.

DFT calculations suggested the occurrence of a dual hydrogen-bonding mechanism, where both the alcohol initiator and the isocyanate were activated by interaction with the sulfonate anion $\left(\mathrm{XSO}_{3}{ }^{-}\right)$and the proton of the catalyst, respectively. Note that protonation of the isocyanate might either occur at the nitrogen or oxygen atoms (see insert in Scheme 12). In both cases, the reaction followed a preference for $\mathrm{N}$-activation over $\mathrm{O}$-activation pathways.

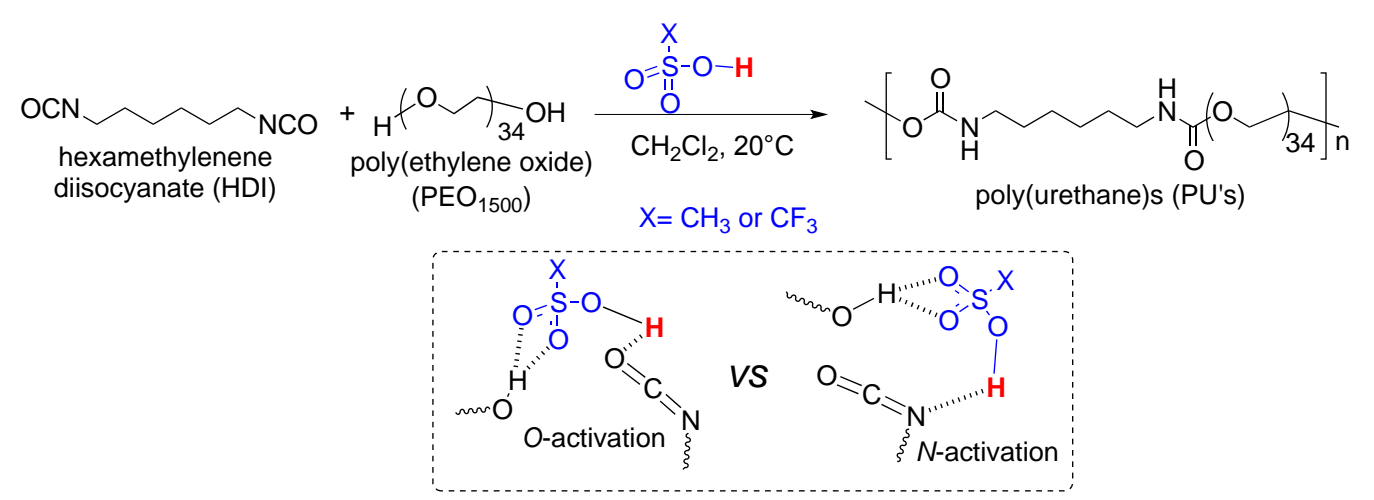

Scheme 12. Organic acid-catalyzed synthesis of PUs from diisocyanate and diol.[46]

As depicted in Scheme 13, the same group reported the post-polymerization modification of PUs derived from such an acid-catalyzed polymerization.[21] Using $5 \mathrm{~mol} \%$ of $\mathrm{TfOH}$, the polyaddition between a pentafluorophenyl ester-containing diol derived from 2,2bis(hydroxymethyl)propanoic acid (bis-MPA), denoted as bis-MPA- $\mathrm{C}_{6} \mathrm{~F}_{5}$, and HDI produced PUs 
of $\mathrm{M}_{\mathrm{n}}$ of $25,000-37,000 \mathrm{~g} \cdot \mathrm{mol}^{-1}(\Theta=1.3-1.6)$. As previously reported,[45] these poly(bis-MPA$\mathrm{C}_{6} \mathrm{~F}_{5}-\mathrm{HDI}$ ) urethanes could also be post-functionalized by various amines, yielding highly functional PU derivatives.

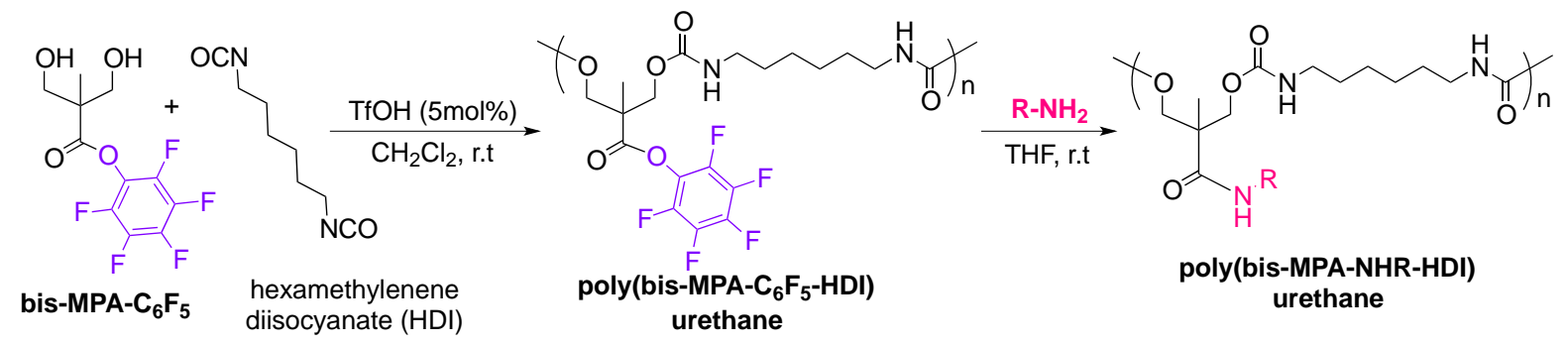

Scheme 13. Synthesis of polyurethanes from bis-MPA- $\mathrm{C}_{6} \mathrm{~F}_{5}$ and hexamethylene diisocyanate using $\mathrm{TfOH}$ as catalyst. [21]

Besides the ROP of cyclic esters and carbonates, methyl acrylate (MA) was reported to undergo an acid-catalyzed GTP, using various trialkylsilyl ketene acetal (SKA) as initiators, such as 1-trimethylsiloxy-, 1-triethylsiloxy-, and 1-triiso-propylsiloxy-1-methoxy-2-methyl-1-propene (MTS $^{\mathrm{Me}}, \mathrm{MTS}^{\mathrm{Et}}$, and MTS ${ }^{\mathrm{iPr}}$, respectively). GTP was performed using $2 \mathrm{~mol} \%$ (relative to the initiator) of pentafluorophenylbis(triflyl)-methane $\left(\mathrm{C}_{6} \mathrm{~F}_{5} \mathrm{CHTf}_{2}\right.$, pKa 1.5 in $\left.\mathrm{AcOH}\right)$.[47, 48] The bulkiest triisopropylsilyl ketene acetal (MTS ${ }^{i P r}$ ) was shown to be the most suitable initiator in minimizing back-biting reactions observed with the other initiators tested. Poly(methyl acrylate)s (PMA's) of $M_{n}=2,900-108,000 \mathrm{~g} \cdot \mathrm{mol}^{-1}$ and narrow distributions ( $\theta=1.03-1.07$ ) were thus obtained in toluene at r.t. Using the same strategy, $n$-butyl acrylate $(n \mathrm{BuA})$ was copolymerized with MA, producing poly(methyl acrylate- $b$ - $n$-butyl acrylate) (PMA- $b$-P $n \mathrm{BuA}$ ) diblock copolymers of $M_{n}=11,500-14,200 \mathrm{~g} \cdot \mathrm{mol}^{-1}(\Theta<1.05)$. According to the authors, the key intermediate species was the silicon Lewis acid, $\mathrm{C}_{6} \mathrm{~F}_{5} \mathrm{C}\left(\mathrm{SiR}_{3}\right) \mathrm{Tf}_{2}$, generated from the reaction between the SKA initiator and $\mathrm{C}_{6} \mathrm{~F}_{5} \mathrm{CHTf}_{2}$. The supposedly Lewis acid-activated monomer mechanism (see insert in Scheme 14) was thus distinct from both associative and dissociative mechanisms proposed for the GTP of (meth)acrylates using nucleophilic catalysts (Scheme 4b).[10]

The more common $\mathrm{N}$-(trimethylsilyl)bis-(trifluoroethanesulfonyl)imide $\left(\mathrm{Me}_{3} \mathrm{SiNTf}_{2}\right.$ ) Lewis acid could also catalyze the GTP of $n B u A$ in toluene at r.t., using MTS ${ }^{\text {iPr }}$ derivatives as functional initiators.[49] Under these conditions, a-functionalized (hydroxyl, ethynyl, vinyl, and norbornenyl) poly(nbutyl acrylate)s (PnBuA's) of $M_{n}=4,000-142,700 \mathrm{~g} \cdot \mathrm{mol}^{-1}$ ( $(\bigoplus=1.03-1.10)$ could be achieved. Moreover, addition of functional 2-phenyl acrylate terminating agents allowed synthesizing, not only $\omega$-functionalized (ethynyl, vinyl, hydroxyl, and bromo), but also as $\alpha, \omega$-functionalized polyacrylates of $M_{n}=4,000-4,800$ g.mol ${ }^{-1}(\Theta=1.06-1.08)$. 

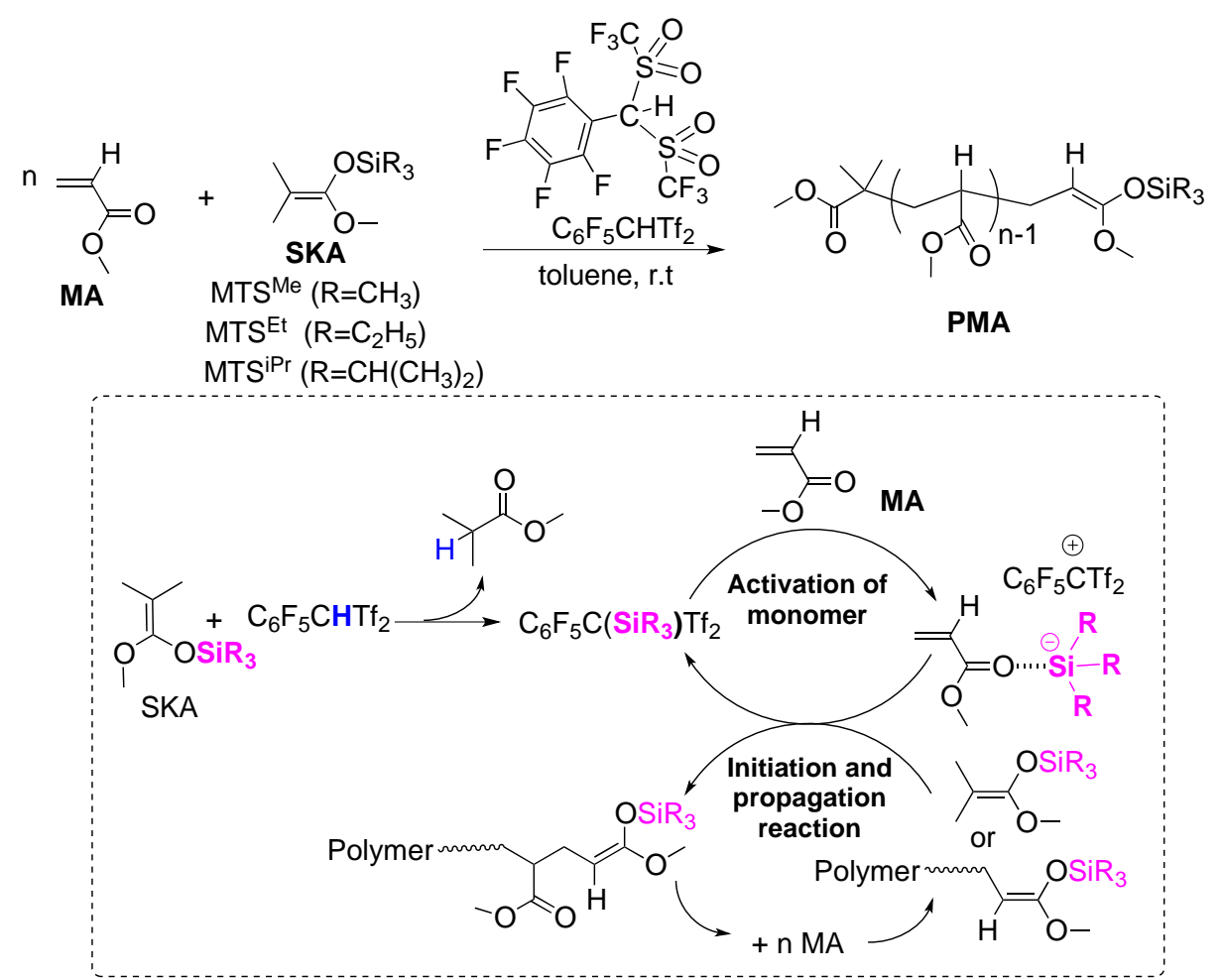

Scheme 14. $\mathrm{C}_{5} \mathrm{~F}_{5}-\mathrm{CHTf}_{2}$-promoted GTP of MA using trialkylsilyl ketene acetals initiators.[48]

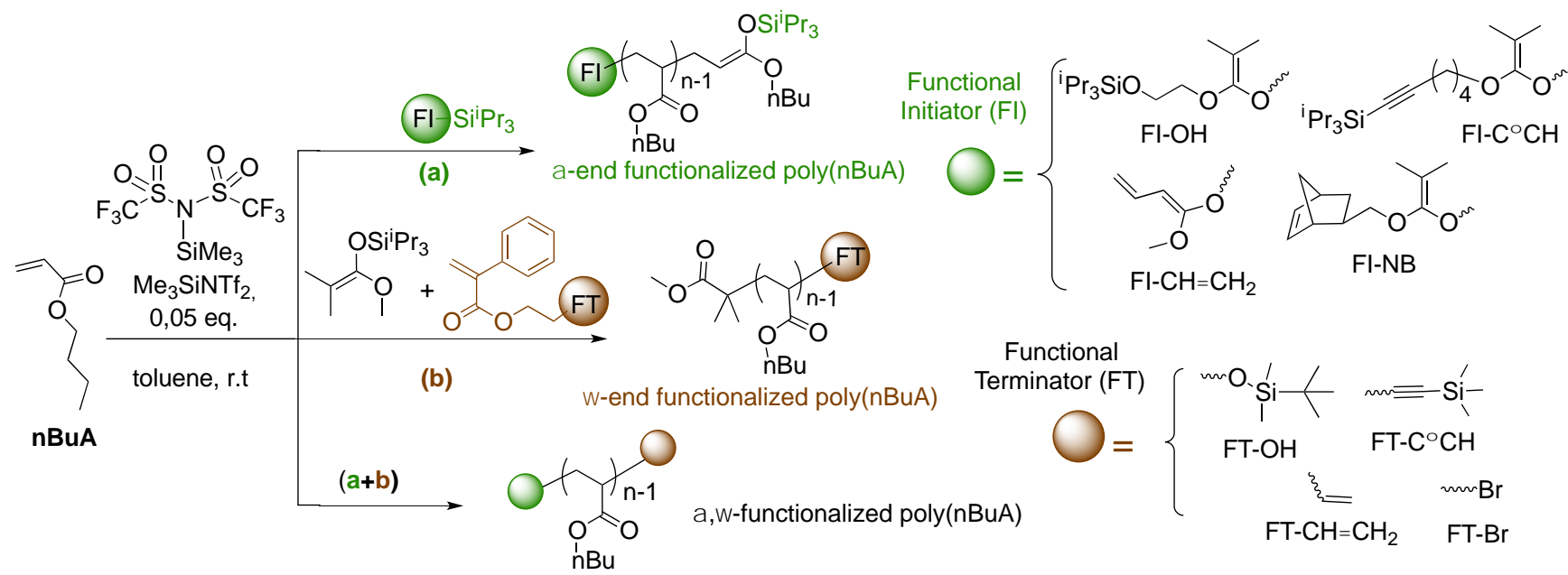

Scheme 15. Synthesis of $\alpha, \omega$-functionalized poly(nBuA)'s by $\mathrm{Me}_{3} \mathrm{SiNTf}_{2}$-catalyzed GTP using functional initiators and terminators.[49]

Triarylsulfonium hexafluorophosphate salts were employed as photoactive acidic catalysts (0.33 mol\% with respect to monomer) for the ROP of $\varepsilon-\mathrm{CL}, \delta$-VL and TMC in propylene carbonate at r.t, using $\mathrm{BnOH}$ as initiator (Scheme 16a).[50] Under UV irradiation, the sulfonium indeed decomposed into sulfide species with concomitant release of the strong $\mathrm{HPF}_{6}$ acid (Scheme 16b), allowing homopolymers of $M_{n}=1,600-27,700 \mathrm{~g} \cdot \mathrm{mol}^{-1}(\nexists=1.09-1.26)$, as well as PVL-b-PLLA- $b$-PVL block copolymers to be prepared in a "one-pot" reaction. However, the bimodal molar mass distribution observed by SEC suggested the occurrence of side reactions, likely resulting from competitive mechanisms (cationic ACEM and $\mathrm{BnOH}$-initiated AMM). It should be emphasized here that these sulfonium hexafluorophosphate salts are not true organic 
catalysts but should rather be considered as pre-catalysts, the actual active species being the inorganic $\mathrm{HPF}_{6}$ acid.

a)
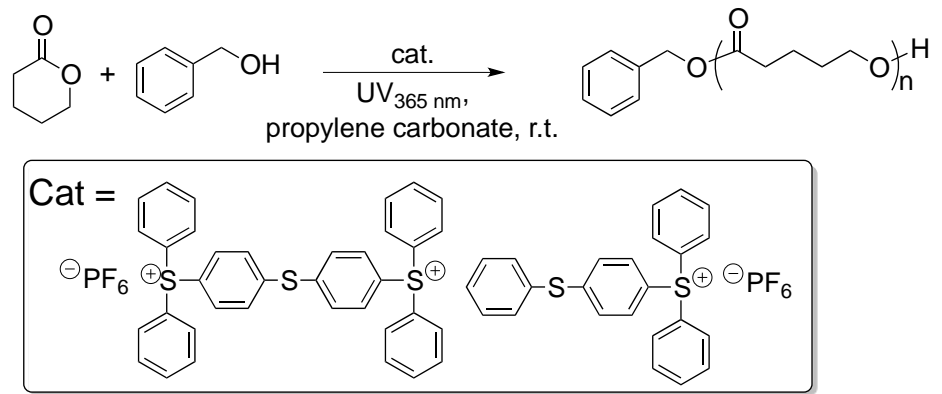

b)

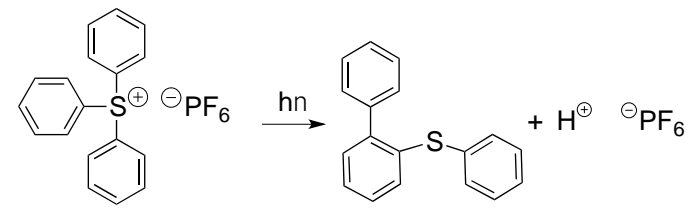

Scheme 16. a) ROP of $\delta$-VL in presence of $\mathrm{BnOH}$ and triarylsulfonium hexafluorophosphate salts; and b) proton generation upon UV irradiation.[50]

Very recently, $\mathrm{TfOH}$ was evidenced to be an effective catalyst for the chain-growth cationic polymerization of 2-halogenated-3-substituted-thiophenes featuring alkyl, alkylthio, alkoxy, and dialkoxy substituent groups (see Figure 5 for related structures).[51] Polythiophenes of $M_{w}=2,500-6,100 \mathrm{~g} \cdot \mathrm{mol}^{-1}(\Theta=1.31-1.91)$ were obtained in tetrachloroethane at $100{ }^{\circ} \mathrm{C}$, with low amounts of $\mathrm{TfOH}(0.05$ eq.), while MSA did not give reproducible results. The chain growth process was proposed to involve nucleophilic aromatic substitution-type reactions, where the nucleophile is a non-activated thiophene and the electrophile is a protonated monomer. Upon loss of $\mathrm{HX}(\mathrm{X}=\mathrm{Br}, \mathrm{Cl})$, the aromaticity is recovered, leading to the conjugation of the polymer units. As expected, the polymerization was found to be dependant on both the strength of the acid and the electron density of the thiophene monomers. For instance, the polymerization of chlorinated monomers proved much slower than that of bromo-analogues. In addition, a copolymer of $M_{w}=7,000 \mathrm{~g} \cdot \mathrm{mol}^{-1}(\Theta=1.63)$ was obtained upon sequential polymerization of 2chloro-3-butylthiothiophene and 2-chloro-3-dodecylthiothiophene (Scheme 17).

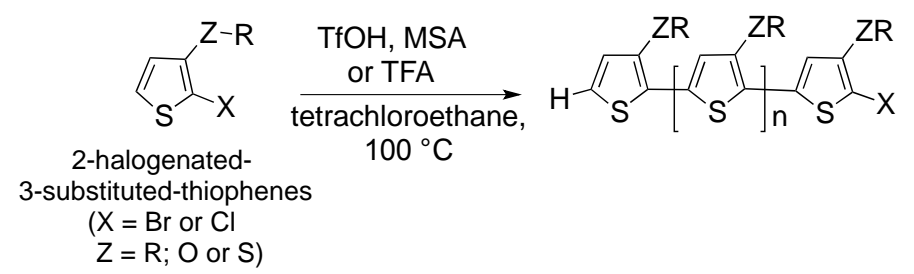

Scheme 17. Brønsted acid-catalyzed polymerization of 2-halogenated thiophenes.[51]

\subsection{Carboxylic acid compounds}

In comparison with sulfonic and sulfonimide acids ( $\mathrm{pKa} \leq 1.5$ in $\mathrm{H}_{2} \mathrm{O}$ ), carboxylic acids and amino-acids are weak acids ( $\mathrm{pKa} \sim 3-5$ in $\mathrm{H}_{2} \mathrm{O}$ ) that have been sparingly investigated as catalysts in polymerization reactions. Related studies have mainly focused on cyclic esters and carbonates as monomer substrates (Figure 6). 

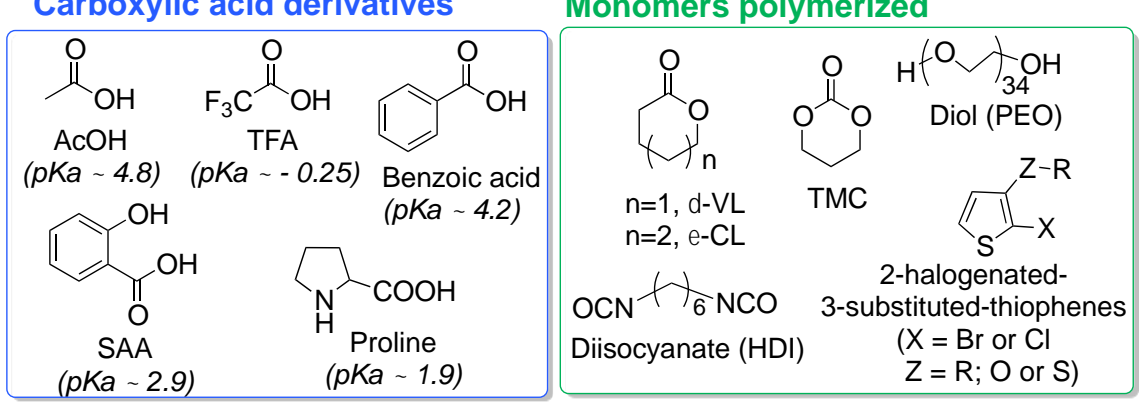

Figure 6. Representative examples of carboxylic acids and monomers employed in organo-catalyzed polymerizations investigated; pKa values are given in $\mathrm{H}_{2} \mathrm{O}$.

For instance, the catalytic activity of salicylic acid (SAA; pKa 2.9 in $\mathrm{H}_{2} \mathrm{O}$ ) was recently investigated for the ROP of $\varepsilon-\mathrm{CL}$ and $\delta$-VL, with $\mathrm{BnOH}$ as initiator (Scheme 18).[52] The polymerization successfully proceeded in bulk at $80^{\circ} \mathrm{C}$, leading to narrowly distributed polyesters with molar masses, $M_{n}$, in the range of $1,300-41,000 \mathrm{~g}^{-\mathrm{mol}^{-1}}(\Theta=1.03-1.16)$ and $M_{n}=3,200-12,100$ g.mol ${ }^{-1}(\Theta=1.05-1.12)$ for PCL and PVL, respectively. Well-defined PCL$b$-PVL diblock copolymers were also synthesized by sequential addition of $\varepsilon-C L$ and $\delta$-VL. In addition, end-functionalized PCL's with vinyl, propargyl and azido groups, as well as PEO- $b$ PCL diblock copolymers could be prepared, using functionalized initiators, such as propargyl alcohol (PgOH), 6-azido-1 hexanol (AHA), 2-hydroxyethyl methacrylate (HEMA), and a methoxy poly(ethylene oxide) (MeOPEO) macroinitiator. Interaction developing between SAA and $\varepsilon-C L$, as observed by NMR, suggested that the polymerization likely proceeded by an AMM (see Scheme 1a), although a bifunctional activation cannot be completely ruled out.

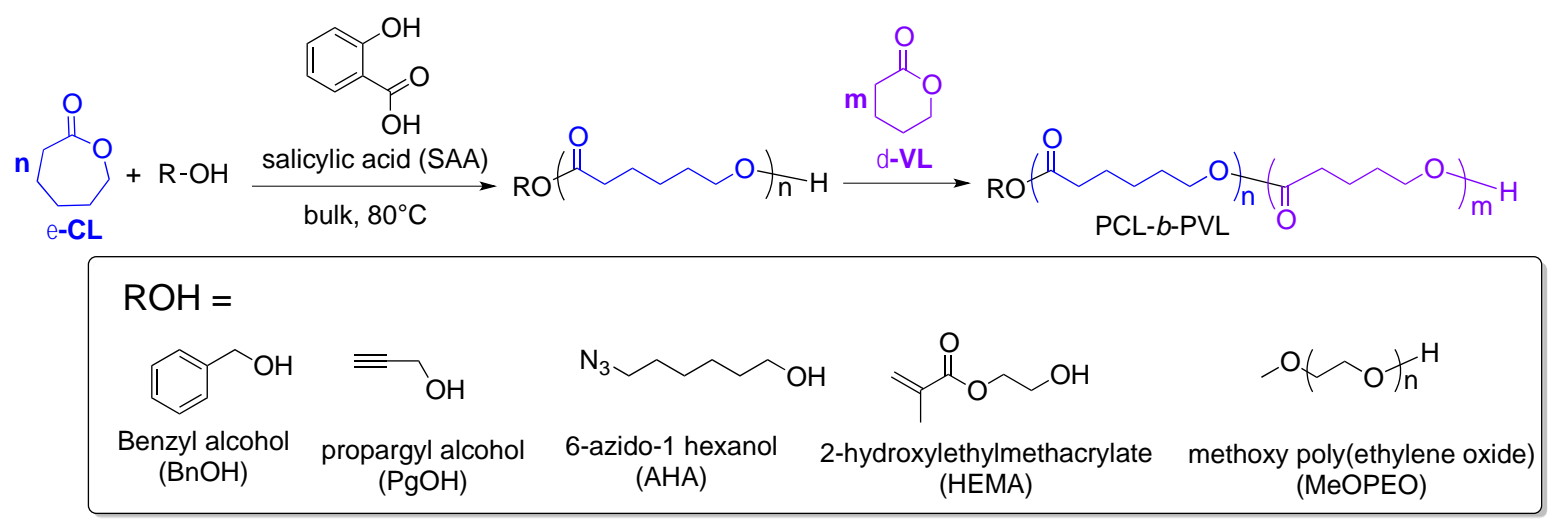

Scheme 18. Salicylic acid (SAA)-promoted ROP of $\varepsilon-C L$ and $\delta$-VL using alcohols as initiators.[52]

Trifluoroacetic acid (TFA, pKa -0.25 in $\mathrm{H}_{2} \mathrm{O}$ ), benzoic acid, and acetic acid $(\mathrm{AcOH}$, pKa $~ 4.2$ in $\mathrm{H}_{2} \mathrm{O}$ ) were studied, both experimentally and computationally, for the ROP of TMC in dichloromethane at r.t., using $\mathrm{PyBuOH}$ as initiator.[43] Experimentally, no catalytic activity was noted with these acids despite the similar energetic profiles calculated for the ring-opening of the monomer by AMM with TFA or MSA as catalyst. In the latter case, instead of the $\mathrm{pK}_{\mathrm{a}}$ 
argument, a bifunctional activation mechanism was put forward, with the acid bound to both monomer carbonyl and propagating hydroxyl group (see Scheme 10 above).

Recently, TFA ( 0.5 eq.) was yet found suitable to catalyze the cationic polymerization of 2-halogenated-3-substituted-thiophene, giving similar results in various halogenated solvents at r.t. In contrast, $\mathrm{AcOH}$ was too weak to initiate the polymerization.[51] Polythiophenes with molar masses, $M_{w}$, in the range of $1,300-3,900 \mathrm{~g} \mathrm{~mol}^{-1}(\Theta=1.18-4.77)$ were obtained from TFA, the dispersity increasing for prolonged reaction times. Polymerization of electron-poor 2bromothiophene was only effective with the most acidic $\mathrm{TfOH}$ catalyst, while some electron-rich monomers, such as 2-bromo-3-alkoxy thiophenes and bromo-ethylenedioxythiophene (EDOT), could be polymerized with TFA. This metal-free methodology affords highly solutionprocessable and conductive poly(alkylthiophene) with a conductivity greater than $180{\mathrm{~S} . \mathrm{cm}^{-1}}^{-}$ [51]

Synthesis of PUs by polyaddition between $\alpha, \omega$ bis-hydroxy poly(ethylene oxide) and HDI was conducted in dichloromethane, at $20^{\circ} \mathrm{C}$, using TFA and $\mathrm{AcOH}$ as catalysts (0.05 eq.).[46] In comparison with sulfonic acids (see Scheme 12 above), carboxylic acids were ineffective, however, monomer conversions less than $3 \%$ being reached after $6 \mathrm{~h}$ of reaction, as a consequence of the low acidity of these catalysts.

\subsection{Phosphoric acid and their derivatives}

Phosphoric acids (pKa 2-4 in DMSO)[53] have attracted a renewed interest as relatively mild Brønsted acids in organocatalysis in general,[54] and in polymerization reactions in particular.[8,55] As highlighted in Figure 7, they can provide a dual activation due to both hydrogen-bonding donating and accepting properties of the acidic $\mathrm{O}-\mathrm{H}$ and basic $\mathrm{P}=\mathrm{O}$ moieties.[55] Although these catalysts could have been discussed in the section devoted to bifunctional catalytic systems, they have been included in the present section to facilitate comparison with the other acidic catalysts. 

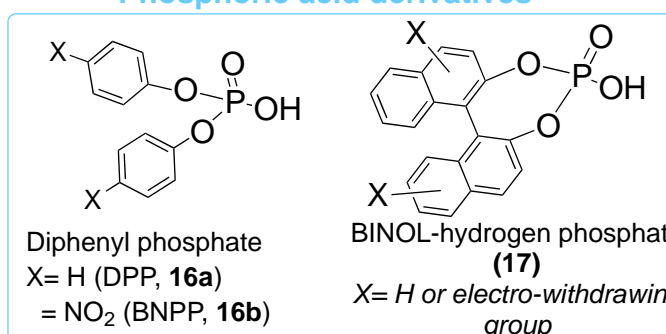

BINOL-hydrogen phosphate (17)

$X=H$ or electro-withdrawing

$=\mathrm{NO}_{2}(\mathrm{BNPP}, 16 \mathbf{b})$

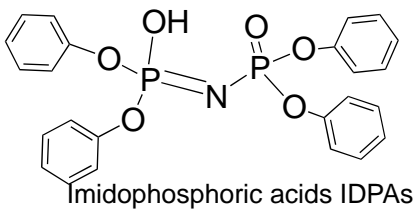

(18)

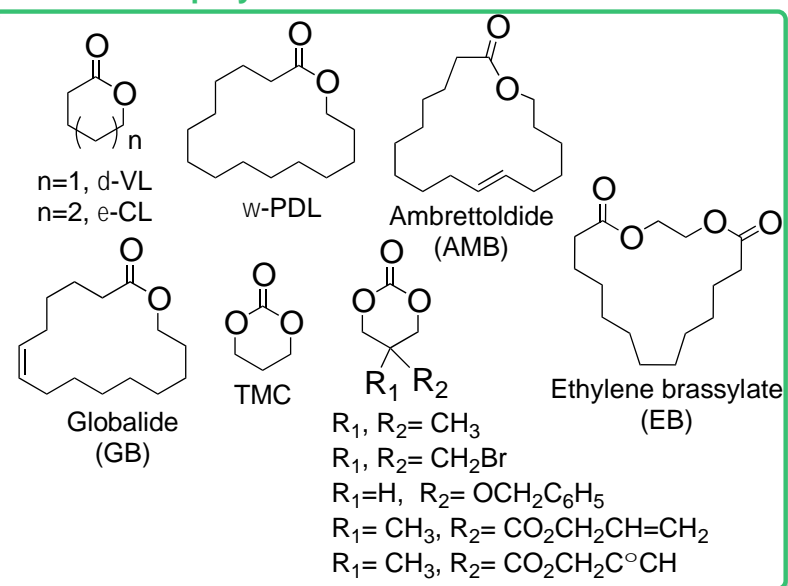

\section{Activation mode}

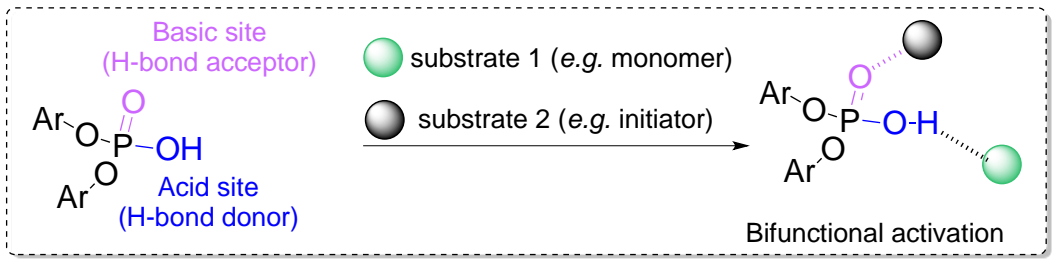

Figure 7. Representative phosphoric acids and monomers polymerized by them, as well as bifunctional activation of monomer and initiator with Brønsted acid/base pair.[43, 56]

In 2011, Kakuchi et al. described the synthesis of well-controlled PVL and PCL by ROP of $\varepsilon-\mathrm{CL}$ and $\delta$-VL in toluene at r.t., using diphenyl phosphate (DPP; 16a in Figure 7; $\mathrm{X}=\mathrm{H}$ ) as organocatalyst and 3-phenyl-1-propanol (PPA) as initiator (Scheme 19a).[57] At [ס-VL or $\varepsilon$ $\mathrm{CL}]_{0} /[\mathrm{PPA}]_{0} /[\mathrm{DPP}]$ ratio of $50 / 1 / 1$, polymerizations reached full conversion after a few hours, leading to polyesters of molar masses $\left(\mathrm{M}_{\mathrm{n}}\right)$ of 5,900 g. mol ${ }^{-1}$ and narrow dispersity $\Theta<1.09$ in both cases. PVL- $b$-PCL block copolymers were also synthesized by sequential ROP, regardless of the monomer addition sequence. Under the same conditions, bis(4-nitrophenyl) phosphate (BNPP; 16b in Figure 7; $X=\mathrm{NO}_{2}$ ), proved an efficient organocatalyst for the ROP of $\beta$-BL.[58] While DPP was inefficient in promoting the ROP, introduction of a nitro group into the DPP structure allowed enhancing the acidity, leading to PBL's of $M_{n}=2,200-10,700 \mathrm{~g} \cdot \mathrm{mol}^{-1}$ $(\Xi=1.20-1.39)$ in a controlled fashion.

a)

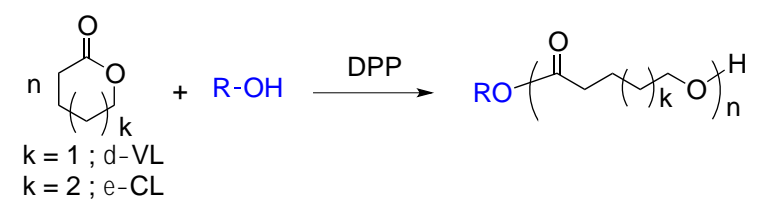

b)
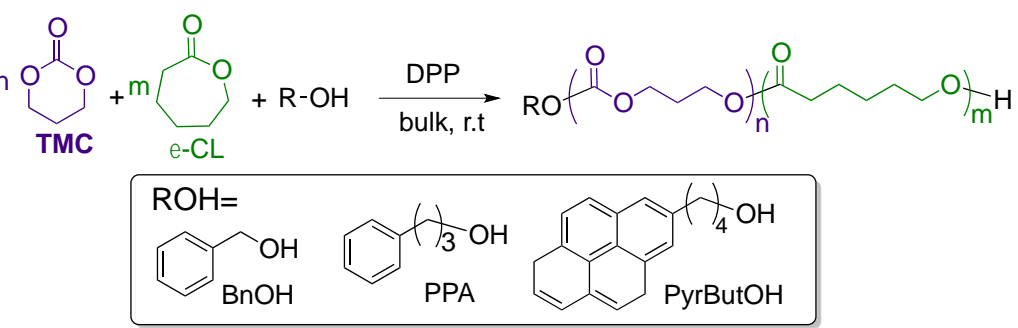

Scheme 19. ROP of $\varepsilon$-CL and $\delta$-VL using diphenyl phosphate (DPP) and alcohols $(\mathrm{ROH})$ as initiators.[57] 
The DPP/ROH catalytic system was also applied by Mecerreyes et al. for the ROP of macrolactones, $\omega$-PDL, AMB, GB and EB (see Figure 7 for related structures),[37, 38] both in bulk and in aqueous miniemulsion at $80^{\circ} \mathrm{C}$, with $\mathrm{BnOH}$ as initiator. For a DPP concentration of 0.5-10 mol\% relative to $\mathrm{BnOH}$, aliphatic polyesters of $M_{n}=5,600-21,000 \mathrm{~g} \cdot \mathrm{mol}^{-1}$, but with a rather high dispersity, $\Xi=1.65-2.53$, were obtained. The polymerization was slower than in the case of stronger acids, such as $\mathrm{TfOH}$, DBSA, or PTSA, in agreement with the lower acidity of DPP. An AMM was proposed in the case of the ROP of these macrolactones under bulk conditions.

DPP was also shown to catalyze the ROP of TMC in bulk or in dichloromethane at r.t., using PyBuOH, or PPA as initiators.[43, 56] Polymerizations reached full conversion after $22 \mathrm{~h}$, producing PTMC's of controlled molar masses $\left(M_{n}\right.$ up to $\left.11,000 \mathrm{~g} \cdot \mathrm{mol}^{-1} ; \bigoplus=1.06\right)$, in accordance with the initial monomer-to-initiator ratio.

In the same way, Kakuchi et al. reported the ROP of cyclic carbonates possessing functional groups, such as, 5,5-dimethyl-1,3-dioxan-2-one, 5,5-dibromomethyl-1,3-dioxan-2one, 5-benzyloxy-1,3-dioxan-2-one, 5-methyl-5-allyloxycarbonyl-1,3-dioxan-2-one, and 5methyl-5-propargyloxycarbonyl-1,3-dioxan-2-one (Figure 7), with PPA as initiator and DPP as catalyst.[56] Diblock copolymers consisting of a PTMC segment and a second PCL or PVL block were obtained in bulk at r.t., with molar masses increasing from 4,900 ( $(=1.11)$ to $10,500 \mathrm{~g} \cdot \mathrm{mol}^{-1}(\Theta=1.12)$ for the TMC/E-CL system, and from $5,000(\Theta=1.09)$ to $9,800 \mathrm{~g} \cdot \mathrm{mol}^{-1}$ $(\Xi=1.14)$ for the $T M C / \delta$-VL one (Scheme $19 b)$.

Surprisingly, in contrast to TfOH (vide supra), DPP did not allow the ROP of MTC-OC $6 \mathrm{~F}_{5}$ using alcohols as initiators. Given the efficiency of DPP to polymerize TMC, this inactivity towards $\mathrm{MTC}-\mathrm{OC}_{6} \mathrm{~F}_{5}$ was ascribed to the occurrence of side reactions between the activated ester moiety and DPP. [45]

Binaphthol (BINOL)-derived monophosphoric acids (17, Figure 7) represent a special class of chiral Brønsted acid catalysts widely used for enantioselective organic transformations that have been recently applied to polymer synthesis.[59, 60] In particular, the BINOL-derived phosphoric acid, namely, binaphthyl-diyl hydrogen phosphate (17 in Figure 7 with $X=H$ ), was shown to efficiently catalyze the ROP of $\varepsilon-\mathrm{CL}$ and $\delta$-VL in bulk at $40-60{ }^{\circ} \mathrm{C}$, with $\mathrm{BnOH}$ as initiator,[61] yielding polymers with $M_{n}=4,500-12,000 \mathrm{~g} \cdot \mathrm{mol}^{-1}(\Theta=1.08-1.17)$. Interestingly, the use of selectively protected monosaccharides (mono- and polyols D-glucose derivatives) as initiators led to functionalized-carbohydrate polyesters.[62]

The axial chirality displayed by those catalysts has recently enabled the enantiomerselective polymerization of rac-LA using 3-phenyl-1-propanol (PPA) as initiator in toluene at $75^{\circ} \mathrm{C}$.[63] Depending on the nature of the 3,3'-flanking substituents on the catalysts, a maximum selectivity factor $\left(\mathrm{k}_{\mathrm{D}} / \mathrm{k}_{\mathrm{L}}\right)$ of 28.3 could be obtained at $49 \%$ of $\mathrm{LA}$ conversion $\left(M_{n}=3,700 \mathrm{~g} \cdot \mathrm{mol}^{-1} ; \Xi=1.13\right)$. From a mechanism point of view, a dual activation of the 
monomer and chain-end by the bifunctional catalyst was proposed to contribute to the high $\mathrm{k}_{\mathrm{D}} / \mathrm{k}_{\mathrm{L}}$ observed.[63]

Besides phosphoric acid catalysts, imidodiphosphoric acid (IDPA, 18 in Figure 7) have been employed for the ROP of $\delta-\mathrm{VL}$ and $\varepsilon$ - $\mathrm{CL}$ in toluene at r.t., with $\mathrm{BnOH}$ as initiator.[64] Well-

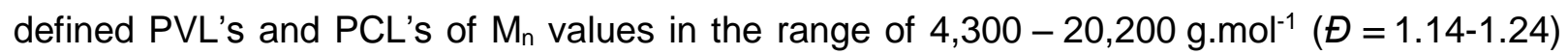
were thus obtained. IDPA was also studied for the $\mathrm{BnOH}$-initiated ROP of TMC in toluene at r.t and $50{ }^{\circ} \mathrm{C}$.[65] No side decarboxylation was noted at $50^{\circ} \mathrm{C}$, giving rise to well-defined PTMC's, with $M_{n}=1,800-18,000$ g.mol ${ }^{-1}$ and $\Theta=1.12-1.19$. Additionally, telechelic and star-shaped PTMC's were synthesized using various initiators. Well-defined PTMC- $b$-PVL and PTMC- $b$-PCL diblock copolymers could also be obtained by sequential polymerization of TMC with $\delta-V L$ and $\varepsilon-C L$, using IDPA as catalytic system.

In summary, organic acids, from weak to strong acids, have (re)emerged as a novel class of organic catalysts for polymerization reactions. In some cases, the catalyst efficiency does not simply correlate to its acidity, suggesting a different mechanism such as a dual activation of both the monomer and the initiator. Such an acidic organocatalysis proves efficient not only for the ROP of cyclic esters and carbonates, but also for the GTP of (meth)acrylics and for polyurethane synthesis.

\section{Polymerization catalyzed by phosphorous-containing Brønsted and Lewis bases: phosphazenes and phosphines}

Phosphazenes and phosphines are basic compounds (Figure 8), which have been investigated as catalysts mainly for the ROP of cyclic monomers, but also for the GTP polymerizations of (meth)acrylic substrates (Figure 9).

Phosphazenes and phosphines

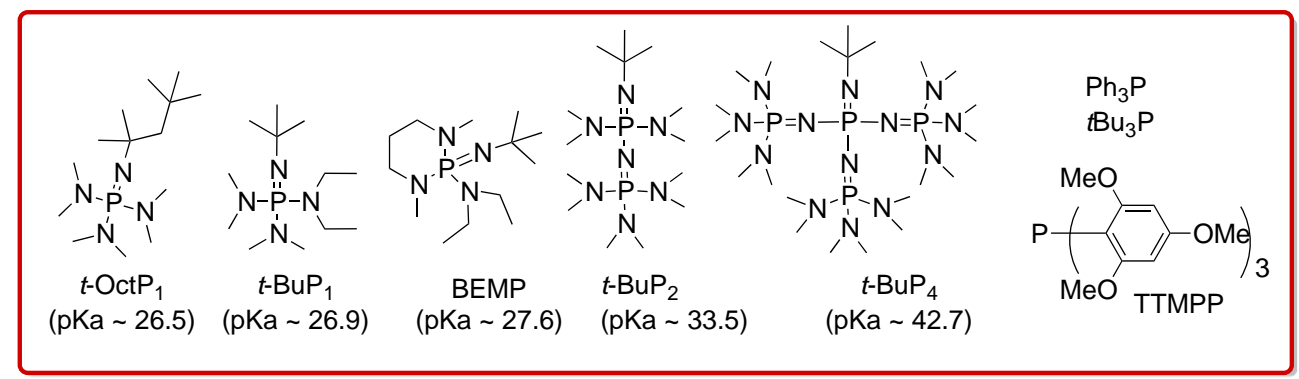

Figure 8. Representative examples of phosphazene bases (pKa values in MeCN [66]), and phosphines. 


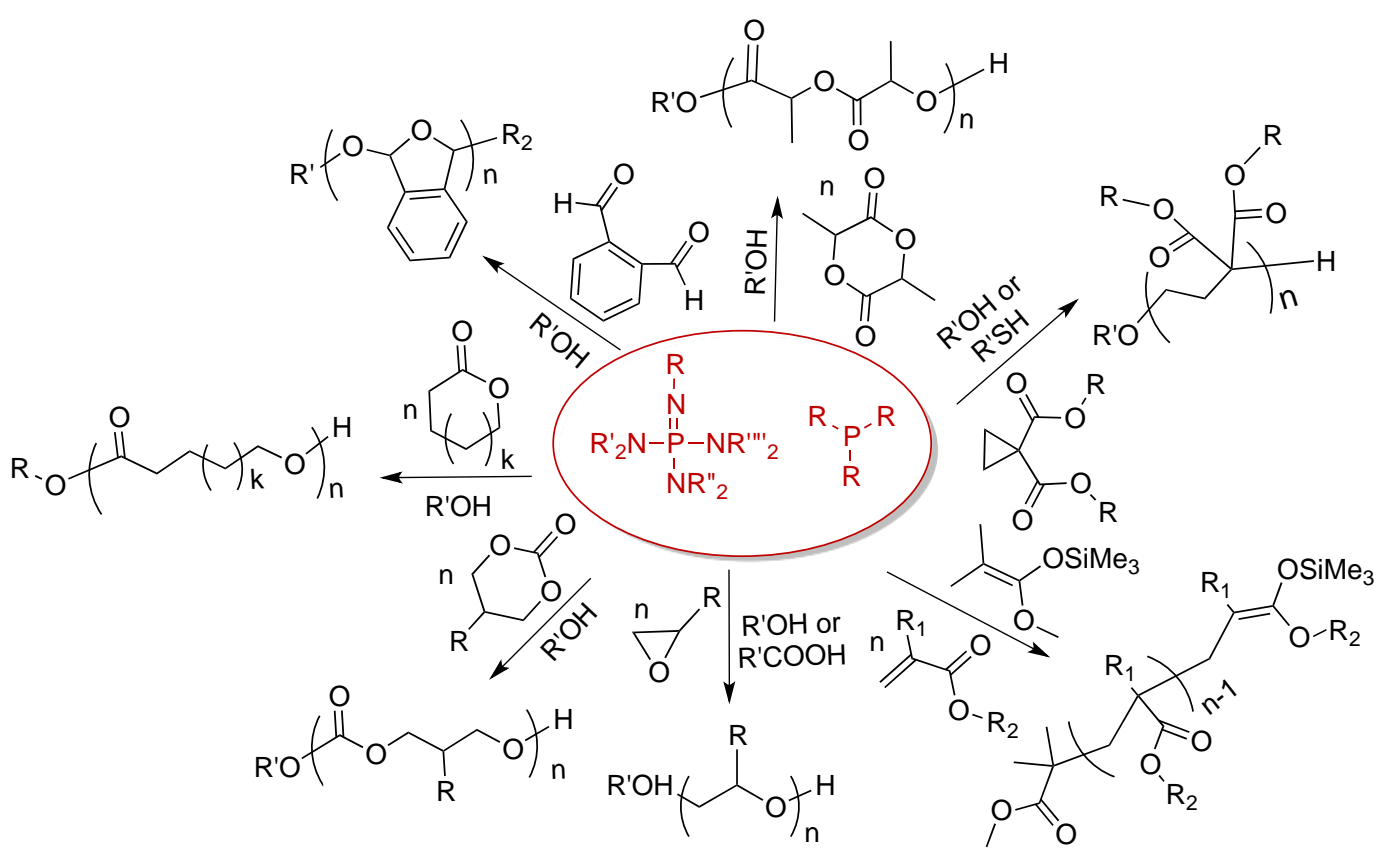

Figure 9. Overview of polymerization reactions catalyzed by phosphazenes (mainly) and phosphines.

\subsection{Phosphazenes}

Initially developed by Schwesinger,[67] phosphazenes behave as strong neutral Brønsted bases ( $\mathrm{pKa}=26-47$ in $\mathrm{MeCN}$ )[66] and are weak nucleophiles. In the context of polymer synthesis, they have been used to catalyze the ROP of cyclopropanes and heterocyclic monomers (e.g. esters, ethers or carbonates), and to promote the anionic polymerization of vinyl monomers (e.g. alkyl (meth)acrylates) as well.[68] In the presence of protic initiators, phosphazenes operate via an ACEM (see Scheme 4a), in agreement with their high Brønsted basicity and low nucleophilicity. The deprotonation of a protic alcohol initiator results in the formation of an alkoxide associated with a soft and bulky phosphazenium cation, which allows the metal-free polymerization to be controlled.[68] Other initiating systems based on thiol-, amide-, or even some $\mathrm{CH}$-containing precursors can also be used. Most of phosphazenes used in this context and monomers investigated are shown in Figures 8 and 9.

The ROP of cyclic ethers with phosphazenes as catalysts have been (re)investigated by Hadjichristidis et al. For instance, the 1-tert-butyl-4,4,4-tris(dimethylamino)-2,2bis[tris(dimethylamino) phosphoranylidenamino]- $2^{5}, 4^{5}$-catenadi(phosphazene), denoted as $t$ - $\mathrm{BuP}_{4}$ (see Figure 8 ) was employed (1.15 eq.) to trigger the ROP of several epoxides, including ethylene oxide (EO), propylene oxide (PO), 1,2-butylene oxide (BO) and tert-butyl glycidyl ether (tBGE), using poly( $N, N$-dimethylacrylamide-co-acrylamide) (CoAm) as a macroinitiator (Scheme 20).[69] In contrast to EO and PO, BO and tBGE could not undergo direct anionic ringopening graft polymerization (AROGP) from the CoAm precursor, most probably to due a steric hindrance. In toluene, at $45^{\circ} \mathrm{C}$, these polymerizations yielded macromolecular combs with various side chains, including single- or double grafted homopolymers, block and statistical 
copolymers $\left(\mathrm{M}_{\mathrm{n}}\right.$ in the range of $\left.600-1,700 \mathrm{~kg} \cdot \mathrm{mol}^{-1} ; \bigoplus=1.17-1.31\right)$. Initiation was proposed to occur through deprotonation of pendant primary amides by $t$-BuP 4 , followed by ring-opening of the monomer.

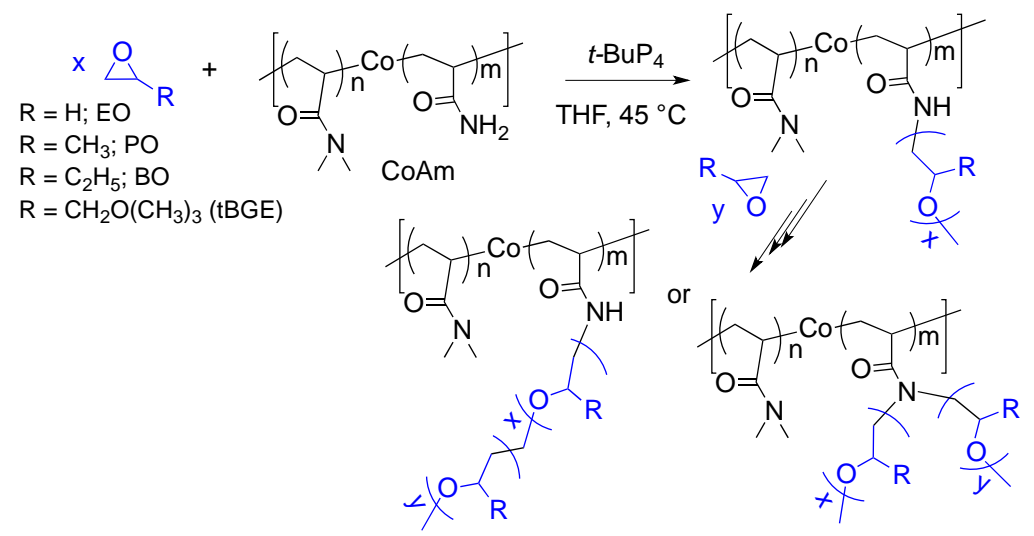

Scheme 20. $t$-BuP 4 promoted anionic graft polymerization of epoxides from acrylamidebased backbone.[69]

The polymerization of EO initiated by carboxylic compounds i.e. 1-pyrenebutyric acid (PyBA) and palmitic acid (PmA), in presence of $t-\mathrm{BuP}_{4}$ as catalyst $(10-50 \mathrm{~mol} \%$ relative to the initiator) was also investigated in THF at $45^{\circ} \mathrm{C}$ (Scheme 21).[70] PEO's thus obtained exhibited molar masses $M_{n}=1,700-2,300 \mathrm{~g} \mathrm{~mol}^{-1}$ and dispersity values in the range 1.04-1.13. Kinetic studies revealed an induction period likely resulting from the rather slow ring-opening of EO by the phosphazenium carboxylate (Scheme 22a). The resulting propagating alkoxide allowed the polymer chain to slowly grow with simultaneous fast end-group transesterification and proton transfer (Scheme 22b).

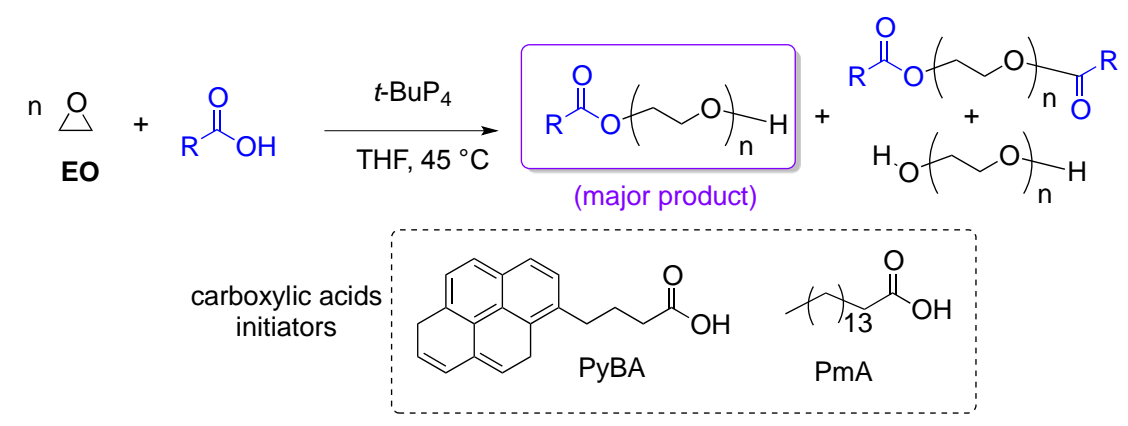

Scheme 21. $t$-BuP 4 promoted-ROP of ethylene oxide (EO) initiated by carboxylic acids.[70] 
a) Induction period (transformation of $\mathrm{COOH}$ to $\mathrm{OH}^{-}$and $\mathrm{O}^{-}$)

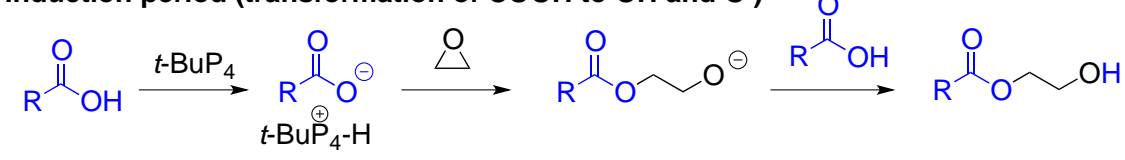

b) Chain growth (with simulataneous proton transfer and transesterification)

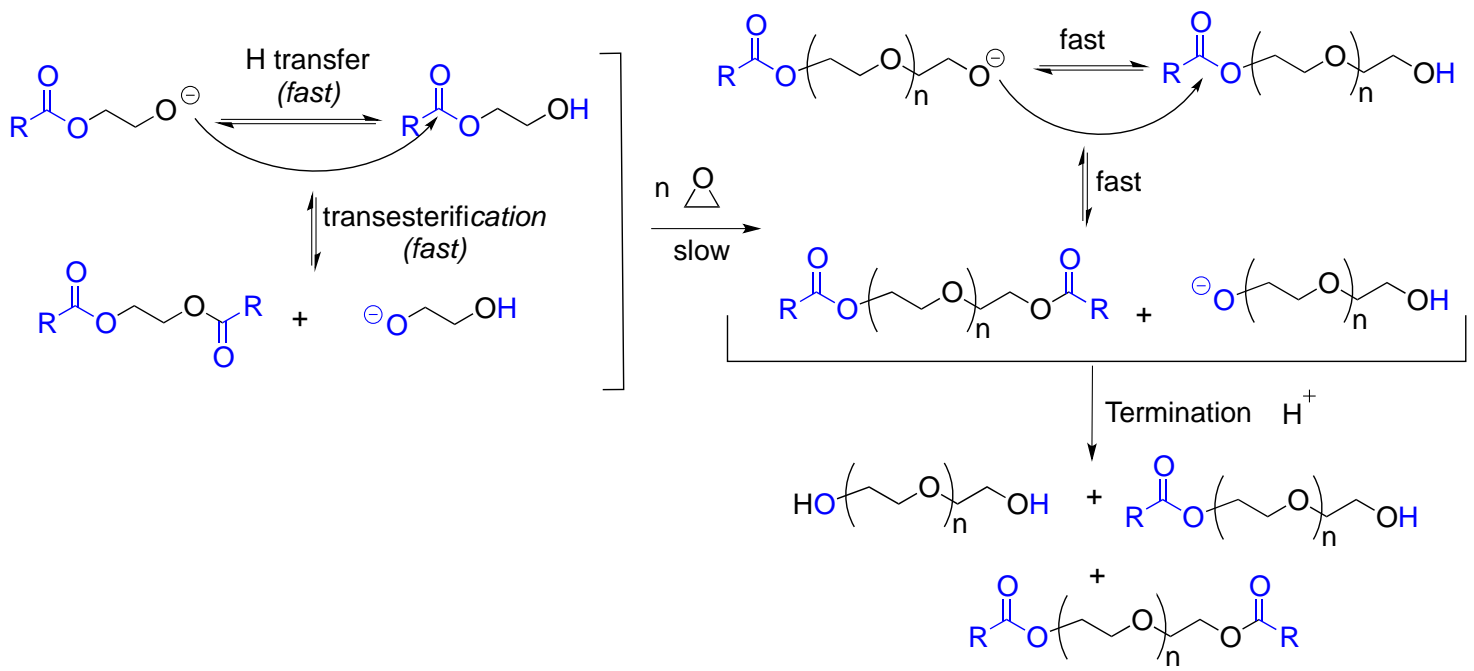

Scheme 22. Proposed mechanism for the $t$-BuP 4 promoted-ROP of ethylene oxide (EO) initiated by carboxylic acids.[70]

Sequential metal-free ROP of epoxides ( $E O$ and/or BO) and cyclic esters ( $\delta$-VL or $\varepsilon-C L$ ) or carbonates (TMC) was then reported by Hadjichristidis et al. To this end, the authors switched the polymerization catalyst, from the basic $t$-BuP 4 (for epoxides), to the acidic DPP (for cyclic esters/ carbonate).[71, 72] Thus, EO and BO were first polymerized in THF at $40^{\circ} \mathrm{C}$, using PPA as initiator and $t$-BuP 4 as catalyst (0.2 eq.), giving polyethers of $M_{n}=1,300-3,800 \mathrm{~g}^{\mathrm{mol}}{ }^{-1}$ and $\Xi=1.04-1.05$. An excess of DPP (1.2 eq.) was then introduced, followed by addition of $\varepsilon-C L$, $\delta$ VL or TMC. In this way, polyether-polyester/polycarbonate block copolymers, with molar masses $M_{n}=6,200-3,800$ g.mol ${ }^{-1}$ and $\Theta=1.04-1.72$, could be readily synthesized.

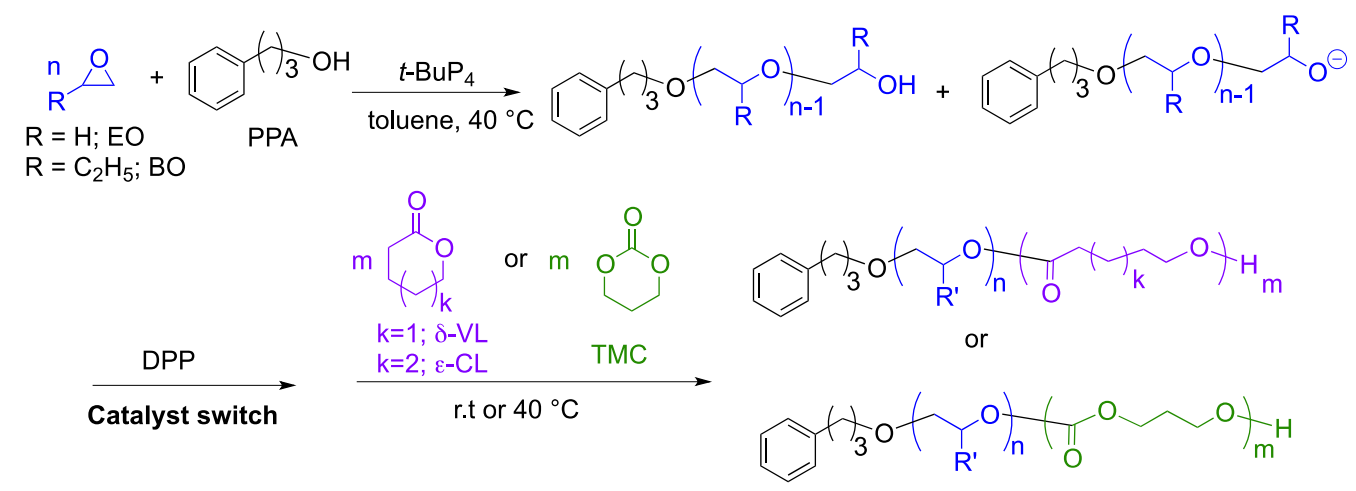

Scheme 23. Sequential ROP using a "catalyst switch" strategy, from $t-\mathrm{BuP}_{4}$ to DPP.[71, 72]

Later on, the same group studied the catalytic activity of the less-hindered phosphazene 
base, 1-tert-butyl-2,2,4,4,4-pentakis(dimethylamino)-2 $\lambda^{5}, 4 \lambda^{5}$-catenadi (phosphazene), denoted as $t$-BuP 2 (see Figure 8), for the ROP of EO, $\varepsilon-C L$ and LLA, using PPA or even water as initiators.[73] Complete EO conversion was achieved in THF after 3 days at $50{ }^{\circ} \mathrm{C}$, leading to PEO's with predictable molar mass $\left(\mathrm{M}_{\mathrm{n}}=4,600 \mathrm{~g} \mathrm{~mol}^{-1} ; \oplus=1.03\right)$. Well-defined PEO- $b$-PCL diblock and PCL- $b$-PEO- $b$-PCL triblock copolymers of $M_{n}=10,900(\Theta=1.09)$ and 13,300 g.mol ${ }^{-}$ ${ }^{1}(\Theta=1.11)$, respectively, were next generated by addition of $\varepsilon-C L$ to the PEO living chains. Pentablock copolymers, PLLA-b-PCL-b-PEO-b-PCL- $b$-PLLA $\left(\mathrm{M}_{\mathrm{n}}=16,100 \mathrm{~g} \cdot \mathrm{mol}^{-1} ; \quad \bigoplus=1.11\right)$, were even synthesized by introduction of LLA (nearby complete conversion of LLA within $10 \mathrm{~min}$ ). It was however essential to follow the EO/ $\varepsilon$-CL/L-LA addition in order to avoid any interor intra-macromolecular side reactions, which could occur during the ROP of EO with PCL or PLLA macroinitiators. The same group investigated the influence of solvents over such $t$ $\mathrm{BuP}_{2}$-catalyzed ROP of $\varepsilon$-CL and L-LA at r.t., using primary alcohols as initiators. [74] While a higher polymerization rate was noted in more polar solvents (dichloromethane vs. toluene), slower polymerization reactions were noted in cyclic ether solvents (THF, 1,4-dioxane vs. toluene). Due to their slight basicity, these solvents might indeed interact with initiating species, thus slowing down the ROP.

Phosphazene catalysts were also applied to the ROP of cyclic carbonates. For instance, Guillaume, Carpentier et al. reported the one-pot, two-step sequential copolymerization of TMC and L-LA in toluene at $100{ }^{\circ} \mathrm{C}$, using the phosphazene 2-tert-butylimino-2-diethylamino-1,3dimethylperhydro-1,3,2-diazaphosphorine (BEMP see Figure 8; 0.02 eq.) combined with $\mathrm{BnOH}$ as initiator.[75] TMC was first polymerized prior to the addition of L-LA, yielding PTMC- $b$-PLLA diblock copolymers of molar masses $M_{n}=36,200 \mathrm{~g} \cdot \mathrm{mol}^{-1}(\theta=1.32)$. While PLLA is a tough and brittle material, PTMC- $b$-PLLA block copolymers demonstrated better thermo-mechanical properties, evidencing the advantage of the presence of a rubbery PTMC sequence.

In the same way, tert-octylimino-tris(dimethylamino)phosphorane $\left(t-\mathrm{OctP}_{1}\right), t-\mathrm{BuP}_{2}$, and $t$-BuP 4 -catalyzed ROP of TMC, in the presence of naturally occurring native starch granules as macroinitiators, proceeded in bulk at $150{ }^{\circ} \mathrm{C}$ (Scheme 24).[76] This strategy allowed synthesizing polycarbonate/starch composite materials with enhanced physical and biodegradability properties over the native starch. The phosphazene catalysts showed an excellent catalytic activity ( $98 \%$ of monomer conversion after only $2 \mathrm{~min}$ of reaction), even at very low concentration (e.g. with 0,01 mol\% of $t$-BuP 4 ). PTMC's with moderate molar masses $\left(M_{n}=3,800-5,200 \mathrm{~g} \mathrm{~mol}^{-1} ; \bigoplus=1.24-1.34\right)$ and surface modified starch-graft-PTMC having a $\mathrm{DP}=2-12$ (depending on the measurement technique) were thus obtained, demonstrating the robustness of phosphazenes toward the presence of starch and water. The successful homopolymerization of TMC was in fact explained by the presence of propan-1,3-diol formed by TMC hydrolysis and playing the role of a co-initiator. It can be hypothesized that this hydrolysis might also reduce the amount of water in the system, explaining the activity of those strong bases even at low concentrations. 


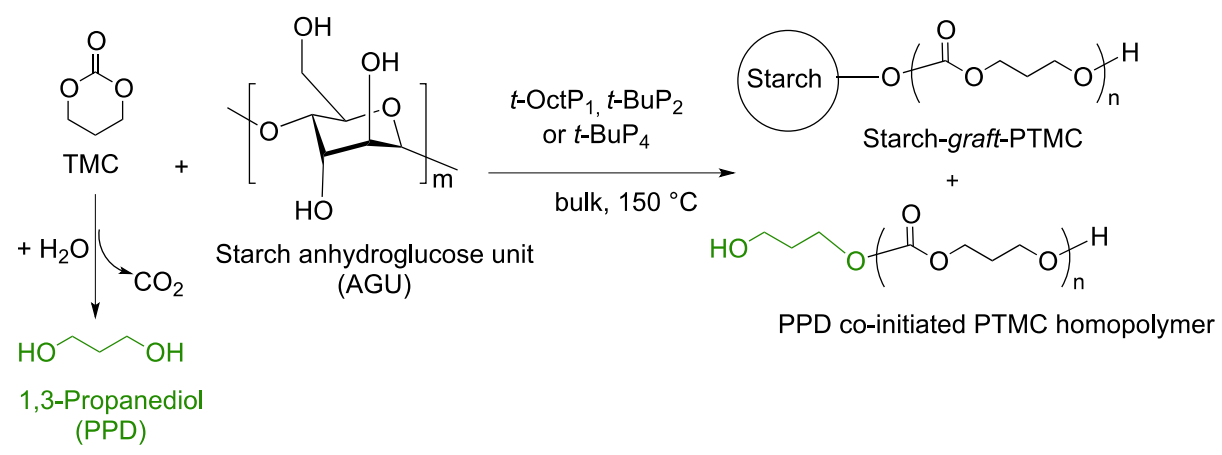

Scheme 24. Sequential ROP of TMC in the presence of native starch granules (anhydroglucose unit) using phosphazenes as catalysts.[76]

Penelle, Barbier, Boileau et al. developed the organo-catalyzed ROP of specific cyclopropane monomers, namely, dialkyl cyclopropane-1,1-dicarboxylates. For instance, the polymerization of di-n-allyl cyclopropane-1,1-dicarboxylate (DACDC) was triggered by $t$-BuP $\mathrm{B}_{4}$ as catalyst (1eq.), in presence of thiophenol as initiator (Scheme 25a).[77] Quantitative conversions were obtained after 2 days in THF at r.t., and final polymers exhibited experimental molar masses close to theoretical ones $\left(M_{n}=3,900-12,500 \mathrm{~g} \cdot \mathrm{mol}^{-1} ; \triangleq=1.1-1.2\right)$. Poly(DACDC) chains were endowed of pendant geminated allyl groups, as expected. The density of allyl groups along the polymer backbone could be further modulated through copolymerization experiments with di- $n$-propyl cyclopropane-1,1-dicarboxylate (DPCDC; Scheme 25b). Statistical and block copolymers were obtained and post-polymerization modification could be readily achieved by thiol-ene chemistry.

a)

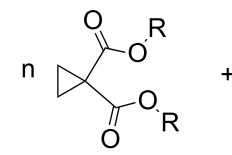

$\mathrm{R}=\left(\mathrm{C}_{3} \mathrm{H}_{6}\right)_{2} ; \mathrm{DPCDC}$

$\mathrm{R}=\mathrm{CH}_{2} \mathrm{CHCH}_{2}$; DACDC

b)

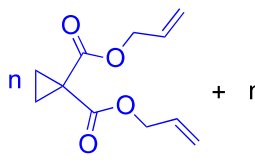

DACDC<smiles>Sc1ccccc1</smiles>

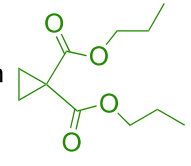

DPCDC

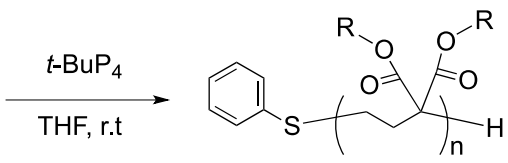

poly (DPCDC) or poly (DACDC)
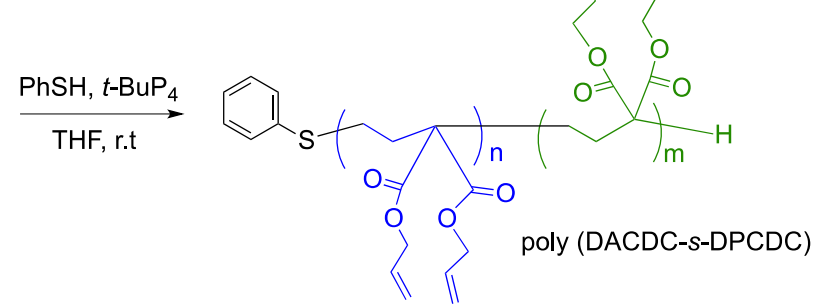

Scheme 25. ROP of cyclopropane carboxylates (DPCPC and DACPC) in the presence of $\mathrm{PhSH}$ as initiator and $t$-BuP 4 as catalyst: a) homopolymerization; b) statistical copolymerization.[77]

Phosphazenes also enabled directly polymerizing methacrylic monomers. Zhang et al. thus reported that $t$-BuP 4 could induce the copolymerization of $\varepsilon$-CL with methyl methacrylate (MMA), tert-butyl methacrylate (tBuMA) or glycidyl methacrylate (GMA) in toluene at r.t., in presence of various initiators, i.e., $\mathrm{MeOH}$, ethylene glycol (EG) or ethyl acetate (EA) (Scheme 26). [78-80] According to NMR analyses and thermal characterizations, random copolymers, 
PCL-co-PMMA or PCL-co-PtBuMA, were obtained with molar masses $\left(\mathrm{M}_{\mathrm{n}}\right)$ in the range

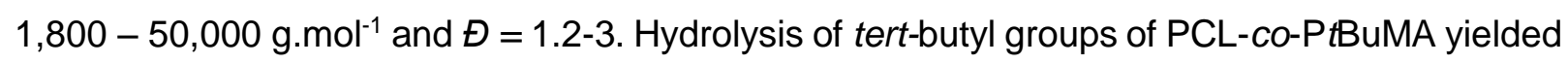
$\operatorname{poly}(\varepsilon-C L-c o-m e t h a c r y l i c ~ a c i d)$, poly(CL-co-MAA), i.e. a degradable statistical copolyelectrolyte. The authors suggested that copolymerization occurred via a hybrid mechanism, involving a combination of vinyl polymerization and ROP through alcohol activation by $t$-BuP 4 . The activated alcohol would attack the monomer ( $\varepsilon-C L$ or MMA, with $\varepsilon-C L$ being the most reactive), producing two kinds of active centers, 19 and $\mathbf{2 0}$ (of alkoxide- and enolate-type, respectively, Scheme 26). These active centers would then be able to react with either MMA or $\varepsilon-C L$, forming new propagating centers. However, the reaction mechanism was not fully elucidated, and some side reactions such as homopolymerization or transesterification reactions might have occurred.

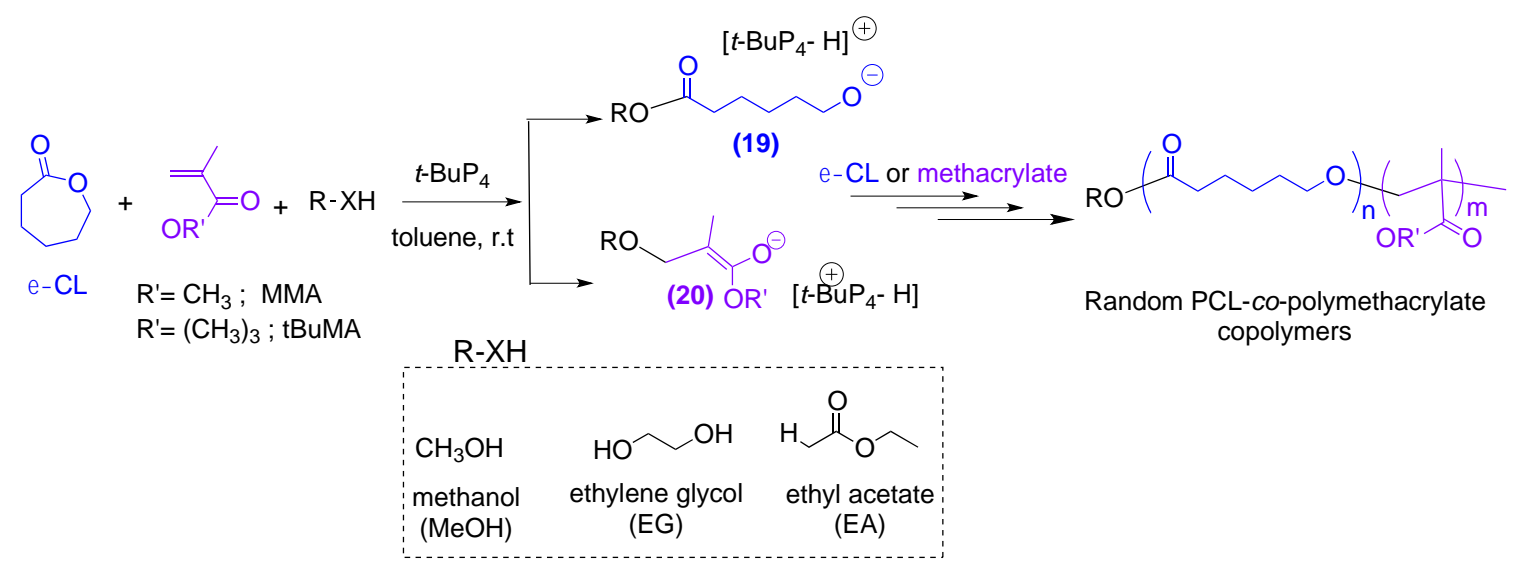

Scheme 26. Hybrid copolymerization of cyclic esters $\varepsilon-C L(R O P)$ with methacrylic monomers MMA and tBuMA (1,4-conjugate addition polymerization). [78-80]

Recently, Coulembier et al. investigated the use of $t-\mathrm{BuP}_{1}$ as catalyst in the cyclopolymerization of ortho-phthalaldehyde (PA) initiated by either 9-anthracenemethanol or MeOPEO-OH in dichloromethane at $-85^{\circ} \mathrm{C}$ (Scheme 26).[81] Poly(ortho-phthalaldehyde)'s (PPA's) are attractive materials due to their spontaneous depolymerization in response to a radiation stimulus. $[82,83]$ Using the $t$-BuP $_{1}$-catalysis, PPA's and PEO-b-PPA diblock copolymers of molar masses $M_{n}=3,200-200,000 \mathrm{~g} \mathrm{~mol}^{-1}(\Theta=1.15-1.27$ ) were synthesized with high end-group fidelity.

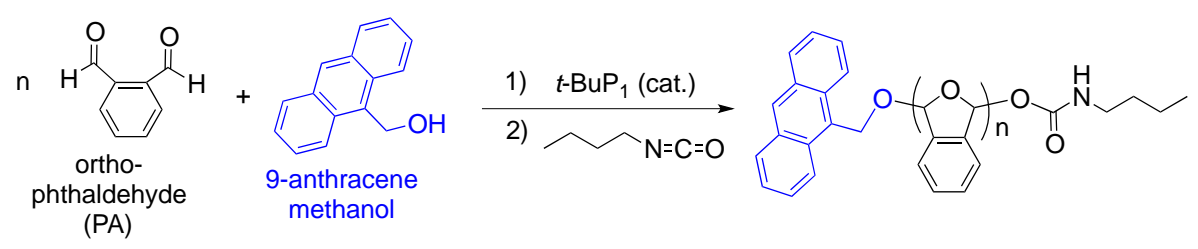

Scheme 27. tBu-P1-catalyzed cyclopolymerization of ortho-phthalaldehyde.[81]

\subsection{Phosphines}

Even though phosphines have been widely employed as catalysts in a variety of organic transformations,[84, 85] their use in polymerizations reactions has been scarcely investigated.[86] Alkyl/aryl phosphines such as tributylphopshines $\left(\mathrm{Bu}_{3} \mathrm{P}\right)$, 
dimethylphenylphosphine $\quad\left(\mathrm{PhMe}_{2} \mathrm{P}\right), \quad$ methyldiphenylphosphine $\quad\left(\mathrm{Ph}_{2} \mathrm{MeP}\right)$ and triphenylphosphine $\left(\mathrm{Ph}_{3} \mathrm{P}\right)$, were shown to be effective catalysts for the ROP of $L A$, generating narrowly dispersed polylactides with predictable molar masses $\left(M_{n}=2,800-13,000 ; \emptyset=1\right.$. 11 1.40) through a nucleophilic AMM (Scheme 2).[86] The substitution pattern of the phosphine was a dominant feature in controlling their reactivity. Thus, alkyl-substituted phosphines, being the most basic and most nucleophilic phosphines, proved more active than their aryl-containing counterparts.[86]

In 2012, Taton et al. reported that tris(2,4,6-trimethoxyphenyl)phosphine (TTMPP; see Figure 8) could efficiently catalyze the GTP of MMA and tert-butyl acrylate (tBuA), using 1methoxy-2-methyl-1-[(trimethylsilyl)oxy]prop-1-ene (MTS) as initiator (Scheme 28).[87] GTP experiments could be conducted in bulk or in THF at r.t. This TTMPP-catalyzed GTP provided poly[alkyl(meth)acrylate]s of $M_{n}=3,000-10,600$ g.mol ${ }^{-1}(\Xi<1.37$ in bulk and $\boxminus<1.45$ in THF), in accordance with the initial [monomer $]_{0} /[\mathrm{MTS}]_{0}$ ratio. Through sequential polymerization, PMMA-b-PtBuA diblock copolymers of controlled molar masses $\left(M_{n}=22,600 \mathrm{~g} \cdot \mathrm{mol}^{-1} ; \boxminus=1.19\right)$ were also synthesized. TTMPP-derived PMMA's exhibited a tacticity of $\mathrm{mm} / \mathrm{mr} / \mathrm{rr}$ $=0.06 / 0.41 / 0.53$, i.e. very similar to that of PMMA grown by an anionic process, suggesting the occurrence of a dissociative mechanism (Scheme 4b), though, attempts to identify enolate-type species by NMR analysis were unsuccessful.

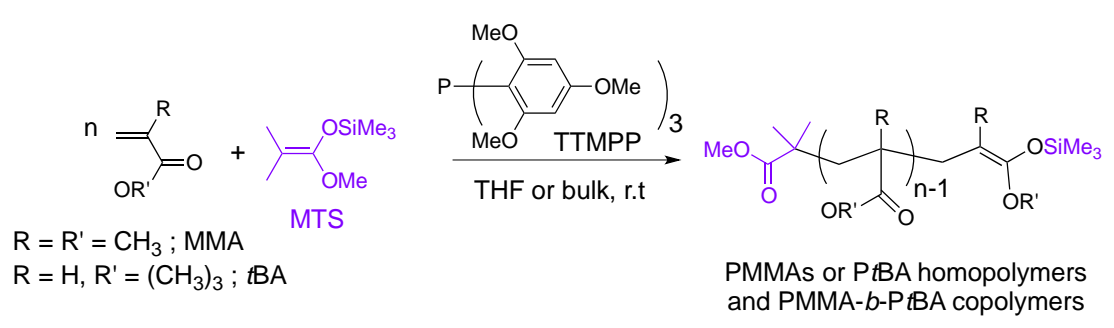

Scheme 28. Phosphine-catalyzed GTP of MMA and tBuA.[87]

In summary, commercially available phosphazene Brønsted "superbases" are powerful organocatalysts which have been mainly investigated for ROP of epoxides, cyclic esters, cyclic carbonates and cyclopropane-1,1-dicarboxlates, and few examples of polymerization of (meth)acrylics. Due to their strong basicity and low nucleophilicity, phosphazenes allowed for an in situ generation of anionic initiators by deprotonation of compounds containing acidic protons $(\mathrm{C}-\mathrm{H}, \mathrm{O}-\mathrm{H}, \mathrm{COO}-\mathrm{H}, \mathrm{S}-\mathrm{H}$ and $\mathrm{N}-H)$. Polymers with a high end-group fidelity are generally produced because of the pairing of the active anionic propagating species with the bulky and soft phosphazenium cation deriving from the phosphazene catalyst. On the other hand, despite their widespread use in molecular chemistry, phosphine Lewis bases have been underexploited as catalysts in polymerization reactions; yet, they hold great promise given the variety of structures accessible and the possibility to introduce chirality. 


\section{Polymerization catalyzed by free and latent N-heterocyclic carbenes (NHCs)}

$\mathrm{N}$-heterocyclic carbenes (NHCs) have gained increased popularity over the past 20 years. These neutral divalent species of carbon possess only six-electron valency (four electrons are involved in $\sigma$-bonds and two remained at the central carbon) and are strongly stabilized by interaction of the two nitrogen lone pairs with the empty $p$ orbital of the carbene center.[88-93]

While $\sigma$-donor properties of NHCs lead to either a Brønsted basic (pKa 15-30 in $\mathrm{H}_{2} \mathrm{O}$ )[94, 95] or a nucleophilic-type catalysis, the combination of their $\sigma$-donor and $\pi$-acceptor properties (Figure 10) has allowed the activation of various unsaturated substrates by inversion of polarity.[3, 96-100] With these properties, not only can NHCs serve as powerful ligands for transition metals,[101-103] but they have also emerged as true organic catalysts in molecular chemistry (e.g. benzoin condensation, Stetter reaction, transesterification, etc.).[104-109]

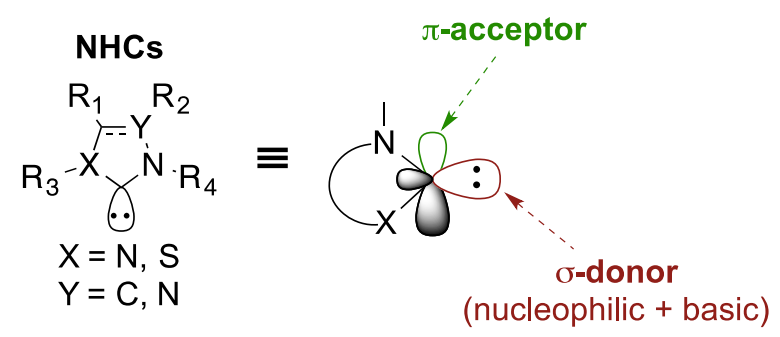

Figure 10. Electronic properties of $\mathrm{N}$-heterocyclic carbenes (NHCs).[105] scheme modified: bond added on $\mathrm{N}$ atom

In the context of macromolecular chemistry, NHCs have been applied for both chaingrowth and step-growth polymerization reactions (Figure 11) in the past decade.[7, 15, 16, 110] The field of NHC-mediated polymerization reactions has been reviewed in 2013 by our group [15] and by Naumann and Dove in early 2015.[16] Main researches have been focused on the use of "free" NHCs derived from imidazol(in)ium salts, but efforts have also been (re)directed towards the design of more air-tolerant "protected" NHCs (called "masked" NHCs). These NHC precursors are indeed generally easier to handle than bare NHCs, avoiding the use of controlled inert atmospheres.[111] Figure 12 illustrates main categories of NHCs used in polymerization. 


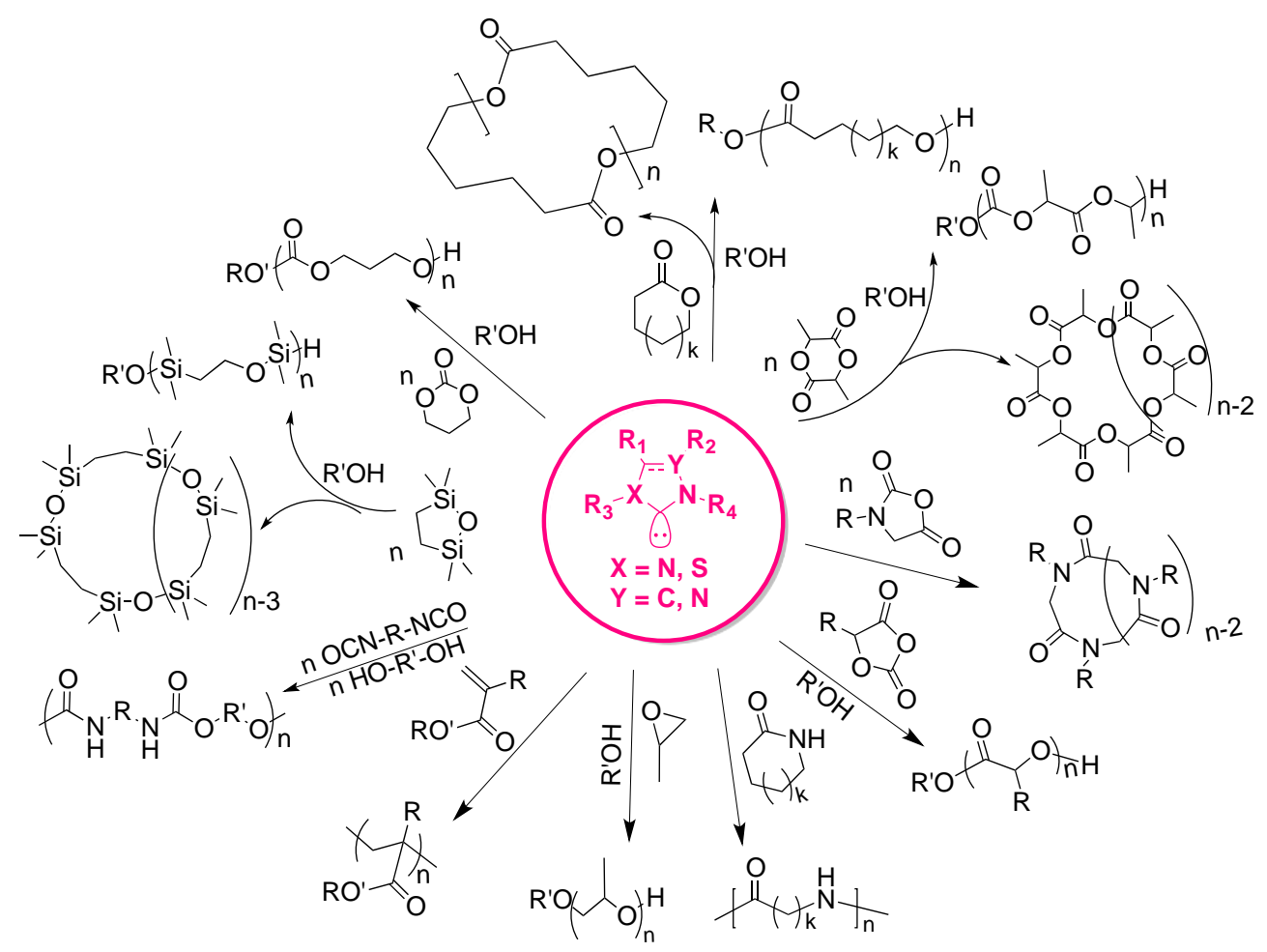

Figure 11. Scope of NHC-catalyzed polymerization reactions.

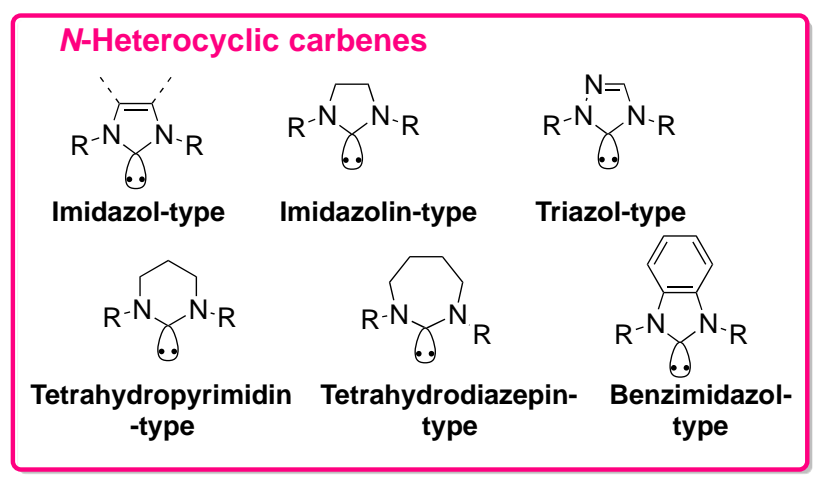

Figure 12. Representative NHC families used in polymerization reactions.

\subsection{Free NHCs}

Free NHCs are generally prepared by deprotonation of their corresponding conjugated acid imidazol(in)ium salts with a strong base, e.g. NaH / $t$-BuOK, KHMDS (route a, Scheme 29).[112] Other routes for NHC preparation include: b) electrochemical reduction of imidazol(in)ium salt,[113, 114] c) chemical reduction of imidazol-2(3H)-thiones by a metal (typically potassium)[115] or d) thermal activation of "masked" NHCs.[111] 
a) Depronation of imidazol(in)ium salts

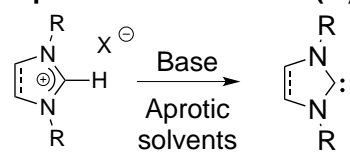

$\mathrm{X}=\mathrm{Cl}$ or $\mathrm{Br}$

Base $=$ NaH, $t$-BuOK,KHMDS, LDA, $n$-BuLi....

c) Reduction of imidazol(in)-2-thiones

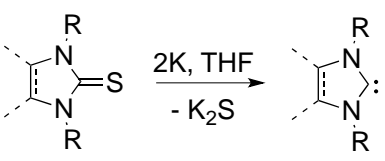

b) Electrochemical reduction of imidazol(in)ium salts

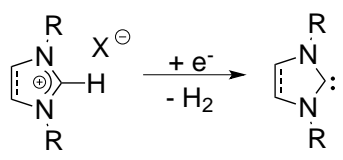

d) Thermolysis of "masked" NHCs

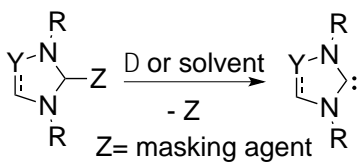

Scheme 29. Main synthetic routes for the generation of imidazole(in)-2-ylidenes. [111-115]

Hedrick, Waymouth et al. previously demonstrated that, in the presence of alcohol initiators, NHCs could efficiently catalyze the ROP of cyclic esters (lactide and lactones) with a turn over frequency (TOF) of $18 \mathrm{~s}^{-1}$, while exhibiting many features of a controlled polymerization.[7] In agreement with experimental observations, DFT calculations suggested that the polymerization operated by a combination of both a nucleophilic AMM (Scheme 3a) and a H-bonding ACEM (Scheme 4a). Competition between both mechanisms might occur, particularly at low alcohol concentration (high monomer/initiator ratios), for which experimental molar masses deviated from those predicted.[7]

The ROP of cyclic esters have been extensively described, with a variety of NHCs. For instance, a series of 1,3-non symmetrically substituted imidazol-2-ylidenes (NHC12-15; Scheme 30) have been employed as catalysts for the ROP of L-LA[116], $\varepsilon-C L$ and 2,2dimethyltrimethylene carbonate (DTC)[117], in the presence of $\mathrm{BnOH}$ as initiator (Scheme 30). Homopolymers ( $\mathrm{M}_{\mathrm{n}}$ up 26,800 g. mol ${ }^{-1} ; \bigoplus=1.3-1.5$ ) were obtained in quantitative yield, as well as PCL- $b$-PDTC diblock copolymers $\left(\mathrm{M}_{\mathrm{n}}\right.$ up $\left.28,500 \mathrm{~g}_{\mathrm{mol}}{ }^{-1} ; \bigoplus=1.4-1.5\right)$. The catalytic activities of these NHCs significantly increased with the electron-donating ability of the substituents. For instance, $\mathrm{NHC} 14$ was found more efficient than $\mathrm{NHC} 12$ and $\mathrm{NHC13}$ for controlling the ROP of $\varepsilon-C L$ and DTC.[117]

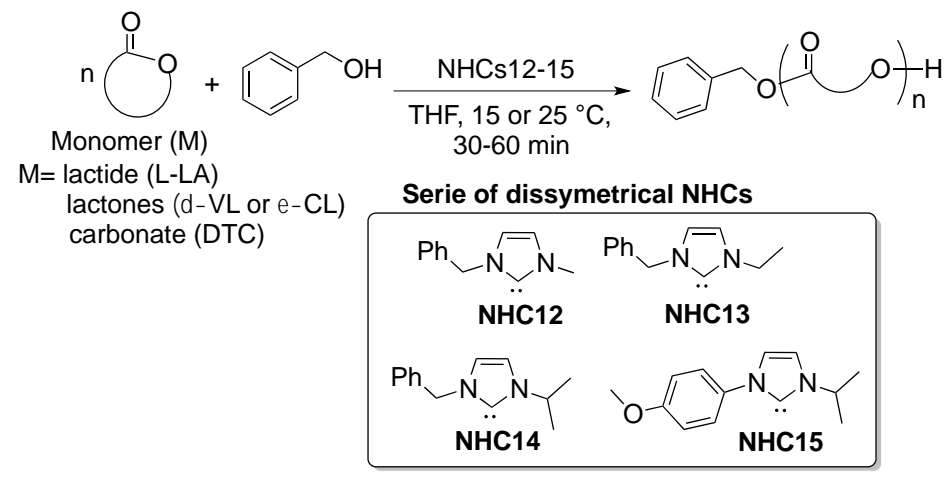

Scheme 30. ROP of cyclic esters and carbonates catalyzed by dissymmetrical NHCs in presence of $\mathrm{BnOH}$ as initiator.[116, 117]

Symmetrical imidazol-2-ylidenes (NHC1, NHC7-8; see Figure 2) and imidazolin-2- 
ylidenes (NHCs 24-25) were screened for the ROP of the O-carboxyanhydride (OCA) of L-lactic acid (L-lacOCA), using $\mathrm{BnOH}$ as initiator (Scheme 31).[118] All these carbenes allowed polymerizing L-lacOCA in THF at r.t., yielding poly(L-lacOCA) of controlled molar masses $\left(M_{n}=1,100-12,700 \mathrm{~g} \cdot \mathrm{mol}^{-1} ; \quad D=1.10-1.41\right)$. No significant differences between imidazol(unsaturated) and imidazolin-type (saturated) carbenes were noted. Within a same family, kinetics and polymerization control increased with the steric hindrance of the carbene $(\mathrm{NHC} 7>\mathrm{NHC} 8>>\mathrm{NHC} 1)$. This difference was attributed to the lower stability of the less hindered NHCs, and their tendency to dimerize, especially for NHC1.[119]

Additionally, polylol-type macroinitiators were used to derive $\alpha, \omega$-dihydroxy telechelics, star-shaped polymers and diblock copolymers based on poly( $\alpha$-hydroxy acid) (PAHA) by NHCcatalyzed ROP of L-lacOCA and L-manOCA (OCA of L-mandelic acid; Scheme 31).[118]

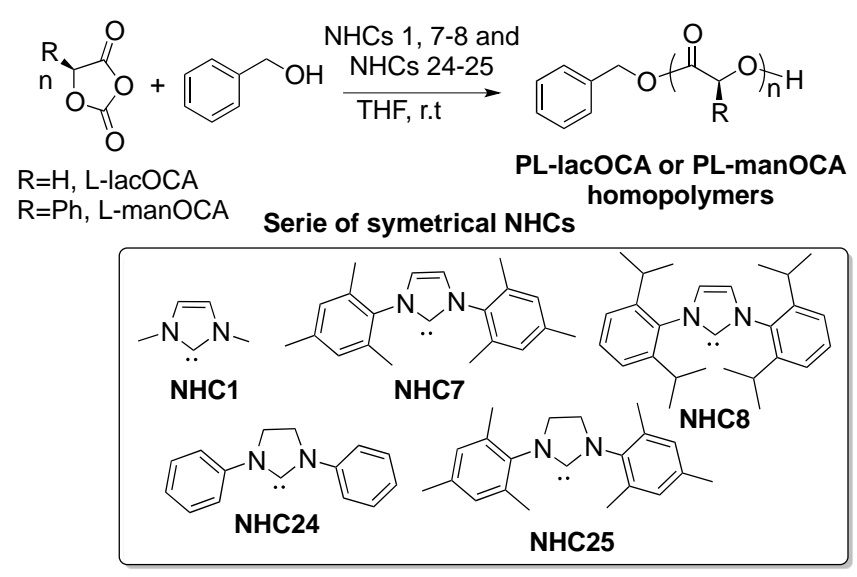

Scheme 31. BnOH-initiated ROP of L-lacOCA and L-manOCA catalyzed by NHCs.[118]

Besides chain-growth polymerizations, free NHCs were successfully used to catalyze the step-growth polymerization of diols and aliphatic diisocyanates, yielding PUs, as reported by Destarac et al.[120] For instance, the $\mathrm{NHC}^{\mathrm{tBu}}(=\mathrm{NHC})$-catalyzed polymerization between 1,4butanediol and isophorone diisocyanate (IPDI) in THF at $30-50^{\circ} \mathrm{C}$, gave soluble linear PUs with complete monomer consumption within 15 min (Scheme 32).[120] A slight increase in molar masses with time was observed, from $M_{n}=1,900 \mathrm{~g} \cdot \mathrm{mol}^{-1}$ to $2,800 \mathrm{~g} \cdot \mathrm{mol}^{-1}$, after $15 \mathrm{~min}$ and $2 \mathrm{~h}$ of polymerization, respectively. Although NHCs are capable to catalyze the di- and/or the trimerization reaction of monoisocyanate,[121] only traces of uretdione (cyclic dimer) and isocyanurate (cyclic trimer) products (Scheme 33) were detected with alkyl isocyanates, while a mixture of cyclodimer (70\%) and cyclotrimer (30\%) was obtained with phenyl isocyanate. It was postulated that the $\mathrm{NHC}$ catalyst activated the alcohol through $\mathrm{H}$-bonding (= basic mechanism), followed by a nucleophilic addition of the activated alcohol onto the isocyanate moiety (see insert in Scheme 32). 


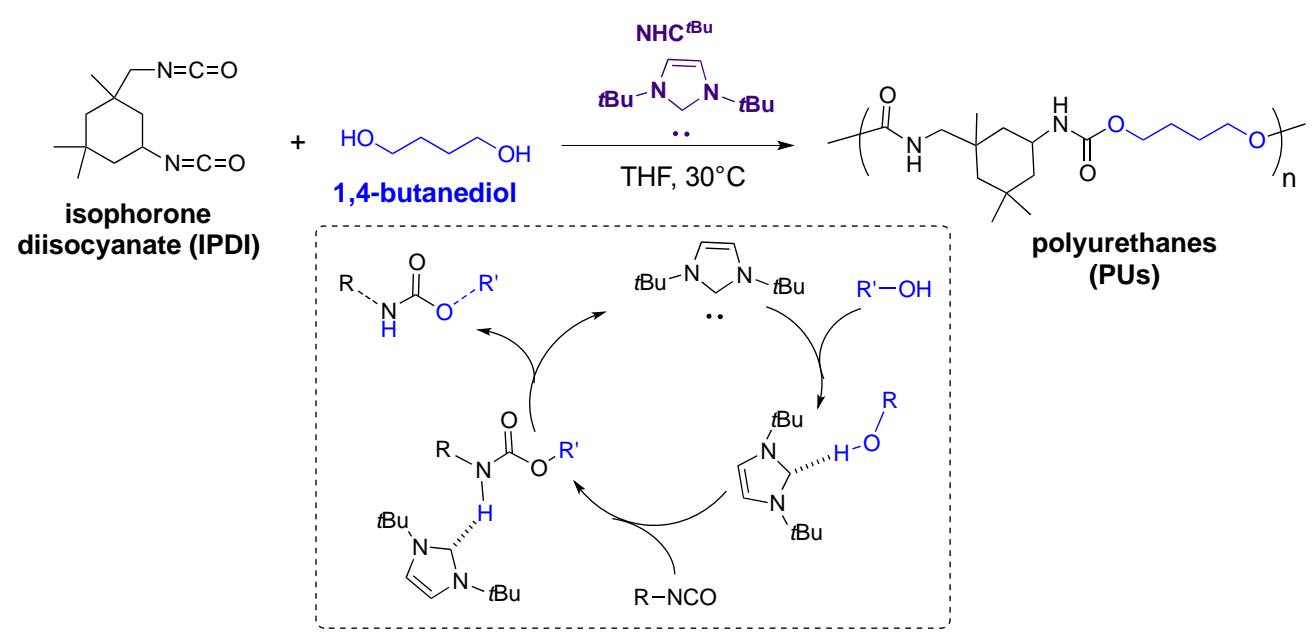

Scheme 32. NHC-catalyzed step-growth polymerization of diisocyanates with diols.[120]

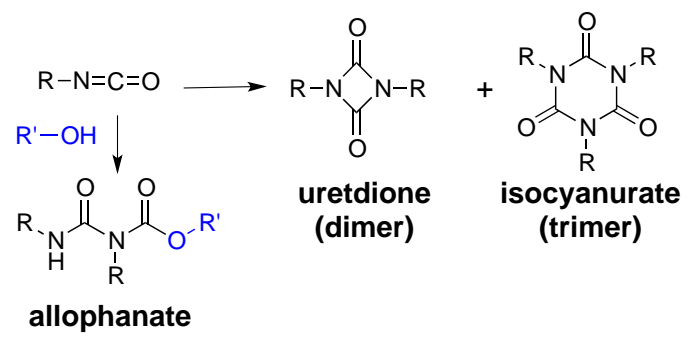

Scheme 33. Potential side reactions during polyurethane formation.[121]

Despite the efficiency of free NHCs as catalysts for polymerization reactions, their air- and moisture-sensitivity have limited their widespread adoption. Thus, recent studies have focused on the development of protected carbenes, as more air-tolerant precursors.

\subsection{Protected-NHCs}

In 2014, Buchmeiser and Naumann have published a mini-review describing pros and cons of several classes of protected-NHCs used in metal-free polymerization reactions.[111] Protected- or masked-NHCs are adducts or complexes that can release the free carbene upon application of an external stimulus, such as temperature or solvent effect.[111] Examples of these protected $\mathrm{NHCs}$ include imidazol(in)ium carboxylates $\left(\mathrm{NHC}-\mathrm{CO}_{2}\right), \mathrm{NHC}$-alcohol-adducts, some NHC-metal complexes and azolium hydrogen carbonates $\left(\left[\mathrm{NHC}(\mathrm{H}) \cdot \mathrm{HCO}_{3}\right]\right)($ Figure 13).

In this context, various protected-NHCs adducts have been designed for a use as latent catalysts in polymer synthesis. 


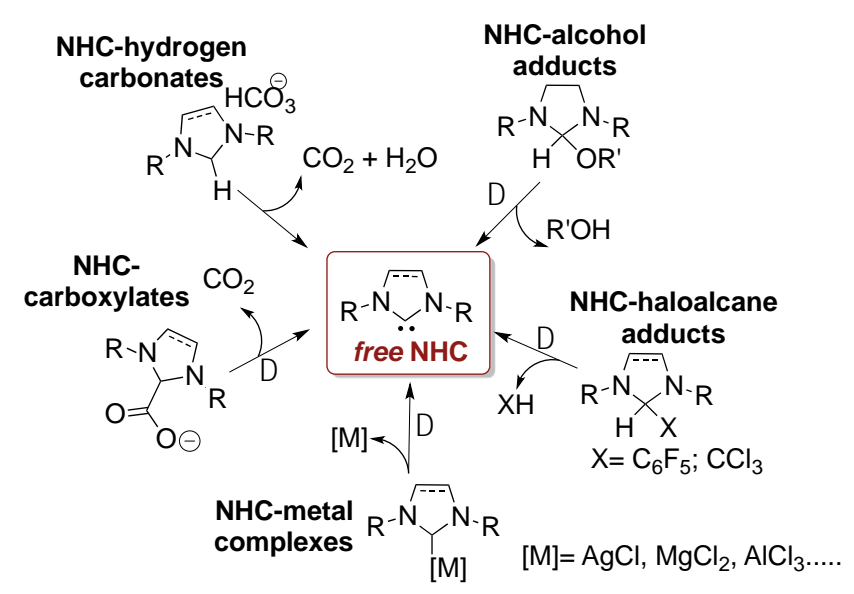

Figure 13. Thermal generation of free NHC from NHC-adducts.[111]

For instance, $\mathrm{NHC}$-carboxylate pre-catalysts ( $\mathrm{NHC}-\mathrm{CO}_{2}$ adducts) have been studied for the ROP of cyclic esters, such as lactide and lactones. Plasseraud et al. thus reported the solvent-free ROP of rac-LA, using 1-n-butyl-3-methylimidazolium-2-carboxylate (BMIM-CO ${ }_{2}$; $\mathrm{NHC} 11 ; 1 \mathrm{~mol} \%$ relative to the monomer) and alcohol initiators, such as $\mathrm{BnOH}$, ethylene glycol (EG), glycerol and pentaerythritol. Reactions were carried out in vacuo at $75^{\circ} \mathrm{C}$.[122] Conversions in the range of $50-70 \%$ were achieved, leading to PLA-based oligomers $\left(M_{n}=500-1,000 \mathrm{~g} \cdot \mathrm{mol}^{-1} ; \oplus=1.16-1.62\right)$. The incomplete conversion observed was attributed to the increase of the viscosity during PLA formation.

In contrast, the solvent-free ROP of $D, L-l a c t i d e ~(D, L-L A)$ at $r$.t and at $80^{\circ} \mathrm{C}$, in presence of $\mathrm{BnOH}$ (1 eq.) using imidazolium-2-carboxylates compounds, namely, 1,3bis(isopropyl)imidazolium carboxylate $\left(\mathrm{NHC}^{\text {iPr }}-\mathrm{CO}_{2} ; \mathrm{NHC2}\right), \quad$ 1,3-bis(dodecyl)imidazolium carboxylate $\left(\mathrm{NHC}^{\text {Doc }}-\mathrm{CO}_{2} ; \mathrm{NHC} 4\right)$ and 1,3-bis(mesityl)imidazolium carboxylate $\left(\mathrm{NHC}^{\text {Mes }}-\mathrm{CO}_{2}\right.$; $\mathrm{NHC7)}$, was successfully achieved by Taton et al.[123] With $0.03-1.5$ eq. of $\mathrm{NHC}^{-\mathrm{CO}_{2}}$ adduct, PLA's with molar masses $M_{n}$ of $5,000 \mathrm{~g} \cdot \mathrm{mol}^{-1}(\Theta=1.13)$ were obtained. No induction period was noted with such $\mathrm{NHC}-\mathrm{CO}_{2}$ adducts, suggesting that the $\mathrm{NHC}$ was generated at r.t. Hence, under such experimental conditions, these precursors did not exhibit a latent behavior.

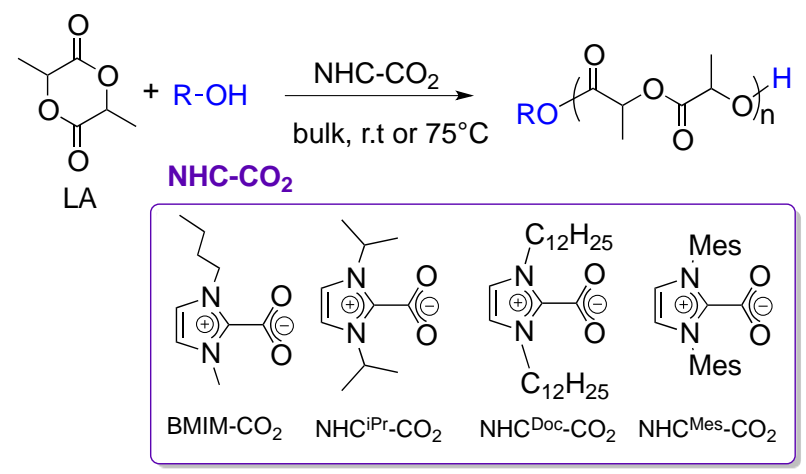

Scheme 34. ROP of LA using $\mathrm{NHC}-\mathrm{CO}_{2}$ pre-catalysts and $\mathrm{ROH}$ as initiators.[122, 123]

A poor efficiency of $\mathrm{NHC}^{\text {iPr }}-\mathrm{CO}_{2}, \mathrm{NHC}^{\text {Doc }}-\mathrm{CO}_{2}, \mathrm{NHC}^{\text {Mes }}-\mathrm{CO}_{2}$ for the $\mathrm{ROP}$ of $\varepsilon-\mathrm{CL}$ was noted at r.t., presumably due to the limited solubility of these pre-catalysts in the monomer.[123] In contrast, full conversion of $\varepsilon-\mathrm{CL}$ was observed at $75^{\circ} \mathrm{C}$ using $\mathrm{BMIM}-\mathrm{CO}_{2}$, leading to linear and 
star-like PCL oligomers $\left(\mathrm{M}_{\mathrm{n}}=2,300-11,700 \mathrm{~g} \cdot \mathrm{mol}^{-1} ; \quad \Xi=1.2-2.2\right) \cdot[122]$ Other $\mathrm{NHC}-\mathrm{CO}_{2}$ adducts, including imidazolium- (NHCs 2-3; 5-8), imidazolinium- (NHC25), tetrahydropyrimidinium-(NHCs 34-38) and tetrahydrodiazepinium-carboxylates (NHCs 39-40), bearing alkyl- and aryl-substituents (see Figure 2 for related structures), were also screened by Buchmeiser et al. for the ROP of $\varepsilon-\mathrm{CL}$, in presence of $\mathrm{BnOH}$ as initiator at $70{ }^{\circ} \mathrm{C}$.[124]

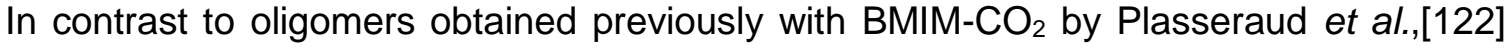
PCL's of $M_{n}=5,000-17,000$ g.mol-1 ( $\left.\theta=1.20-1.70\right)$ were achieved with this series of NHC$\mathrm{CO}_{2}$ adducts.[124] Less hindered imidazolium- (NHCs 2-3) and imidazolinium (NHC25) carboxylates were the most active for the ROP of $\varepsilon$-CL upon decarboxylation. Importantly, these protected NHCs were completely inactive at r.t., while a fast and quantitative consumption of the monomer was noted at $70^{\circ} \mathrm{C}$, evidencing the thermally latent behavior of these precatalysts. A nucleophilic AMM (Scheme 2) was postulated, but the occurrence of an ACEM (Scheme 4a) could not totally be ruled out. The latter mechanism was likely even more favored with NHCs derived from tetrahydropyrimidinium carboxylates, due to the higher basicity of the related free $\mathrm{NHCs}$ compared to that of imidazol-2-ylidenes ( $\mathrm{pKa}^{\mathrm{DMSO}}=27.8-28.2$ for tetrahydropyrimidin-2-ylidenes $v s$. $\mathrm{pKa}^{\mathrm{DMSO}}=20-25$ for imidazol-2-ylidenes)[94]. It was noted that 7-membered ring tetrahydrodiazepinium-carboxylates were ineffective pre-catalysts in these conditions.

The ROP of PO catalyzed by imidazolium $\left(\mathrm{NHC}^{\mathrm{Me}}-\mathrm{CO}_{2}, \mathrm{NHC}^{\mathrm{iPr}}-\mathrm{CO}_{2}, \mathrm{NHC}^{\mathrm{tBu}}-\mathrm{CO}_{2} \mathrm{NHC}^{\mathrm{Mes}}-\right.$ $\mathrm{CO}_{2}$ ), imidazolinium (NHC25) or benzimidazolium (NHC27) carboxylates was also reported.[125] Diethylene glycol (DEG) was employed as initiator, in bulk at $120^{\circ} \mathrm{C}$. In most cases, diol-functionalized PPO oligomers were obtained after $4 \mathrm{~h}$ (40-74\% of conversion), with $M_{n}=300-1,200 \mathrm{~g} \cdot \mathrm{mol}^{-1}$ and $\Theta=1.08-1.23$. Catalytic activity of $\mathrm{NHC}^{\mathrm{iPr}}-\mathrm{CO}_{2}$ was close to that observed in a previous work by Taton et al., with the corresponding free NHC.[126] Imidazolium pre-catalysts were by far the most active (the least-bulky substituents affording the best activity, $\mathrm{NHC}^{\mathrm{Me}}-\mathrm{CO}_{2}>\mathrm{NHC}^{\text {iPr }}-\mathrm{CO}_{2}>\mathrm{NHC}^{\mathrm{tBu}}-\mathrm{CO}_{2}>\mathrm{NHC}^{\text {Mes }}-\mathrm{CO}_{2}$ ), compared to the benzimidazol-type (NHC27), which proved ineffective. The same system was also efficient for the sequential ROP of $\mathrm{PO}$ and $\varepsilon-\mathrm{CL}$ and $\mathrm{L}-\mathrm{LA}$, leading to poly(ether)-b-poly(ester) copolymers of low $\mathrm{M}_{\mathrm{n}}\left(750-3,700 \mathrm{~g} \cdot \mathrm{mol}^{-1}\right.$ and $\left.\Xi=1.38-1.77\right)$.

Based on DFT calculations and in situ IR spectroscopy, it was proposed that decarboxylation was a slow process, which allowed a continuous delivery of the free carbene catalyst, thus providing a better control over the ROP. ROP of PO would occur via the AMM by the NHC, forming a zwitterionic imidazolium alkoxide 21 (Scheme 35). The latter species would be subsequently displaced by the $\mathrm{ROH}$, generating an $\alpha$-alkoxide monoadduct $\mathbf{2 3}$, whereas an imidazolium would be released, the polymerization proceeding through an anionic mechanism (route $A_{1}$ ). Such a mechanism contrasted with that proposed by Taton et al. where NHC 2 could serve either as catalyst (route $A_{2}$ ) or as direct initiator (route B).[126] Further investigations 
would be definitely needed, however, to clarify the precise mechanism, which could eventually depend on the structure of the NHC catalyst.

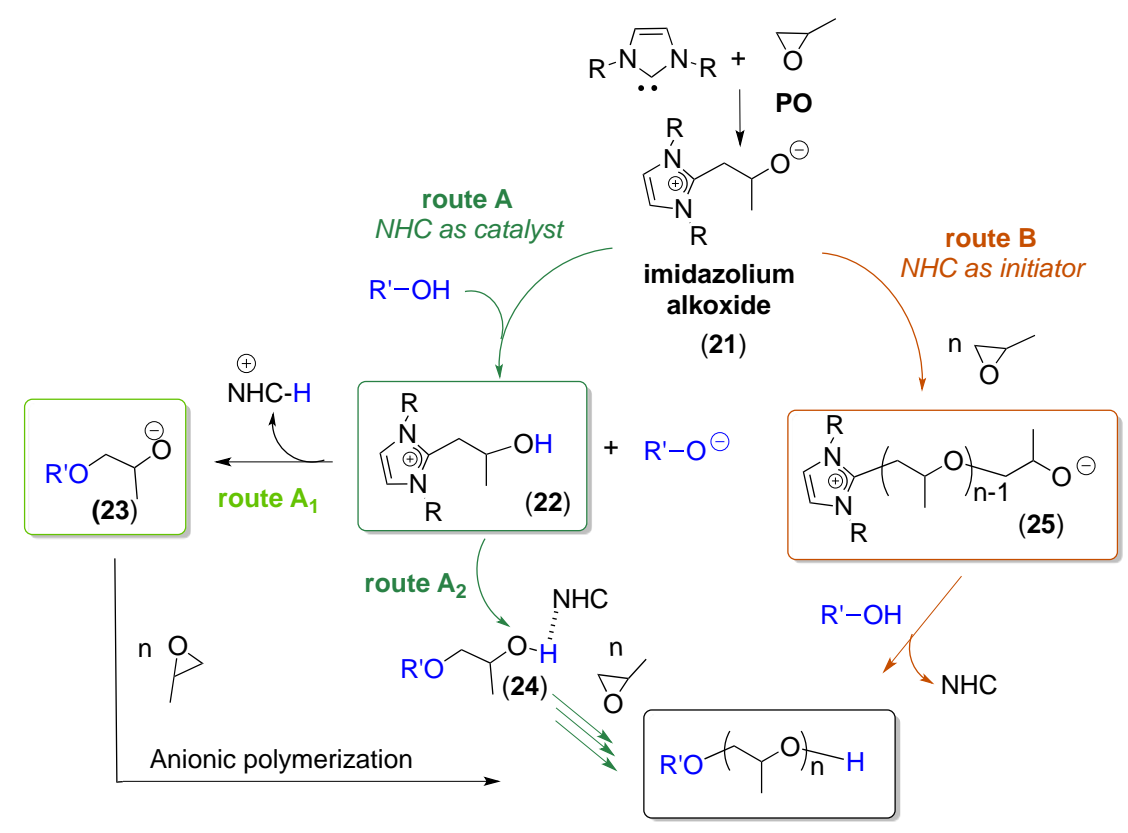

Scheme 35. Possible mechanisms for the NHC-catalyzed ROP of PO in presence of an alcohol initiator.[111, 126]

$\mathrm{NHC}-\mathrm{CO}_{2}$ adducts were also used to trigger the ROP of cyclic carbonates. For instance, $\mathrm{NHC}^{\text {iPr }}-\mathrm{CO}_{2}(0.1 \mathrm{~mol} \%$ relative to the initiator) was found particularly active for the ROP of TMC in THF at r.t., using $\mathrm{BnOH}$ as initiator.[123] The polymerization reached $91 \%$ of $\mathrm{TMC}$ conversion after $1 \mathrm{~h}$, leading to a PTMC of $\mathrm{M}_{n}=3,500 \mathrm{~g} \cdot \mathrm{mol}^{-1}(\Theta=1.07)$, confirming that the free NHC was generated under such conditions. TMC was also polymerized in bulk and in THF or toluene solution at 60,80 and $100{ }^{\circ} \mathrm{C}$, in the presence of $\mathrm{NHC}^{\text {Mes }}-\mathrm{CO}_{2}$ as pre-catalyst and $\mathrm{BnOH}$ as initiator.[127] While at $60^{\circ} \mathrm{C}$ and $80^{\circ} \mathrm{C}$, solvent-free polymerizations were quite similar, giving a PTMC of $M_{n}=11,000 \mathrm{~g} \cdot \mathrm{mol}^{-1}(\Theta=1.4)$, slightly higher molar masses $\left(M_{n}=13,400 \mathrm{~g} \cdot \mathrm{mol}^{-1}\right)$ and a broader dispersity $(\Theta=1.7)$ were obtained at $100^{\circ} \mathrm{C}$. Polymerizations in solution at $60^{\circ} \mathrm{C}$ were less controlled, yielding PTMC's of lower molar masses $\left(M_{n}=2,000-3,000 \mathrm{~g} \cdot \mathrm{mol}^{-1}\right)$ with higher dispersities $(\theta=1.3-3.0)$. These observations evidenced the temperature-dependence of this NHC-catalyzed ROP of TMC, with decarboxylation of the monomer occurring at high temperatures.

Taton et al. reported the use of $\mathrm{NHC}^{\mathrm{iPr}}-\mathrm{CO}_{2}$ as an efficient pre-catalyst also for the GTP of MMA, using MTS as initiator at r.t., both in bulk and THF.[123] With 3 mol\% of $\mathrm{NHC}^{\mathrm{iPr}}-\mathrm{CO}_{2}$ (relative to MTS), PMMA's of molar masses $M_{n}$ of $6,300 \mathrm{~g} \cdot \mathrm{mol}^{-1}$ and $\Theta=1.12-1.37$, with a good control over the polymerization, were obtained.

The same group described the ROP of D,L-LA using imidazolium hydrogen carbonates, denoted as $[\mathrm{NHC}(\mathrm{H})]\left[\mathrm{HCO}_{3}\right]$, as pre-catalysts $(10 \mathrm{~mol} \%$ relative to the initiator) and $\mathrm{BnOH}$ as initiator.[128] The saturated $\left[\mathrm{NHC}^{\mathrm{Mes}}(\mathrm{H})\right]\left[\mathrm{HCO}_{3}\right]\left(\mathrm{NHC}^{25}\right.$ in Figure 2) precursor was found to be 
the most efficient precursor, high monomer conversions of lactide being achieved in THF at r.t., resulting in PLA's of molar masses $\left(\mathrm{M}_{\mathrm{n}}=4,000-7,800\right.$ g. $\left.\mathrm{mol}^{-1} ; \Theta<1.07\right)$. However, catalytic efficiencies of $[\mathrm{NHC}(\mathrm{H})]\left[\mathrm{HCO}_{3}\right]$ were approximately three times lower for the ROP of D,L-lactide than with $\mathrm{NHC}-\mathrm{CO}_{2}$ counterparts, and obviously lower than free (bare) NHCs. Overall, the activity of these catalysts could be ranked as follows: $\left([\mathrm{NHC}(\mathrm{H})]\left[\mathrm{HCO}_{3}\right]<\mathrm{NHC}-\mathrm{CO}_{2}\right.$ adducts < free NHCs). In comparison with $\mathrm{NHC}-\mathrm{CO}_{2}$ adducts, $[\mathrm{NHC}(\mathrm{H})]\left[\mathrm{HCO}_{3}\right]$ were not effective for the solvent-free GTP of MMA using MTS as initiator at r.t., owing to a loss of control over the polymerization, because of the poor solubility of the salt precursor in the monomer substrate.[123]

A NHC exhibiting a phtotoswitchable activity in response to light irradiation, was reported by Bielawski et al. Such a latent photocatalytic system was employed for the ROP of $\varepsilon$-CL and $\delta$-VL initiated by $\mathrm{BnOH}$ in THF at r.t (Scheme 36).[129] Polymerizations proceeded efficiently in natural light, producing PCL's and PVL's of controlled molar masses $\left(M_{n}\right.$ up to 15,900 g.mol ${ }^{-1}$; $\Xi=1.15$ ), after $30 \mathrm{~min}$ of reaction. Upon UV irradiation, the polymerization rate was attenuated, due to the formation of the photocyclized NHC-alcohol adduct (NHC-C, Scheme 36), that was catalytically inactive, due to the decreased electron density at the carbenoid center. Subsequent visible light exposure reversed the photocyclization, regenerating the active imidazolium species (NHC-O).

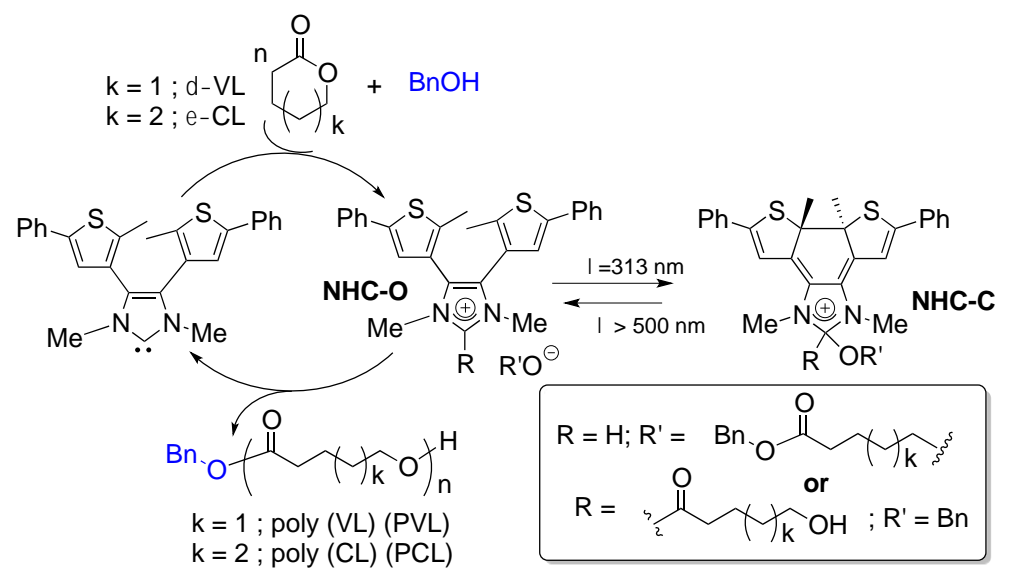

Scheme 36. Catalytic cycle for the photoswichtable NHC-catalyzed ROP of $\varepsilon-\mathrm{CL}$ and $\delta$ - $\mathrm{VL}$ in presence of $\mathrm{BnOH}$ initiator.[129]

In conclusion, NHCs have been successfully used as organic catalysts for chain-growth polymerization, including ROP of cyclic esters, carboxyanhydrides, as well as for the synthesis of polyurethanes via step-growth polymerizations. Protected NHCs provide a genuine source of carbenes for metal-free polymerizations. However, their catalytic efficiencies often remain lower than the corresponding free $\mathrm{NHCs}$, probably due to a slow or incomplete generation of active species under polymerization conditions. Further developments towards air-stable protected NHCs, which could be efficiently activated by an external stimulus, could further expand the scope of NHC-catalyzed polymerization reactions. 


\section{Polymerization catalyzed by nitrogen-containing Brønsted and Lewis bases: alkyl amines, amidines and guanidines}

\subsection{Alkyl amines and pyridine derivatives}

In comparison with NHCs, pyridine derivatives, such as dimethyaminopyridine (DMAP) and alkyl(aryl) amines are relatively moderate Brønsted bases, but they can behave as powerful nucleophiles.[130] These nitrogen-containing organocatalysts have been used for the ROP of various heterocycles, including lactide, lactones, carbonates and $O$-carboxyanhydrides. An overview of recent polymerization reactions catalyzed by amines as well as related pKa values are illustrated in Figure 14.

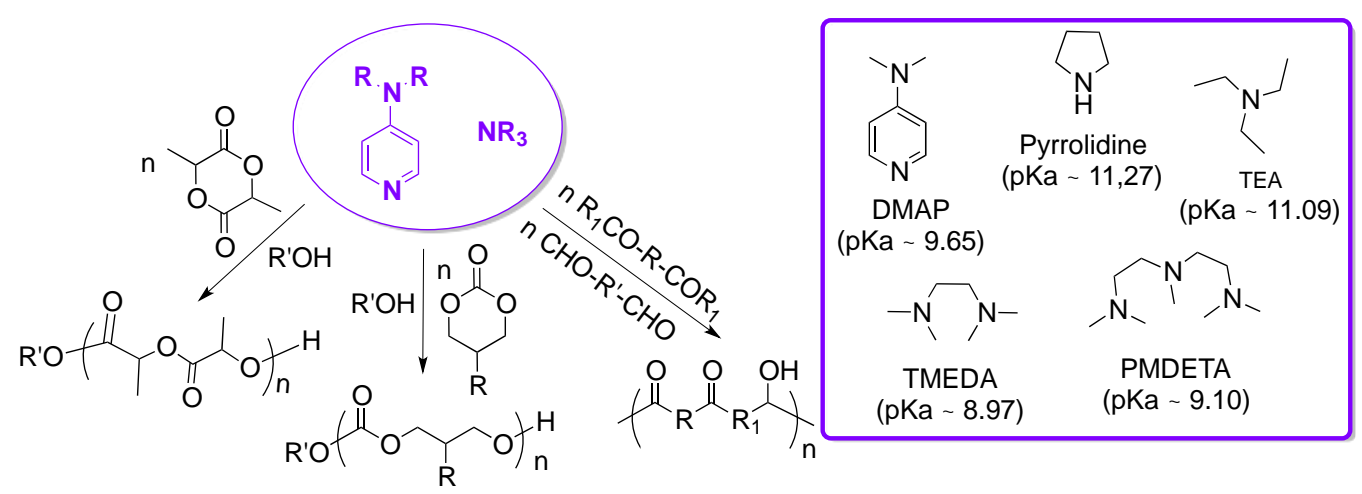

Figure 14. Scope of amine-catalyzed polymerization reactions and representative catalysts ( $\mathrm{pKa}$ values are given in $\mathrm{H}_{2} \mathrm{O}$ ).

DMAP (pKa 9.65 in $\mathrm{H}_{2} \mathrm{O}$ ) was used as catalyst by Zinck et al. for the ROP of D,L-lactide using carbohydrate-based initiators, such as glucose and cyclodextrin derivatives $(\mathrm{DMAP} / \mathrm{ROH})=2$.[131] According to the solubility of the initial carbohydrate, polymerizations were conducted in solution (dichloromethane or chloroform) at $35^{\circ} \mathrm{C}$ or in bulk at $120^{\circ} \mathrm{C}$. Carbohydrate-functionalized polylactides of number-average DP ranging from 2 to 210 $(\Theta=1.07-1.48)$, considering the growth of one polymeric chain per initiating hydroxyl group, were obtained with a high functionalization efficiency.

In the same manner, DMAP served as catalyst for the copolymerization of L-LA and TMC in toluene at $130^{\circ} \mathrm{C}$, using $\mathrm{BnOH}$ as initiator.[75] The ROP of L-LA, using a pre-synthesized $\mathrm{BnO}-\mathrm{PTMC}-\mathrm{OH}$ prepolymer or through direct sequential copolymerization of the two monomers, afforded PTMC-b-PLLA diblock copolymers of molar masses, $M_{n}$, of $26,200 \mathrm{~g} \cdot \mathrm{mol}^{-1}$ ( $\Theta=1.32$ ). DMAP was thought to operate via an ACEM (Scheme 4a). Under the same conditions, the phosphazene BEMP was more effective than DMAP, presumably due to its higher basicity. 
Aliphatic tertiary amines, including triethylamine (TEA; pKa 11.09 in $\mathrm{H}_{2} \mathrm{O}$ ), N,N,N,Ntetramethylethylenediamine (TMEDA; pKa 8.97 in $\mathrm{H}_{2} \mathrm{O}$ ) and $N, N,-N, N, N$-pentamethyl diethylenetriamine (PMDETA; $\mathrm{pKa} \sim 9.10$ in $\mathrm{H}_{2} \mathrm{O}$ ), were used to catalyze the $\mathrm{BnOH}$ initiatedROP of TMC in THF at $55^{\circ} \mathrm{C}$ (Scheme 37).[132] Under those conditions, PMDETA and TMEDA were the most efficient catalysts, yielding PTMC's of $M_{n}=1,600-10,400$ g. mol ${ }^{-1}$ and $\unrhd=1.17$ 1.55, at full TMC conversion. The catalytic activity correlated not only to the basicity of tertiary amines and to their ability to activate the initiating alcohol by $\mathrm{H}$-bonding, but also with the number of nitrogen atoms in the catalyst (see insert in Scheme 37).[133] A particular spatial orientation of nitrogen lone pairs was proposed to explain the catalytic activity of the amines. According to NMR characterizations, this amine-catalyzed ROP of TMC presumably proceeded through the ACEM (Scheme 4a). Using a $\mathrm{PEO}_{99}-\mathrm{PPO}_{65}-\mathrm{PEO}_{99}-\mathrm{OH}$ macroinitiator, poly(ether-carbonate) copolymers with a well-defined structure could be easily prepared by this amine-catalytic pathway.

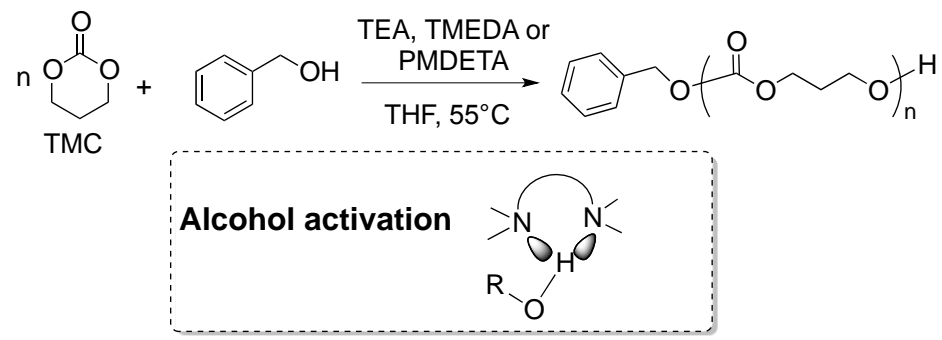

Scheme 37. ROP of TMC catalyzed by tertiary amines (TMEDA or PMDETA) and initiated by $\mathrm{BnOH} .[132]$

\subsection{Amidines and guanidines}

Compared to trialkyl or aryl amines, amidines and guanidines are stronger organic bases (pKa 23-26 in MeCN)[134], due to the stability of their corresponding conjugated acid (the positive charge is delocalized over two or three nitrogen atoms).[66] Amidines and guanidines are however slightly less basic (by approximately 2-3 pKa units) than phosphazenes such as 2tert-butylimino-2-diethylamino-1,3-dimethylperhydro-1,3,2-diazaphosphorine (BEMP in Figure 8; $\mathrm{pKa} \sim 27.6$ in MeCN). As phosphazenes, amidines and guanidines have been mainly used as deprotonating agents of acidic $\mathrm{O}-\mathrm{H}, \mathrm{S}-\mathrm{H}$ or $\mathrm{C}-\mathrm{H}$-containing initiators (see section 3.1), but a particular guanidine, namely, 1,5,7-triazabicyclo[4.4.0]dec-5-ene (TBD) (pKa $\left.{ }^{\mathrm{MeCN}}=26.0\right)[134]$ could also serve as dual activator in some molecular or macromolecular reactions. $[7,8,14]$ Representative examples of guanidines and amidines employed in metal-free polymerizations are shown in Figure 15. 


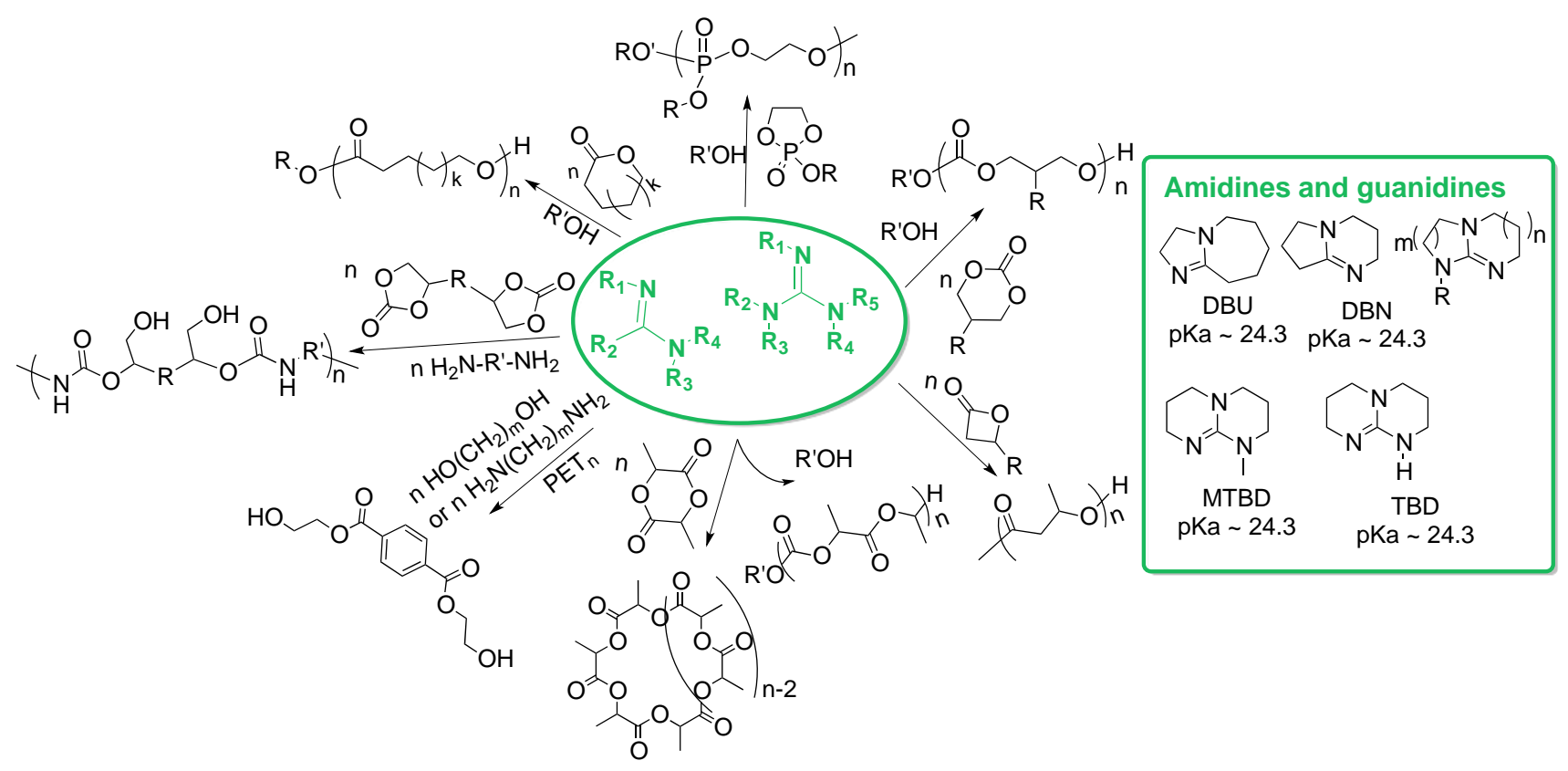

Figure 15. Scope of polymerization reactions catalyzed by amidines and guanidines (pKa values are given in MeCN [134]).

\subsubsection{Amidines}

The amidine 1,8-diazabicyclo[5.4.0]undec-7-ene (DBU) (pKa = 24.3 in MeCN)[134] was found to be an excellent Brønsted basic catalyst for the ROP of lactide in presence of alcohol initiators.[7, 8, 14] In contrast, the polymerization of lactones required the use of a thiourea cocatalyst to minimize transesterifications.[135]

Based on previous results obtained for DBU-catalyzed ROP of LA,[7] Coulembier et al. explored the bulk polymerization of the eutectic L-LA/TMC (50/50) melt, using $\mathrm{BnOH}$ as initiator, at $23^{\circ} \mathrm{C}$ (Scheme 38).[136] Pure homoPLLA's of molar masses $M_{n}=15,500 \mathrm{~g} \cdot \mathrm{mol}^{-1}(\Theta=1.33)$ were obtained from this particular mixture of monomers. This was attributed to the limiting diffusion of TMC during the very fast ring-opening process of LA (conversion of $95 \%$ in $30 \mathrm{~s}$ ), resulting from PLLA crystallization out of the eutectic melt. Nevertheless, ROP of TMC was observed when the medium was quickly solubilized in dichloromethane after PLLA formation, affording poly(LLA-g-TMC) gradient copolymers of $M_{n}=22,000 \mathrm{~g} \cdot \mathrm{mol}^{-1}$ and $\oslash=1.64$ after $16 \mathrm{~h}$ (63\% of TMC conversion)

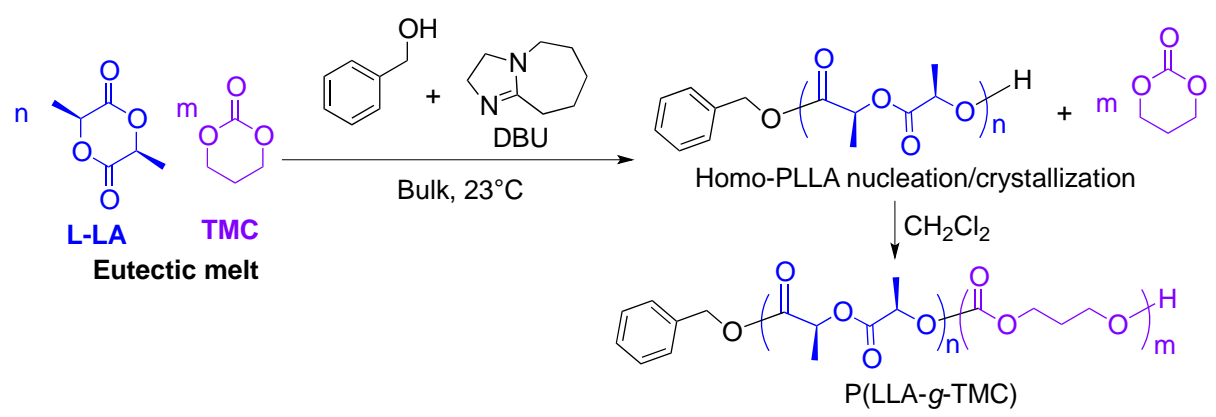


Scheme 38. DBU-catalyzed ROP of eutectic melt of L-LA and TMC monomers initiated by $\mathrm{BnOH} .[136]$

ROP of a particular cyclic carbonate, namely, spiro[fluorene-9,5'-[1,3]-dioxan]-2'-one (F-TMC) was also reported by Hedrick et al., using $\mathrm{BnOH}$ as initiator and $\mathrm{DBU}$ as catalyst (1eq) (Scheme 39).[137] Polymerizations proceeded in dichloromethane at r.t, leading to poly ( $\mathrm{F}$ TMC)'s of predictable molar masses $\left(\mathrm{M}_{\mathrm{n}}\right.$ of to $\left.4,500 \mathrm{~g} \cdot \mathrm{mol}^{-1} ; \ominus=1.3\right)$. When employing MeOPEO-OH of different molar masses $\left(M_{n}=1,600-10,000\right.$ g.mol $\left.{ }^{-1} ; €<1.2\right)$ as macroinitiators, the polymerization was almost completed in $15 \mathrm{~min}$ (monomer conversion > 95\%), yielding welldefined amphiphilic diblock copolymers of $M_{n}=5,000 \mathrm{~g} \cdot \mathrm{mol}^{-1}$ for the poly(F-TMC) block, as determined by NMR. Depending on the molar mass of the PEO blocks, spheres or elongated rod-like micelles or lamellar phases such as tapes could be generated by self-assembly of these copolymers in aqueous solution.

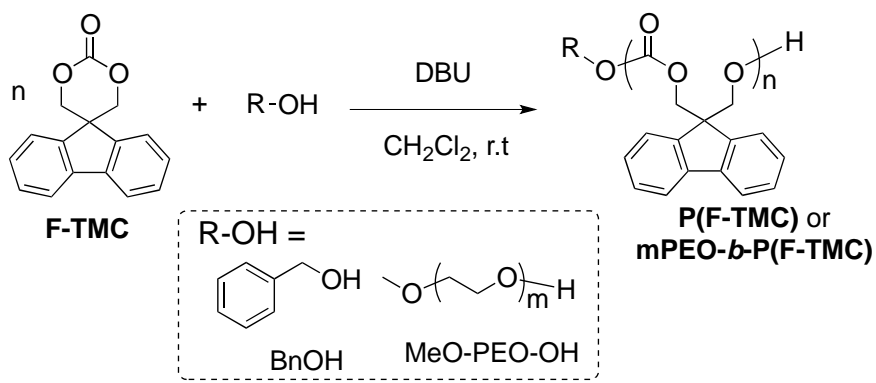

Scheme 39. DBU-catalyzed ROP of fluorene-functionalized aliphatic cyclic carbonate monomer.[137]

Besides polyesters and polycarbonates, polyphosphoesters are attractive materials for bioapplications because they also exhibit biocompatible and biodegradable properties.[138] In this context, Iwasaki et al. reported, in 2010, the first solvent-free ROP of cyclic phospholanes such as 2-isopropoxy-2-oxo-1,3,2-dioxaphospholane (iPP) and 2-methacryloyloxyethylphosphoryl choline (PiPP) using DBU as catalyst, in presence of alcohols as initiators.[139] Polyphosesters of $\mathrm{M}_{n}=2,400-7,700 \mathrm{~g} \cdot \mathrm{mol}^{-1} \quad(\Theta=1.03-1.09)$ were produced and the polymerization was thought to occur by a basic ACEM, on the basis of NMR analysis.

In the same way, Wooley et al. described the DBU-catalyzed ROP of butynyl phospholane (BYP) in dichloromethane at r.t., using $\mathrm{BnOH}$ as initiator (Scheme 40a).[140] Using 1.5 eq. of DBU relative to $\mathrm{BnOH}$, poly(BYP)'s of molar masses $M_{n}$ of 20,000 g.mol-1 $(~(D=1.14-1.19)$ containing a pendent alkyne function were obtained after only 6 min (conversion of $95 \%$ ). Postfunctionalization of the obtained polyphosphoesters was accomplished via azide-alkyne Huisgen cycloaddition and thiol-yne reactions.

The ROP of 2-isobutoxy-2-oxo-1,3,2 dioxaphospholane (iBP) was initiated in toluene at $0^{\circ} \mathrm{C}$ using 1eq. of $\mathrm{BnOH}$ as initiator in presence of 5 eq. of DBU (Scheme 40a).[141] Although $M_{n}$ of the obtained poly(iBP)'s ( $\left.M_{n}=6,900-15,800 \mathrm{~g} \cdot \mathrm{mol}^{-1} ; \ominus=1.19-1.32\right)$ evolved with the monomer conversion, this polymerization was very slow $(24 \mathrm{~h}$ to achieve $80 \%$ for $\mathrm{DP}=50$ ) and a multimodal molar mass distribution was observed, especially for high monomer conversion 
and long polymerization time. This was explained by the occurrence of side reactions, such as intra- and/or intermolecular transesterifications. Sequential copolymerization of BYP and L-LA (BYP was first polymerized) gave a poly(butynyl phospholane)- $b$-poly(lactide) (PBYP- $b$-PLLA) diblock copolymer of $M_{n}=21,900 \mathrm{~g} \cdot \mathrm{mol}^{-1}$ and $\Xi=1.17$.[142] After suitable modifications, the amphiphilic copolymer was manipulated to self-assemble into spherical nanoparticles, with distinct negative and positive surface charges, and uniform size distributions.

a)<smiles>[2H]P[PH2](=O)OCCO</smiles>
Phospholanes $\mathrm{R}=\mathrm{CH}_{3} ; \mathbf{M P}$ $\mathrm{R}=\mathrm{CH}_{2} \mathrm{CH}\left(\mathrm{C}_{2} \mathrm{H}_{5}\right)_{2} ; \mathrm{EBP}$ $\mathrm{R}=\left(\mathrm{CH}_{2}\right)_{2}-\mathrm{CCH} ; \mathrm{BYP}$ $\mathrm{R}=\mathrm{CH}_{2} \mathrm{CH}\left(\mathrm{CH}_{3}\right)_{2} ; \mathrm{iBP}$ b)

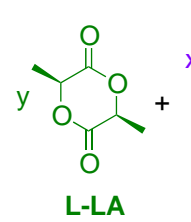

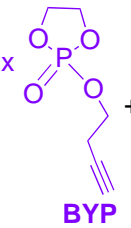<smiles>OCc1ccccc1</smiles>

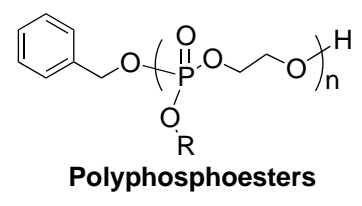
or toluene, $0^{\circ} \mathrm{C}$

Polyphosphoesters

BYP

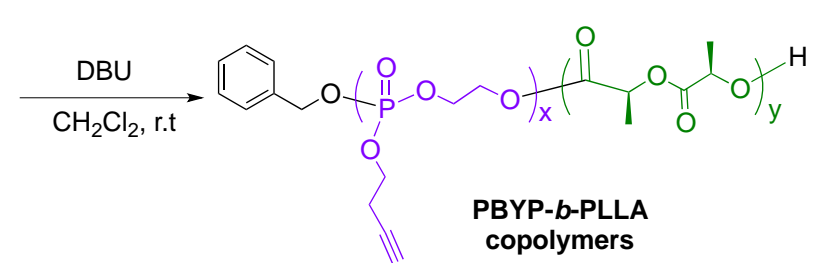

Scheme 40. DBU (or TBD)-catalyzed ROP of phospholanes and copolymerization with L-LA using alcohol initiators.[140-142] scheme modified

\subsubsection{Guanidines}

The most studied bicyclic guanidine, 1,5,7-triazabicyclo[4.4.0]dec-5-ene (TBD), $\mathrm{pKa}=26.0$ in MeCN,[134] has been established as a powerful catalyst for the ROP of cyclic esters and cyclic carbonates.

In presence of alcohol initiators, TBD was reported to specifically operate as a bifunctional activator (Scheme 41). Both the alcohol and the carbonyl groups of the monomer are activated by $\mathrm{H}$-bonding in a cooperative fashion, creating concomitantly an incipient guanidinium ion (see also section 6 for other examples of organic catalyst providing a dual activation).[7, 14] TBD is discussed here for the purpose of comparison with other guanidines or amidines.

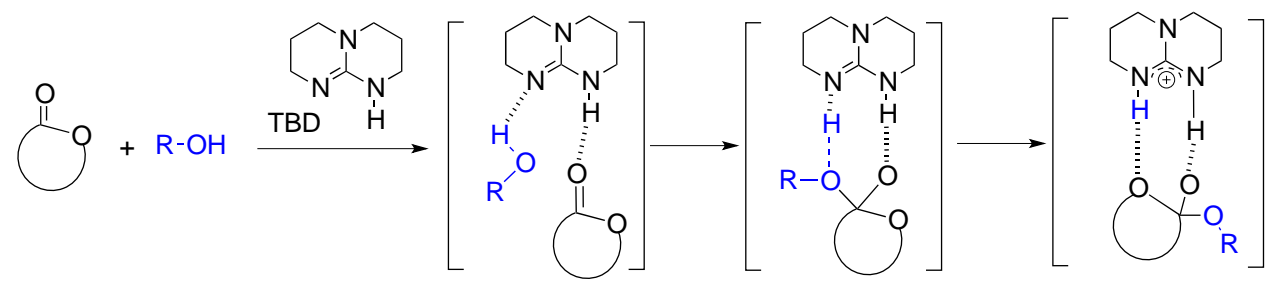

Scheme 41. Activation mode of TBD catalyst during the ROP of cyclic esters.[14]

Hillmyer et al. reported the solvent-free ROP of the renewable $\delta$-decalactone ( $\delta$-DL), using 1,4-phenylenedimethanol (PDM) diol as initiator and TBD as catalyst (0.3 eq).[143] At $100{ }^{\circ} \mathrm{C}$, full monomer conversion was reached, leading to high molar masses PDL's $\left(M_{n}\right.$ of 83,000 g.mol' 
$1 ; \oplus<1.25)$. Sequential addition of L-LA, and $\delta$-DL produced PLLA- $b$-PDL- $b$-PLLA triblock copolymers $\left(M_{n}=100,000 \mathrm{~g} \cdot \mathrm{mol}^{-1} ; \quad D=1.4\right)$, that exhibited two distinct glass transition temperatures, at -51 and $54{ }^{\circ} \mathrm{C}$ for PDL and PLLA domains, respectively, consistent with a microphase segregation.

Similarly, starting from either 1-pyrenemethanol or PEO-OH as macroinitiator, the TBDcatalyzed ROP of LA and $\varepsilon-C L$ was investigated in bulk at $90^{\circ} \mathrm{C}$.[144] This allowed synthesizing PCL's and PLA's, as well as PEO- $b$-PCL(or PLA) diblock and amphiphilic PEO- $b-\mathrm{P}$ (CL-co-LA) triblock copolymers of molar masses $M_{n}$ in the range of $3,500-23,700 \mathrm{~g}^{-\mathrm{mol}^{-1}}(\Theta=1.4-2.0)$. These amphiphilic copolymers were shown to self-organize into micelles of hydrodynamic radius $\mathrm{R}_{\mathrm{h}}=27-35 \mathrm{~nm}$.

The ROP of $\delta$-VL catalyzed by TBD (0.17 eq), using $\mathrm{BnOH}$ as initiator (1 eq.) was reported in toluene at r.t.[145] The corresponding PVL of $M_{n}=3,000 \mathrm{~g} \cdot \mathrm{mol}^{-1}(\Xi=1.06)$ was obtained within 15 min of reaction for an initial $D P=30$. Addition of a crosslinking agent, such as and [4,4'-bioxepane]-7,7'-dione (BOD) to this PVL macroinitiator, led to a polyester nanogel star of $M_{n}=333,000 \mathrm{~g} \cdot \mathrm{mol}^{-1}(\Theta=1.21)$. Alternatively, and more practically, such branched polyesters could also be obtained by mixing all reagents at the early stage of the reaction.

a)<smiles>O=C1CCCCC1</smiles>
$-V L$

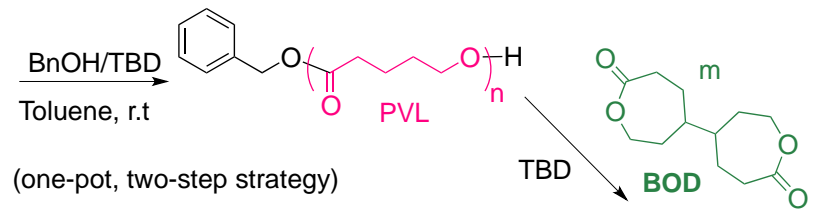

b)
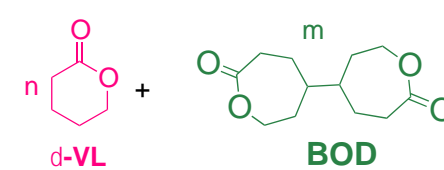

BOD

$\underset{\text { Toluene, r.t }}{\stackrel{\mathrm{BnOH} / \mathrm{TBD}}{\longrightarrow}}$

(one-pot, one-step strategy)

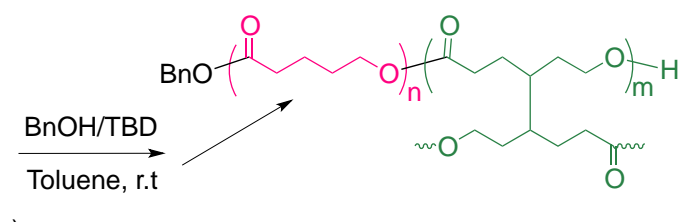

Scheme 42. Synthesis of a polyester-based nanogel star polymer by TBD-catalyzed ROP of $\delta$-VL and BOD initiated by $\mathrm{BnOH}$ : a) one-pot/two-step strategy and b) one-pot/one step strategy.[145]

The TBD-catalyzed ROP of functionalized lactones, namely, 3-benzyl mercaptovalerolactone (BMVL), 2-(2-methoxyethoxy) ethanethiolvalerolactone ( $\mathrm{GG}_{2} \mathrm{MVL}$ ) and 1mercapto-3,6,9,12-tetraoxotridecanevalerolactone (EG $4 \mathrm{MVL})$ (Scheme 43a), was also described.[146] 1,4-Pyrenebutanol (PyBuOH) was used as initiator (1-5 mol\% of TBD relative to the alcohol) and reactions were carried out in bulk or in toluene at r.t.[146] Well-defined functionalized polyesters $\left(M_{n}=500-8,900 \mathrm{~g} \cdot \mathrm{mol}^{-1} ; \quad Ð=1.32-1.45\right)$, featuring benzyl mercaptans or oligoethylene glycol pendant groups, could thus be obtained. In addition, aliphatic functionalized polyesters $\left(M_{n}=5,800-6,900 \mathrm{~g} \cdot \mathrm{mol}^{-1} ; \quad \nexists=1.21-1.47\right)$ were successfully prepared by the copolymerization of 3-mercaptovalerolactones with $\varepsilon$-CL (Scheme 43b). 
a)
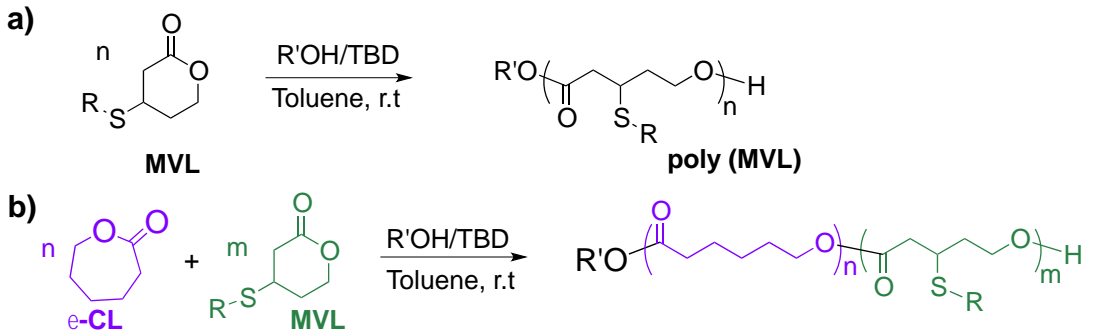

Scheme 43. TBD-catalyzed: a) ROP of substituted valerolactones (MVL); b) random copolymerization of MVL and $\varepsilon-C L .[146]$

In the same manner, various functionalized lactones, including $\alpha$-allyl- $\delta$-valerolactone (AVL), $\alpha$-propargyl- $\delta$-valerolactone (PgVL), TMS-protected PgVL (TMS-PgVL), and $\alpha$ propargyl- $\delta$-caprolactone (PgCL), could be polymerized at $r$.t in toluene, using $\mathrm{BnOH}$ and TBD (2 mol\% of relative to the monomer) as initiator and catalyst, respectively.[147] Functionalized aliphatic homopolymers, as well as copolymers of molar masses $M_{n}=8,800-22,000 \mathrm{~g} \cdot \mathrm{mol}^{-1}$ and $\Theta=1.01-1.23$, incorporating pendant alkene and alkyne groups were prepared. Postpolymerization modification of these polyesters by "click-type" chemistries, allowed hydrophilic solubilizing groups and fluorescent moieties to be incorporated for delivery and controlled release applications.

As an example of base-mediated polymerization of macrolactones, ROP of $\omega$ pentadecalactone ( $\omega$-PDL) and its copolymerization with $\varepsilon$-CL was investigated by Duchateau el al.[148] The combination of $\mathrm{TBD} / \mathrm{BnOH}(1 / 1)$ proved catalytically active, in bulk or toluene at $100{ }^{\circ} \mathrm{C}$, producing PCL, PDL homopolymers $\left(\mathrm{M}_{\mathrm{n}}=2,500-27,000 \mathrm{~g} \cdot \mathrm{mol}^{-1} ; \bigoplus=1.3-2.1\right)$, as well as random poly(PDL-co-CL) copolymers $\left(\mathrm{M}_{n}=9,500\right.$ and $\left.11,500 \mathrm{~g} \cdot \mathrm{mol}^{-1} ; \Xi=1.5\right)$. At high monomer conversion, a broadening of the molar mass distribution was observed, suggesting the occurrence of transesterification reactions. Accordingly, a general ACEM was ruled out due to the inactivity of the methylated guanidine homologue, $\mathrm{N}$-methyl-1,5,7-triazabicyclododecene (MTBD) under such conditions. Although experimental evidences were lacking, a dual activation of monomer and growing polymer chain by TBD was the most likely mechanism for such macrolactone polymerization.

The TBD-catalyzed ROP of ethylene brassylate (EB) was also investigated by Mecerreyes et al., leading to polyester of $M_{n}=7,000-13,900 \mathrm{~g} \cdot \mathrm{mol}^{-1}(\Theta=1.6-1.9) \cdot[38$ ] Compared to the acid catalysis by sulfonic acids, the $\mathrm{TBD} / \mathrm{BnOH}$ combination proved the fastest system, and side reactions were limited. DFT calculations revealed that both TBD and PTSA acted as a bicomponent dual activating system via $\mathrm{H}$-bonding, but the polymerization was kinetically more favored when TBD was used.

In contrast to the numerous examples of ROP of lactones, only a few reports has described the ROP of lactide catalyzed by guanidines. For instance, the ROP of a PEO-based 
macromonomer featuring a lactide polymerizable group (PEO-LLA) has been reported, using $\mathrm{BnOH} / \mathrm{TBD}$ (1/0.5), in dichloromethane at r.t (Scheme 44).[149] PLA-g-PEO copolymers were thus obtained with molar masses $M_{n}=11,000-14,000 \mathrm{~g} \cdot \mathrm{mol}^{-1}$ and $Ð=1.4-2.1$.

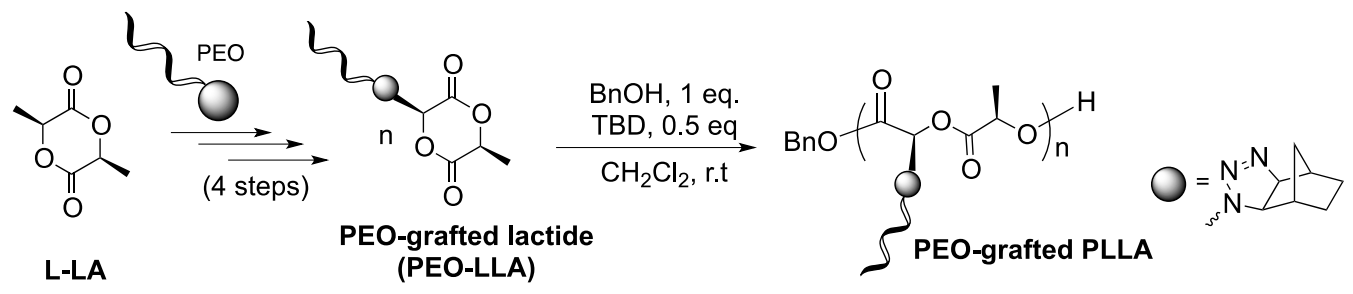

Scheme 44. Synthesis of PEO-grafted PLLA from L-lactide.[149]

Similarly to DBU, TBD could also catalyze the ROP of some phospholane monomers. In particular, ROP of 2-isobutoxy-2-oxo-1,3,2 dioxaphospholane (iBP) was carried out in toluene at $0{ }^{\circ} \mathrm{C}$, using $\mathrm{BnOH}$ as initiator (Scheme 40a).[141] In comparison with DBU, TBD provided faster reaction kinetics, complete conversion being obtained within only $2 \min$ (DP $=100,78 \%$ conversion after $11 \mathrm{~h}$ with DBU). However, TBD-derived polyphosphoesters poly(iBP)'s exhibited a bimodal molar masses distribution, presumably originating from chain coupling by intermolecular transesterification.

The sequential copolymerization of 2-ethylbutyl phospholane (EBP) and butynyl phospholane (BYP) (EBP being polymerized first) was performed in dichloromethane at $0{ }^{\circ} \mathrm{C}$ by Wooley et al. (Scheme 40a).[150] In this way, PEBP ${ }_{50}-b-$ PBYP $_{50}$ diblock copolymers $\left(\mathrm{M}_{\mathrm{n}}=7,100-17,200 \mathrm{~g} \cdot \mathrm{mol}^{-1} ; \bigoplus<1.17\right)$ could be prepared successfully. In contrast, DBU was reported to be only active towards the polymerization of BYP, potentially because of the bulkier ethylbutyl group in EBP. Post-functionalization of the above copolymers by thiol-yne reactions, with various thiols, resulted in nonionic, anionic, cationic and zwitterionic amphiphilic block copolymers that were shown to self-assemble into micelle-like nanostructures.

The TBD-catalyzed ROP of cyclic carbonates proved a practical synthetic strategy to polycarbonates with enhanced thermo-mechanical properties. For instance, Guillaume, Carpentier et al. reported the ROP of the L-LA/TMC binary system in toluene at $110^{\circ} \mathrm{C}$, using TBD in combination with $\mathrm{BnOH}(1 / 5)$.[151] Random P(LLA-TMC) copolymers of molar masses in the range $M_{n}=6,500-11,000 \mathrm{~g} \mathrm{~mol}^{-1}(\Theta=1.4-2.1)$ were thus obtained. Although this copolymerization proceeded without decarboxylation, extensive transesterification sidereactions were observed upon prolonged reaction time.

The same group achieved the ROP of racemic trans-cyclohexene carbonate (rac-CHC) in toluene at $60^{\circ} \mathrm{C}$, using TBD in combination with $\mathrm{BnOH}$ as co-initiator (Scheme 45a).[152] The reaction proved efficient (75-85\% conversion in 1-4 h), affording well-defined isotactic PCHC's with $M_{n}$ up to $18,100 \mathrm{~g} \cdot \mathrm{mol}^{-1}(\Theta=1.16-1.54)$. 
Glucose-derived polycarbonates $\left(M_{n}=4,100-13,000\right.$ g.mol $\left.{ }^{-1} ; \ominus=1.11-1.15\right)$ were also successfully produced in dichloromethane at r.t, by the TBD-catalyzed ROP of a glucose-based cyclic carbonate initiated by 4-methylbenzyl alcohol (Scheme 45b).[153] DSC analysis of these polycarbonates revealed glass transition temperatures significantly higher than those of PTMC $\left(106{ }^{\circ} \mathrm{C}\right.$ for glucose-polycarbonate vs. $-18^{\circ} \mathrm{C}$ for PTMC).

a)

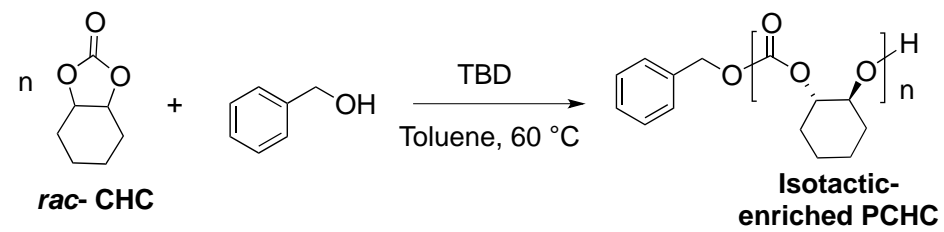

b)

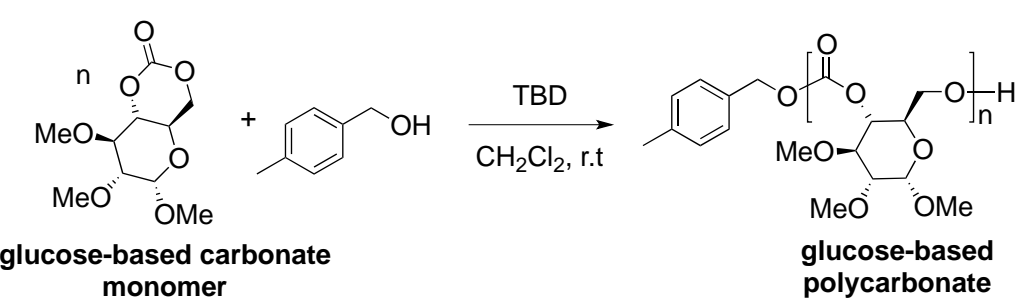

Scheme 45. TBD-catalyzed ROP of a glucose-based monomer initiated by 4-methylbenzyl alcohol.[152, 153]

Similarly, starch-g-PTMC copolymers were synthesized by TBD-catalyzed ROP of TMC in bulk at $150^{\circ} \mathrm{C}$, in the presence of native starch granules as macroinitiators (Scheme 24).[76] However, PTMC-based homopolymers $\left(M_{n}=4,700 \mathrm{~g} \cdot \mathrm{mol}^{-1} ; \emptyset=1.24\right)$ were also detected by NMR spectroscopy, suggesting initiation by propan-1,3-diol generated from hydrolysis of TMC.

Recently, Coulembier et al. reported a $\mathrm{CO}_{2}$-based switchable "on/off" ROP of $\varepsilon$-CL, using a mixture of TBD/DBU (10/1) as catalytic system and $\mathrm{BnOH}$ as initiator (Scheme 46).[154] The ROP of $\varepsilon-C L$ was performed in toluene at r.t., yielding PCL's of molar masses $M_{n}=1,400-37,000 \mathrm{~g} \cdot \mathrm{mol}^{-1}(\Theta=1.1-1.8)$. While TBD alone was active toward the ROP of $\varepsilon-C L$, DBU was inactive, but the association of both TBD and DBU resulted in the formation of a hydrogen-bond based-complex (see insert in Scheme 46) that allowed polymerizing this monomer by tempering the reactivity of the TBD catalyst. The switchable "on/off" behavior between active and dormant propagating species was demonstrated by repetitive cycles of $\mathrm{CO}_{2} / \mathrm{N}_{2}$ additions and eliminations, where the addition of $\mathrm{CO}_{2}$ resulted in a dormant carbonate "off" state. In the same manner, the TBD-catalyzed ROP of TMC could also be activated and deactivated on demand by the reversible fixation of $\mathrm{CO}_{2}$.

$\mathrm{n}$

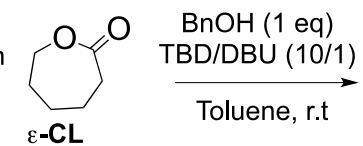

$\mathrm{H}$-bond based-complex between DBU and TBD

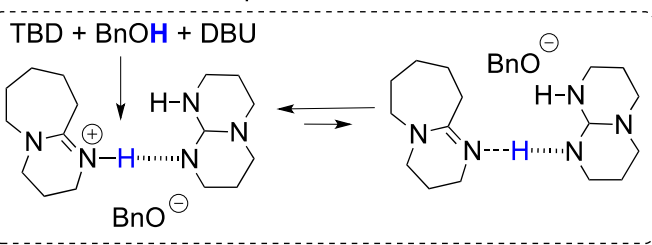

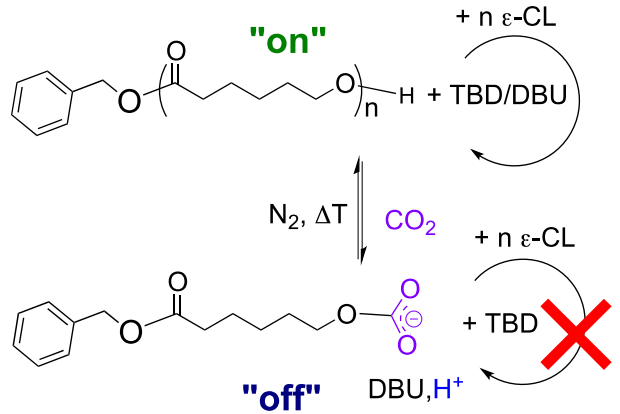


Scheme 46. $\mathrm{CO}_{2}$-based switchable ROP of $\mathrm{CL}$ using TBD/DBU (10/1) catalytic mixture with $\mathrm{BnOH}$ as initiator.[154]

There are also some examples of step-growth polymerization reactions utilizing guanidines as organocatalysts.[20,155] A series of cyclic guanidines was thus shown to trigger the polyaddition of oligomeric diols (PEO-600 and PTMO-650) and IPDI, in bulk at $60{ }^{\circ} \mathrm{C}$ (Scheme 47), affording PUs with $M_{n}=21,700-39,800$ g.mol-1 $(\Xi=1.33-1.89)$.[20] In contrast to NHC catalysis,[120] where traces of uretdione (dimer) and isocyanurate (trimer) were detected (see Scheme 33), the guanidine catalysis proceeded without any detectable side events, demonstrating the high selectivity of these catalysts for this reaction. The mechanism of this polyaddition was not clearly established, however, and the authors suggested two possible pathways (Scheme 47). In the nucleophilic mechanism, the addition of the guanidine onto the carbonyl of the isocyanate (AMM) is followed by the reaction of the activated zwitterionic acyl intermediate with the alcohol partner, providing the urethane linkage. However, an ACEM where the alcohol is activated by the base is also plausible.[20]

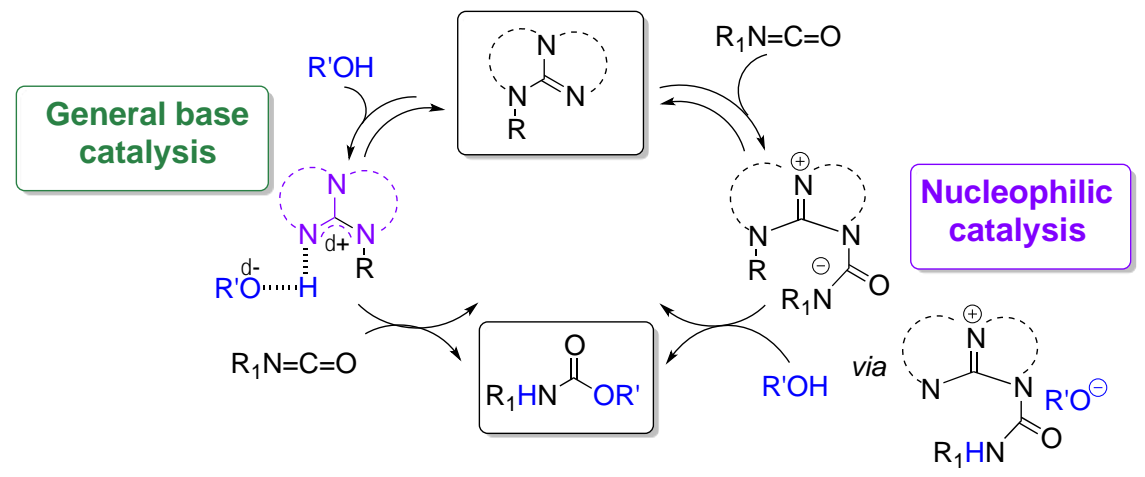

Scheme 47. Guanidine catalyzed-synthesis of PUs from IPDI and PEO.[20]

The effectiveness of DBU for synthesizing PUs was further demonstrated with the work of Hedrick, Yang et al., where PUs based on the polyaddition of methyldiethanolamine and IPDI, in presence or not of PEO, were prepared in dichloromethane at r.t.[155] Those polymers were next used to decorate silica nanoparticles, following a "grafting onto » approach. Upon quaternization of the amino groups in the main chain polymer, an antibacterial poly(ammonium)type coating was generated.

In the context of a sustainable synthesis of PUs starting from non-isocyanate-based monomers,[156] the organo-catalyzed aminolysis of cyclic carbonates, to form (poly)hydroxyurethanes (PHUs) is an interesting alternative to the isocyanate-based chemistry. PHU's of molar masses $M_{n}=12,400-53,400 \mathrm{~g} \cdot \mathrm{mol}^{-1}$ and $\Theta=1.18-1.38$ were thus obtained by step-growth polymerization of the difunctional cyclic carbonate (4-(phenoxymethyl)-1,3dioxolan-2-one derivative (B5CC) and diamines, in DMSO at r.t., using TBD as catalyst (Scheme 48).[157] Although PHUs have distinct properties than PUs, this strategy proved interesting to avoid toxic isocyanate precursors, while using the rather poor reactivity of 5-membered ring cyclocarbonates. 

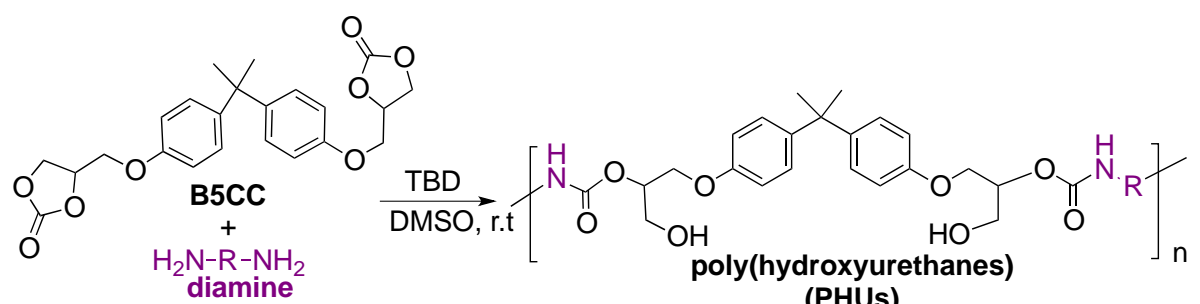

(PHUs)

Scheme 48. TBD-catalyzed step-growth polymerization between a difunctional cyclic carbonate and diamines.[157]

DBU and 1,5-diazabicyclo[4.3.0]non-5-ene (DBN) amidines, as well as the TBD guanidine, were reported by Hedrick et al. to selectively degrade poly(ethylene terephthalate) (PET), providing an attractive metal-free strategy for depolymerization of this important aromatic polyester.[158-160] Chemical degradation occurred at $150^{\circ} \mathrm{C}$, in the presence of either ethylene glycol (EG) forming bis(2-hydroxyethyl)terephthalester (BHET), or ethylene diamine (EDA) generating bis(2-aminoethyl)terephthalamide (BAETA) by-product. DBU proved more efficient than TBD for the glycolysis with EG, whereas the opposite trend was noted for amidation with EDA.

DFT calculations performed with methylbenzoate $(\mathrm{MB})$ as model substrate predicted that the nucleophilic addition of the activated alcohol (or amine) onto the carbonyl group of the ester was the rate-determining step. [159] Even though both TBD and DBU activated alcohols better than amines, theoretical studies suggested that transesterification and amidation reactions were kinetically comparable. From a thermodynamic standpoint, however, the transesterification was thermo-neutral (reversible) while the amidification was exergonic, hence, the former process was likely to revert to the starting materials.

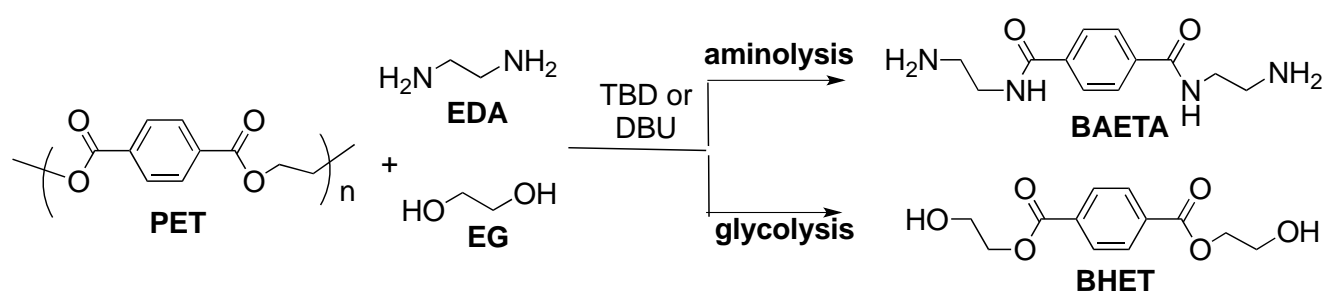

Scheme 49. Depolymerization of PET using TBD or DBU catalysis. [159, 160]

In summary, among nitrogen-containing bases, amidines and guanidines can efficiently catalyze the ROP of cyclic esters, cyclic carbonates and phospholanes, as well as the depolymerization of PET. While the DBU amidine proves to only activate the initiating alcohol, TBD can behave as a bifunctional activator for both the monomer and the alcohol. In contrast, only a few examples of amine-catalyzed ROPs (i.e. lactide and cyclic carbonates) have been reported, presumably due to their lower basicity compared to those of amidines and guanidines. The variety and commercial availability of these nitrogen-containing catalysts, combined with their ability to promote control over the polymerization reactions under mild conditions, are key advantages that make them attractive catalytic options for polymer synthesis. 


\section{Mono- or bicomponent dual catalytic systems}

One of the simplest strategies to provide simultaneous activation of both the monomer and the initiator/chain-end is to combine a weak Brønsted acid $(A)$ with a weak Brønsted base (B) in a bicomponent catalytic system. However, ambiphilic properties can be also found in a single -monocomponent- molecule, providing a more practical reaction set-up. Generally, such a dual activation is based on the formation of two different $\mathrm{H}$-bound complexes (Figure 16): a Brønsted acid-monomer (complex 1), and a Brønsted base-initiator (or growing chain; complex 2). For this purpose, some of the organocatalysts previously described (e.g. amidines, amines and phosphoric acids) can be employed.

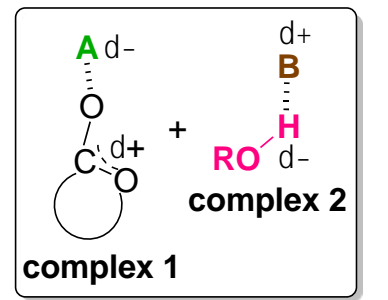

$$
\text { A }+ \text { B } \quad \text { vs }
$$

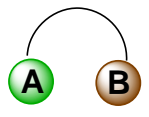

bicomponent monocomponent

system system

Figure 16. Unimolecular or bimolecular dual activation based on $\mathrm{H}$-bonding interactions.

In a recent review, Bibal and Thomas highlighted the scope of $\mathrm{H}$-bondings organocatalysts for ROP, and discussed the structure-catalytic activity relationships.[13] Here we briefly describe recent ROP examples, based on such a dual activation.

Urea and thiourea (TU) derivatives are well known to activate carbonyl-containing substrates through hydrogen-bonding.[161] Such catalysts have also been largely applied to induce the ROP of cyclic esters and carbonates, mainly in association with amines and amidines (Figure 18).[7, 14]

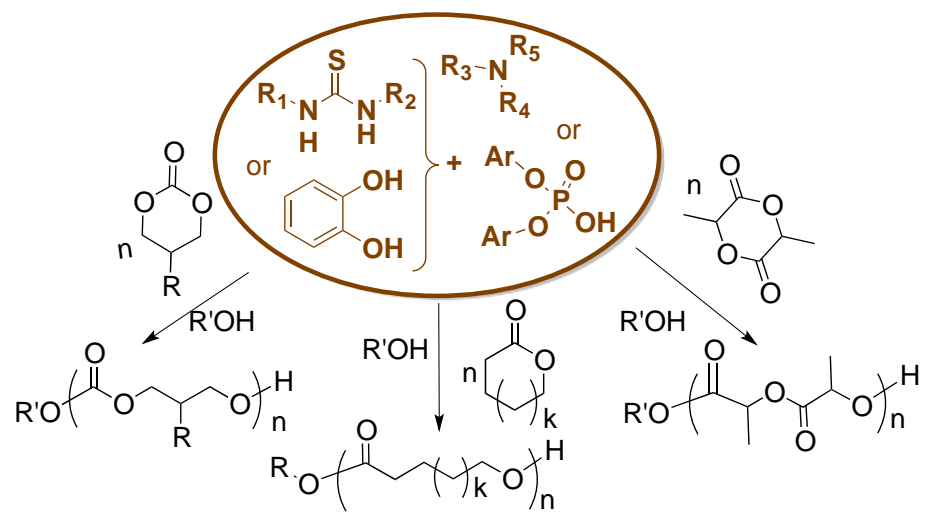

Figure 17. Overview of ROP induced by a dual-activation. 


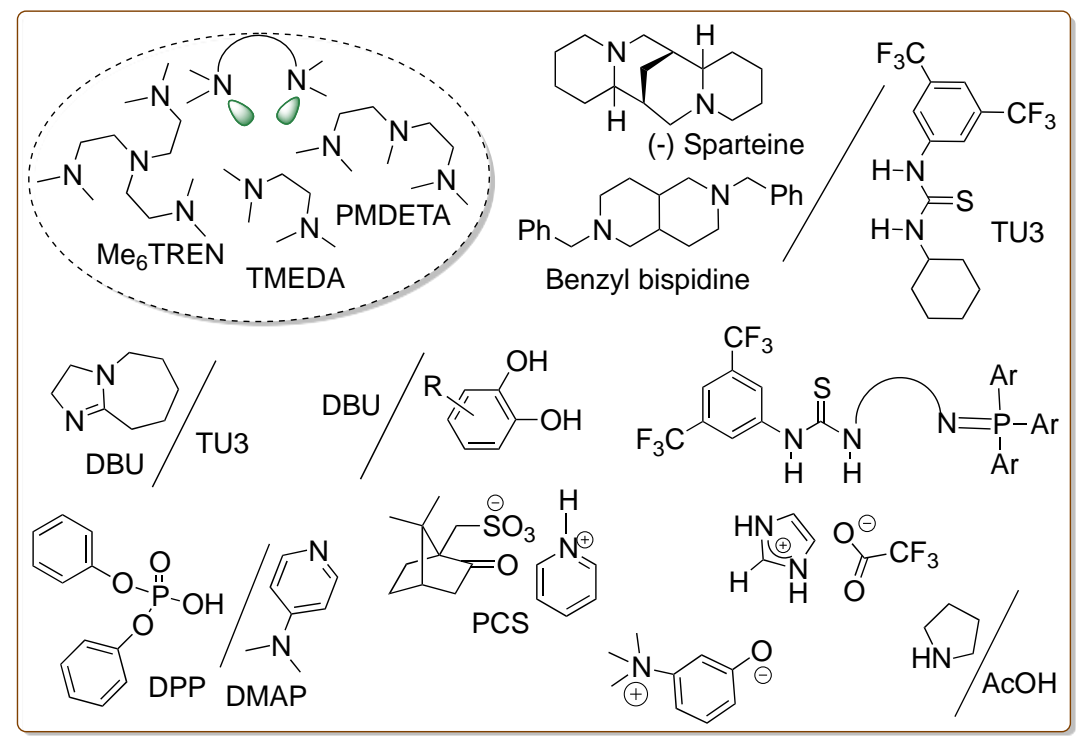

Figure 18. Representative organic polymerization catalysts inducing a dual activation.

Fig slightly modified

A series of commercially available amines, namely, TMEDA, PMDETA and tris[2(dimethylamino)ethyl]amine (Me 6 TREN) (see Figure 18$)$ combined with (N-(3,5bis(trifluoromethyl)phenyl)- $N$-cyclohexyl-thiourea (TU3; 5eq), were screened as catalytic systems for the ROP of L-LA in dichloromethane at r.t., using $\mathrm{PyBuOH}$ as initiator (Scheme 50).[133] Among the various amines investigated, $\mathrm{Me}_{6}$ TREN was found the most efficient, yielding PLLA's of molar masses $M_{n}=23,000 \mathrm{~g} \cdot \mathrm{mol}^{-1}$ and $Ð=1.06$.

The specificity of $\mathrm{Me}_{6}$ TREN was proposed to arise from the presence of multiple basic sites and the locked spatial geometry of the catalyst, allowing for a better alcohol activation through chelative effect (see insert in Scheme 50).

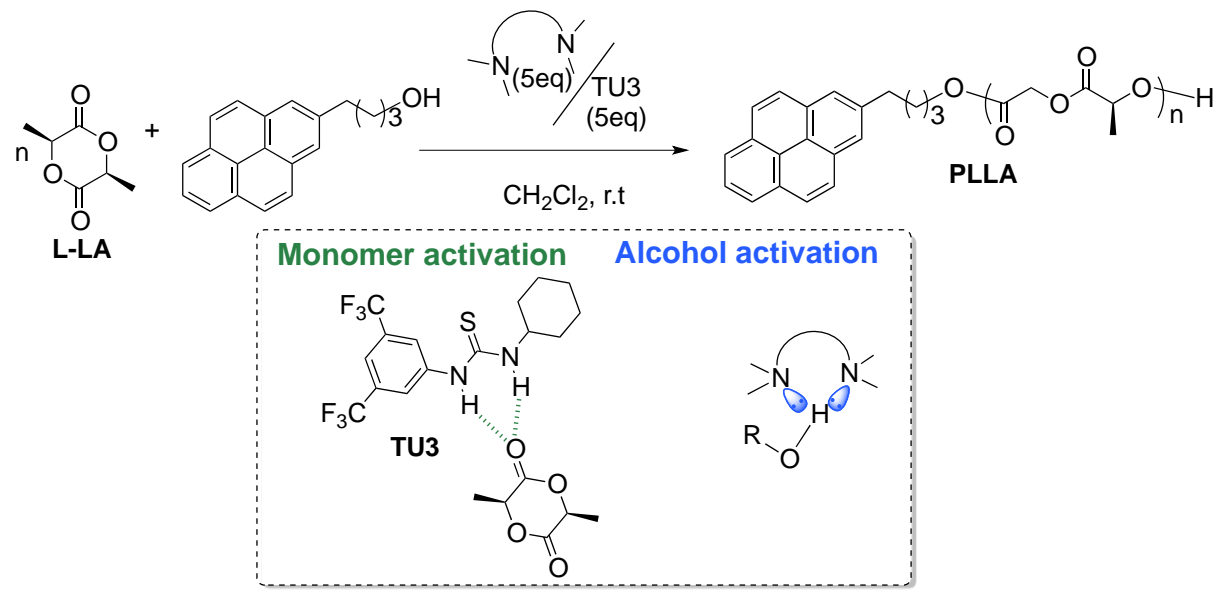

Scheme 50. ROP of L-LA using TU/amine catalytic system in the presence of pyrenebutanol.[133]

The ability of (-)sparteine/TU or DBU/TU catalytic systems for the ROP of functionalized cyclic carbonates, using alcohol initiators, has been investigated in detail by Dove et al. [147][162-165] and by Hedrick et al. [166, 167] For instance, the ROP of 5-methyl-5allyloxycarbonyl-1,3-dioxan-2-one (MAC) was successfully achieved in chloroform at r.t., in presence of $\mathrm{BnOH}$ as initiator and (-)sparteine/TU3 as organocatalyst, leading to PMAC's of 
molar masses $M_{n}=4,600-10,500 \mathrm{~g} \mathrm{~mol}^{-1}$ and $\Theta=1.12-1.49$ (Scheme 51a).[168] Block copolymers based on PMAC and PLA $\left(M_{n}=4,300-7,100 \mathrm{~g} \mathrm{~mol}^{-1} ; \oplus=1.17-1.28\right)$ were also synthesized, either by ROP of LA from a PMAC prepolymer in a one-pot process, or by ROP of MAC using a PLA macroinitiator. Copolymerization of MAC with 5-methyl-5-ethyloxycarbonyl1,3-dioxane-2-one (MTC-Et) was also reported under the same conditions, producing allyl-

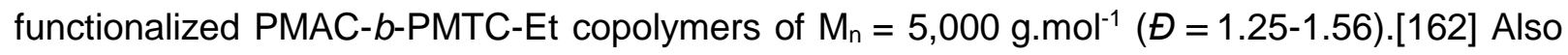
under the same conditions, dibenzyl-functional bispidine, in combination with TU3 co-catalyst, achieved rates almost identical to those observed using (-)sparteine for the ROP of TMC and MAC.[163] The polymerization was well-controlled, leading to polycarbonates of molar masses $M n=6,100-9,100$ g.mol-1 and $\Xi=1.03-1.31$, establishing that benzyl bispidine is an excellent substitute of (-)sparteine that becomes difficult to obtain commercially.

The (-)sparteine/TU3-catalyzed ROP of acrylamide-functionalized cyclic carbonates in dichloromethane at r.t. gave well-defined tert-butylacrylamide-functionalized homo- and copolycarbonates of $M_{n}=4,600-5,100 \mathrm{~g} \cdot \mathrm{mol}^{-1}$ and $D=1.12-1.32$ (Scheme 51b).[166] With the same catalytic system, the ROP of 5-methyl-5-propargyloxycarbonyl-1,3-dioxan-2-one (MPC) afforded PMPC's of molar masses $M_{n}=6,800 \mathrm{~g} \cdot \mathrm{mol}^{-1}(\Theta=1.18)$ in chloroform at r.t.[164] However, the polymerization of this monomer could be improved by switching to a DBU/TU3 catalysis. In the latter case, a PMPC of $M_{n}=16,500 \mathrm{~g} \cdot \mathrm{mol}^{-1}(\Theta=1.11)$ was obtained. The synthesis of telechelic PMPC's, poly(ether)-b-poly(carbonate)s and poly(ester)- $b$ poly(carbonate)s copolymers was also reported by using various hydroxyl-functional initiators, including disulfides, diols and MeOPEO-OH, as well as by copolymerization with L-LA.[164] Post-polymerization modification of these polymers by "thiol-yne click-chemistry" allowed accessing a range of functional polycarbonates.

a)

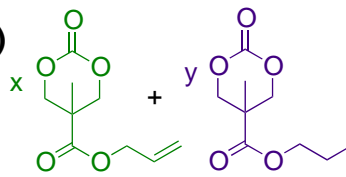

MAC

MTC-Et

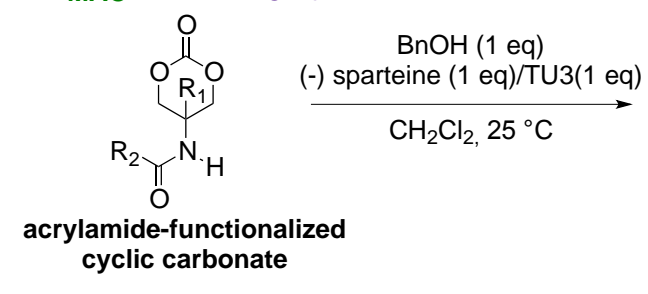

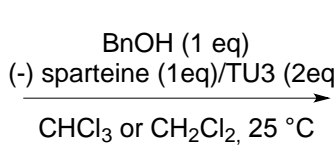

$\mathrm{CHCl}_{3}$ or $\mathrm{CH}_{2} \mathrm{Cl}_{2}, 25^{\circ} \mathrm{C}$

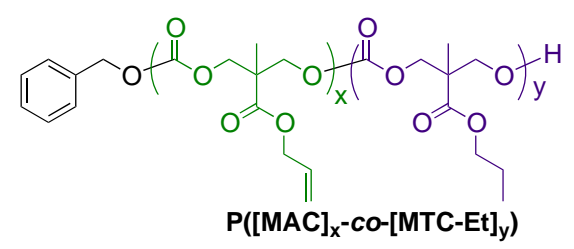

$\left.P\left([M A C]_{x}-\text { Co-[MTC-Et }\right]_{y}\right)$

b)

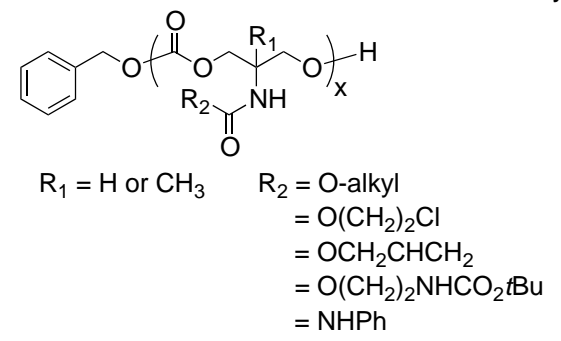

Scheme 51. Synthesis of functional polycarbonates using (-)sparteine/TU3 as catalyst and $\mathrm{BnOH}$ as initiator.[162, 166, 168]

In the same manner, the $\mathrm{DBU} / \mathrm{TU} 3$ system served to catalyze the $\mathrm{BnOH}$-initiated ROP of carbonates, including benzyl-MTC (MTC-Bn), quinine-MTC (MTC-Q) and Boc-MTC (MTCbocG), in dichloromethane at r.t. Polycarbonates of molar masses, $M_{n}=2,400-7,300 \mathrm{~g} \cdot \mathrm{mol}^{-1}$ $(\Theta<1.3)$, in agreement with values predicted from the initial monomer-to-initiator ratio, were 
thus synthesized.[167] Copolymerization of MTC-Q with MTC-bocG afforded, after deprotection, water-soluble cationic poly[(MTC-Q)-b-(MTC-G)] copolymers $\left(M_{n}=3,900 \mathrm{~g}^{\mathrm{mol}}{ }^{-1} ; \bigoplus=1.5\right)$, functionalized with both quinine and pendant guanidinium groups (Scheme 52).

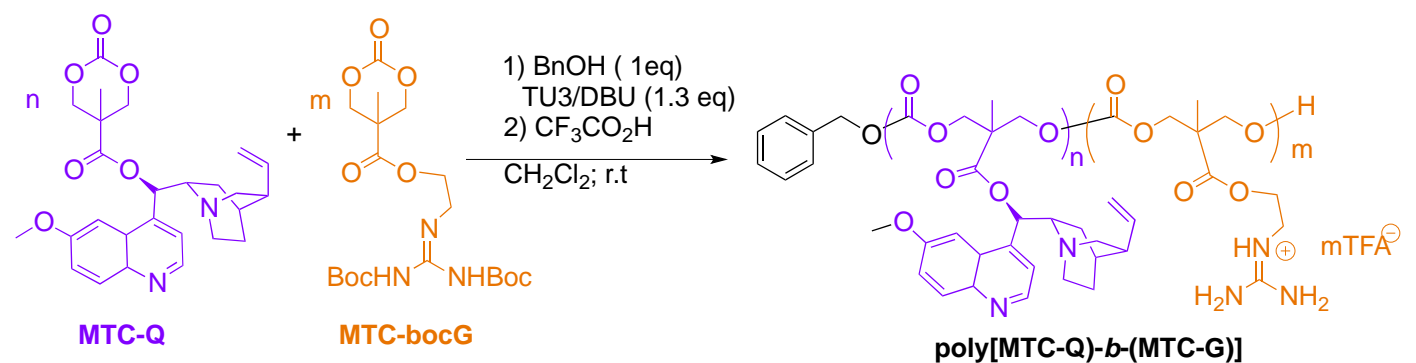

Scheme 52. DBU/TU-catalyzed synthesis of (co) polymers bearing both quinine and guanidinium groups.[167]

The benzyl-protected dihydroxylated bicyclic carbonate, namely, 9-phenyl-2,4,8,10 tetraoxaspiro[5,5]undecan-3-one (PTO), was readily polymerized in dichloromethane at r.t., using 1,4-butanediol, MeO-PEO-OH or HO-PCL-OH as (macro)initiator and DBU/TU3 as catalytic system (Scheme 53).[165, 169] A PEO-b-poly(PTO) copolymer of controlled molar mass, $M_{n}=6,300$ g.mol- ${ }^{-1}$ and $\Theta=1.06$, was obtained using MeOPEO-OH as macroinitiator. Sequential copolymerization with D,L-lactide provided an amphiphilic functional PEO- $b$ polyester-polycarbonate $\left(M_{n}=12,000 \mathrm{~g} \cdot \mathrm{mol}^{-1} ; \boxminus=1.07\right)$. The physicochemical properties of these copolymers could be tuned by optimized post-modification, enabling their potential use as biodegradable materials.

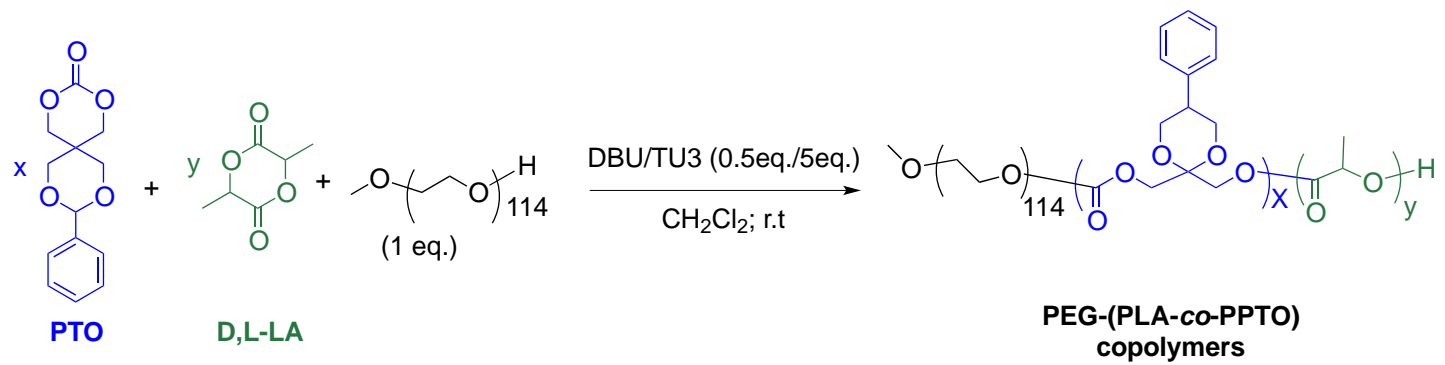

Scheme 53. Synthesis of amphiphilic PEO-poly(ester carbonate) using DBU/TU3 catalysis.[169]

Waymouth et al. reported the ROP of four $N$-substituted morpholin-2-ones, namely, decyl 2-oxomorpholine-4-carboxylate (MoDec), N-benzyl morpholine-2-one $\left(\mathrm{M}_{\mathrm{B} n}\right), \quad N$-phenyl morpholine-2-one $\left(\mathrm{MPh}_{\mathrm{Ph}}\right)$ and $\mathrm{N}$-butyl morpholine-2-one $\left(\mathrm{M}_{\mathrm{Boc}}\right)$, to generate a new class of functionalized poly(aminoesters). PyBuOH was used as initiator and TBD or DBU/TU3 as catalytic systems (0.5 - 2 mol\% relative to the initiator).[170] Polymerizations were carried out in toluene at $25^{\circ} \mathrm{C}$ and, among the series, only $M_{O D e c}$ and $M_{B o c}$ provided functionalized poly(aminoesters) of molar masses $M_{n}=10,800-22,600$ g.mol-1 ( $\left.~=1.07-1.46\right)$. In contrast, $M_{\mathrm{Bn}}$ and $\mathrm{M}_{\mathrm{Ph}}$ did not polymerize at all under similar conditions. DFT calculations revealed that $\mathrm{N}$-atoms of the morpholin-2-ones $\mathrm{M}_{\mathrm{Bn}}$ and $\mathrm{M}_{\mathrm{Ph}}$ were pyramidalized and thus less susceptible to 
ring-opening, whereas those of the $\mathrm{M}_{\mathrm{ODec}}$ and $\mathrm{M}_{\mathrm{Boc}}$ were typical of planar amides, favoring the ring-opening.[170]

$$
\begin{array}{ll}
\text { Toluene, r.t } & \text { RBU/TU }
\end{array}
$$

Scheme 54. Polymerization of morpholin-2-ones with $\mathrm{ROH}$ initiator and catalyzed by

$$
\text { DBU/TU3.[170] }
$$

Phenol derivatives represent another class of small molecules with $\mathrm{H}$-bond donor capabilities and that can be involved in polymerization reactions, by analogy with the activation of monomer by thiourea derivatives.

Combining DBU and phenol thus proved suitable to catalyze the ROP of $\delta$-VL and $\varepsilon-C L$, with 4-biphenylmethanol (BPM) as initiator (Scheme 55).[171] Reactions were performed in dichloromethane at r.t., under bench conditions (no pre-drying of reactants, $4 \AA$ molecular sieves). Quantitative conversion of $\delta$-VL was obtained after $24 \mathrm{~h}$, leading to PVL's of controlled molar masses $M_{n}=1,200-6,900$ g. mol-1, and narrow dispersities $\triangleq=1.08-1.12$. In contrast the $\mathrm{DBU} /$ phenol-catalyzed ROP of $\varepsilon-\mathrm{CL}$ required longer reaction times ( $48 \mathrm{~h}$ and $120 \mathrm{~h}$ ), and reached only $57-79 \%$ of conversion. This lower reactivity of $\varepsilon$-CL compared to $\delta$-VL, in presence of $\mathrm{H}$-bonding catalysts, was attributed to the occurrence of transesterification in the case of $\varepsilon$ CL.[135] Using this strategy, block copolyesters PVL-b-PLA and PCL-b-PLA were successfully prepared. A supramolecular mechanism involving formation of $\mathrm{H}$-bond phenol:monomer and DBU:initiator (or chain-end) complexes was proposed (see insert in Scheme 55). Compared to the DBU/TU catalytic system, DBU/phenol has the practical advantage that phenol derivatives are commercially available, operating at r.t under mild experimental conditions.
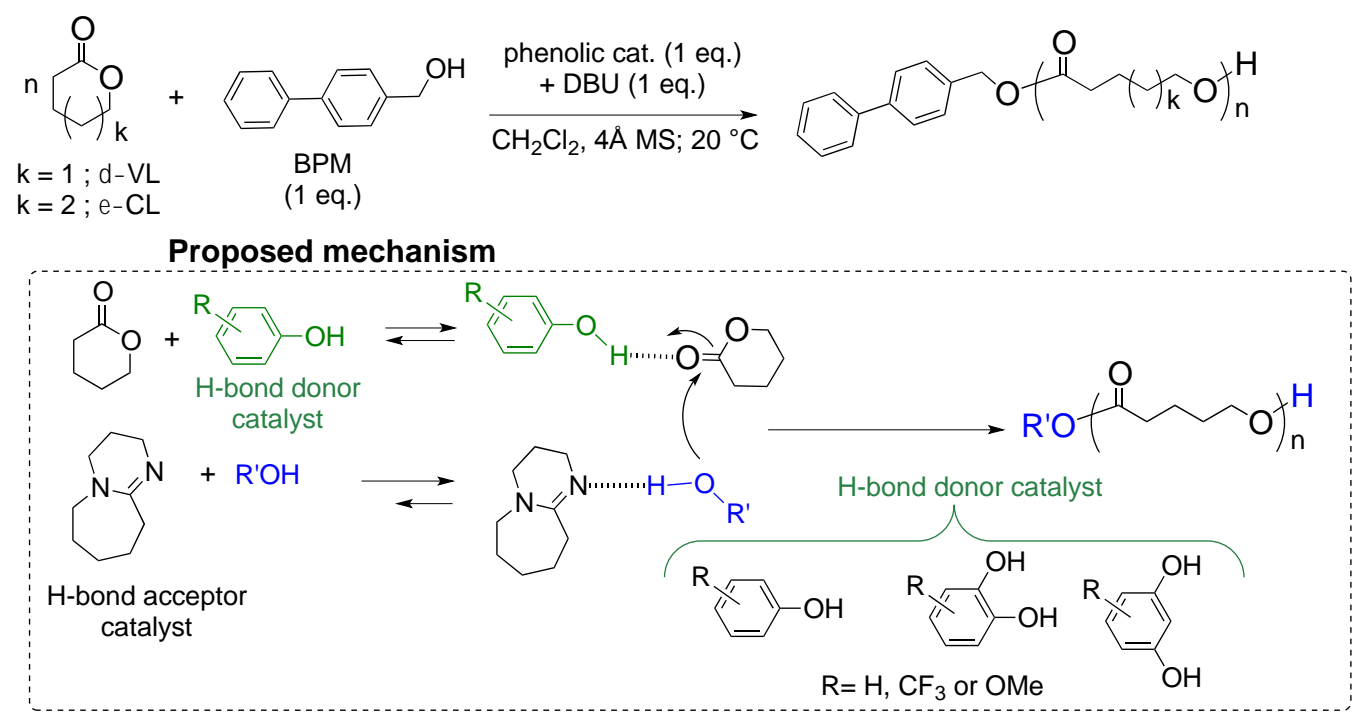
Scheme 55. DBU/phenol-catalyzed ROP of $\delta$-VL and $\varepsilon-C L$ in presence of an alcohol initiator.[171]

The stoichiometric combination of benzoic acid (BA) and DBU was investigated as catalyst by Hedrick et al. for the polymerization of L-LA with $\mathrm{BnOH}$ as initiator in dichloromethane at r.t. [172] Despite the formation of the corresponding salt in solution, this combination allowed polymerizing L-LA in a controlled fashion in dichloromethane, at r.t.. Computational analysis suggested a bifunctional catalytic mechanism wherein both the monomer and propagating hydroxyl group were activated by the amidinium carboxylate catalyst.

Kakuchi et al. combined DMAP (6 eq. relative to the initiator) and diphenyl phosphate (DPP; 3 eq.) to induce the ROP of $\varepsilon-C L$ and L-LA in dichloromethane at r.t., in presence of 3phenyl-1-propanol (PPA) as initiator ( Scheme 56).[173] DMAP was added as co-catalyst because DPP alone was inactive. This led to a well-defined PLLA of $M_{n}=19,200 \mathrm{~g} \cdot \mathrm{mol}^{-1}$ and $\Xi=1.10$. Furthermore, this combination allowed synthesizing various copolyesters, including, PVL-b-PLLA $\left(M_{n}=11,600 \mathrm{~g} \cdot \mathrm{mol}^{-1} ; \quad \nexists=1.16\right)$, PCL-b-PLLA $\left(\mathrm{M}_{\mathrm{n}}=12,100 \mathrm{~g} \cdot \mathrm{mol}^{-1} ; \quad \Xi=1.12\right)$, PDXO-b-PLLA $\left(M_{n}=12,100 \mathrm{~g} \cdot \mathrm{mol}^{-1} ; \quad \Xi=1.08\right)$ and PTMC- $b$-PLLA $\left(M_{n}=11,000 \mathrm{~g} \cdot \mathrm{mol}^{-1}\right.$; $\bigoplus=1.1$ ) diblock copolymers.

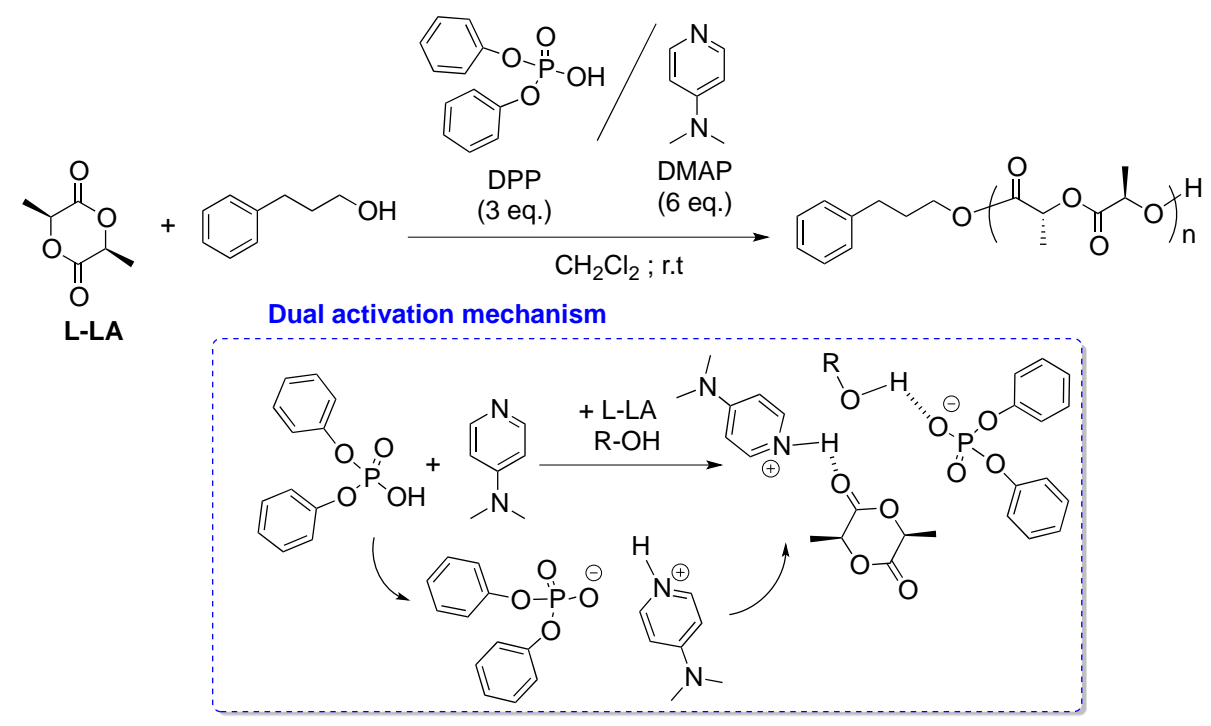

Scheme 56. Polymerization of L-LA using DPP/DMAP catalyst in presence of alcohol initiator.[173]

In the same manner, Zinck et al. triggered the ROP of rac-LA using DMAP combined with acid-base salts, such as $(R)-(1)$-binaphthyl-diyl hydrogen phosphate (BNPH)/DBU and (1 $R$ )-(-)10-camphorsulfonic acid (CSA)/DMAP as catalytic systems, and $\mathrm{BnOH}$ as initiator in dichloromethane at $35^{\circ} \mathrm{C}$.[174] Well-defined PLA's of $M_{n}=2,700-10,900 \mathrm{~g} \cdot \mathrm{mol}^{-1}(\Theta=1.07$ 1.09) were obtained, although the polymerization was relatively sluggish $(70 \%$ of monomer conversion after two weeks). 


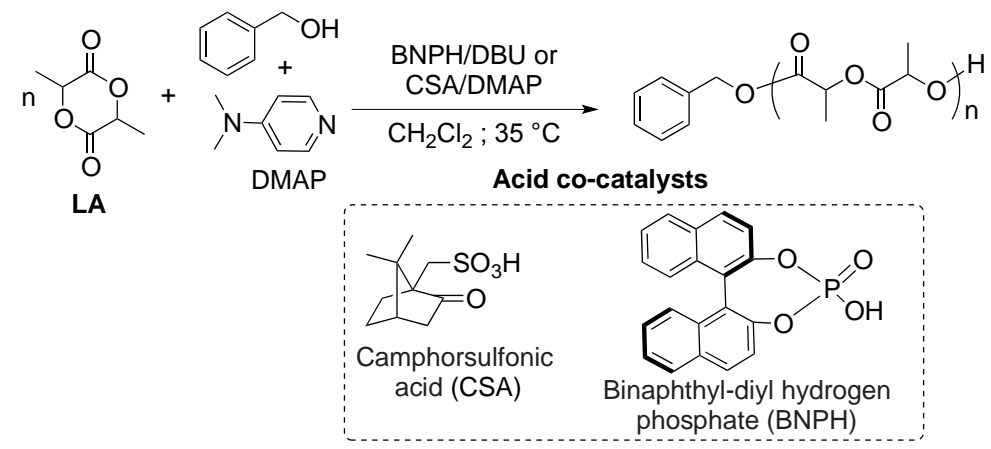

Scheme 57. ROP of LA using acid-base salts as catalytic systems in presence of DMAP/BnOH as initiating system.[174]

Later on, Peruch et al. associated DMAP to TfOH or MSA ( 0.5 eq.) as a catalytic system for the ROP of L-LA, $\varepsilon-\mathrm{CL}$ and $\delta$-VL initiated by alcohols, in bulk at $100^{\circ} \mathrm{C}$.[175] DMAP/DMAP-TfOH appeared as the most active catalytic system, leading to linear and/or starlike PLLA's of molar masses $M_{n}=3,200-140,000 \mathrm{~g} \cdot \mathrm{mol}^{-1}(\Theta=1.10-1.25)$ and polylactones of $\mathrm{M}_{\mathrm{n}}=1,800-10,800 \mathrm{~g} \cdot \mathrm{mol}^{-1}(\Theta=1.12-1.38)$. PCL- $b$-PLLA and PVL- $b$-PLLA block copolymers of $M_{n}=12,800(\theta=1.36)$ and $14,300 \mathrm{~g} \cdot \mathrm{mol}^{-1} \quad(\theta=1.16)$, respectively, were successfully synthesized through sequential polymerization, with lactones being first polymerized.

An ionic catalyst with the cation and the anion activating both the monomer and the $\mathrm{ROH}$ initiator has also been described by Coulembier et al.[176, 177] More specifically, an imidazolium trifluoroacetate salt was employed for the controlled ROP of L-LA, using BnOH as initiator (Scheme 58).[176] A PLLA of molar mass $M_{n}=8,700 \mathrm{~g} \cdot \mathrm{mol}^{-1}(\Theta=1.28)$ was rapidly obtained after $3.7 \mathrm{~h}$ of reaction in bulk at $140^{\circ} \mathrm{C}(84 \%$ of monomer conversion). Polymerization proceeded by a bifunctional mechanism resembling that operating with lipase B (produced by the yeast Candida antartica), where the imidazolium activates the carbonyl moiety through $\mathrm{H}$ bonding, while the trifluorocarboxylate interacts with the initiating/propagating alcohol (see insert in Scheme 58).

In the same manner meta-(trimethylammonio)phenolate betaine proved efficient catalyst for the ROP of L-LA, $\varepsilon-C L$ and TMC in chloroform at $21^{\circ} \mathrm{C}$ using 1-pyrenemethanol as initiator, leading to polymers of $M_{n}=12,300-48,000 \mathrm{~g} \cdot \mathrm{mol}^{-1}(\Theta=1.04-1.47) \cdot[177] \mathrm{In}$ this case, the ammonium betaine was proposed to activate the initiating and propagating alcohol site, allowing for a rapid and controlled polymerization. 


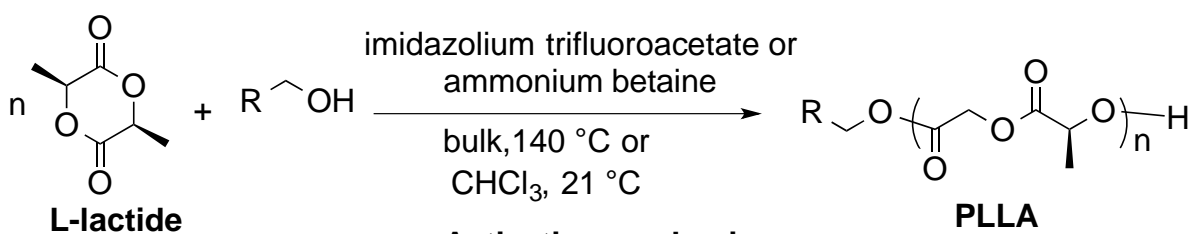

L-lactide

Activation mechanism

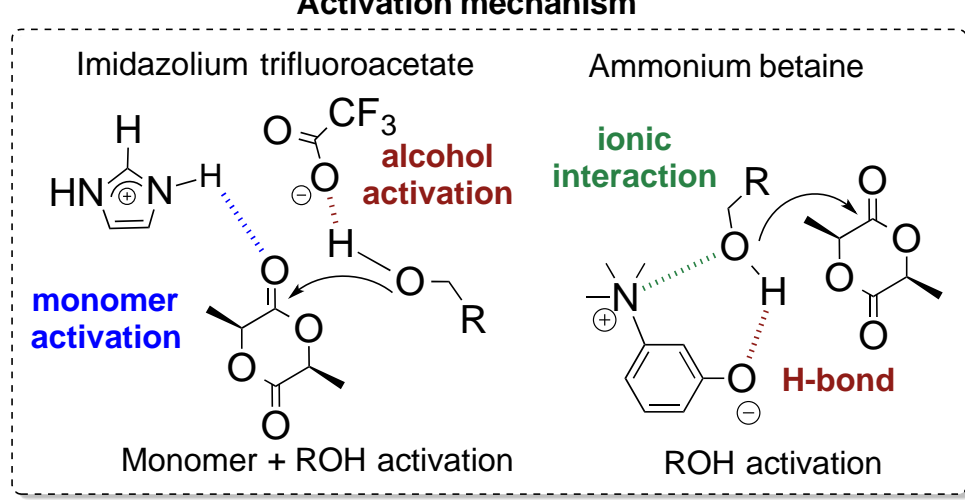

Scheme 58. ROP of L-LA using imidazolium-salt or ammonium betaine as catalyst in the presence of $\mathrm{ROH}$. [176, 177] Scheme modified

The ROP of TMC was also investigated using an acid-base binary organocatalytic system composed of the guanidine MTBD and the acid $\mathrm{TfOH}$, with $\mathrm{BnOH}$ as the initiator.[178] Using an optimal ratio MTBD/TfOH of 2/1, well-defined PTMC's of molar masses ranging from $2,800-14,400 \mathrm{~g}^{\mathrm{mol}}{ }^{-1}(\Theta=1.1-1.4)$ were obtained in dichloromethane at r.t.. Moreover, this MTBD/TFA catalytic system allowed synthesizing a PTMC-b-PLLA diblock copolymer of $M_{n}=13,000 \mathrm{~g} \cdot \mathrm{mol}^{-1}(E=1.2)$ upon sequential polymerization of TMC and L-LA.

Recently, Cramail, Taton et al. reported the first example of polyaldol synthesis via a stepgrowth cross-polymerization of bis-aldehydes and bis-ketones using a combination of pyrrolidine and acetic acid as catalytic system (Scheme 59).[22] After $72 \mathrm{~h}$ of reaction in THF at r.t., polyaldols of $M_{n}=6,700 \mathrm{~g} \cdot \mathrm{mol}^{-1}$ and $M_{w}=12,900 \mathrm{~g} \cdot \mathrm{mol}^{-1}(\nexists=1.9)$ could be prepared. The presence of acetic acid $(\mathrm{AcOH}, 150 \mathrm{~mol} \%$ relative to the monomers) allowed increasing the molar masses, while broadening the molar mass distribution $\left(M_{n}=11,100 \mathrm{~g} \cdot \mathrm{mol}^{-1}\right.$ and $\left.M_{w}=55,000 \mathrm{~g} \cdot \mathrm{mol}^{-1} ; \oplus=2 \cdot 6-4 \cdot 9\right)$. Analysis of as-obtained polymers by NMR spectroscopy confirmed the formation of $\beta$-keto alcohol units (polyaldols), but also evidenced the presence of $20-30 \%$ of conjugated ketones, as a result of the dehydration of $\beta$-keto alcohol units (see insert in Scheme 59).

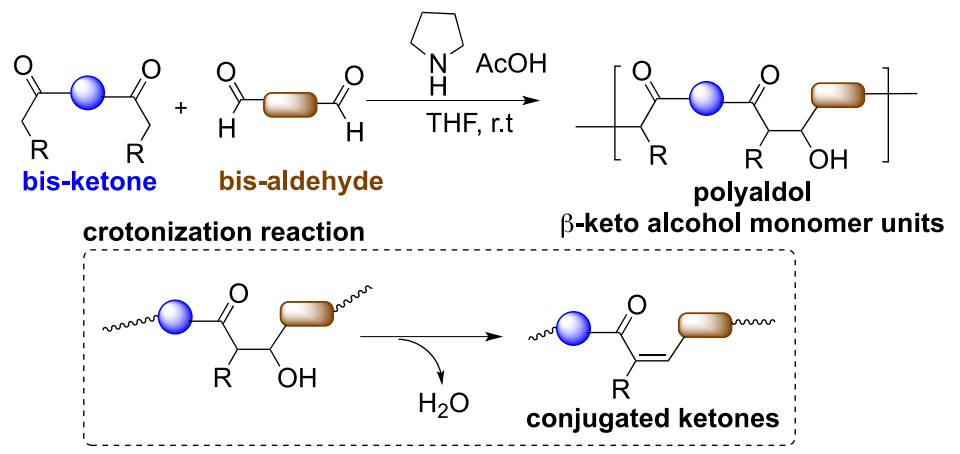


Scheme 59. Polymerization between bis(ketone)s and bis(aldehydes)s forming poly(aldol)s.[22]

The direct bulk aldol step-growth polymerization of acetaldehyde at r.t., catalyzed by proline-derived catalysts, in presence of acetic acid was also described (Scheme 60).[179] Only polyene-type (I; $\left.M_{n}=200 \mathrm{~g} \cdot \mathrm{mol}^{-1}\right)$ and polyol-type (II; $M_{n}=200-300 \mathrm{~g} \cdot \mathrm{mol}^{-1}$ and $M_{w}=800-1,100 \mathrm{~g} \cdot \mathrm{mol}^{-1}$ ) oligomers were obtained in bulk at r.t.. Poly(vinyl alcohol-coethylene)-type oligomers were subsequently prepared, under hydrogenation and deacetalization conditions.

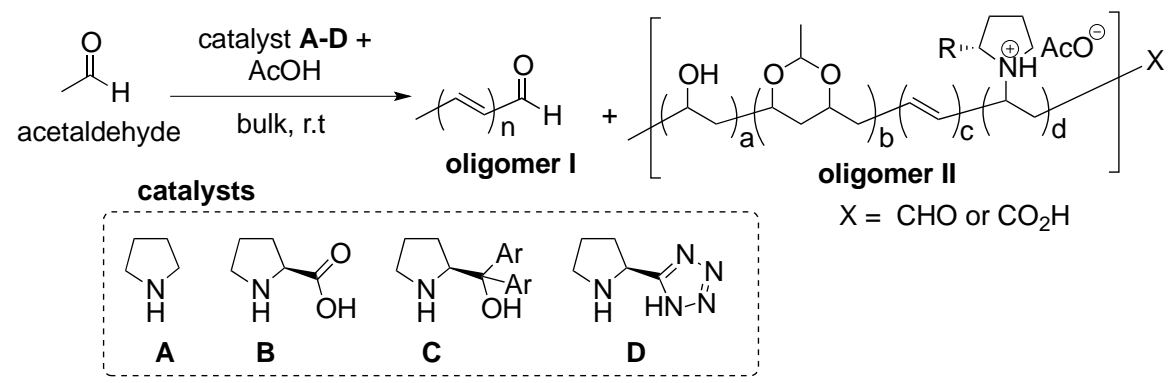

Scheme 60. Direct aldol polymerization of acetaldehyde using proline derivatives and acetic acid as catalysts. [179]

Cramail, Taton et al. also reported the synthesis of unprecedented hyperbranched polyacetals (HBPA's) with a degree of branching (DB) equal to unity, by an acid-catalyzed stepgrowth polymerization of a simple $\mathrm{AB}_{2}$ type monomer.[180] Indeed, the direct polycondensation of $p$-hydroxymethylbenzaldehyde dimethylacetal (26, Scheme 61) and its nonprotected homologue, namely, p-hydroxymethylbenzaldehyde 27, was catalyzed by pyridinium camphorsulfonic acids (PCS) in toluene or in THF at r.t.[180] While HBPA's with a DB around 0.5 were produced through polytransacetalization of monomer 26, monomer 27 led to HBPA's with $\mathrm{DB}=1$ by polyacetalization. This major difference was attributed to the reactivity of intermediate units formed during the first condensation reaction for each monomer. Upon condensation of monomer $\mathbf{2 6}$, a rather stable acetal intermediate (= linear unit) was formed in contrast to the unstable hemiacetal intermediate arising from monomer $\mathbf{2 7}$, and that readily evolved to a stable (dendritic) acetal unit after reaction with another alcohol function. Previous works by Ramakrishnan et al. had shown that hyperbranched polyacetals with $\mathrm{DB} \approx 0.5$ could be achieved using various $A B_{2}$-type hydroxymethylbenzaldehyde dialkyl acetal $(28)$.[181, 182] 


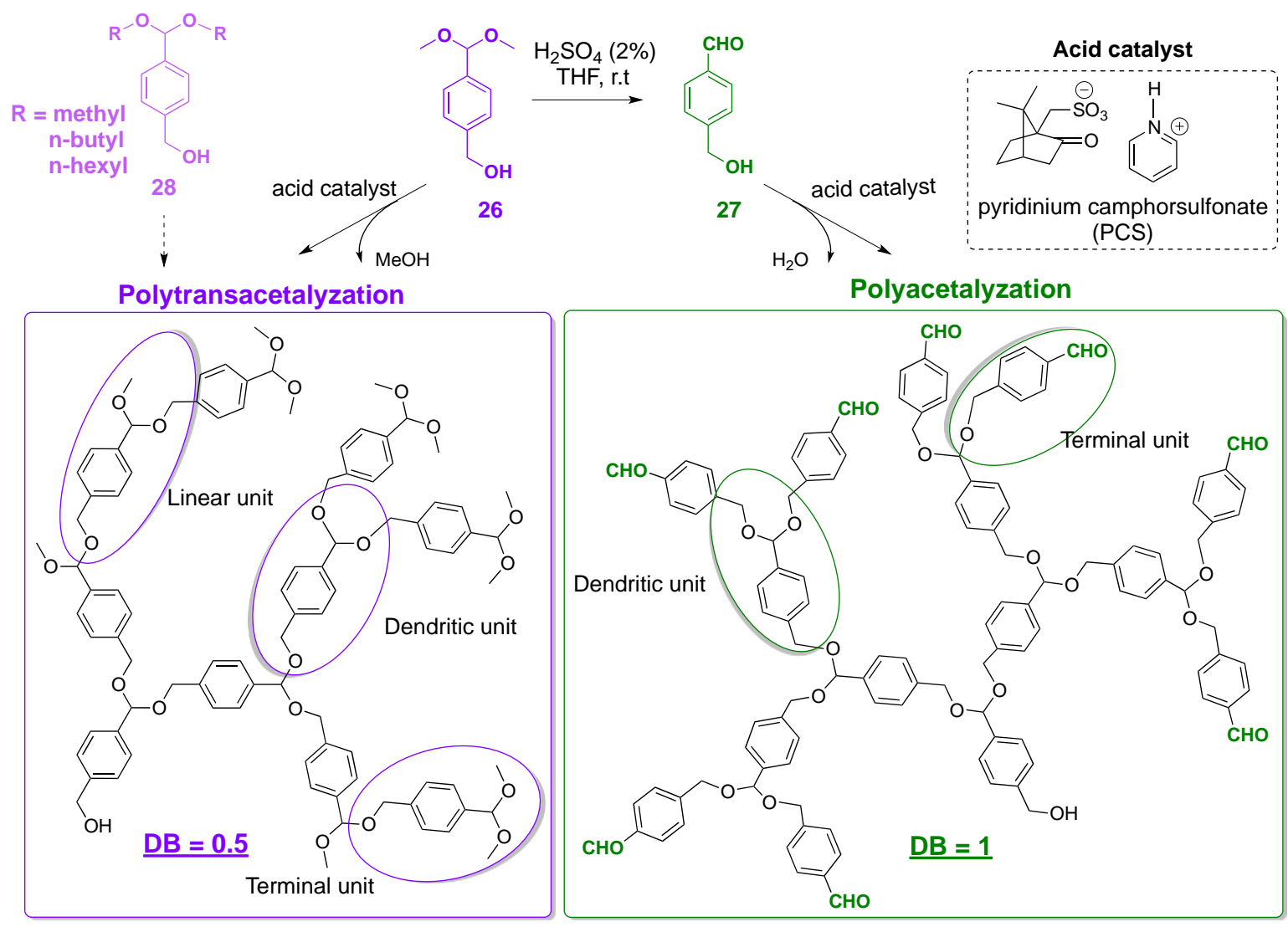

Scheme 61. Synthesis of hyperbranched polyacetals (HBPAs) via polytransacetalyzation of monomer $1(\mathrm{DB} \approx 0.5)$ and polyacetalyzation of monomer $2(\mathrm{DB}=1)$ using acid catalysts. [180]

As mentioned above, instead of using a bi-component system promoting a dual activation, bifunctionality can be provided from one single molecule. For instance, the asymmetric step-growth polymerization of prochiral bis-anhydrides with diols was investigated using by a cinchona-type organocatalyst in THF at $25^{\circ} \mathrm{C}$ (Scheme 62).[183] The reaction achieved $90 \%$ of conversion after seven days, leading to chiral polyester oligomers with a DP up to $14\left(\mathrm{M}_{\mathrm{n}}=400-8,900 \mathrm{~g} \cdot \mathrm{mol}^{-1} ; \bigoplus=1.25-2.15\right)$, and with high levels of stereocontrol ( $89 \%$ of enantiomeric excess). Such a polymerization was thought to proceed through transfer of the chirality from the cinchona alkanoïd catalyst to the growing polymer chain, with the catalyst acting as dual activator of both the alcohol and the anhydride.

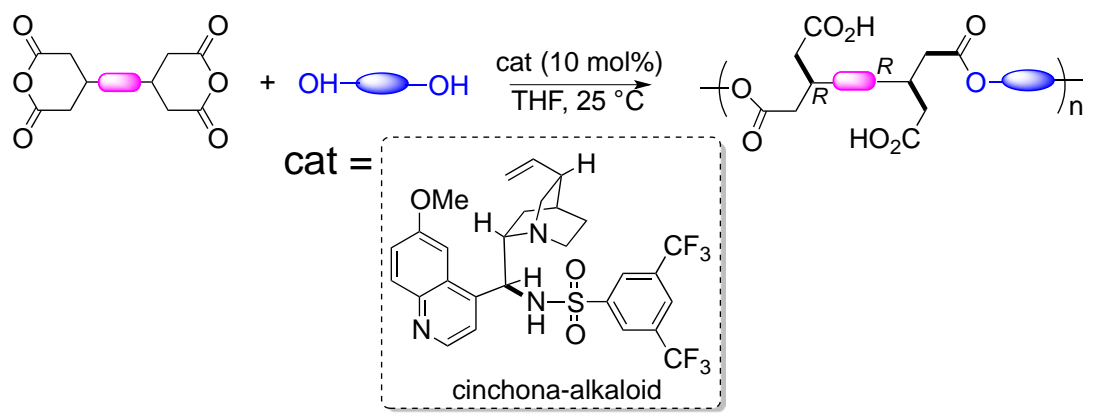

Scheme 62. Synthesis of polyesters by desymmetrizing step-growth polymerization of bisanhydrides with diols.[183] 
Triaryl iminophosphoranes featuring a thiourea moiety were reported to efficiently catalyze the ROP of L-LA, $\delta$-VL and $\varepsilon-C L$, using PyBuOH as initiator (Scheme 63).[184] Quantitative conversion was obtained within $10 \mathrm{~min}$ in dichloromethane at r.t., affording polyesters of molar masses $M_{n}=4,900(\theta=1.23), 32,900 \mathrm{~g} \cdot \mathrm{mol}^{-1}(\Theta=1.04)$ and $37,400 \mathrm{~g} \cdot \mathrm{mol}^{-1}(\Theta=1.08)$ for PCL, PLLA and PVL homopolymers, respectively. PVL- $b$-PLA and PCL- $b$-PLA diblock copolymers were also obtained by sequential addition of monomers. Importantly, mono-functional iminophosphoranes, i.e. with no thiourea moiety, did not induce any polymerization, demonstrating the crucial role of the thiourea co-catalyst for monomer activation (Scheme 63).

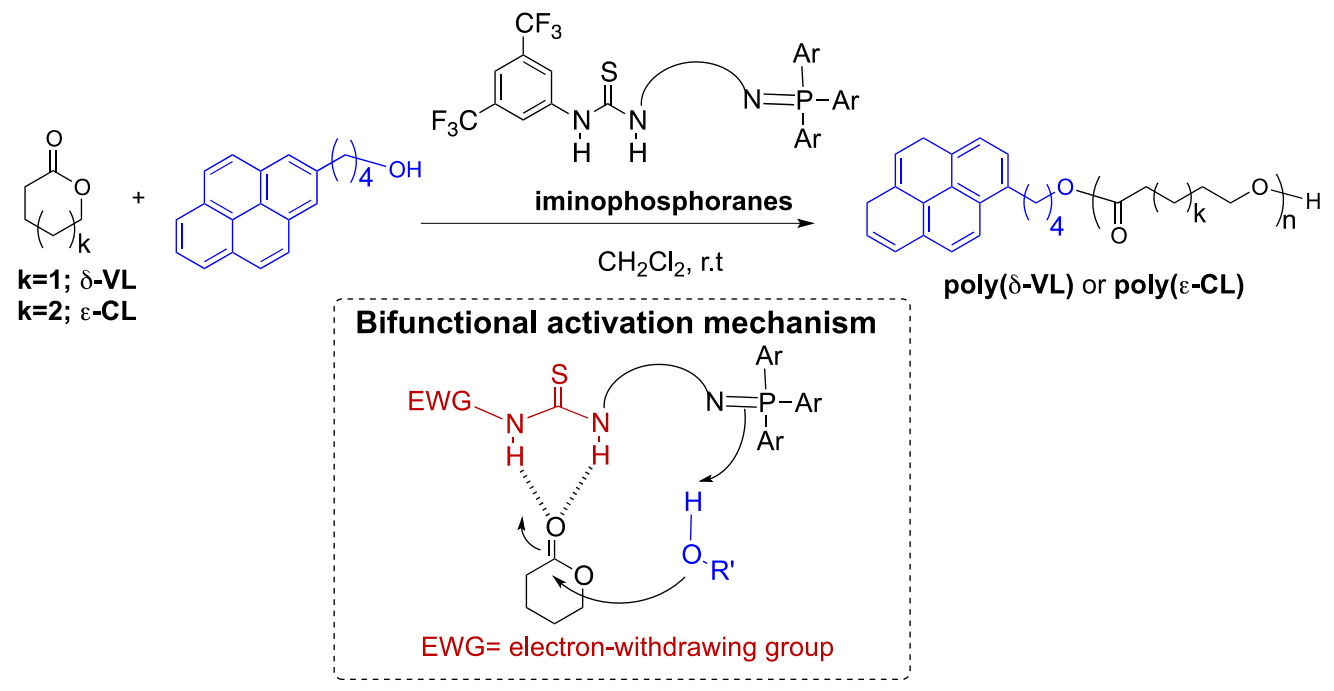

Scheme 63. ROP of cyclic esters catalyzed by iminophosphoranes in presence of alcohol.[184]

In summary, the capability of thioureas (TUs) or phenol derivatives to activate carbonyl substrates combined with the propensity of Brønsted bases to assist the deprotonation of the alcohol initiator have allowed controlling the ROP of cyclic esters and cyclic carbonates, within both mono- and bicomponent catalytic systems. Such an activation, inspired from the multiple $\mathrm{H}$-bonding motifs found in enzymatic active sites, provides high chemoselectivity, very few side reactions, such as transesterification, being observed during the polymerization.

\section{Polymerizations directly induced by organic initiators}

Here a short overview of recent polymerization reactions mediated by small organic molecules as direct initiators -and not as catalysts- will be given. Among organic activators described in the previous sections, Brønsted bases, including $\mathrm{N}$-heterocyclic carbenes (NHCs), amidines, guanines and phosphazenes, have indeed served as powerful nucleophiles to directly initiate chain-growth polymerization reactions, either through ring-opening for cyclic monomers (Scheme 64a) or through 1,4 conjugate addition for (meth)acrylic derivatives (Scheme 64b). 
a) ROP of cyclic monomers
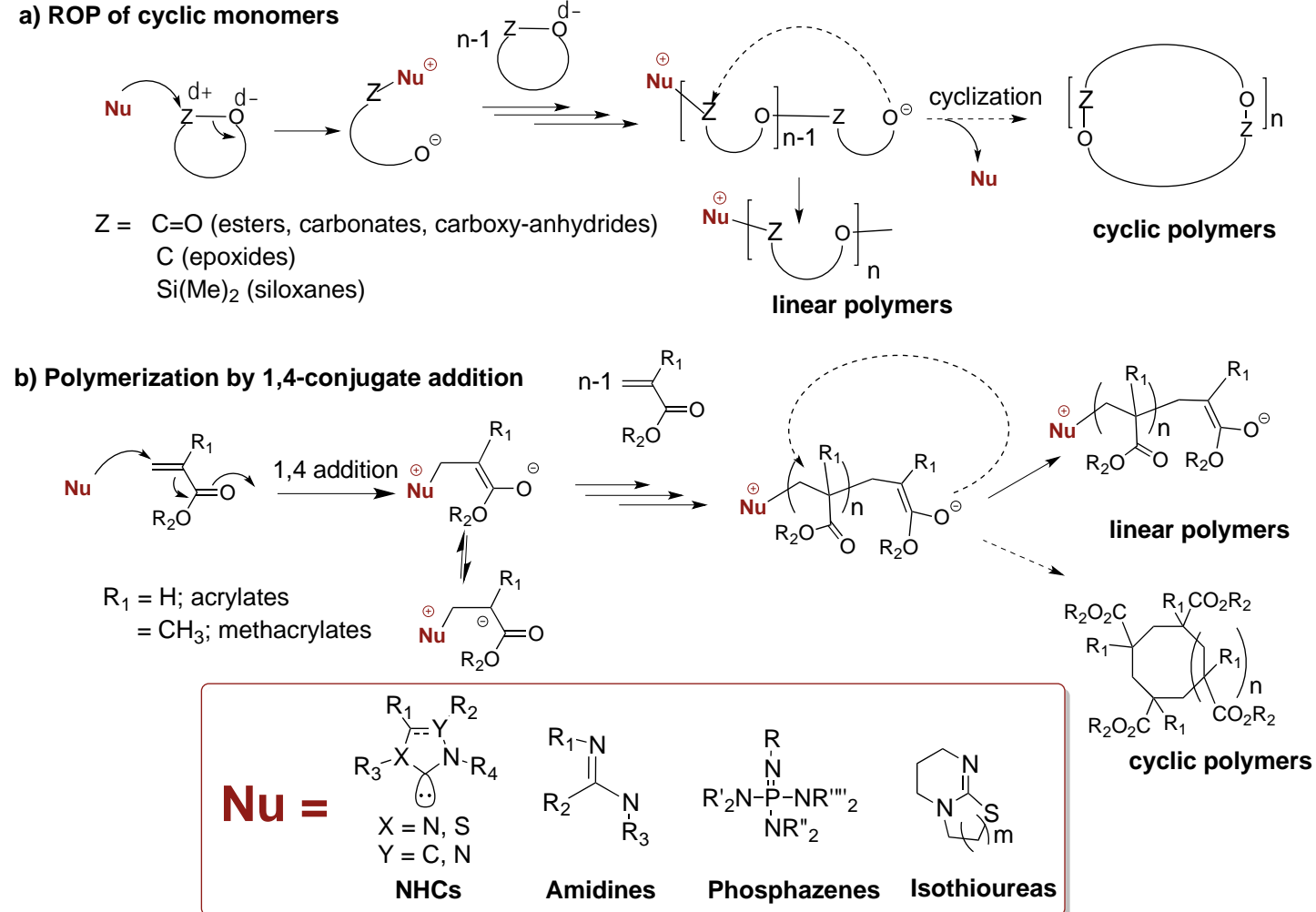

Scheme 64. Nucleophilic-induced: a) ROP; b) vinyl polymerization in absence of any co-initiator.

Hedrick, Waymouth et al. first demonstrated that the ROP of D,L-LA could be mediated by 1,3-bis(2,4,6-trimethylphenyl)imidazol-2-ylidene $\left(\mathrm{NHC}^{\mathrm{Mes}}=\mathrm{NHC}\right.$ in Figure 2) to generate cyclic PLA's with molar masses $M_{n} \leq 30,000$ g.mol${ }^{-1}$ and $\Theta=1.14-1.31 .[7,12] A$ zwitterionic ringopening polymerization (= ZROP) mechanism was put forward with the carbene playing the role of a nucleophile (Scheme 64a). The same reaction was reported later on, using methyl-3menthoxymethylimidazol-2-ylidene (NHC16 in Figure 2), also leading to cyclic PLA's but of higher molar masses $\left(M_{n}=36,500 \mathrm{~g} \cdot \mathrm{mol}^{-1}\right.$ and $\left.\Xi=1.89\right) \cdot[185]$

The ZROP of lactones by different NHCs also produced high molar mass cyclic polylactones. For instance, the ROP of $\varepsilon-\mathrm{CL}$ and $\delta$-VL in THF at r.t., from 1-isopropyl-3benzylimidazol-2-ylidene (NHC14 in Figure 2) or methyl-3-menthoxymethyl imidazol-2-ylidene (NHC16) led to cyclic homopolyesters and copolyesters, with molar mass $M_{n}=31,900 \mathrm{~g} \cdot \mathrm{mol}^{-1}$

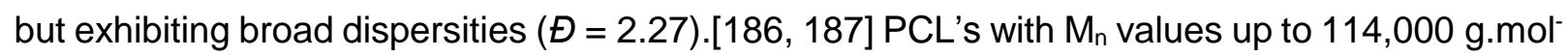
${ }^{1}$ were also synthesized in toluene at r.t., when using 1,3,4,5-tetramethylimidazol-2-ylidene (NHC18) or 1,3-bis(isopropyl-4,5-dimethyl)imidazol-2-ylidene (NHC19).[188] Molar masses were found to increase with increasing monomer conversion, but remained significantly higher than values predicted from the initial monomer-to-initiator ratio, presumably due to the occurrence of several competitive polymerization mechanisms.

Modeling experiments revealed that initiation of lactones was a slow and reversible process, while propagation was rapid (route $A$ in Scheme 65) but accompanied by several side reactions.[188, 189] These include intramolecular backbiting of alkoxide chain-ends on internal 
ester moieties of growing zwitterions 29 (route B), and intermolecular attack of active zwitterions on internal esters of other zwitterions (route C), as well as cyclized chains (route D).[188] This caused the formation of both higher molar masses linear (30 in Scheme 65) and cyclic chains (31 and 33). Attack of active zwitterionic imidazolium alkoxides on cyclized chains (route D) was proposed to be a key step, leading to high molar masses cyclic PCL's (33).

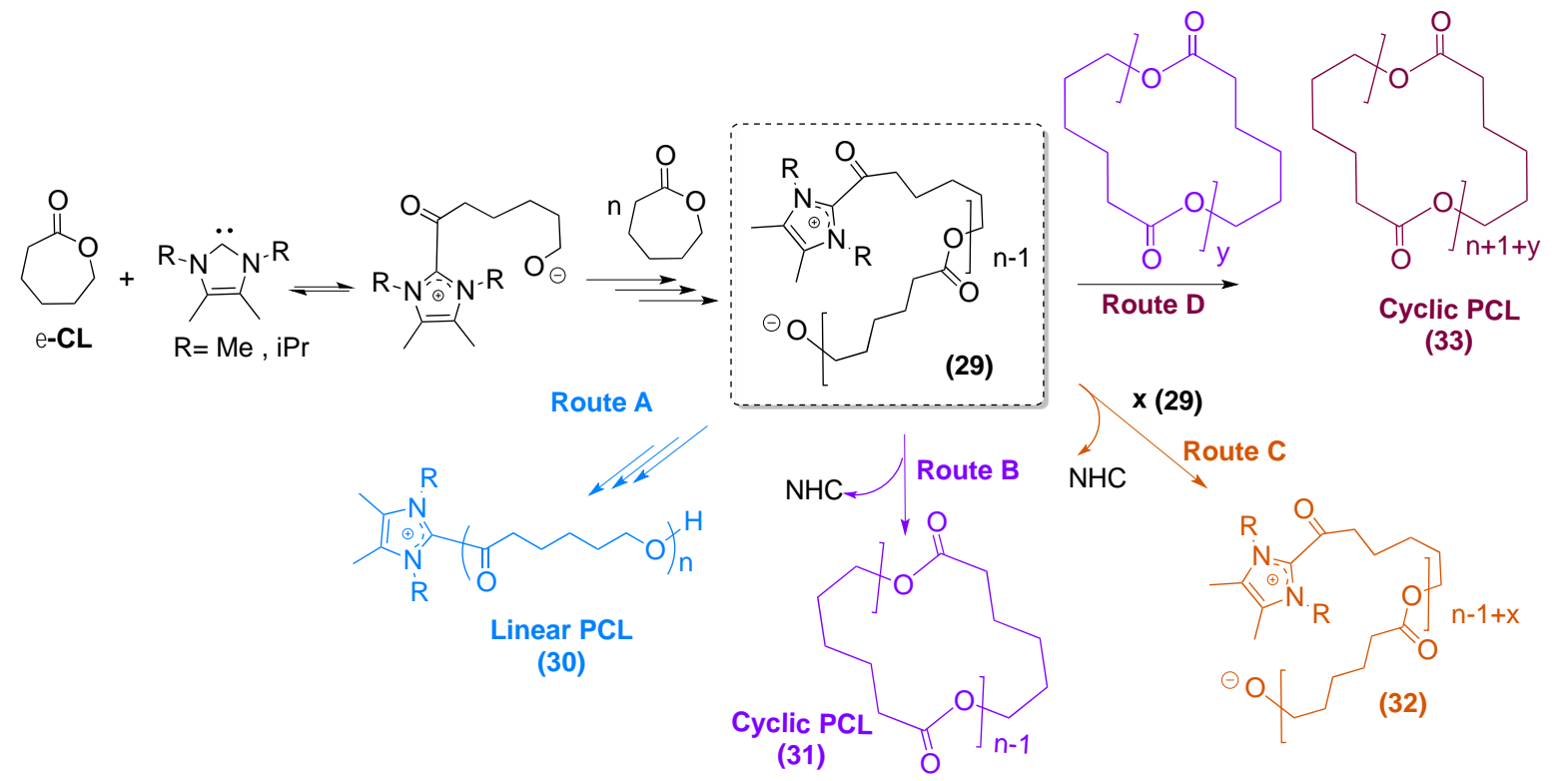

Scheme 65. Proposed mechanism for ZROP of $\varepsilon$-CL using NHCs and related side reactions. [188, 189]

The direct ROP of other monomers, such as cyclic siloxanes, cyclic phosphates and $\mathrm{N}$ carboxyanhydrides initiated by NHCs was also reported. For instance, Waymouth et al. described the NHC-initiated ZROP of 2,2,5,5- tetramethyl-2,5-disila-1-oxacyclopentane (TMOSC, see Figure 3) ) using a series of NHCs in THF at r.t.[190] 1,3,4,5-Tetramethylimidazol-2-ylidene (NHC18) and 1,3-bis-isopropyl-4,5-dimethylimidazol-2-ylidene (NHC19) proved the most efficient initiators (> 95\% of monomer conversion reached after $5 \mathrm{sec}$ ), yielding cyclic PTMOSC's, i.e. poly(carbosiloxane)s, of very high molar masses, $M_{n}=587,000 \mathrm{~g} \cdot \mathrm{mol}^{-1}$ $(\Theta=1.74)$ and $942,000 \mathrm{~g} \cdot \mathrm{mol}^{-1}(\Theta=3.18)$, respectively. The more sterically hindered 1,3bis(2,6-diisopropylphenyl)imidazol-2-ylidene (NHC8) did not exhibit any activity for this polymerization, presumably due to its bulkiness. Under the same conditions, both NHC18 and NHC19 were also found to mediate the ZROP of 2-isopropoxy-2-oxo-1,3,2-dioxaphospholane (iPP, see Figure 3). The polymerization rapidly occurs to generate cyclic poly(alkylene phosphates), namely poly(iPP), of molecular weights ranging from 55,000 to $202,000 \mathrm{~g} \cdot \mathrm{mol}^{-1}$ $(\Xi<1.3)$. [191] new ref Waymouth Macromol 2014. In the same manner, the ZROP of $N$ carboxyanhydrides initiated with various imidazol-2-ylidenes (NHCs 2-3; 7-8), allowed synthesizing cyclic polypeptoids in a controlled manner $\left(M_{n}=3,000-30,000 \mathrm{~g} \cdot \mathrm{mol}^{-1}\right.$ and $\bigoplus=1.2) \cdot[192]$

The NHC-initiated zwitterionic polymerization of (meth)acrylic monomers, through repeated and selective 1,4-conjugate addition reactions, was also described. In 2012, Chen et 
al. reported the direct polymerization of both MMA and a biomass-derived cyclic analog, $\gamma$ methyl- $\alpha$-methylene- $\gamma$-butyrolactone (MMBL). Three different NHCs, namely, $\mathrm{NHC}^{\mathrm{tBu}}(=\mathrm{NHC})$, $\mathrm{NHC}^{\mathrm{Mes}}(=\mathrm{NHC7})$ and 1,3,4-triphenyl-4,5-dihydro-1H-1,2,4-triazol-5-ylidene (TPT = NHC26), were tested as chain starters, in DMF at r.t.[17, 19] $\mathrm{NHC}^{\mathrm{tBu}}$ proved the most active $\left(\mathrm{NHC}^{\mathrm{tBu}}>\right.$ $\mathrm{NHC}^{\mathrm{Mes}}>\mathrm{TPT}$ ), converting up to 10,000 eq. of MMBL in 5 min only, and leading to PMMBL of $M_{n}=70,000-85,000 \mathrm{~g} \cdot \mathrm{mol}^{-1} \quad(\Theta=1.36-2.11)$, regardless of the $[\mathrm{MMBL}]_{0} /[\mathrm{NHC}]_{0}$ ratio employed. In contrast to MMBL, the polymerization of MMA could only be induced by $\mathrm{NHC}^{\mathrm{tB} u}$, leading to PMMA of $M_{n}=33,300 \mathrm{~g} \cdot \mathrm{mol}^{-1}(\Theta=1.99)$. The polymerization was proposed to proceed through a 1,4-conjugate addition reaction of the NHC onto MMA, generating an imidazolium enolate 34 (Scheme 66), that can propagate the polymerization.[17, 19, 193]

In sharp contrast, under the same conditions (in DMF, r.t), Taton et al. found that the reaction of 1,3-bis-(diiso-propyl)imidazol-2-ylidene $\left(\mathrm{NHC}^{\mathrm{iPr}}=\mathrm{NHC2}\right)$ with $\mathrm{MMA}$ afforded an unprecedented imidazolium enolate cyclodimer ( $\mathrm{NHC}^{\text {iPr: }}$ MMA $\left.=1: 2\right),[193]$ evidencing the remarkable selectivity of NHCs towards MMA.

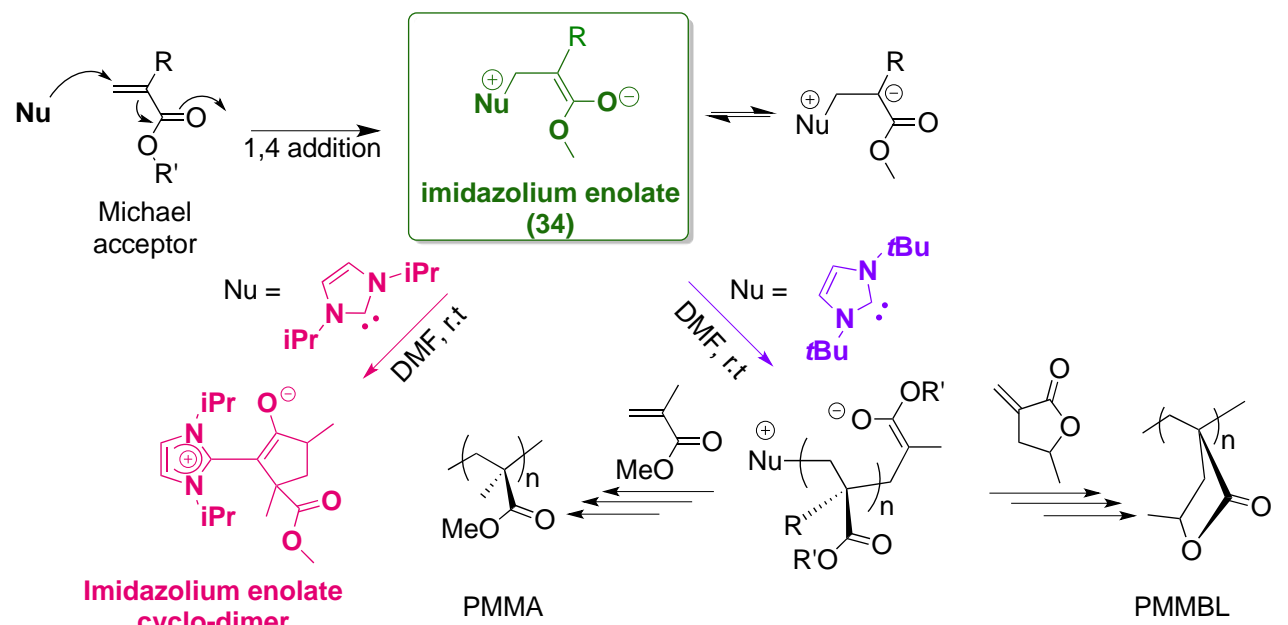

Scheme 66. NHC reactions towards MMA.[193]

In a recent addition, TPT was found to efficiently catalyze the conversion of dimethacrylates into unsaturated polyesters of $M_{w}$ up to $28,400 \mathrm{~g} \cdot \mathrm{mol}^{-1}$ and $Ð=1.1-1.9$, in toluene at $80{ }^{\circ} \mathrm{C}$ (Scheme 67).[194] This reaction was thought to involve the initial conjugate addition of TPT onto the monomer, forming the triazolium enolate 35 (Scheme 67). Upon proton transfer, the resulting nucleophilic diaminoalkene 36 could react in a 1,4-fashion with another monomer molecule to yield the new triazolium enolate 37 . This new step-growth polymerization, referred to as proton $(\mathrm{H})$ transfer polymerization $(\mathrm{HTP})$, was proposed to proceed by repeated conjugate addition / proton transfer / conjugate addition / proton transfer/ NHC release sequences.[194] 


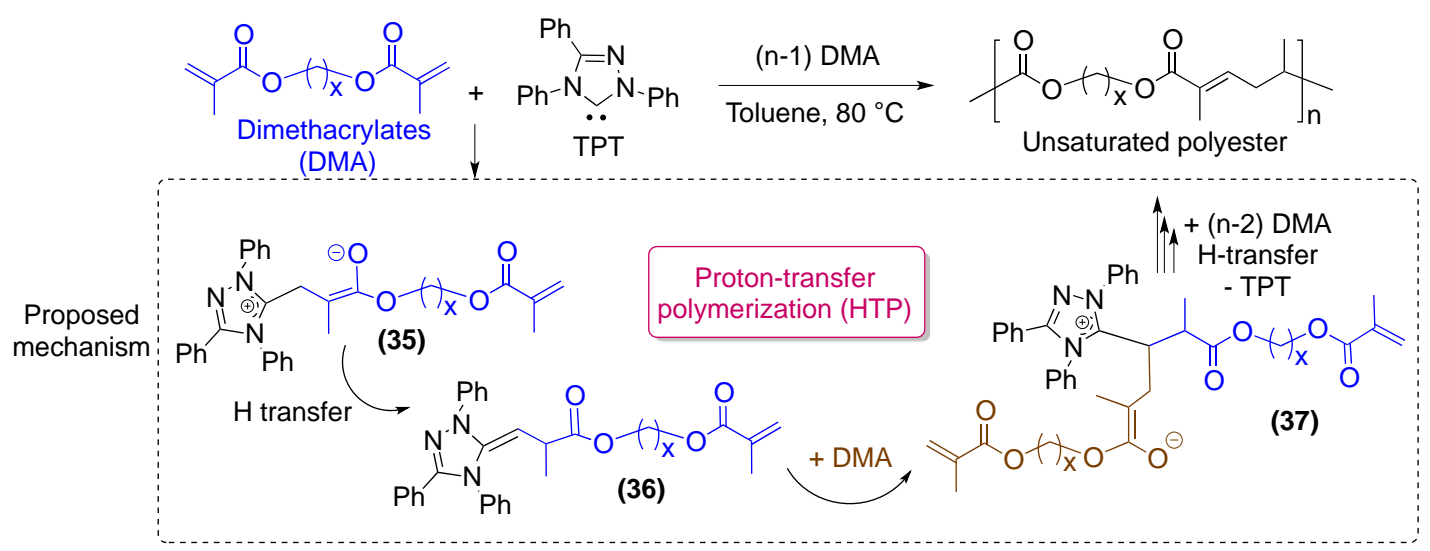

Scheme 67. Proposed mechanistic scenario for the HTP of dimethacrylates forming unsaturated polyesters. [194]

Interestingly, the same TPT carbene was employed by Matsuoka et al. to catalyze the oxa-Michael addition polymerization of hydroxy-functionalized acrylate monomers, in bulk at r.t.[195]. Such a polymerization produced saturated poly(ester-ether)s of $M_{n}=750-2,400 \mathrm{~g} \cdot \mathrm{mol}^{-1}$ ( $\left.\theta=1.2-3.8\right)$ via a step-growth process, instead of poly(acrylate)s (as in the case of $\mathrm{NHC}^{\mathrm{tBu}}$ ). Based on NMR and mass spectrometry analyses, the authors proposed an AMM, where TPT activates the monomer by conjugate addition. The ensuing triazolium enolate 38a undergoes a proton transfer, generating a hydroxyl functional triazolium 38b and an alkoxide growing chain end (39) that can add onto the monomer via an oxa-Michael type reaction. Upon proton transfer, a new alkoxide growing chain end is created, leading to the propagation by iterative oxa-Michael addition.

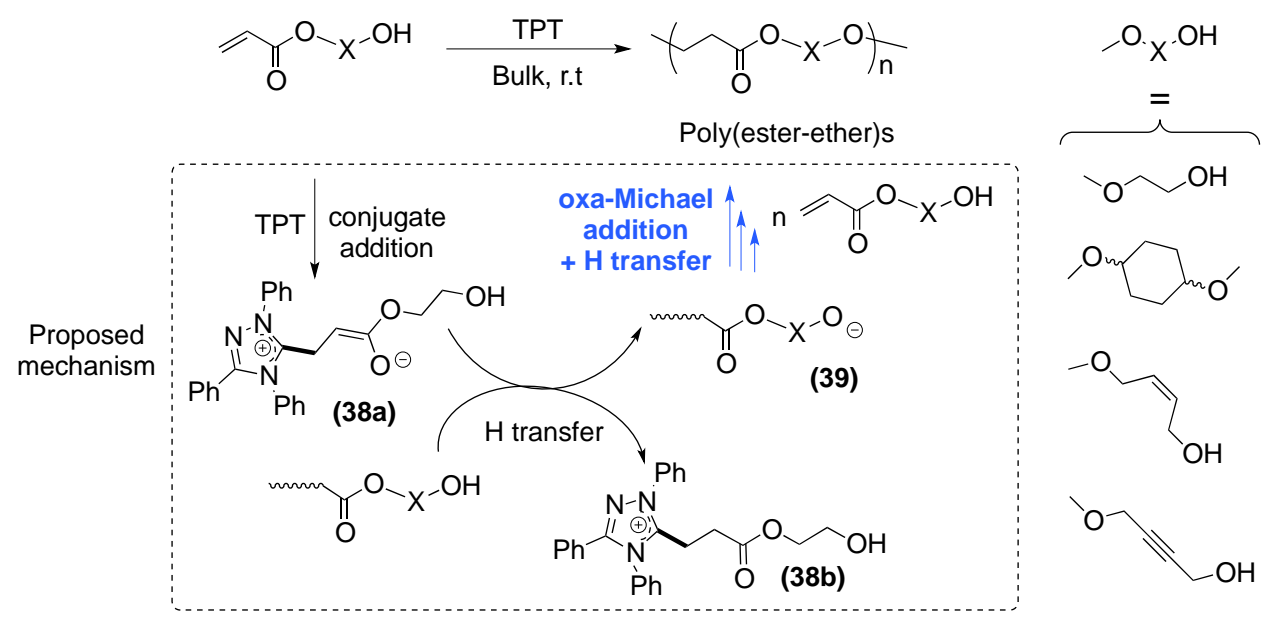

Scheme 68. Proposed mechanism of the oxa-Michael addition polymerization of acrylates catalyzed by TPT.[195]

Besides free NHCs, latent initiating systems were investigated by Buchmeiser et al. for polymerization reactions. For instance, $\mathrm{NHC}-\mathrm{CO}_{2}$ adducts, including imidazolium-, imidazolinium-, tetrahydropyrimidinium- and diazepinium-based compounds, were shown to behave as thermally latent chain starters for the ZP of MMA, in bulk or in solution (DMSO or DMF) at $80^{\circ} \mathrm{C} .[18]$ 
Deactivation of the $\mathrm{NHC}$ by intra-molecular $\mathrm{C}-\mathrm{H}$ insertion of the carbene center into a $\mathrm{CH}_{3}{ }^{\text {Mes }}$ was proposed to account for the inactivity of the diazepinium-based carbene (Scheme 69). $[18,196]$

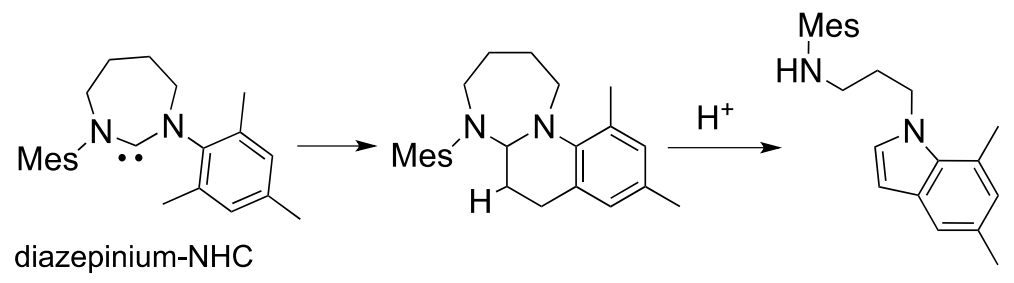

Scheme 69. Intra-molecular C-H bond insertion of diazepinium-type NHCs.[18, 196] Scheme modified

In contrast, $\mathrm{NHC}^{\mathrm{tBu}}-\mathrm{CO}_{2}$ enabled the direct polymerization of MMA in solution and in bulk at $85^{\circ} \mathrm{C}$.[18] With this NHC-precursor, PMMA's with molar masses $M_{n}$ of 25,000 g.mol-1 could be obtained, with a rather broad molar mass distribution $(\Theta=2.6-2.7)$, these values $\left(M_{n}\right.$ and $\left.\boxminus\right)$ being comparable to those reported by Chen et al.[17] for the polymerization of MMA in DMF at r.t. with the bare $\mathrm{NHC}^{\mathrm{BBu}}$.

Tetrahydropyrimidinium-2-carboxylates were also suitable initiators for the polymerization of MMA in DMSO $\left(\mathrm{M}_{\mathrm{n}}=80,000 \mathrm{~g} \cdot \mathrm{mol}^{-1}\right.$ and $\left.\Xi=1.82\right)$.[18] In this case, however, the NHC-CO adducts likely operated as latent Brønsted bases rather than nucleophiles. Indeed, it was proposed that these highly basic NHCs could deprotonate DMSO, generating the so-called "dimsyl" anion (40) that could further initiate the anionic polymerization of MMA (Scheme 70) with the tetrahydropyrimidinium as bulky counteraction (not a ZP in this case). Alternatively, a GTP like mechanism could also be operative, where the initiator would be a NHC-enolate adduct (instead of an ion pair). In this case, the NHC would be transferred to the growing enolate chain end, based on the higher electrophilicity of these saturated NHCs.

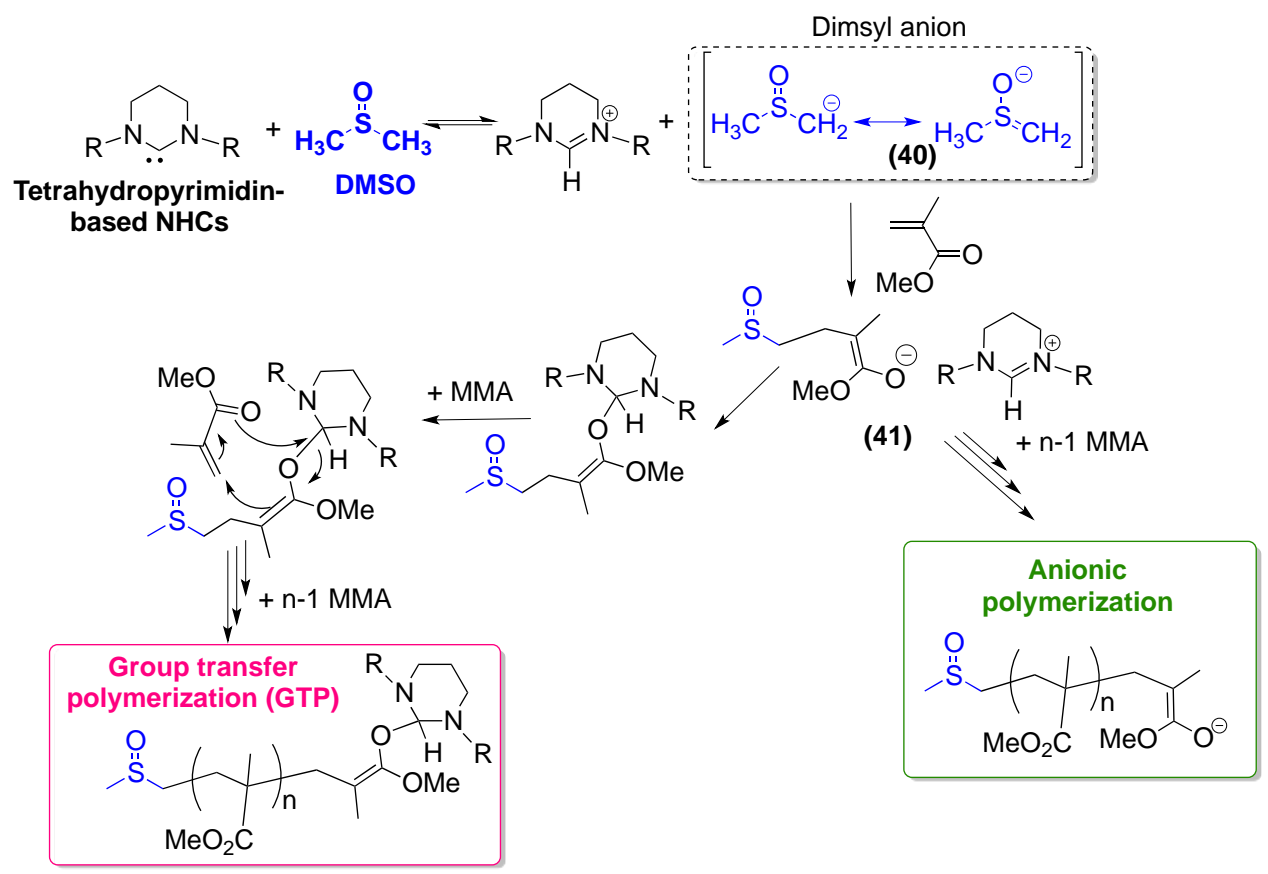


Scheme 70. Reaction of DMSO with NHCs and possible anionic polymerization.[18]

The same group also described the direct ROP of $\varepsilon$-caprolactam $(\varepsilon-C L A)$ initiated by various $\mathrm{NHC}-\mathrm{CO}_{2}$ adducts (NHCs 22-25; $34-38$ in Figure 2).[197] Polymerizations were conducted under rather harsh conditions, i.e. in bulk at $180^{\circ} \mathrm{C}$, allowing the preparation of polyamide-6 (PA-6) (Scheme 71).[197]. Importantly, this polymerization also evidenced that $\mathrm{NHC}-\mathrm{CO}_{2}$ adducts and related free NHCs thus delivered could withstand such a high temperature. Tetrahydropyrimidinium-based carbenes were more active than imidazol(in)ium compounds, giving after 45 min PA- 6 of molar masses $M_{\eta}=140,000-420,000 \mathrm{~g} \cdot \mathrm{mol}^{-1}$ (as determined by viscometric measurements). The broadened dispersity observed and the high molar masses obtained suggested incomplete initiation or other side reactions, such as chain transfer, as expected for the anionic-like ROP of $\varepsilon$-CLA.[198, 199] Nevertheless, the inhomogeneity of the system caused by the precipitation of PA-6 from the monomeric melt, could also explain these results.

The activity of tetrahydropyrimidinium carboxylates was related to the basicity of corresponding free carbenes, suggesting a basic mechanism where the monomer was anionically polymerized (see insert in Scheme 71). Thus, polymerization started by deprotonation of the lactam by the $\mathrm{NHC}$, forming the activated monomer amide anion $\mathbf{4 2}$ that could initiate the ROP (see insert in Scheme 71).

$\mathrm{NHC}-\mathrm{CO}_{2}$ adducts were also effective pre-catalysts for the bulk homopolymerization of laurolactam (LL), leading to PA-12 of molar masses $M_{n}$ of $14,000 \mathrm{~g} \cdot \mathrm{mol}^{-1}(\Theta=2.2-3.1) \cdot$ [200] The copolymerization of LL and $\varepsilon$-CLA gave a gradient copolymer, $\varepsilon$-CLA being incorporated preferentially at the onset of the polymerization.
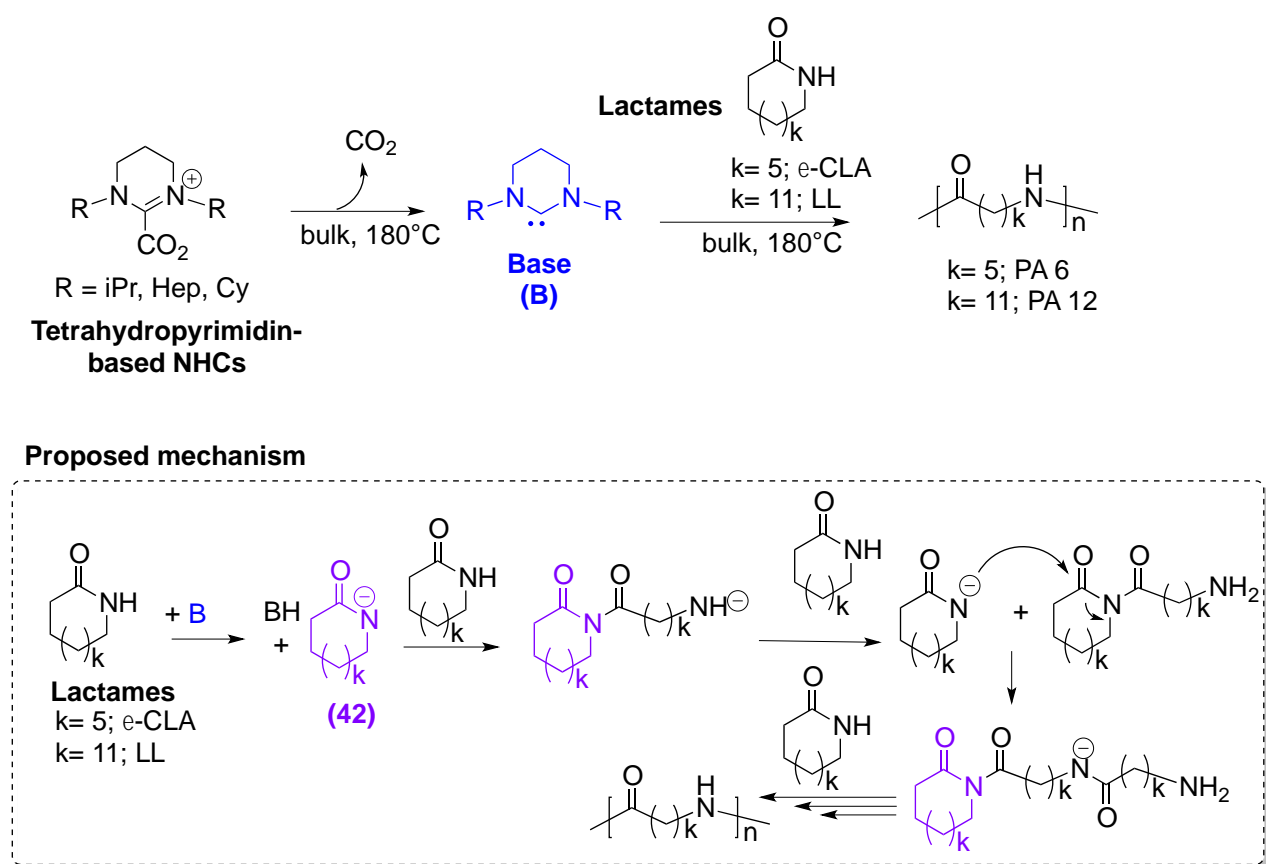

Scheme 71. Anionic ROP of lactams ( $\varepsilon-C L A$ and LL) using NHCcarboxylates.[197, 200] 
Imidazolium carboxylates, including $\mathrm{NHC}^{\text {Mes- }} \mathrm{CO}_{2}$, 1,3-bis(2,6-diisopropylphenyl) imidazolium-2-carboxylate $\left(\mathrm{NHC}^{\text {iPrPh}}-\mathrm{CO}_{2}, \quad \mathrm{NHC8}\right.$ in Figure 2) and 1,3-bis(2,6bis(diphenylmethyl)-4-methylphenyl) imidazolium-2-carboxylate $\left(\mathrm{NHC}^{\mathrm{PrPh}}-\mathrm{CO}_{2} ; \mathrm{NHC9}\right)$, were applied to the ROP of $\beta-\mathrm{BL}$ in THF or/and toluene at $60^{\circ} \mathrm{C}$.[201] The most bulky $\mathrm{NHC}^{\mathrm{iPrPh}}-\mathrm{CO}_{2}$ and $\mathrm{NHC}^{\text {iPrPh*}}-\mathrm{CO}_{2}$ proved less reactive than $\mathrm{NHC}^{\text {Mes }}-\mathrm{CO}_{2}$, leading to a lesser control over molar masses. The polymerization induced by $\mathrm{NHC}^{\text {Mes }}-\mathrm{CO}_{2}$ proceeded in $10 \mathrm{~min}(80 \%)$, yielding a PBL of $9,200 \mathrm{~g} \cdot \mathrm{mol}^{-1}(\Theta=1.46)$. Molar masses increased with the initial $[\beta-\mathrm{BL}]_{0} /[\mathrm{NHC}]_{0}$ ratio, and led to PBL's of $M_{n}=6,300-37,800 \mathrm{~g} \cdot \mathrm{mol}^{-1}(\Theta=1.12-1.47$ ), suggesting a controlled polymerization. $D, \mathrm{~L}-\mathrm{LA}$ was also polymerized under the same conditions, PLA's with $\mathrm{M}_{\mathrm{n}}=6,300-13,400 \mathrm{~g} \cdot \mathrm{mol}^{-}$ ${ }^{1}(\Xi=1.22-1.83)$ being quantitatively prepared after $30 \mathrm{~min}$. Statistical PBL-co-PLA copolymers could also be prepared by simultaneous copolymerization, while sequential copolymerization ( $\beta$-BL first polymerized) only led to PBL-based homopolymers.[201]

Protected NHCs were also applied to the thermal curing of bisphenol A diglycidyl ether (BADGE), using phthalic acid anhydride (PhA) or hexahydrophthalic anhydride (HHPA) as hardener, as reported by Buchmeiser et al. for the fabrication of fiber-based composite materials. [202, 203] new ref Buchmeiser Macromol 2014 + Macromol. Mater. Eng. 2015. All NHCs precatalysts, i.e imidazoli(ni)um- and tetrahydropyrimidinium-based NHCs, were able to bring about a fast and full curing at $140-160^{\circ} \mathrm{C}$. Using $0.1-1 \mathrm{~mol} \%$ of NHC precursor, the asprepared thermosets exhibited high glass transition temperatures $\left(127-144^{\circ} \mathrm{C}\right)$, similar to those of conventionally derived materials. From a mechanism point of view, the authors proposed a NHC-triggered ring-opening of cyclic anhydrides (Scheme 72b), rather than the generally admitted ring-opening of oxirane moieties by conventional imidazole-based accelerators (Scheme 72a).

a)

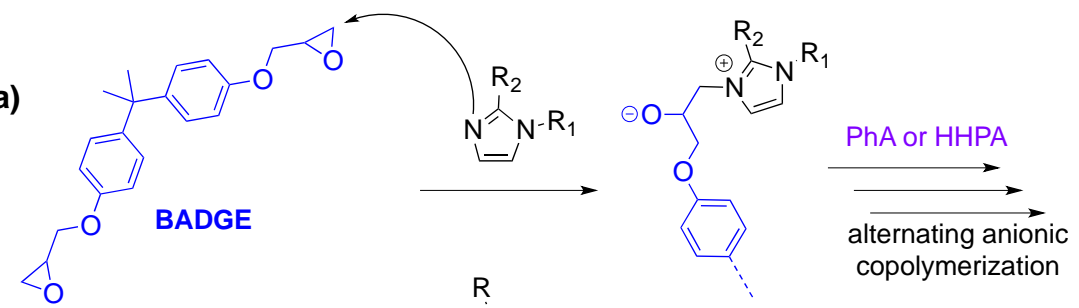

b)

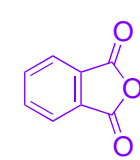

PhA

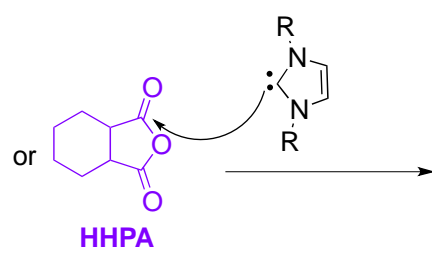

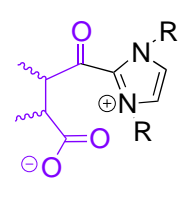

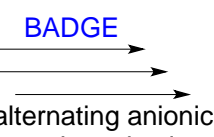

copolymerizo

Scheme 72. Proposed mechanism for curing of epoxide/anhydride resins using imidazole-based accelerators (a) and NHCs (b). [202, 203] new scheme added

By analogy with the NHC-mediated ROP of D,L-LA, Waymouth et al. recently employed DBU and DBN amidines as direct initiators, in absence of any alcohol initiator.[204] Cyclic PLA's 
with $\mathrm{M}_{\mathrm{n}}$ ranging from 32,000 to $53,000 \mathrm{~g} \cdot \mathrm{mol}^{-1}(\Theta \leq 1.6)$ were obtained in dichloromethane or THF/ dichloromethane, but no clear relationship was found between these molar masses and the initial ratio $[\mathrm{LA}]_{0} /[\mathrm{DBX}]_{0}(\mathrm{DBX}=\mathrm{DBU}$ or $\mathrm{DBN})$. A similar behavior was observed for the ZROP of lactide by NHCs, which was attributed to a slow and reversible initiation step.[12, 205] Theoretical studies supported the occurrence of a zwitterionic mechanism through the formation of amidinium alkoxide zwitterions, generating a mixture of linear and cyclic PLA's, as observed for the ZROP with NHCs (see Scheme 65).[12, 205]. Compared to DBU initiator, isothioureas (see Scheme 64 for related structure) were demonstrated to be more selective for producing cyclic PLA's $\left(M_{n}=48,000-66,000\right.$ g.mol ${ }^{-1}(\Theta \leq 1.6)$ without appreciable linear contaminants.[206]

Poly(3-hydroxybutyrate)s (PHB's), with $\alpha, \omega$-DBU- $N$-acylcrotonate chain-ends and molar masses $M_{n}=2,600-9,400$ g.mol${ }^{-1}(\Theta=1.16-1.52)$, were achieved by Guillaume, Carpentier et al., when performing the ROP of $\beta-B L$ in bulk at $60^{\circ} \mathrm{C}$ with DBU (Scheme 73).[207] While similar polymers were obtained when using the guanidine TBD, higher molar masses with a narrow dispersity were obtained with the BEMP phosphazene $\left(M_{n}=4,200-24,600 \mathrm{~g} \cdot \mathrm{mol}^{-1} ; \Xi=1.07\right.$ 1.22).[207]

$\mathrm{DBU}, \mathrm{TBD}$ and BEMP proved also efficient to trigger the ROP of benzyl $\beta$-malolactone ( $\beta$ $\mathrm{MLABe}$ ), leading to poly(3-benzyloxybutyrate)s (PMLABe's) of molar masses $M_{\mathrm{n}}=$ $18,500 \mathrm{~g}^{\mathrm{mol}}{ }^{-1}$ and $€=1.20-1.65$ (Scheme 73).[208] Both DBU and BEMP appeared more powerful than TBD for the ROP of MLABe. Although the mechanism of the ROP of $\beta$-BL and MLABe mediated by BEMP, TBD or DBU was not fully established, formation of zwitterionic species $\mathbf{A}_{\mathbf{i}}$ (initiator-BL 1:1 adduct) via an AMM (see insert in Scheme 73), was postulated based on chain-ends identified by NMR and by MALDI-ToF. ${ }^{1} \mathrm{H}$ NMR analyses of $\beta$-BL/BEMP mixture indeed revealed the presence of crotonate groups from the early stage of the reaction, and their relative intensity as compared to BEMP signals remained constant during the ROP; hence an anionic ROP mechanism could be ruled out.

The sequential or simultaneous copolymerization of $\beta-B L$ and racemic $\beta-M L A B e$ was also investigated by the same group.[209] Remarkably, only BEMP afforded PMLABe- $b$-PBL block copolymers following either a simultaneous $\left(\mathrm{M}_{\mathrm{n}}=17,700 \mathrm{~g} \cdot \mathrm{mol}^{-1} ; \boxminus=1.44\right)$ or a sequential approach $\left(\mathrm{M}_{\mathrm{n}}=30,000 \mathrm{~g} \cdot \mathrm{mol}^{-1} ; \ominus=1.46\right)$, regardless of the order of the co-monomer addition. In all cases, formation of corresponding $\omega$ - $N$-acylcrotonate functionalized PMLABe-PBL copolymers were evidenced. With both DBU and TBD, only the sequential addition pathway provided block copolymers $\left(M_{n}=7,200-55,200 \mathrm{~g} \cdot \mathrm{mol}^{-1} ; \Xi=1.25-1.42\right)$, homopolymers being obtained upon performing simultaneous copolymerization. 


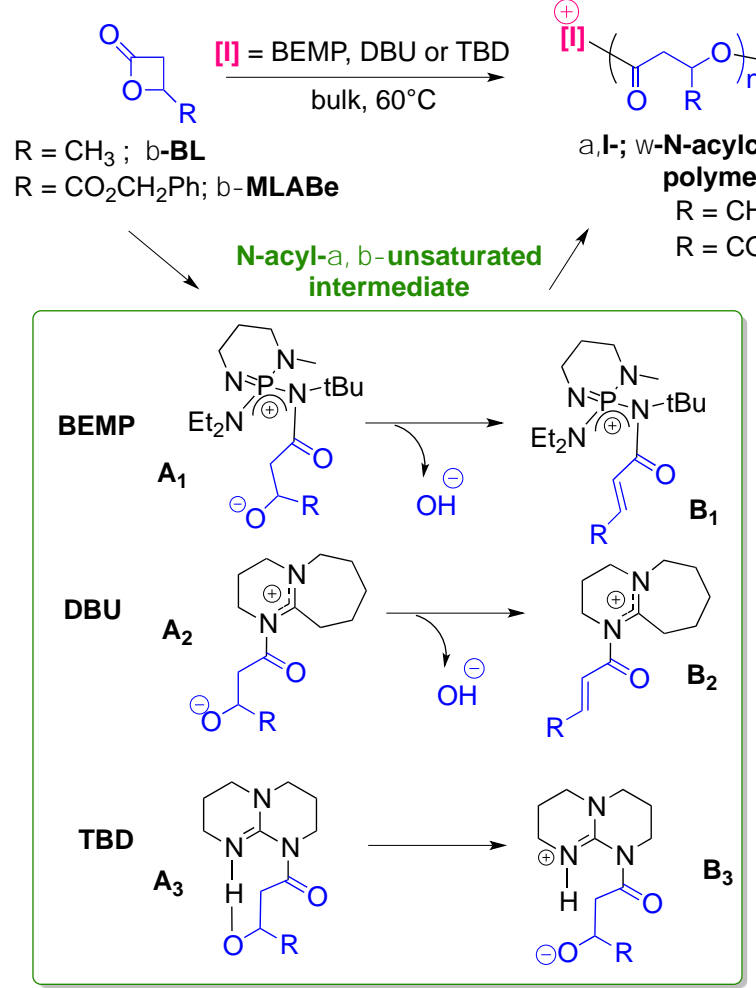

Scheme 73. Direct ROP of $\beta$-BL and benzyl $\beta$-malolactone (MLABe) using BEMP phosphazene, DBU (amidine) and TBD (guanidine). [207-209]

The DBU-initiated ROP of a glucose-based cyclic carbonate, namely, methyl-4,6-Obenzylidene-2,3-O-carbonyl-a-D-glucopyranoside (MBCG), was also reported by Endo et al. (Scheme 74).[210, 211] new ref Endo Macromol 2005+JPSA 2013. Using the initial ratio $[\mathrm{MBCG}]_{0} /[\mathrm{DBU}]_{0}=25$, the polymerization produced the corresponding poly(MBCG)s in $71-98 \%$ yield with $M_{n}=3,600-11,000 \mathrm{~g} \cdot \mathrm{mol}^{-1}(\Xi=1.24-1.49$ ), at r.t in THF or DMF. The polymerization was faster in DMF than in THF, presumably due to the better solvation and least aggregation of the zwitterionic intermediate in this more polar and dissociative solvent. The lower molar masses observed in DMF were attributed to the occurrence of chain transfer reactions such as backbiting.

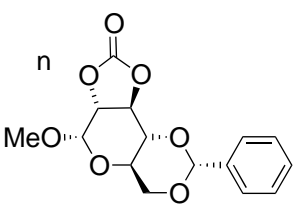

MBCG

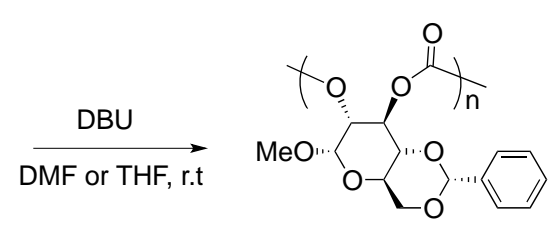

poly(MBCG)
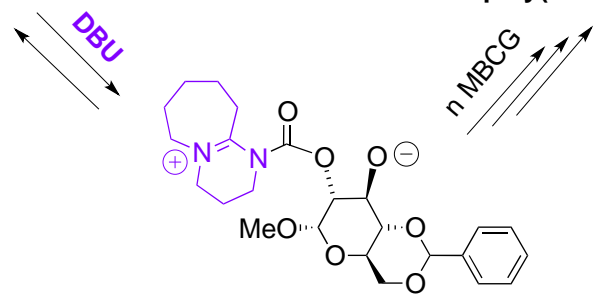

zwitterion intermediate 
Scheme 74. DBU-initiated ROP of glucose-based carbonate monomer MBCG.[210, 211] new scheme added

Chen et al. reported that the $t$-BuP 4 phosphazene could directly initiate the polymerization of both $\alpha$-methylene- $\gamma$-butyrolactone (MBL) and its $\gamma$-methylated analogue (MMBL) in DMF at r.t. (Scheme 75).[212] Using 0.02 mol\% of $t-\mathrm{BuP}_{4}, \mathrm{MMBL}$ polymerizations went to completion within only $1 \mathrm{~min}$ of reaction, forming high molar masses PMMBL's $\left(M_{n}=23,200-111,000 \mathrm{~g} \cdot \mathrm{mol}^{-1}\right)$ but of rather high dispersity $(\Theta=1.89-3.98)$. The polymerization of the parent monomer, MBL, proved significantly slower and was incomplete ( $79 \%$ conversion after 7 days; $M_{n}=1,600-104,000 \mathrm{~g} \mathrm{~mol}^{-1}$ and $\Xi=1.50$ ). The drastic effect of $\gamma$-methyl substitution on the MBL ring (in MMBL) on the polymerization activity might be ascribed to the enhanced nucleophilicity of the active species, ultimately leading to complete monomer conversion with no side reactions.

The authors proposed that the polymerization proceeded through deprotonation of MMBL by $t$-Bu- $\mathrm{P}_{4}$, generating a highly reactive anionic allylic species $\mathbf{4 3}$ stabilized by the bulky countercation $\left[t-\mathrm{BuP}_{4}-\mathrm{H}\right]^{+}$. Hence, propagation of polymethacrylate chains would occur through a truly anionic (i.e. non zwitterionic) mechanism (Scheme 75). Note that two isomeric chain ends [l] (see insert in Scheme 75) could be formed, depending on the nature of the carbanionic initiating species (positions 1 or 3 of the allylic system).

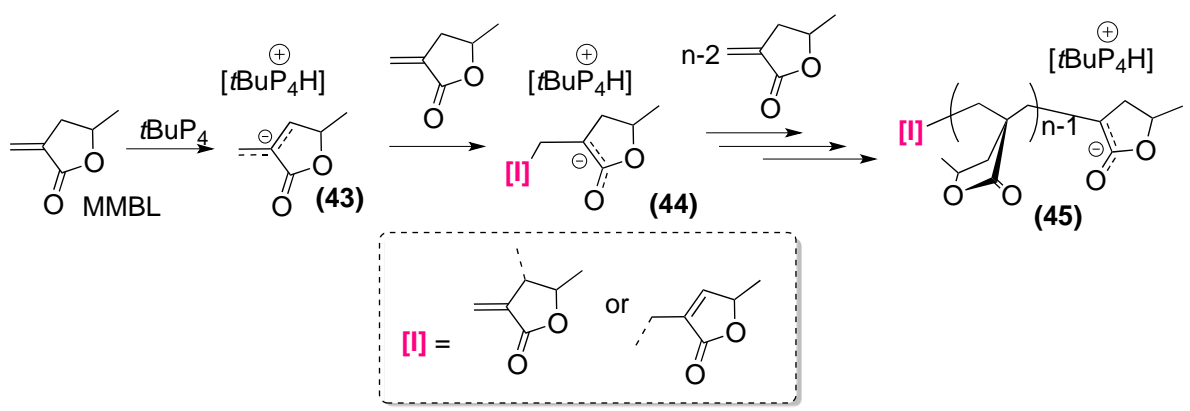

Scheme 75. Polymerization of MMBL direct initiated by $t \mathrm{BuP}_{4}$ as initiator.[212]

(+)Sparteine was also employed by Coulembier et al. as direct initiator for the ROP of L-LA in dichloromethane at $21^{\circ} \mathrm{C}$ (Scheme 76).[213] Even though the polymerization was much slower than when initiated by an alcohol (and a catalytic amount of sparteine), cyclic PLLA's of $M_{n}=8,000-20,000 \mathrm{~g} \cdot \mathrm{mol}^{-1}(\Theta=1.4-1.7)$ could be achieved. (+)Sparteine would play the role of a di-nucleophile, forming the zwitterionic species 46 (Scheme 76), and the bis(zwitterion) $\mathbf{4 7}$, after the addition of one and two monomer units, respectively. Subsequent addition of a few LLA molecules might generate the bis(zwitterion) 48, which would undergo a double "tail-biting" type reaction, yielding cyclic PLA's while regenerating sparteine. 


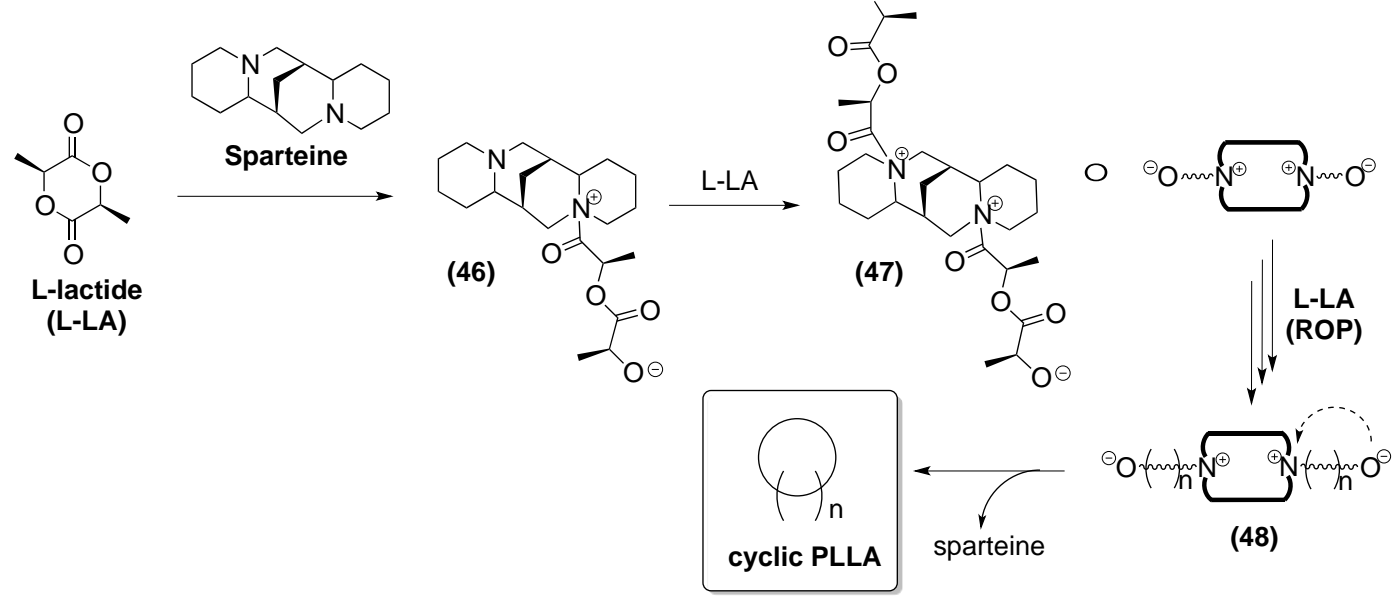

Scheme 76. Sparteine-mediated ROP of L-lactide (L-LA).[213]

Besides Brønsted and Lewis bases, a few organic acids were also employed as direct initiators for ROP. For instance, a variety of arylhydrogen-phosphates and aryldihydrogenphosphates proved efficient activators for the ROP of $\varepsilon$-CL and $\delta$-VL in bulk at $80^{\circ} \mathrm{C}$ (Scheme 77).[214] PCL's of molar masses $M_{n}=31,300-90,700 \mathrm{~g} \cdot \mathrm{mol}^{-1}$ and PVL's of $M_{n}=28,900-90,400$ g.mol ${ }^{-1}$ with $\Theta=1.05-1.35$ were obtained in this way. A cationic-AMM, involving initial protonation of the monomer by the acid, was proposed (see insert in Scheme 77). The phosphonate anion being nucleophilic enough to add to the activated monomer, it eventually triggered ring-opening, giving the hydroxyl intermediate 49 , capable of initiating the next ring-opening of another activated monomer.

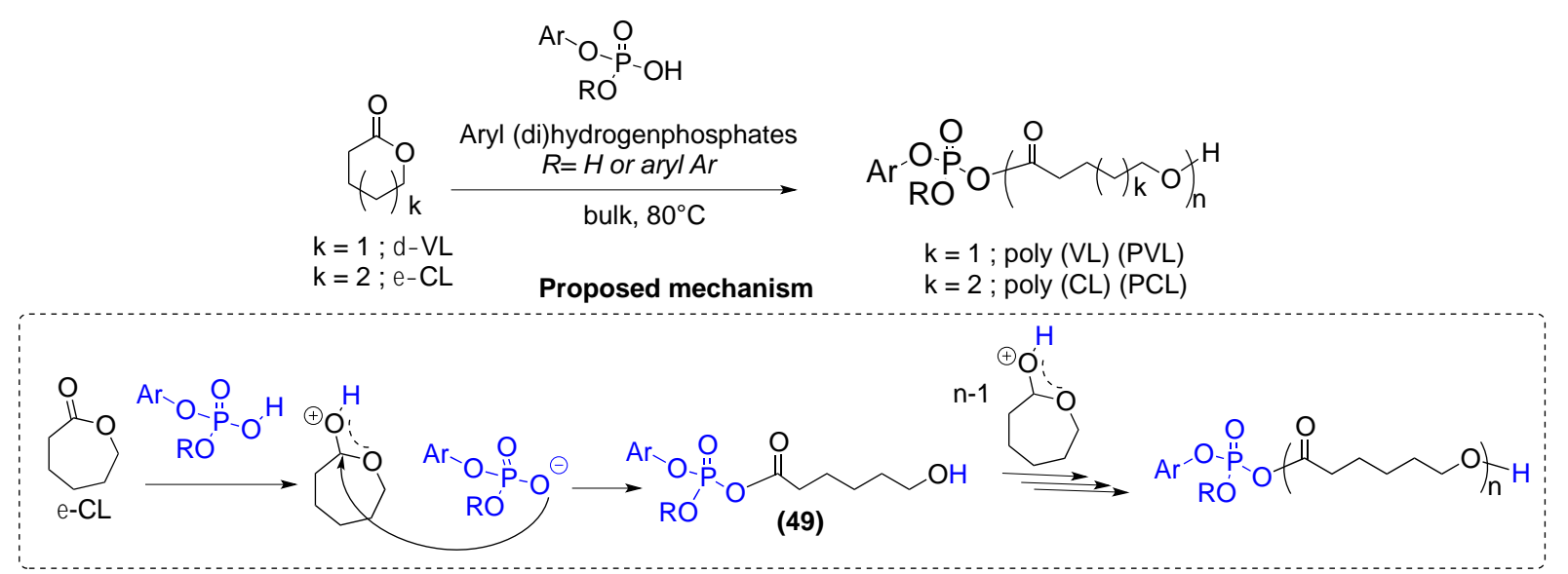

Scheme 77. ROP of $\delta$-VL and $\varepsilon-C L$ using aryl (di)hydrogenphosphates as initiators. [214]

Recently, Chen et al. reported the use of Lewis pairs (LP's) based on monocomponent phosphine-borane systems for the polymerization of the aforementioned biosourced $\gamma$-MMBL (Scheme 78).[23] The polymerization was conducted in dichloromethane at r.t. and took place by a cooperative activation of the monomer via both a Lewis acidic and a Lewis basic activation. For an initial ratio $[\gamma-\mathrm{MMBL}]_{0} /[\mathrm{LP}]_{0}=800$, quantitative conversion was reached in $2 \mathrm{~min}$, producing high molar masses PMMBL $\left(M_{n}=104,000 \mathrm{~g} \cdot \mathrm{mol}^{-1} ; \quad D=1.34\right)$. Since boron is 
considered as a "semi-metal", this Lewis pair polymerization can be considered as a "semi" metal-free polymerization.

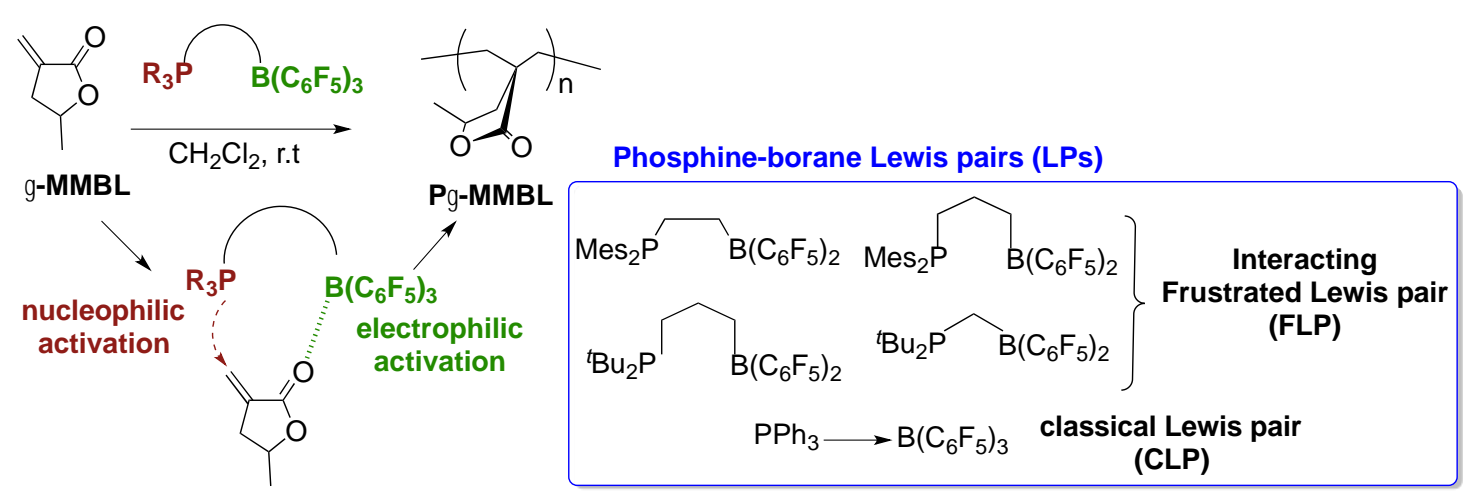

Scheme 78. Phosphine-Borane Lewis pairs (LPs) induced polymerization of $\gamma$-MMBL.[23]

In summary, small organic molecules were recently employed as direct initiators, i.e. in absence of any other co-activator, to trigger not only the (Z)ROP of cyclic monomers (lactide, lactones and lactams), but also the zwitterionic vinyl polymerization of (meth)acrylic monomers. For these organo-initiated polymerizations, metal-free activators included Lewis bases such as NHCs, but also amidines or phosphazenes. Only one example of an acid initiator (i.e. (di)hydrogenphosphate) was reported. However, these direct polymerizations were usually not very well-controlled, giving rather high dispersities. In addition, the reactant structures (initiator and monomer) drastically influenced the topology of the final polymer, with either cyclic or/and linear structures being generated.

\section{Concluding remarks and future challenges}

This review has encompassed the contributions over the past 4 years regarding organopolymerization reactions. The diverse reactivity and selectivity of small organic molecules have been efficiently transferred in recent years, from organocatalysis in organic synthesis to precision macromolecular chemistry. Organic catalysts are generally easily available and can improve polymerization kinetics while providing a good control over the polymerization process by favouring selective propagation steps over side reactions. In some cases, they exhibit performances that compare or even outperform those of commonly used organometallic catalysts, making them powerful alternatives for the preparation of polymers free of any metallic residues.

Some of metal-free polymeric materials have demonstrated valuable physico-chemical properties as well as in vivo biocompatibility, making them promising materials for applications in tissue engineering, drug delivery and regenerative medicine. For instance, cationic polycarbonates were found to form dynamic micelles that allowed bacterial cell interactions to be increased and proved strong antimicrobial films against drug-resistant bacteria.[215-217] 
Copolymers such as PEO-polycarbonates or PEO-polyphosphoesters were demonstrated to self-assemble into micelles with high loading capacity of the hydrophobic anticancer drug paclitaxel (PTX). These PTX-loaded micelles were preferentially accumulated in tumor tissue with limited distribution to healthy organs.[218-220] These few examples of metal-free synthesized polymers in bio-applications illustrate the expansion of organopolymerization, from a precise chemistry to specific applications.

The absence of metal traces is not only sought after in biomedicine, but also in applications such as packaging and microelectronics, where metallic residues could interfere with the polymer end use. Moreover, it should be kept in mind that these residues could drastically shorten the polymer lifespan due to an increase in degradation during either polymer processing or weathering.[221] Although toxicity issues are often neglected concerning organic activators, health and environmental risks of these compounds in the final material are so far almost unknown and need to be clarified. For instance, in a very recent contribution, Mespouille, Coulembier, Dubois et al. demonstrated that functionalized thioureas showed an important cytotoxicity on epithelial intestinal cells (FHs74Int) and on differentiated human hepatocellular carcinoma cells (HepaRG).[222] Interestingly, 4-dimethylaminopyridine (DMAP), 1,5,7triazabicyclo[4.4.0]dec-5-ene (TBD), and meta-(trimethylammonio)phenolate betaine ( $m$-BE) showed cytotoxicity against human hepatocellular carcinoma cells only at a high concentration.[222]

As also emphasized in this review, most of organo-catalyzed and organo-initiated polymerization reactions are based on the ring-opening of carbonyl-containing monomers such as, cyclic esters and cyclic carbonates. Nevertheless, few organic compounds, such as $N$ heterocyclic carbenes (NHCs) have allowed to broaden the scope of organocatalyzed polymerization with the ROP of cyclic ethers and siloxanes, the GTP of alkyl (meth)acrylates, as well as some step-growth polymerizations. So far, and to the best of our knowledge, the field of organopolymerization of less activated non-polar monomers, e.g. conjugated dienes or styrenics is still unexplored and could become one of the next challenges to be addressed. Only very recently, $\pi$-conjugated copolymers based on perfluoroaromatics and thiophene or alkynes moieties have been successfully synthesized in absence of any transition metals.[223, 224] The polymerization relies on the fluoride-catalyzed activation of $\mathrm{Si}-\mathrm{C}$ bonds on one hand and on nucleophilic aromatic substitutions on the other hand.

Spectacular achievements, such as the metal-free ring-opening metathesis polymerization (ROMP) of norbornene[225] and the metal-free atom transfer radical polymerization (ATRP) of methacrylates [226] and acrylonitrile, [227] have also been reported using organic photo-redox mediators. These two reactions that are traditionally catalyzed by transition metals could be promoted by electron transfer between the photo-sensitizer and the initiator upon light exposure. These recent examples attest to the exciting potential of metal-free techniques for the synthesis of a wide variety of functional polymeric materials. 


\section{Acknowledgments}

The authors are grateful to the Agence Nationale de la Recherche (ANR) - Programme Blanc (CATAPULT Project ANR-11-BS08-0011), and to the European Commission through project SUSPOL-EJD 642671 for financial support. 


\section{References}

[1] MacMillan DW. The advent and development of organocatalysis. Nature. 2008;455:304-8. [2] Wende RC, Schreiner PR. Evolution of asymmetric organocatalysis: multi- and retrocatalysis. Green Chem. 2012;14:1821-49.

[3] Bugaut X, Glorius F. Organocatalytic umpolung: N-heterocyclic carbenes and beyond. Chem Soc Rev. 2012;41:3511-22.

[4] Volla CMR, Atodiresei I, Rueping M. Catalytic C-C bond-forming multi-component cascade or domino reactions: pushing the boundaries of complexity in asymmetric organocatalysis. Chem Rev. 2013;114:2390-431.

[5] Dalko PI, editor. Comprehensive enantioselective organocatalysis: catalysts, reactions, and applications. Weinheim: Wiley-VCH Verlag GmbH \& Co; 2013; 1638 pp.

[6] Liu D, Chen EYX. Organocatalysis in biorefining for biomass conversion and upgrading. Green Chem. 2014;16:964-81.

[7] Kiesewetter MK, Shin EJ, Hedrick JL, Waymouth RM. Organocatalysis: opportunities and challenges for polymer synthesis. Macromolecules. 2010;43:2093-107.

[8] Dove AP. Organic catalysis for ring-opening polymerization. ACS Macro Letters. 2012;1:1409-12.

[9] Mespouille L, Coulembier O, Kawalec M, Dove AP, Dubois P. Implementation of metal-free ring-opening polymerization in the preparation of aliphatic polycarbonate materials. Prog Polym Sci. 2014;39:1144-64.

[10] Fuchise K, Chen YG, Satoh T, Kakuchi T. Recent progress in organocatalytic group transfer polymerization. Polym Chem. 2013;4:4278-91.

[11] Hirao A, Goseki R, Ishizone T. Advances in living anionic polymerization: from functional monomers, polymerization systems, to macromolecular architectures. Macromolecules. 2014;47:1883-905.

[12] Brown HA, Waymouth RM. Zwitterionic ring-opening polymerization for the synthesis of high molecular weight cyclic polymers. Acc Chem Res. 2013;46:2585-96.

[13] Thomas C, Bibal B. Hydrogen-bonding organocatalysts for ring-opening polymerization. Green Chem. 2014;16:1687-99.

[14] Fèvre M, Vignolle J, Gnanou Y, Taton D, Organocatalyzed ring-opening polymerizations. In Polymer science: a comprehensive reference, Vol. 4, Elsevier: Amsterdam, 2012. p. 67115.

[15] Fèvre M, Pinaud J, Gnanou Y, Vignolle J, Taton D. N-Heterocyclic carbenes (NHCs) as organocatalysts and structural components in metal-free polymer synthesis. Chem Soc Rev. 2013;42:2142-72.

[16] Naumann S, Dove AP. N-heterocyclic carbenes as organocatalysts for polymerizations: trends and frontiers. Polym Chem. 2015;6:3185-200.

[17] Zhang Y, Chen EYX. Conjugate-addition organopolymerization: rapid production of acrylic bioplastics by N-heterocyclic carbenes. Angew Chem Int Ed. 2012;51:2465-9.

[18] Naumann S, Schmidt FG, Schowner R, Frey W, Buchmeiser MR. Polymerization of methyl methacrylate by latent pre-catalysts based on $\mathrm{CO} 2$-protected $\mathrm{N}$-heterocyclic carbenes. Polym Chem. 2013;4:2731-40.

[19] Zhang Y, Schmitt M, Falivene L, Caporaso L, Cavallo L, Chen EYX. Organocatalytic conjugate-addition polymerization of linear and cyclic acrylic monomers by $\mathrm{N}$-heterocyclic carbenes: mechanisms of chain initiation, propagation, and termination. J Am Chem Soc. 2013;135:17925-42.

[20] Alsarraf J, Ammar YA, Robert F, Cloutet E, Cramail H, Landais Y. Cyclic guanidines as efficient organocatalysts for the synthesis of polyurethanes. Macromolecules. 2012;45:224956.

[21] Sardon H, Chan J, Ono R, Mecerreyes D, Hedrick J. Highly tunable polyurethanes: organocatalyzed polyaddition and subsequent post-polymerization modification of pentafluorophenyl ester sidechains. Polym Chem. 2014;5:3547-50.

[22] Liu N, Martin A, Robert F, Vincent J-M, Landais Y, Vignolle J, Cramail H, Taton D. Polyaldol synthesis by direct organocatalyzed crossed polymerization of bis(ketones) and bis(aldehydes). Macromolecules. 2014;47:525-33. 
[23] Xu T, Chen EYX. Probing site cooperativity of frustrated phosphine/borane Lewis pairs by a polymerization study. J Am Chem Soc. 2014;136:1774-7.

[24] Zhang S, Feret A, Lefebvre H, Tessier M, Fradet A. Poly(oxyalkylene) synthesis in Bronsted acid ionic liquids. Chem Commun. 2011;47:11092-4.

[25] Zhang S, Lemaire V, Feret A, Lefebvre H, Tessier M, Fradet A. Synthesis of linear and hyperbranched polyesters in Bronsted acid ionic liquids. Polym Chem. 2013;4:1538-45.

[26] Bordwell FG. Equilibrium acidities in dimethyl sulfoxide solution. Acc Chem Res. 1988;21:456-63.

[27] Raamat E, Kaupmees K, Ovsjannikov G, Trummal A, Kütt A, Saame J, Koppel I, Kaljurand I, Lipping L, Rodima T, Pihl V, Koppel IA, Leito I. Acidities of strong neutral Brønsted acids in different media. J Phys Org Chem. 2013;26:162-70.

[28] Ren JM, Fu Q, Blencowe A, Qiao GG. Organic catalyst-mediated ring-opening polymerization for the highly efficient synthesis of polyester-based star polymers. ACS Macro Letters. 2012;1:681-6.

[29] Gazeau-Bureau S, Delcroix D, Martín-Vaca B, Bourissou D, Navarro C, Magnet S. Organo-catalyzed ROP of $\varepsilon$-caprolactone: methanesulfonic acid competes with trifluoromethanesulfonic acid. Macromolecules. 2008;41:3782-4.

[30] Stanley N, Bucataru G, Miao Y, Favrelle A, Bria M, Stoffelbach F, Woisel P, Zinck P. Brønsted acid-catalyzed polymerization of $\varepsilon$-caprolactone in water: a mild and straightforward route to poly( $\varepsilon$-caprolactone)-graft-water-soluble polysaccharides. J Polym Sci, Part A: Polym Chem. 2014;52:2139-45.

[31] Basko M, Duda A, Kazmierski S, Kubisa P. Cationic copolymerization of racemic $\beta$ butyrolactone with L,L-lactide: one-pot synthesis of block copolymers. J Polym Sci, Part A: Polym Chem. 2013;51:4873-84.

[32] Couffin A, Martin-Vaca B, Bourissou D, Navarro C. Selective O-acyl ring-opening of betabutyrolactone catalyzed by trifluoromethane sulfonic acid: application to the preparation of well-defined block copolymers. Polym Chem. 2014;5:161-8.

[33] Foropoulos J, DesMarteau DD. Synthesis, properties, and reactions of bis((trifluoromethyl)sulfonyl) imide, (CF3SO2)2NH. Inorg Chem. 1984;23:3720-3.

[34] Makiguchi K, Kikuchi S, Satoh T, Kakuchi T. Synthesis of block and end-functionalized polyesters by triflimide-catalyzed ring-opening polymerization of $\varepsilon$-caprolactone, 1,5-dioxepan2-one, and rac-lactide. J Polym Sci, Part A: Polym Chem. 2013;51:2455-63.

[35] King JF, Acidity. In Sulphonic acids, esters and their derivatives (1991), John Wiley \& Sons, Ltd: Chichester, 2006. p. 249-59.

[36] Jin Y, Ji Y, He X, Kan S, Xia H, Liang B, Chen J, Wu H, Guo K, Li Z. oBenzenedisulfonimide as a recyclable cationic organocatalyst for the controlled/living ringopening polymerization of $\delta$-valerolactone and $\varepsilon$-caprolactone. Polym Chem. 2014;5:3098106.

[37] Pascual A, Leiza JR, Mecerreyes D. Acid catalyzed polymerization of macrolactones in bulk and aqueous miniemulsion: ring-opening vs. condensation. Eur Polym J. 2013;49:16019.

[38] Pascual A, Sardon H, Veloso A, Ruipérez F, Mecerreyes D. Organocatalyzed synthesis of aliphatic polyesters from ethylene brassylate: a cheap and renewable macrolactone. ACS Macro Letters. 2014;3:849-53.

[39] Delcroix D, Martin-Vaca B, Bourissou D, Navarro C. Ring-opening polymerization of trimethylene carbonate catalyzed by methanesulfonic acid: activated monomer versus active chain end mechanisms. Macromolecules. 2010;43:8828-35.

[40] Campos JM, Ribeiro MR, Ribeiro MF, Deffieux A, Peruch F. A new insight into the mechanism of the ring-opening polymerization of trimethylene carbonate catalyzed by methanesulfonic acid. Macromol Chem Phys. 2013;214:85-93.

[41] Campos JM, Ribeiro MR, Ribeiro MF, Deffieux A, Peruch F. Copolymerisation of $\varepsilon$ caprolactone and trimethylene carbonate catalysed by methanesulfonic acid. Eur Polym J. 2013;49:4025-34.

[42] Couffin A, Delcroix D, Martin-Vaca B, Bourissou D, Navarro C. Mild and efficient preparation of block and gradient copolymers by methanesulfonic acid catalyzed ring-opening polymerization of caprolactone and trimethylene carbonate. Macromolecules. 2013;46:435460. 
[43] Coady DJ, Horn HW, Jones GO, Sardon H, Engler AC, Waymouth RM, Rice JE, Yang YY, Hedrick JL. Polymerizing base sensitive cyclic carbonates using acid catalysis. ACS Macro Letters. 2013;2:306-12.

[44] Susperregui N, Delcroix D, Martin-Vaca B, Bourissou D, Maron L. Ring-opening polymerization of $\varepsilon$-caprolactone catalyzed by sulfonic acids: computational evidence for bifunctional activation. J Org Chem. 2010;75:6581-7.

[45] Engler AC, Chan JMW, Coady DJ, O'Brien JM, Sardon H, Nelson A, Sanders DP, Yang YY, Hedrick JL. Accessing new materials through polymerization and modification of a polycarbonate with a pendant activated ester. Macromolecules. 2013;46:1283-90.

[46] Sardon H, Engler AC, Chan JMW, Garcia JM, Coady DJ, Pascual A, Mecerreyes D, Jones GO, Rice JE, Horn HW, Hedrick JL. Organic acid-catalyzed polyurethane formation via a dualactivated mechanism: unexpected preference of $\mathrm{N}$-activation over $\mathrm{O}$-activation of isocyanates. J Am Chem Soc. 2013;135:16235-41.

[47] Ishihara K, Hasegawa A, Yamamoto H. Polystyrene-bound tetrafluorophenylbis(triflyl)methane as an organic-solvent-swellable and strong Brønsted acid catalyst. Angew Chem Int Ed. 2001;40:4077-9.

[48] Takada K, Fuchise K, Chen Y, Satoh T, Kakuchi T. Controlled polymerization of methyl acrylate for high-molecular-weight polymers by pentafluorophenylbis(triflyl)methane-promoted group transfer polymerization using triisopropylsilyl ketene acetal. J Polym Sci, Part A: Polym Chem. 2012;50:3560-6.

[49] Takada K, Fuchise K, Kubota N, Ito T, Chen Y, Satoh T, Kakuchi T. Synthesis of $\alpha-, \omega-$, and $\alpha, \omega$-end-functionalized poly(n-butyl acrylate)s by organocatalytic group transfer polymerization using functional initiator and terminator. Macromolecules. 2014;47:5514-25.

[50] Barker IA, Dove AP. Triarylsulfonium hexafluorophosphate salts as photoactivated acidic catalysts for ring-opening polymerisation. Chem Commun. 2013;49:1205-7.

[51] Balasubramanian A, Ku T-C, Shih H-P, Suman A, Lin H-J, Shih T-W, Han C-C. Chaingrowth cationic polymerization of 2-halogenated thiophenes promoted by Bronsted acids. Polym Chem. 2014;5:5928-41.

[52] Xu J, Song J, Pispas S, Zhang G. Controlled/living ring-opening polymerization of $\varepsilon$ caprolactone with salicylic acid as the organocatalyst. J Polym Sci, Part A: Polym Chem. 2014;52:1185-92.

[53] Christ P, Lindsay AG, Vormittag SS, Neudörfl J-M, Berkessel A, O'Donoghue AC. pKa values of chiral Brønsted acid catalysts: phosphoric acids/amides, sulfonyl/sulfuryl imides, and perfluorinated TADDOLs (TEFDDOLs). Chem Eur J. 2011;17:8524-8.

[54] Parmar D, Sugiono E, Raja S, Rueping M. Complete field guide to asymmetric BINOLphosphate derived bronsted acid and metal catalysis: history and classification by mode of activation; Bronsted acidity, hydrogen bonding, ion pairing, and metal phosphates. Chem Rev. 2014;114:9047-153.

[55] Akiyama T. Stronger Brönsted acids. Chem Rev. 2007;107:5744-58.

[56] Makiguchi K, Ogasawara Y, Kikuchi S, Satoh T, Kakuchi T. Diphenyl phosphate as an efficient acidic organocatalyst for controlled/living ring-opening polymerization of trimethylene carbonates leading to block, end-functionalized, and macrocyclic polycarbonates. Macromolecules. 2013;46:1772-82.

[57] Makiguchi K, Satoh T, Kakuchi T. Diphenyl phosphate as an efficient cationic organocatalyst for controlled/living ring-opening polymerization of $\delta$-valerolactone and $\varepsilon$ caprolactone. Macromolecules. 2011;44:1999-2005.

[58] Makiguchi K, Saito T, Satoh T, Kakuchi T. Bis(4-nitrophenyl) phosphate as an efficient organocatalyst for ring-opening polymerization of $\beta$-butyrolactone leading to endfunctionalized and diblock polyesters. J Polym Sci, Part A: Polym Chem. 2014;52:2032-9.

[59] Werner T. Phosphonium salt organocatalysis. Adv Synth Catal. 2009;351:1469-81.

[60] Terada M, Momiyama N, Phosphoric acid catalysis of reactions not involving imines. In Science of synthesis, asymmetric organocatalysis 2, Brønsted base and acid catalysts, and additional topics, Georg Thieme Verlag KG: New York, 2012. p. 219-78.

[61] Miao Y, Phuphuak Y, Rousseau C, Bousquet T, Mortreux A, Chirachanchai S, Zinck P. Ring-opening polymerization of lactones using binaphthyl-diyl hydrogen phosphate as organocatalyst and resulting monosaccharide functionalization of polylactones. J Polym Sci, Part A: Polym Chem. 2013;51:2279-87. 
[62] Miao Y, Mortreux A, Zinck P. Polyester functionalized carbohydrates via organocatalyzed ring-opening polymerization. Carbohydr Chem. 2014;40:298-311.

[63] Makiguchi K, Yamanaka T, Kakuchi T, Terada M, Satoh T. Binaphthol-derived phosphoric acids as efficient chiral organocatalysts for the enantiomer-selective polymerization of raclactide. Chem Commun. 2014;50:2883-5.

[64] Kan S, Jin Y, He X, Chen J, Wu H, Ouyang P, Guo K, Li Z. Imidodiphosphoric acid as a bifunctional catalyst for the controlled ring-opening polymerization of $\delta$-valerolactone and $\varepsilon$ caprolactone. Polym Chem. 2013;4:5432-9.

[65] He X, Ji Y, Jin Y, Kan S, Xia H, Chen J, Liang B, Wu H, Guo K, Li Z. Bifunctional imidodiphosphoric acid-catalyzed controlled/living ring-opening polymerization of trimethylene carbonate resulting block, $\alpha, \omega$-dihydroxy telechelic, and star-shaped polycarbonates. J Polym Sci, Part A: Polym Chem. 2014;52:1009-19.

[66] Ishikawa T, editor. Superbases for organic synthesis: guanidines, amidines and phosphazenes and related organocatalysts. Chichester: John Wiley \& Sons, Ltd; 2009; 336 pp.

[67] Schwesinger R, Schlemper H. Peralkylated polyaminophosphazenes-extremely strong, neutral nitrogen bases. Angew Chem Int Ed. 1987;26:1167-9.

[68] Boileau S, Illy N. Activation in anionic polymerization: why phosphazene bases are very exciting promoters. Prog Polym Sci. 2011;36:1132-51.

[69] Zhao JP, Alamri H, Hadjichristidis N. A facile metal-free "grafting-from" route from acrylamide-based substrate toward complex macromolecular combs. Chem Commun. 2013;49:7079-81.

[70] Zhao J, Pahovnik D, Gnanou Y, Hadjichristidis N. Phosphazene-promoted metal-free ringopening polymerization of ethylene oxide initiated by carboxylic acid. Macromolecules. 2014;47:1693-8.

[71] Zhao J, Pahovnik D, Gnanou Y, Hadjichristidis N. A "catalyst switch" strategy for the sequential metal-free polymerization of epoxides and cyclic esters/carbonate. Macromolecules. 2014;47:3814-22.

[72] Zhao J, Pahovnik D, Gnanou Y, Hadjichristidis N. One-pot synthesis of linear- and threearm star-tetrablock quarterpolymers via sequential metal-free ring-opening polymerization using a "catalyst switch" strategy. J Polym Sci, Part A: Polym Chem. 2015;53:304-12.

[73] Hadjichristidis N, Zhao J, Pahovnik D, Gnanou Y. Sequential polymerization of ethylene oxide, $\varepsilon$-caprolactone and L-lactide: a one-pot metal-free route to tri- and pentablock terpolymers. Polym Chem. 2014;5:3750-3.

[74] Alamri H, Zhao J, Pahovnik D, Hadjichristidis N. Phosphazene-catalyzed ring-opening polymerization of $\varepsilon$-caprolactone: influence of solvents and initiators. Polym Chem. 2014;5:5471-8.

[75] Guerin W, Helou M, Carpentier J-F, Slawinski M, Brusson J-M, Guillaume SM. Macromolecular engineering via ring-opening polymerization (1): L-lactide/trimethylene carbonate block copolymers as thermoplastic elastomers. Polym Chem. 2013;4:1095-106.

[76] Samuel C, Chalamet Y, Boisson F, Majeste J-C, Becquart F, Fleury E. Highly efficient metal-free organic catalysts to design new environmental-friendly starch-based blends. $J$ Polym Sci, Part A: Polym Chem. 2014;52:493-503.

[77] Illy N, Boileau S, Winnik MA, Penelle J, Barbier V. Thiol-ene "clickable" carbon-chain polymers based on diallyl cyclopropane-1,1-dicarboxylate. Polymer. 2012;53:903-12.

[78] Yang HJ, Xu JB, Pispas S, Zhang GZ. Hybrid copolymerization of $\varepsilon$-caprolactone and methyl methacrylate. Macromolecules. 2012;45:3312-7.

[79] Xu JB, Yang HJ, Zhang GZ. Synthesis of poly( $\varepsilon$-caprolactone-co-methacrylic acid) copolymer via phosphazene-catalyzed hybrid copolymerization. Macromol Chem Phys. 2013;214:378-85.

[80] Yang H, Xu J, Pispas S, Zhang G. One-step synthesis of hyperbranched biodegradable polymer. RSC Adv. 2013;3:6853-8.

[81] De Winter J, Dove AP, Knoll A, Gerbaux P, Dubois P, Coulembier O. Control over molar mass, dispersity, end-groups and kinetics in cyclopolymerization of ortho-phthalaldehyde: adapted choice of a phosphazene organocatalyst. Polym Chem. 2014;5:706-11.

[82] Köstler S. Polyaldehydes: homopolymers, block copolymers and promising applications. Polym Int. 2012;61:1221-7. 
[83] Ito $\mathrm{H}$, Willson CG. Chemical amplification in the design of dry developing resist materials. Polym Eng Sci. 1983;23:1012-8.

[84] Methot JL, Roush WR. Nucleophilic phosphine organocatalysis. Adv Synth Catal. 2004;346:1035-50.

[85] Marinetti A, Voituriez A. Enantioselective phosphine organocatalysis. Synlett. 2010:17494.

[86] Myers M, Connor EF, Glauser T, Mock A, Nyce G, Hedrick JL. Phosphines: nucleophilic organic catalysts for the controlled ring-opening polymerization of lactides. J Polym Sci, Part A: Polym Chem. 2002;40:844-51.

[87] Fèvre M, Vignolle J, Heroguez V, Taton D. Tris(2,4,6-trimethoxyphenyl)phosphine (TTMPP) as potent organocatalyst for group transfer polymerization of alkyl (meth)acrylates. Macromolecules. 2012;45:7711-8.

[88] Arduengo AJ, Harlow RL, Kline M. A stable crystalline carbene. J Am Chem Soc. 1991;113:361-3.

[89] Bourissou D, Guerret O, Gabbai FP, Bertrand G. Stable carbenes. Chem Rev. 2000;100:39-91.

[90] Nair V, Bindu S, Sreekumar V. N-heterocyclic carbenes: reagents, not just ligands! Angew Chem Int Ed. 2004;43:5130-5.

[91] Hahn FE, Jahnke MC. Heterocyclic carbenes: synthesis and coordination chemistry. Angew Chem Int Ed. 2008;47:3122-72.

[92] Dröge T, Glorius F. The measure of all rings- N-heterocyclic carbenes. Angew Chem Int Ed. 2010;49:6940-52.

[93] Hopkinson MN, Richter C, Schedler M, Glorius F. An overview of N-heterocyclic carbenes. Nature. 2014;510:485-96.

[94] Magill AM, Cavell KJ, Yates BF. Basicity of nucleophilic carbenes in aqueous and nonaqueous solvents-theoretical predictions. J Am Chem Soc. 2004;126:8717-24.

[95] Higgins EM, Sherwood JA, Lindsay AG, Armstrong J, Massey RS, Alder RW, O'Donoghue AC. pKas of the conjugate acids of $\mathrm{N}$-heterocyclic carbenes in water. Chem Commun. 2011;47:1559-61.

[96] Fischer C, Smith SW, Powell DA, Fu GC. Umpolung of Michael acceptors catalyzed by Nheterocyclic carbenes. J Am Chem Soc. 2006;128:1472-3.

[97] Biju AT, Padmanaban M, Wurz NE, Glorius F. N-heterocyclic carbene catalyzed umpolung of Michael acceptors for intermolecular reactions. Angew Chem Int Ed. 2011;50:8412-5.

[98] Ryan SJ, Candish L, Lupton DW. Acyl anion free N-heterocyclic carbene organocatalysis. Chem Soc Rev. 2013;42:4906-17.

[99] Mahatthananchai J, Bode JW. On the mechanism of N-heterocyclic carbene-catalyzed reactions involving acyl azoliums. Acc Chem Res. 2014;47:696-707.

[100] Chauhan P, Enders D. N-heterocyclic carbene catalyzed activation of esters: a new option for asymmetric domino reactions. Angew Chem Int Ed. 2014;53:1485-7.

[101] Diez-Gonzalez S, Marion N, Nolan SP. N-heterocyclic carbenes in late transition metal catalysis. Chem Rev. 2009;109:3612-76.

[102] Nelson DJ, Nolan SP. Quantifying and understanding the electronic properties of Nheterocyclic carbenes. Chem Soc Rev. 2013;42:6723-53.

[103] Herrmann WA. N-heterocyclic carbenes: A new concept in organometallic catalysis. Angew Chem Int Ed. 2002;41:1290-309.

[104] Breslow R. On the mechanism of thiamine action. IV. Evidence from studies on model systems. J Am Chem Soc. 1958;80:3719-26.

[105] Grossmann A, Enders D. N-heterocyclic carbene catalyzed domino reactions. Angew Chem Int Ed. 2012;51:314-25.

[106] Moore JL, Rovis T. Carbene catalysts. Top Curr Chem. 2010;291:77-144.

[107] Marion N, Diez-Gonzalez S, Nolan SP. N-heterocyclic carbenes as organocatalysts. Angew Chem Int Ed. 2007;46:2988-3000.

[108] Enders D, Niemeier O, Henseler A. Organocatalysis by N-heterocyclic, carbenes. Chem Rev. 2007;107:5606-55.

[109] Nair V, Vellalath S, Babu BP. Recent advances in carbon-carbon bond-forming reactions involving homoenolates generated by NHC catalysis. Chem Soc Rev. 2008;37:2691-8.

[110] Kamber NE, Jeong W, Waymouth RM, Pratt RC, Lohmeijer BGG, Hedrick JL. Organocatalytic ring-opening polymerization. Chem Rev. 2007;107:5813-40. 
[111] Naumann S, Buchmeiser M. Liberation of $\mathrm{N}$-heterocyclic carbenes (NHCs) from thermally labile progenitors: protected NHCs as versatile tools in organo- and polymerization catalysis. Catal Sci Technol. 2014;4:2466-79.

[112] Arduengo AJ, Dias HVR, Harlow RL, Kline M. Electronic stabilization of nucleophilic carbenes. J Am Chem Soc. 1992;114:5530-4.

[113] Orsini M, Chiarotto I, Sotgiu G, Inesi A. Polarity reversal induced by electrochemically generated thiazol-2-ylidenes: the Stetter reaction. Electrochim Acta. 2010;55:3511-7.

[114] Orsini M, Chiarotto I, Feeney MMM, Feroci M, Sotgiu G, Inesi A. Umpolung of $\alpha, \beta-$ unsaturated aldehydes by electrogenerated $\mathrm{NHCs}$ in ionic liquids: synthesis of $\mathrm{Y}$ butyrolactones. Electrochem Commun. 2011;13:738-41.

[115] Kuhn N, Kratz T. Synthesis of imidazol-2-ylidenes by reduction of imidazole-2(3H)thiones. Synthesis. 1993:561-2.

[116] Wang Y, Zhang L, Guo X, Zhang R, Li J. Characteristics and mechanism of L-lactide polymerization using N-heterocyclic carbene organocatalyst. J Polym Res. 2013;20:1-6.

[117] Zhang R, Zhang L, Wang J, Guo X. Ring-opening copolymerization of $\varepsilon$-caprolactone with 2,2-dimethyltrimethylene carbonate using $\mathrm{N}$-heterocyclic carbene organocatalysts. Polym Bull. 2013;70:1289-301.

[118] Xia H, Kan S, Li Z, Chen J, Cui S, Wu W, Ouyang P, Guo K. N-heterocyclic carbenes as organocatalysts in controlled/living ring-opening polymerization of O-carboxyanhydrides derived from L-lactic acid and L-mandelic acid. J Polym Sci, Part A: Polym Chem. 2014;52:2306-15.

[119] Wanzlick HW. Aspects of nucleophilic carbene chemistry. Angew Chem Int Ed. 1962;1:75-80.

[120] Coutelier O, El Ezzi M, Destarac M, Bonnette F, Kato T, Baceiredo A, Sivasankarapillai G, Gnanou Y, Taton D. N-Heterocyclic carbene-catalysed synthesis of polyurethanes. Polym Chem. 2012;3:605-8.

[121] Duong HA, Cross MJ, Louie J. N-heterocyclic carbenes as highly efficient catalysts for the cyclotrimerization of isocyanates. Org Lett. 2004;6:4679-81.

[122] Hoppe A, Sadaka F, Brachais CH, Boni G, Couvercelle JP, Plasseraud L. n-Butyl-3methylimidazolium-2-carboxylate: a versatile precatalyst for the ring-opening polymerization of $\varepsilon$-caprolactone and rac-lactide under solvent-free conditions. Beilstein $\mathrm{J}$ Org Chem. 2013;9:647-54.

[123] Fèvre M, Vignolle J, Taton D. Azolium hydrogen carbonates and azolium carboxylates as organic pre-catalysts for $\mathrm{N}$-heterocyclic carbene-catalysed group transfer and ring-opening polymerisations. Polym Chem. 2013;4:1995-2003.

[124] Naumann S, Schmidt FG, Frey W, Buchmeiser MR. Protected N-heterocyclic carbenes as latent pre-catalysts for the polymerization of $\varepsilon$-caprolactone. Polym Chem. 2013;4:4172-81. [125] Lindner R, Lejkowski ML, Lavy S, Deglmann P, Wiss KT, Zarbakhsh S, Meyer L, Limbach $\mathrm{M}$. Ring-opening polymerization and copolymerization of propylene oxide catalyzed by $\mathrm{N}$ heterocyclic carbenes. ChemCatChem. 2014;6:618-25.

[126] Raynaud J, Nzahou Ottou W, Gnanou Y, Taton D. Metal-free and solvent-free access to $\alpha, \omega$-heterodifunctionalized poly(propylene oxide)s by $\mathrm{N}$-heterocyclic carbene-induced ring opening polymerization. Chem Commun. 2010;46:3203-5.

[127] Reitz A, Wilhelm R, Kuckling D. Ring-opening polymerization of organic carbonates using CO2-carbene adducts as effective organo catalyst. Macromol Symp. 2013;334:92-7.

[128] Fèvre M, Pinaud J, Leteneur A, Gnanou Y, Vignolle J, Taton D, Miqueu K, Sotiropoulos $\mathrm{JM}$. Imidazol(in)ium hydrogen carbonates as a genuine source of $\mathrm{N}$-heterocyclic carbenes (NHCs): applications to the facile preparation of $\mathrm{NHC}$ metal complexes and to $\mathrm{NHC}$ organocatalyzed molecular and macromolecular syntheses. J Am Chem Soc. 2012;134:677684.

[129] Neilson BM, Bielawski CW. Photoswitchable NHC-promoted ring-opening polymerizations. Chem Commun. 2013;49:5453-5.

[130] Murugan R, Scriven EFV. Applications of dialkylaminopyridine (DMAP) catalysts in organic synthesis. Aldrichim Acta. 2003;36:21-7.

[131] Miao Y, Rousseau C, Mortreux A, Martin P, Zinck P. Access to new carbohydratefunctionalized polylactides via organocatalyzed ring-opening polymerization. Polymer. 2011;52:5018-26. 
[132] Mingfa $Y$, Tao W, Na Q, Yongkai H, Daping Q. Controllable ring-opening polymerization of trimethylene carbonate catalyzed by aliphatic tertiary amines in the presence of benzyl alcohol or F127. Polym Int. 2012;61:1525-31.

[133] Coady DJ, Engler AC, Horn HW, Bajjuri KM, Fukushima K, Jones GO, Nelson A, Rice $\mathrm{JE}$, Hedrick JL. Catalyst chelation effects in organocatalyzed ring-opening polymerization of lactide. ACS Macro Letters. 2012;1:19-22.

[134] Kaljurand I, Kutt A, Soovali L, Rodima T, Maemets V, Leito I, Koppel IA. Extension of the self-consistent spectrophotometric basicity scale in acetonitrile to a full span of 28 pKa units: unification of different basicity scales. J Org Chem. 2005;70:1019-28.

[135] Lohmeijer BGG, Pratt RC, Leibfarth F, Logan JW, Long DA, Dove AP, Nederberg F, Choi J, Wade C, Waymouth RM, Hedrick JL. Guanidine and Amidine Organocatalysts for RingOpening Polymerization of Cyclic Esters. Macromolecules. 2006;39:8574-83.

[136] Coulembier O, Lemaur V, Josse T, Minoia A, Cornil J, Dubois P. Synthesis of poly(Llactide) and gradient copolymers from a L-lactide/trimethylene carbonate eutectic melt. Chem Sci. 2012;3:723-6.

[137] Venkataraman S, Hedrick JL, Yang YY. Fluorene-functionalized aliphatic polycarbonates: design, synthesis and aqueous self-assembly of amphiphilic block copolymers. Polym Chem. 2014;5:2035-40.

[138] Zhao Z, Wang J, Mao H-Q, Leong KW. Polyphosphoesters in drug and gene delivery. Adv Drug Delivery Rev. 2003;55:483-99.

[139] Iwasaki Y, Yamaguchi E. Synthesis of well-defined thermoresponsive polyphosphoester macroinitiators using organocatalysts. Macromolecules. 2010;43:2664-6.

[140] Zhang S, Li A, Zou J, Lin LY, Wooley KL. Facile synthesis of clickable, water-soluble, and degradable polyphosphoesters. ACS Macro Letters. 2012;1:328-33.

[141] Clément B, Grignard B, Koole L, Jérôme C, Lecomte P. Metal-free strategies for the synthesis of functional and well-defined polyphosphoesters. Macromolecules. 2012;45:447686.

[142] Lim YH, Heo GS, Cho S, Wooley KL. Construction of a reactive diblock copolymer, polyphosphoester-block-poly(L-lactide), as a versatile framework for functional materials that are capable of full degradation and nanoscopic assembly formation. ACS Macro Letters. 2013;2:785-9.

[143] Martello MT, Burns A, Hillmyer M. Bulk ring-opening transesterification polymerization of the renewable $\delta$-decalactone using an organocatalyst. ACS Macro Letters. 2012;1:131-5.

[144] Guillerm B, Lemaur V, Ernould B, Cornil J, Lazzaroni R, Gohy J-F, Dubois P, Coulembier $O$. A one-pot two-step efficient metal-free process for the generation of PEO-b-PCL-b-PLA amphiphilic triblock copolymers. RSC Adv. 2014;4:10028-38.

[145] Appel EA, Lee VY, Nguyen TT, McNeil M, Nederberg F, Hedrick JL, Swope WC, Rice JE, Miller RD, Sly J. Toward biodegradable nanogel star polymers via organocatalytic ROP. Chem Commun. 2012;48:6163-5.

[146] Kim H, Olsson JV, Hedrick JL, Waymouth RM. Facile Synthesis of Functionalized Lactones and Organocatalytic Ring-Opening Polymerization. ACS Macro Letters. 2012;1:8457.

[147] Silvers AL, Chang CC, Emrick T. Functional aliphatic polyesters and nanoparticles prepared by organocatalysis and orthogonal grafting chemistry. J Polym Sci, Part A: Polym Chem. 2012;50:3517-29.

[148] Bouyahyi M, Pepels MPF, Heise A, Duchateau R. $\omega$-Pentandecalactone polymerization and $\omega$-pentadecalactone/ $\varepsilon$-caprolactone copolymerization reactions using organic catalysts. Macromolecules. 2012;45:3356-66.

[149] Castillo JA, Borchmann DE, Cheng AY, Wang Y, Hu C, Garcia AJ, Weck M. Well-defined poly(lactic acid)s containing poly(ethylene glycol) side chains. Macromolecules. 2012;45:62-9. [150] Zhang S, Zou J, Zhang F, Elsabahy M, Felder SE, Zhu J, Pochan DJ, Wooley KL. Rapid and versatile construction of diverse and functional nanostructures derived from a polyphosphoester-based biomimetic block copolymer system. $\mathrm{J}$ Am Chem Soc. 2012;134:18467-74.

[151] Guerin W, Helou M, Slawinski M, Brusson JM, Guillaume SM, Carpentier JF. Macromolecular engineering via ring-opening polymerization (2): L-lactide/trimethylene carbonate copolymerization-kinetic and microstructural control via catalytic tuning. Polym Chem. 2013;4:3686-93. 
[152] Guerin W, Diallo AK, Kirilov E, Helou M, Slawinski M, Brusson J-M, Carpentier J-F, Guillaume SM. Enantiopure isotactic PCHC synthesized by ring-opening polymerization of cyclohexene carbonate. Macromolecules. 2014;47:4230-5.

[153] Mikami K, Lonnecker AT, Gustafson TP, Zinnel NF, Pai PJ, Russell DH, Wooley KL. Polycarbonates derived from glucose via an organocatalytic approach. J Am Chem Soc. 2013:135:6826-9.

[154] Coulembier O, Moins Sb, Todd R, Dubois P. External and reversible CO2 regulation of ring-opening polymerizations based on a primary alcohol propagating species. Macromolecules. 2014;47:486-91.

[155] Xu Q, Sardon H, Chan JMW, Hedrick JL, Yang YY. Polyurethane-coated silica particles with broad-spectrum antibacterial properties. Polym Chem. 2015;6:2011-22.

[156] Maisonneuve L, Lebarbe T, Grau E, Cramail H. Structure-properties relationship of fatty acid-based thermoplastics as synthetic polymer mimics. Polym Chem. 2013;4:5472-517.

[157] Lambeth $\mathrm{RH}$, Henderson TJ. Organocatalytic synthesis of (poly)hydroxyurethanes from cyclic carbonates and amines. Polymer (United Kingdom). 2013;54:5568-73.

[158] Fukushima K, Coady DJ, Jones GO, Almegren HA, Alabdulrahman AM, Alsewailem FD, Horn HW, Rice JE, Hedrick JL. Unexpected efficiency of cyclic amidine catalysts in depolymerizing poly(ethylene terephthalate). J Polym Sci, Part A: Polym Chem. 2013;51:160611.

[159] Horn HW, Jones GO, Wei DS, Fukushima K, Lecuyer JM, Coady DJ, Hedrick JL, Rice JE. Mechanisms of organocatalytic amidation and trans-esterification of aromatic esters as a model for the depolymerization of poly(ethylene) terephthalate. J Phys Chem $A$. 2012;116:12389-98.

[160] Fukushima K, Lecuyer JM, Wei DS, Horn HW, Jones GO, Al-Megren HA, Alabdulrahman AM, Alsewailem FD, McNeil MA, Rice JE, Hedrick JL. Advanced chemical recycling of poly(ethylene terephthalate) through organocatalytic aminolysis. Polym Chem. 2013;4:16106.

[161] Zhang Z, Schreiner PR. (Thio)urea organocatalysis - what can be learnt from anion recognition? Chem Soc Rev. 2009;38:1187-98.

[162] Stevens DM, Tempelaar S, Dove AP, Harth E. Nanosponge formation from organocatalytically synthesized poly(carbonate) copolymers. ACS Macro Letters. 2012;1:9158.

[163] Todd R, Rubio G, Hall DJ, Tempelaar S, Dove AP. Benzyl bispidine as an efficient replacement for (-)-sparteine in ring-opening polymerisation. Chem Sci. 2013;4:1092-7.

[164] Tempelaar S, Barker IA, Truong VX, Hall DJ, Mespouille L, Dubois P, Dove AP. Organocatalytic synthesis and post-polymerization functionalization of propargyl-functional poly(carbonate)s. Polym Chem. 2013;4:174-83.

[165] Brannigan RP, Walder A, Dove AP. Block copolymer materials from the organocatalytic ring-opening polymerization of a pentaerythritol-derived cyclic carbonate. J Polym Sci, Part A: Polym Chem. 2014;52:2279-86.

[166] Venkataraman S, Veronica N, Voo ZX, Hedrick JL, Yang YY. 2-Amino-1,3-propane diols: a versatile platform for the synthesis of aliphatic cyclic carbonate monomers. Polym Chem. 2013;4:2945-8.

[167] Edward JA, Kiesewetter MK, Kim H, Flanagan JCA, Hedrick JL, Waymouth RM. Organocatalytic synthesis of quinine-functionalized poly(carbonate)s. Biomacromolecules. 2012;13:2483-9.

[168] Tempelaar S, Mespouille L, Dubois P, Dove AP. Organocatalytic synthesis and postpolymerization functionalization of allyl-functional poly(carbonate)s. Macromolecules. 2011;44:2084-91.

[169] Aguirre-Chagala YE, Santos JL, Herrera-Najera R, Herrera-Alonso M. Organocatalytic copolymerization of a cyclic carbonate bearing protected 2,2-bis(hydroxymethyl) groups and D,L-lactide. Effect of hydrophobic block chemistry on nanoparticle properties. Macromolecules. 2013;46:5871-81.

[170] Blake TR, Waymouth RM. Organocatalytic ring-opening polymerization of morpholinones: new strategies to functionalized polyesters. J Am Chem Soc. 2014;136:92525.

[171] Thomas C, Peruch F, Bibal B. Ring-opening polymerization of lactones using supramolecular organocatalysts under simple conditions. RSC Adv. 2012;2:12851-6. 
[172] Coady DJ, Fukushima K, Horn HW, Rice JE, Hedrick JL. Catalytic insights into acid/base conjugates: highly selective bifunctional catalysts for the ring-opening polymerization of lactide. Chem Commun. 2011;47:3105-7.

[173] Makiguchi K, Kikuchi S, Yanai K, Ogasawara Y, Sato S-i, Satoh T, Kakuchi T. Diphenyl phosphate/4-dimethylaminopyridine as an efficient binary organocatalyst system for controlled/living ring-opening polymerization of L-lactide leading to diblock and endfunctionalized poly(L-lactide)s. J Polym Sci, Part A: Polym Chem. 2014;52:1047-54.

[174] Miao Y, Stanley N, Favrelle A, Bousquet T, Bria M, Mortreux A, Zinck P. New acid/base salts as co-catalysts for the organocatalyzed ring-opening polymerization of lactide. J Polym Sci, Part A: Polym Chem. 2015;53:659-64.

[175] Kadota J, Pavlovic D, Hirano H, Okada A, Agari Y, Bibal B, Deffieux A, Peruch F. Controlled bulk polymerization of L-lactide and lactones by dual activation with organo-catalytic systems. RSC Adv. 2014;4:14725-32.

[176] Coulembier O, Josse T, Guillerm B, Gerbaux P, Dubois P. An imidazole-based organocatalyst designed for bulk polymerization of lactide isomers: inspiration from Nature. Chem Commun. 2012;48:11695-7.

[177] Guillerm B, Lemaur V, Cornil J, Lazzaroni R, Dubois P, Coulembier O. Ammonium betaines: efficient ionic nucleophilic catalysts for the ring-opening polymerization of L-lactide and cyclic carbonates. Chem Commun. 2014;50:10098-101.

[178] Wang X, Cui S, Li Z, Kan S, Zhang Q, Zhao C, Wu H, Liu J, Wu W, Guo K. A baseconjugate-acid pair for living/controlled ring-opening polymerization of trimethylene carbonate through hydrogen-bonding bifunctional synergistic catalysis. Polym Chem. 2014;5:6051-9.

[179] Kusumoto S, Ito S, Nozaki K. Direct aldol polymerization of acetaldehyde with organocatalyst/Bronsted acid systems. Asian J Org Chem. 2013;2:977-82.

[180] Liu N, Vignolle J, Vincent J-M, Robert F, Landais Y, Cramail H, Taton D. One-pot synthesis and PEGylation of hyperbranched polyacetals with a degree of branching of $100 \%$. Macromolecules. 2014;47:1532-42.

[181] Chatterjee S, Ramakrishnan S. Hyperbranched polyacetals with tunable degradation rates. Macromolecules. 2011;44:4658-64.

[182] Chatterjee S, Ramakrishnan S. Defect-free hyperbranched polydithioacetal via melt polymerization. ACS Macro Letters. 2012;1:593-8.

[183] Martin A, Robert F, Taton D, Cramail H, Vincent J-M, Landais Y. Organocatalyzed stepgrowth polymerization through desymmetrization of cyclic anhydrides: synthesis of chiral polyesters. Chem Eur J. 2014;20:11946-53.

[184] Goldys AM, Dixon DJ. Organocatalytic ring-opening polymerization of cyclic esters mediated by highly active bifunctional iminophosphorane catalysts. Macromolecules. 2014;47:1277-84.

[185] Prasad AV, Stubbs LP, Ma Z, Yinghuai Z. Zwitterionic ring opening polymerization of lactide by metal free catalysts: production of cyclic polymers. J Appl Polym Sci. 2012;123:1568-75.

[186] Vishwa Prasad A, Yinghuai Z. Syntheses of cyclic poly(lactones) by zwitterionic ringopening polymerization catalyzed by $\mathrm{N}$-heterocyclic carbene. J Appl Polym Sci. 2013;128:3411-6.

[187] Zhang L, Wang Z, Wang J, Wang Y, Liu C. Unsymmetrical N-heterocyclic carbene: 1isopropyl-3-benzylimidazol-2- ylidene as catalyst for ring-opening polymerization of $\varepsilon$ caprolactone. J Macromol Sci, Pure Appl Chem. 2012;49:528-32.

[188] Brown HA, Xiong S, Medvedev GA, Chang YA, Abu-Omar MM, Caruthers JM, Waymouth RM. Zwitterionic ring-opening polymerization: models for kinetics of cyclic poly(caprolactone) synthesis. Macromolecules. 2014;47:2955-63.

[189] Acharya AK, Chang YA, Jones GO, Rice JE, Hedrick JL, Horn HW, Waymouth RM. Experimental and computational studies on the mechanism of zwitterionic ring-opening polymerization of $\delta$-valerolactone with $\mathrm{N}$-heterocyclic carbenes. J Phys Chem $\mathrm{B}$. 2014;118:6553-60.

[190] Brown HA, Chang YA, Waymouth RM. Zwitterionic polymerization to generate high molecular weight cyclic poly(carbosiloxane)s. J Am Chem Soc. 2013;135:18738-41.

[191] Stukenbroeker TS, Solis-Ibarra D, Waymouth RM. Synthesis and topological trapping of cyclic poly(alkylene phosphates). Macromolecules. 2014;47:8224-30. 
[192] Guo L, Lahasky SH, Ghale K, Zhang D. N-heterocyclic carbene-mediated zwitterionic polymerization of $\mathrm{N}$-substituted $\mathrm{N}$-carboxyanhydrides toward poly(a-peptoid)s: kinetic, mechanism, and architectural control. J Am Chem Soc. 2012;134:9163-71.

[193] Nzahou Ottou W, Bourichon D, Vignolle J, Wirotius A-L, Robert F, Landais Y, Sotiropoulos J-M, Miqueu K, Taton D. Cyclodimerization versus polymerization of methyl methacrylate induced by $\mathrm{N}$-heterocyclic carbenes: a combined experimental and theoretical study. Chem Eur J. 2014;20:3989-97.

[194] Hong M, Chen EYX. Proton-transfer polymerization (HTP): converting methacrylates to polyesters by an N-heterocyclic carbene. Angew Chem Int Ed. 2014;53:11900-6.

[195] Matsuoka S-I, Namera S, Suzuki M. Oxa-Michael addition polymerization of acrylates catalyzed by N-heterocyclic carbenes. Polym Chem. 2015;6:294-301.

[196] Holdroyd RS, Page MJ, Warren MR, Whittlesey MK. Intramolecular C-H insertion in ringexpanded N-heterocyclic carbenes. Tetrahedron Lett. 2010;51:557-9.

[197] Naumann S, Epple S, Bonten C, Buchmeiser MR. Polymerization of $\varepsilon$-caprolactam by latent precatalysts based on protected $\mathrm{N}$-heterocyclic carbenes. ACS Macro Letters. 2013;2:609-12.

[198] Wichterle O, Sebenda J, Kralicek J, The anionic polymerization of caprolactam. In Adv Polym Sci, Vol. 2/4, Springer Berlin Heidelberg: 1961. p. 578-95.

[199] Mikheyev VN, Kharitonov VM, Sokolova NP. Chain transfer in anionic polymerization of caprolactam. Polymer Science USSR. 1979;21:2121-8.

[200] Naumann S, Schmidt FG, Speiser M, Böhl M, Epple S, Bonten C, Buchmeiser MR. Anionic ring-opening homo- and copolymerization of lactams by latent, protected $\mathrm{N}$ heterocyclic carbenes for the preparation of PA 12 and PA 6/12. Macromolecules. 2013;46:8426-33.

[201] Brule E, Guerineau V, Vermaut P, Prima F, Balogh J, Maron L, Slawin AMZ, Nolan SP, Thomas CM. Polymerization of cyclic esters using N-heterocyclic carbene carboxylate catalysts. Polym Chem. 2013;4:2414-23.

[202] Naumann S, Speiser M, Schowner R, Giebel E, Buchmeiser MR. Air stable and latent single-component curing of epoxy/anhydride resins catalyzed by thermally liberated $\mathrm{N}$ heterocyclic carbenes. Macromolecules. 2014;47:4548-56.

[203] Buchmeiser MR, Kammerer JA, Naumann S, Unold J, Ghomeshi R, Selvarayan SK, Weichand P, Gadow R. Latent CO2-protected N-heterocyclic carbene-based singlecomponent system-derived epoxy/glass fiber composites. Macromol Mater Eng. 2015:DOI: 10.1002/mame.201500090.

[204] Brown HA, De Crisci AG, Hedrick JL, Waymouth RM. Amidine-mediated zwitterionic polymerization of lactide. ACS Macro Letters. 2012;1:1113-5.

[205] Culkin DA, Jeong W, Csihony S, Gomez ED, Balsara NP, Hedrick JL, Waymouth RM. Zwitterionic polymerization of lactide to cyclic poly(lactide) by using $\mathrm{N}$-heterocyclic carbene organocatalysts. Angew Chem Int Ed. 2007;46:2627-30.

[206] Zhang X, Waymouth RM. Zwitterionic ring-opening polymerization with isothioureas. ACS Macro Letters. 2014;3:1024-8.

[207] Jaffredo CG, Carpentier JF, Guillaume SM. Controlled ROP of $\beta$-butyrolactone simply mediated by amidine, guanidine, and phosphazene organocatalysts. Macromol Rapid Commun. 2012;33:1938-44.

[208] Jaffredo CG, Carpentier JF, Guillaume SM. Organocatalyzed controlled ROP of $\beta$ lactones towards poly(hydroxyalkanoate)s: from $\beta$-butyrolactone to benzyl $\beta$-malolactone polymers. Polym Chem. 2013;4:3837-50.

[209] Jaffredo CG, Carpentier JF, Guillaume SM. Poly(hydroxyalkanoate) block or random copolymers of $\beta$-butyrolactone and benzyl $\beta$-malolactone: a matter of catalytic tuning. Macromolecules. 2013;46:6765-76.

[210] Haba O, Tomizuka H, Endo T. Anionic Ring-Opening Polymerization of Methyl 4,6-OBenzylidene-2,3-O- carbonyl-a-D-glucopyranoside : a first example of anionic ring-opening polymerization of five-membered cyclic carbonate without elimination of $\mathrm{CO} 2$. Macromolecules. 2005;38:3562-3.

[211] Azechi M, Matsumoto K, Endo T. Anionic ring-opening polymerization of a fivemembered cyclic carbonate having a glucopyranoside structure. J Polym Sci, Part A: Polym Chem. 2013;51:1651-5. 
[212] Schmitt M, Falivene L, Caporaso L, Cavallo L, Chen EYX. High-speed organocatalytic polymerization of a renewable methylene butyrolactone by a phosphazene superbase. Polym Chem. 2014;5:3261-70.

[213] Coulembier O, De Winter J, Josse T, Mespouille L, Gerbaux P, Dubois P. One-step synthesis of polylactide macrocycles from sparteine-initiated ROP. Polym Chem. 2013;5:21038.

[214] Malik P, Chakraborty D. Hydrogen phosphates: self initiated organocatalysts for the controlled ring-opening polymerization of cyclic esters. Inorg Chim Acta. 2013;400:32-41.

[215] Qiao Y, Yang C, Coady DJ, Ong ZY, Hedrick JL, Yang Y-Y. Highly dynamic biodegradable micelles capable of lysing Gram-positive and Gram-negative bacterial membrane. Biomaterials. 2012;33:1146-53.

[216] Ding X, Yang C, Lim TP, Hsu LY, Engler AC, Hedrick JL, Yang Y-Y. Antibacterial and antifouling catheter coatings using surface grafted PEG-b-cationic polycarbonate diblock copolymers. Biomaterials. 2012;33:6593-603.

[217] Chan JMW, Ke X, Sardon H, Engler AC, Yang YY, Hedrick JL. Chemically modifiable Nheterocycle-functionalized polycarbonates as a platform for diverse smart biomimetic nanomaterials. Chem Sci. 2014;5:3294-300.

[218] Lee ALZ, Venkataraman S, Sirat SBM, Gao S, Hedrick JL, Yang YY. The use of cholesterol-containing biodegradable block copolymers to exploit hydrophobic interactions for the delivery of anticancer drugs. Biomaterials. 2012;33:1921-8.

[219] Zou J, Zhang F, Zhang S, Pollack SF, Elsabahy M, Fan J, Wooley KL. Poly(ethylene oxide)-block-polyphosphoester-graft-paclitaxel conjugates with acid-labile linkages as a $\mathrm{pH}$ sensitive and functional nanoscopic platform for paclitaxel delivery. Adv Healthcare Mater. 2013;3:441-8.

[220] Zhang S, Zou J, Elsabahy M, Karwa A, Li A, Moore DA, Dorshow RB, Wooley KL. Poly(ethylene oxide)-block-polyphosphester-based paclitaxel conjugates as a platform for ultra-high paclitaxel-loaded multifunctional nanoparticles. Chemical Science. 2013;4:2122-6. [221] White JR, Turnbull A. Weathering of polymers: mechanisms of degradation and stabilization, testing strategies and modelling. J Mater Sci. 1994;29:584-613.

[222] Nachtergael A, Coulembier O, Dubois P, Helvenstein M, Duez P, Blankert B, Mespouille L. Organocatalysis paradigm revisited: are metal-free catalysts really harmless? Biomacromolecules. 2014;16:507-14.

[223] Wang Y, Watson MD. Transition-metal-free synthesis of alternating thiopheneperfluoroarene copolymers. J Am Chem Soc. 2006;128:2536-7.

[224] Sanji T, Motoshige A, Komiyama H, Kakinuma J, Ushikubo R, Watanabe S, lyoda T. Transition-metal-free controlled polymerization for poly(p-aryleneethynylene)s. Chem Sci. 2015;6:492-6.

[225] Ogawa KA, Goetz AE, Boydston AJ. Metal-free ring-opening metathesis polymerization. J Am Chem Soc. 2015;137:1400-3.

[226] Treat NJ, Sprafke H, Kramer JW, Clark PG, Barton BE, Read de Alaniz J, Fors BP, Hawker CJ. Metal-free atom transfer radical polymerization. J Am Chem Soc. 2014;136:16096101.

[227] Pan X, Lamson M, Yan J, Matyjaszewski K. Photoinduced metal-free atom transfer radical polymerization of acrylonitrile. ACS Macro Letters. 2015;4:192-6. 